\title{
A network approach to response inhibition and aggression : combining functional imaging and non- invasive brain stimulation in the study of impulse control
}

Citation for published version (APA):

Dambacher, F. (2015). A network approach to response inhibition and aggression : combining functional imaging and non-invasive brain stimulation in the study of impulse control. [Doctoral Thesis, Maastricht University]. Datawyse / Universitaire Pers Maastricht. https://doi.org/10.26481/dis.20150622fd

Document status and date:

Published: 01/01/2015

DOI:

10.26481/dis.20150622fd

Document Version:

Publisher's PDF, also known as Version of record

Please check the document version of this publication:

- A submitted manuscript is the version of the article upon submission and before peer-review. There can be important differences between the submitted version and the official published version of record. People interested in the research are advised to contact the author for the final version of the publication, or visit the DOI to the publisher's website.

- The final author version and the galley proof are versions of the publication after peer review.

- The final published version features the final layout of the paper including the volume, issue and page numbers.

Link to publication

\footnotetext{
General rights rights.

- You may freely distribute the URL identifying the publication in the public portal. please follow below link for the End User Agreement:

www.umlib.nl/taverne-license

Take down policy

If you believe that this document breaches copyright please contact us at:

repository@maastrichtuniversity.nl

providing details and we will investigate your claim.
}

Copyright and moral rights for the publications made accessible in the public portal are retained by the authors and/or other copyright owners and it is a condition of accessing publications that users recognise and abide by the legal requirements associated with these

- Users may download and print one copy of any publication from the public portal for the purpose of private study or research.

- You may not further distribute the material or use it for any profit-making activity or commercial gain

If the publication is distributed under the terms of Article 25fa of the Dutch Copyright Act, indicated by the "Taverne" license above, 


\title{
A network approach to response inhibition and aggression
}

\author{
Combining functional imaging and \\ Non-Invasive Brain Stimulation in the \\ study of impulse control
}

Franziska Dambacher 
ISBN 9789461594488

copyright Franziska Dambacher, Maastricht, The Netherlands, 2015

cover design Rik Wagemans

paranymphs Catharina J. Burgman \& Helen C. Lückmann

printing Datawyse | Universitaire Pers Maastricht

All rights reserved. No part of this publication may be reproduced, stored or transmitted in any form or by any means, without written permission of the copyright owner. 


\title{
A network approach to response inhibition and aggression
}

\author{
Combining functional imaging and \\ Non-Invasive Brain Stimulation in the \\ study of impulse control
}

\author{
DISSERTATION \\ to obtain the degree of Doctor at Maastricht University, \\ on the authority of Rector Magnificus, Prof. Dr. L.L.G. Soete, \\ in accordance with the decision of the Board of Deans, \\ to be defended in public on the $22^{\text {nd }}$ of June 2015 at 14:00 hours
}

by

Franziska Dambacher

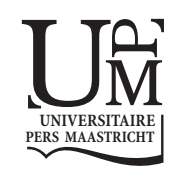




\section{Supervisors}

Prof. Dr. Alexander T. Sack

Prof. Dr. Arnoud Arntz

\section{Co-supervisors}

Dr. Teresa Schuhmann

Dr. Jill Lobbestael

\section{Assessment Committee}

Prof. Dr. Bernadette Jansma (Chair)

Prof. Dr. Chris Chambers (Cardiff University)

Prof. Dr. Ulrike Krämer (Universität zu Lübeck)

Dr. Carolien Martijn

Dr. Vincent van de Ven 
Table of Contents

CHAPTER 1 GENERAL INTRODUCTION

CHAPTER 2 A NETWORK APPROACH TO RESPONSE INHIBITION

DISSOCIATING FUNCTIONAL CONNECTIVITY OF NEURAL

COMPONENTS INVOLVED IN ACTION RESTRAINT AND ACTION

CANCELLATION

CHAPTER 3 THE ROLE OF RIGHT PREFRONTAL AND MEDIAL CORTEX IN RESPONSE INHIBITION

INTERFERING WITH ACTION RESTRAINT AND ACTION CANCELLATION

USING TRANSCRANIAL MAGNETIC BRAIN STIMULATION

CHAPTER 4 OUT OF CONTROL

EVIDENCE FOR ANTERIOR INSULA INVOLVEMENT IN MOTOR IMPULSIVITY AND REACTIVE AGGRESSION

CHAPTER 5 NO EFFECTS OF BILATERAL TDCS OVER INFERIOR FRONTAL GYRUS ON RESPONSE INHIBITION AND AGGRESSION

CHAPTER 6 THE ROLE OF THE INSULAR CORTEX IN RETALIATION

CHAPTER 7 REDUCING PROACTIVE AGGRESSION THROUGH NON-INVASIVE BRAIN STIMULATION

$\begin{array}{lll}\text { CHAPTER } 8 \text { GENERAL DISCUSSION } & 153\end{array}$

$\begin{array}{lr}\text { SUMMARY } & 169\end{array}$

$\begin{array}{ll}\text { VALORIZATION } & 173\end{array}$

$\begin{array}{lr}\text { ACKNOWLEDGEMENTS } & 179\end{array}$

$\begin{array}{lr}\text { CURRICULUM VITAE } & 187\end{array}$ 



\section{CHAPTER 1 General introduction}

"We study the problem and we've been studying it for damn well near a century, yes, but we get no further with our studies. You've got a good home here, good loving parents. You've got not too bad of a brain. Is it some devil that crawls inside of you?"

- Anthony Burgess, A Clockwork Orange, 1962 



\section{THE OMITTED CHAPTER}

In 1962, the British writer Anthony Burgess invented a novel, which attracted vast attention upon its publication. Modern Library included it in the list of the 100 best 20th century Novels written in English. Cineastes admire Burgess' story as it is the source of an all-time favorite movie directed by Stanley Kubrick in 1971 through which it shaped pop culture and iconography of the 70ties: A Clockwork Orange.

The narrative is centered on a young man named Alex and his gang, the 'droogs'. Highly aggressive and impulsive as they are, they find the ultimate pleasure in seemingly arbitrary acts of what they call 'ultra-violence'. Their endeavors that are described in the first part of the book comprise drug abuse, theft, assault, rape, demolition, and child molestation, leaving a trace of destruction wherever they go. When finally arrested, in the second part of the novel Alex is subjected to the 'Ludovico Technique', an aversive procedure of behaviour-modification based on the principles of classical conditioning; while injected with a substance that induces severe nausea, he is forced to watch videos graphically depicting all kinds of cruelty. This leads to an immediate coupling of violence and nausea, which is supposed to erase all aggressive tendencies from his personality. The third part of the book is devoted to Alex's life after prison (Burgess, 1986).

When Burgess proposed his manuscript to an American publisher, he was strongly advised that the 21st and final chapter of the work should be omitted in the oversea's version. Indeed, editions published in the United States prior to 1986 consist of only 20 instead of 21 chapters and so does Kubrick's movie. In the original ending proposed by the author, Alex finally starts to reflect on his actions and the crimes he committed. He gains deeper insights in his past life, personality, and the ethical values of society and ultimately renounces violence and pledges to change his behaviour for the better (Faizan, 2012).

Apparently, the original ending did not appear catchpenny enough to publishers and producers; in the movie as well as the shortened version of the novel, Alex is never freed of his aggressive tendencies and the story ends dark and crestfallen. As literary circles have debated for half a century which fits Burges' novel best, scientists have wondered about whether or not impulsivity and aggression can be modulated on a behavioural or biological level. The doctoral thesis at hand, tries to contribute empirical evidence to this discussion.

Impulsivity and aggression have always fascinated human kind. References range from ancient drawings and religious stories to modern day literature, cinema, and television. In the Christian mythology, the second event that happens right after earth and life are created is a murder; Kain, the first son of Adam and Eve, kills his younger brother Abel (Genesis 4:1-8, Holman Christian Standard Bible). Following this myth, already the second human born did not die from natural causes but by human hand. Parallels can be found in all cultures and religions. 


\section{CHAPTER 1}

Upon this day crime seems the most popular and best-selling feature in the media landscape. The British Guardian declared that almost two-thirds of all books borrowed from British libraries in 2013 were crime novels (British library charts 2013).

Probably more impacting than the cultural perception of impulsivity and aggression is its societal relevance. Every year more than 180.000 registered 'violent crimes' (including murder, manslaughter, rape, sexual coercion, robberies, dangerous and serious bodily injury, and slight bodily injury with intent) are registered by German authorities (Polizeiliche Kriminalstatistik PKS; Bundeskriminalamt BKA, 2013). In the United States, violent crimes amount to over one million per year (Uniform Crime Report UCR; Federal Bureau of Investigation FBI, 2013). The prevalence of aggressive behaviour is one of the main challenges our modern civilization has to face. It leads to the experience of insecurity in all of us and poses enormous costs to our social systems. Therefore, it is crucial to provide the public, forensic professionals, and workers dealing with aggressive individuals (in schools, hospitals, and the social sector) with scientific evidence on 1) etiological models and 2) potential interventions to decrease aggression.

\section{SOCIAL NEUROSCIENCE}

Cacioppo and colleagues (2000) have repeatedly pointed out how crucial it is to analyze human behaviour on multiple levels in order to gain comprehensive knowledge. The neural correlates of social interaction attract growing interest in neuroscience. Although seemingly complex and difficult to pin down in a laboratory situation, research involving neuroscientific methodology starts to identify neural processes underlying social constructs. Complementary social and biological perspectives need to be integrated into what we nowadays call 'social neuroscience'.

We as humans are 'social animals' (Aronson, 1972); our behaviour cannot be evaluated independently from our social environment. Most actions that we commit on a daily basis are closely linked to our surroundings. Social and cultural factors do not only influence behaviour, but also the biological processes underlying it. This has been shown in the context of genetics, immunology, cardiovascular functioning, and pathology (Cacioppo et al., 2000). For instance, Reynolds and Berridge (2008) have observed that varying emotional environments retunes the function of neural populations. On another note, the field of epigenetics has demonstrated, that the social and emotional environment an individual is exposed to can tremendously change how and which biological components underlying behaviour (in this case genes) are expressed (Bird, 2007). The link between biology, social interactions, and the brain should be considered in a multilevel approach to understanding human behaviour. 


\section{FROM LESION STUDIES TO NON-INVASIVE METHODOLOGY IN NEUROSCIENCE}

In order to investigate the link between behaviour and the brain, various neuroscientific methodologies are at disposal. In history, knowledge gain on the brain relied on lesion studies. One of the first official - and definitely the most famous - cases investigated in 'social neuroscience' was that of Phineas Gage (Damasio, Grabowski, Frank, Galaburda, \& Damasio, 1994). No textbook on psychology or basic neuroscience is complete without his story and pictures. After his prefrontal cortex was severely lesioned due to an accident in 1884, he showed alterations in his cognitive control, became impulsive, and was no longer capable of goal-oriented behaviour which ultimately led to severe changes in his social behaviour. More recent case studies also demonstrate a link between frontal lobe lesions and impulsivity, violence, and aggression. It was shown that Vietnam veterans suffering from frontal lobe lesions display more aggressive and violent behaviour (Grafman et al., 1996) than their comrades. In patients with early onset frontal lobe damage, impaired social and moral judgement was observed; although not diagnosed with psychopathic traits, these patients showed behaviours that are typical for psychopaths (Anderson, Bechara, Damasio, Tranel, \& Damasio, 1999).

Lesions sometimes have to be surgically created to improve patients' symptoms. This was done e.g. in the context of treatment-resistant epilepsy (Ameli, 1980; Morris, 1956). Recently, a verdict of a Brazilian court suggested an active amygdala lesion in three mentally handicapped adults with impulsivity issues (Gouveia et al., 2014). This surgical intervention was executed despite the severe side effects which were to be expected. In certain circumstances, such intervention can be ethically questionable.

Lesion studies provide important information on how the brain works. They nevertheless have several disadvantages (Sternberg, 1999): First, patients with specific brain lesions are rare and cannot be investigated with randomized and well-controlled experimental designs. Therefore, results cannot be generalized. Second, lesions are often not well defined and not bound to specific brain regions. The lesioned brain initiates processes in order to compensate for the damage. These processes induce additional variance into patient groups with similar lesions and make it difficult to trace behavioural consequences. Third, functional deficits in patients with brain lesions do not necessarily coincide well with the location of their lesion. Finally, the induction of brain lesions as in the above mentioned cases can have severe side effects. Due to the described disadvantages, information derived by investigating the lesioned brain cannot be sufficient to gain insights on the function of the intact brain. Evidence derived from different methodologies has to be integrated in order to reveal neural mechanisms and their relation to behaviour on multiple levels. In the field of cognitive neuroscience, non-invasive methods provide promising tools to circumvent the described problems. The research presented in this thesis made use of non-invasive methods enabling 
the localization (Brain Imaging) and the manipulation (Non-Invasive Brain Stimulation) of brain function.

\section{Brain Imaging}

Cognitive neuroscience relies vastly on methods, which allow the localization of anatomical structures as well as functional activation within these structures. For instance, $\mathrm{X}$-Ray and Positron Emission Tomography fulfill this purpose. Unlike these methods, Magnetic Resonance Imaging (MRI) is based solely on tissue properties and metabolism and does not require exposure to any ionizing radiation or contrast medium. Thus, it is a powerful and non-invasive tool in the context of brain imaging.

Magnetic Resonance Imaging (MRI). By exposing tissue to a strong static magnetic field, protons in hydrogen atoms within the tissue align themselves to this external magnetic field. When rapid alternating radiofrequency pulses are applied, these protons oscillate and deviate from their original alignment. The local energy fields that are emitted as the protons return back to their original alignment depend on specific tissue properties. By recording this signal, tissue properties can be reconstructed. Next to spinal imaging, brain imaging is currently the most prevalent use of MRI ("Magnetic Resonance, a critical peer-reviewed introduction", European Magnetic Resonance Forum, retrieved January, $\left.14^{\text {th }}, 2015\right)$. In the context of neuroscience, this enables the mapping of brain structure and anatomy, the imaging of specific anatomical features, and the detection of deviant, e.g. cancerous, tissue.

Functional Magnetic Resonance Imaging (fMRI). One of the most crucial methodological advances in cognitive neuroscience was the development of tools that can link specific anatomical structures to certain cognitive processes (Raichle, 1994). FMRI undoubtedly contributed immensely to this development. When a brain region is activated during cognitive or behavioural processes, it has to be supplied with oxygen via the blood supply. A time-locked measurement of oxygenated blood within brain regions during specific functional requirements (during e.g. task performance) can, therefore, shed light on the functional involvement of these brain regions. FMRI exploits exactly this blood oxygenation level-dependent (BOLD) signal in order to reveal the function of brain regions and the spatial distribution of their functional involvement. FMRI has a rather low temporal resolution compared to other neuroscientific methods such as, for instance, Electroencephalography. Its spatial resolution, however, is higher than in most other non-invasive imaging methods; this holds true especially for high-field fMRI.

FMRI can provide information on which brain regions are involved in a cognitive process and where they are located (standard approach). In addition, the method also allows to investigate if (functional connectivity) and how (directed connectivity) brain areas interact during a certain cognitive process (Goebel, Roebroeck, Kim, \& 
Formisano, 2003; Roebroeck, Formisano, \& Goebel, 2005). Both approaches have been applied in the context of this thesis.

While $\mathrm{fMRI}$ is a powerful tool to reveal associations between brain structure and function, it cannot shed light on the causality of these associations and, furthermore, cannot interfere with brain activity. Therefore, the combination of fMRI with NonInvasive Brain Stimulation techniques can lead to further knowledge gain on the causal relationship between brain activation and impulsivity and aggression.

\section{Non-Invasive Brain Stimulation}

Non-Invasive Brain Stimulation techniques allow manipulating brain activity, i.e. increasing or decreasing the activity or excitability of a specific brain region. This enables three-fold knowledge-gain: First, manipulating the activity of specific brain regions and subsequently observing behavioural consequences leads to a better understanding of the functional role of these regions in the investigated behaviour. Second, this approach permits more causal or mechanistic interpretations of brain-behaviour relationships than evidence obtained by means of mainly correlational methodology like fMRI (Sack, 2006). Finally, Non-Invasive Brain Stimulation - in its ability to manipulate - is a method which can change brain activity patterns and, thus, can potentially be translated to clinical applications (Fregni, Freedman, \& Pascual-Leone, 2007; Hummel \& Cohen, 2006; Williams, Imamura, \& Fregni, 2009). In the following, the two NonInvasive Brain Stimulation techniques employed in the research presented in this thesis will be described in detail.

Transcranial Magnetic Brain Stimulation (TMS). TMS interferes with brain activity exploiting the law of electromagnetic induction. Standard TMS equipment consists of a stimulator and a connected figure-of-eight coil. By holding it to the scalp surface and generating a powerful electrical field within the coil, a magnetic field (perpendicular to the electric field) is induced, which in turn induces another electric field (perpendicular to the magnetic field) within neural tissue. Depending on the TMS-protocol administered, neurons - being electro-conductive elements within the brain - react to this induction of electric current (Hallett, 2000).

Protocols. In terms of pulse patterns in which TMS can be administered, mainly two types of protocols can be differentiated. One can employ single pulse TMS at various time points within a given cognitive process in order to investigate, for instance, the chronological aspects of functional involvement regarding certain brain regions. For patterned TMS (Rossi, Hallett, Rossini, \& Pascual-Leone, 2009) multiple TMS pulses are administered in trains. It serves to induce reversible but relatively long-lasting alterations of brain activity and enables the observation of changes in behaviour due to these changes in neural activity. This procedure is also named the 'virtual lesion approach' (Pascual-Leone, Bartres-Faz, \& Keenan, 1999). While low frequency (1 Hz or less) repetitive TMS decreases, while high frequency (more than $1 \mathrm{~Hz}$ ) repetitive TMS 
increases activity in the stimulated brain region (Rossi et al., 2009). The after-effects of repetitive TMS last about as long as stimulation is administered. Thetaburst Stimulation (TBS; Huang, Edwards, Rounis, Bhatia, \& Rothwell, 2005) is another form of patterned TMS which causes relatively long lasting effects with short stimulation periods: Continuous TBS ( 3 pulses of stimulation at $50 \mathrm{~Hz}$, repeated every $200 \mathrm{~ms}$, for 40s, resulting in 600 pulses) inhibits brain activity for up to 30 to 40 minutes, whereas intermittent TBS ( $3 \mathrm{~s}$ trains of TBS, repeated every $10 \mathrm{~s}$, for a total of $190 \mathrm{~s}$, resulting in 600 pulses) has excitatory effects. Intermediate TBS ( $5 \mathrm{~s}$ trains of TBS, repeated every $15 \mathrm{~s}$, for a total of $110 \mathrm{~s}$, resulting in 600 pulses) shows neither inhibitory nor facilitatory effects.

Localization of target regions. In order to localize potential TMS target regions and subsequently position the TMS coil accordingly, mainly three approaches have been used (Sack et al., 2009). First, regions can be targeted by placing the TMS coil over associated positions within the international 10-20- or respectively 10-10 EEG system (Oostenveld \& Praamstra, 2001) on the scalp. While not posing any methodological or materialistic challenges and being cheap, easy, and fast, this approach takes interindividual variability in neither brain structure nor brain function into account. Second, in order to account for anatomical variability in brain structure, localization procedures for TMS can be based on individual anatomical MRI scans. Therefore, structural brain scans of each participant have to be acquired which are then, together with the TMS coil and the head surface of the participant, aligned in space by means of a stereotactic system. This ensures that TMS pulses are delivered precisely over the anatomical landmarks that are to be targeted in each individual. Third, in order to account for inter-individual variability not only in anatomy, but also in spatial distribution of function, the positioning of the TMS coil over individual target sites can be based on individual functional imaging data relevant to the concept at investigation. In this case we speak of 'fMRI guided' TMS. It has been demonstrated that individuals differ significantly in the spatial distribution of functional brain activity (Frost \& Goebel, 2012). Therefore, 'functionally guided' TMS increases the statistical power of TMS experiments by accounting for this variability (Sack et al., 2009; Sparing, Hesse, \& Fink, 2010). In the context of this thesis fMRI guided continuous TBS was applied.

Safety issues. TMS is a non-invasive method of brain stimulation, which allows to better understand timing and causality with respect to functional involvement of brain regions. To apply TMS pain-free and with minimal risk, certain safety guidelines have to be met. There is a low risk to induce a seizure (for high frequency repetitive TMS: $1.4 \%$ in epileptic patients, under $1 \%$ in healthy individuals). TMS can be accompanied by transient head ache, local pain, neck pain, toothache, paresthesia, and transient changes in hearing (Rossi et al., 2009). To ensure the safe application of TMS, only TMS protocols within the current safety guidelines can be employed (Rossi et al., 2009; Wassermann, 1998), proper hearing protection has to be used, and every participant needs to be thoroughly screened to rule out any potential risk factors (e.g. individual / 
family history of epilepsy, psychiatric or neurological disorders, drug (ab)use, sleep deprivation).

Transcranial Current Stimulation (tCS). Another form of Non-Invasive Brain Stimulation is tCS (Nitsche et al., 2008; Paulus, 2011). TCS induces low electric currents within brain tissue and, thereby, increases or decreases the excitability of brain regions depending on the polarity of current induced. A standard tCS setup consists of a battery to which two electrodes (which can vary in size) are connected. By positioning those electrodes on the scalp (for procedure see DaSilva, Volz, Bikson, \& Fregni, 2011) using a conductive medium (saline solution or electroconductive gel), current can be generated within brain tissue. The current flow is always directed from the anodal electrode (positively polarized) towards the cathodal electrode (negatively polarized). The latter electrode is often also called the 'reference' electrode. The excitability of brain areas that are hyperpolarized (by anodal stimulation) is increased, while the excitability of brain areas that are hypopolarized (by cathodal stimulation) is decreased. This effect is caused by a manipulation of the membrane potential of neurons leading to alterations in spontaneous firing rates (Paulus, 2011). How current flow, current density, and current polarity are distributed within brain tissue depends on the exact montage of anode and cathode on the scalp (Nitsche et al., 2007).

Protocols. TCS can be applied in various forms. Transcranial Direct Current Stimulation (tDCS) induces stable currents of fixed intensity (usually about 0.5 to $3.0 \mathrm{~mA}$ ) over a period of up to 40 minutes; excitatory effects are demonstrated for anodal, while inhibitory effects have been shown for cathodal stimulation (Nitsche et al., 2008). Transcranial Alternating Current Stimulation (tACS; Antal \& Paulus, 2013) is a method capable to influence brain oscillations. This is achieved by applying currents in a time dependent manner following a sinusoidal function. Thereby, the amplitude, frequency, and relative phases of brain oscillations in areas across stimulation electrodes can be varied. TACS is a powerful tool that can either synchronize or desynchronize the coupling of oscillations within brain regions. For transcranial Random Noise Stimulation (tRNS; Terney, Chaieb, Moliadze, Antal, \& Paulus, 2008) currents are applied randomly over a certain amount of time; the technique is relatively new and, thus, experience with it is limited, but the main effects of tRNS seem to be excitatory (Terney et al., 2008).

Localization of target regions. TCS can stimulate brain regions selectively. However, the degree of precision with which specific brain regions can be targeted falls below that of TMS. Attempts have been made to develop adequate procedures to correctly model current location and density within potential target regions (Datta, Baker, Bikson, \& Fridriksson, 2011; Jung, Kim, \& Im, 2013; Neuling, Wagner, Wolters, Zaehle, \& Herrmann, 2012; Oostendorp et al., 2008; Wagner et al., 2007). Although such modelling approaches are not yet common practice in tCS research, it will be crucial to take into account actual tissue properties and inter-individual differences in brain anatomy, when optimizing electrode positioning in order to best reach potential target sites in 
the future (Dannhauer, Brooks, Tucker, \& MacLeod, 2012; Rampersad, Stegeman, \& Oostendorp, 2011).

Safety issues. TCS is described as risk-free. Few minor aversive side effects related to the method have been reported in healthy participants and patients (Poreisz, Boros, Antal, \& Paulus, 2007). Mostly, participants in tCS studies report a tingling sensation at the beginning and the end of the stimulation period (reported by around $70 \%$ of participants), moderate fatigue (around 35\%), and sometimes an itching sensation at the position of the electrode (around 30\%; Poreisz et al., 2007). Rarely, participants report headache (around 11\%), nausea (around 25\%), or insomnia (around $0.95 \%$ ) after tCS stimulation (Poreisz et al., 2007). So far, no severe side effects of tCS are reported in the literature. The induction of electric current can cause biochemical reactions on the skin surface and, therefore, only participants who do not suffer from severe skin diseases should undergo the method and current safety standards regarding type and intensity of induced current and exposure time to stimulation should not be exceeded in order to avoid skin injuries (Bikson, Datta, \& Elwassif, 2009).

TMS versus tCS. It has to be emphasized that TMS and tCS - although both being non-invasive brain inference methods - are different in nature. Both bring along certain advantages and disadvantages, which make them more or less suitable for specific research questions and practical contexts. TMS is a powerful and well-established method that allows for precise stimulation of specific brain regions. Especially in its combination with $\mathrm{fMRI}$ and by targeting brain regions based on individual functional localization it can lead to a level of causal insight regarding functional involvement of brain structures which is unparalleled in humans. The physiological mechanisms underlying tCS, on the other hand, are not yet well established (Horvath, Forte, \& Carter, 2014). Furthermore, spatial specificity of tCS is relatively low (as compared to TMS) and localization procedures for tCS target regions and their optimization are currently developed (Dannhauer et al., 2012; Neuling et al., 2012; Oostendorp et al., 2008; Rampersad et al., 2011). However, tCS also has advantages over TMS. Being a cheap, highly portable, and mainly risk-free neuroscientific tool, it is interesting as a method for potential clinical interventions. Furthermore, it provides a way to test relatively global hypotheses on either relatively large brain structures (e.g. the prefrontal cortex) or cortical balance theories, where directed hypotheses on which specific brain regions should be target of interventions are not available. This makes tCS a useful method for the investigation of complex or higher order behaviour, as it is essential in social neuroscience. Finally, in the scientific context, tCS has one undeniable advantage over TMS; it provides a control, sham, or placebo condition that is easy to implement and cannot be differentiated from real stimulation (Paulus, 2011). While sham TMS applications mainly manage to imitate the auditory stimulus related to TMS pulses, but not the skin sensations related to real stimulation, sham tCS is not even recognizable for experienced participants. In order to apply sham tCS the electrodes are positioned equal to real stimulation, but the stimulation is switched of immediately after the 
ramping phases. This procedure mimics the skin sensation accompanying tCS applications and, thus, reliably deceives participants about which condition they are assigned to.

\section{RESPONSE INHIBITION AND ITS RELATION TO AGGRESSION}

In this thesis the described neuroscientific methods were used as tools to investigate two basic concepts and how they relate to each other on neural level: Response inhibition and aggression. Both, in turn, are closely linked to impulsivity.

Logan and colleagues (1997) emphasize the importance of impulsivity as a concept in psychology: It plays a prominent role in differential psychology and is part of the extraversion dimension in many personality theories (Eysenck \& Eysenck, 1969; Revelle, Humphreys, Simon, \& Gilliland, 1980). In developmental psychology, impulsivity is a crucial component of various disorders such as attention deficit hyperactivity and conduct disorder (Quay, 1998). However, 'dis-inhibitory psychopathologies' (Gorenstein \& Newman, 1980) are by far not restricted to childhood; impulsivity is also a crucial feature in obsessive compulsive disorder, certain expressions of schizophrenia, borderline, and antisocial personality disorder.

Aside from its clinical relevance, impulsivity is a concept important to everyday life: Individuals are often characterized by their actions. However, it might be that the things we chose not to do rather than the things we do that make us who we are. In every moment in which a human being choses one action, simultaneously countless other behavioural options have to be inhibited. It has been shown that people who do best in inhibiting alternative actions - and thus act goal-oriented - respect social norms, are not dependent on immediate gratification, and generally do best in life (Eigsti et al., 2006; Mischel, Shoda, \& Peake, 1988; Mischel, Shoda, \& Rodriguez, 1989). On brain level this is mirrored by the fact that amongst all mammals, the human species is equipped with the functionally most developed prefrontal cortex - a region repeatedly associated with cognitive control (Koechlin, Ody, \& Kouneiher, 2003; Miller, 2000).

One way to measure impulsivity on a behavioural level is to examine the ability to inhibit motor responses. Response or action inhibition is defined as the withholding of pre-potent courses of action (Logan et al., 1997). Different forms of inhibition can be differentiated (Eagle, Bari, \& Robbins, 2008): Action restraint refers to the inhibition of an action tendency which has not yet been initiated; it can be measured with classic go/no-go paradigms in which participants have to respond to a frequent go stimulus, while they have to restrain their response to an infrequent no-go stimulus. Action cancellation, on the other hand, refers to the inhibition of an action which has already been initiated; this can be measured with stop signal paradigms, in which participants have to respond to a go stimulus and cancel this response whenever they are presented with a stop signal shortly after the go stimulus. 


\section{CHAPTER 1}

Imaging studies on various response inhibition paradigms consistently reported task-related activity within inferior frontal gyrus, pre-supplementary motor area, anterior insula, and subcortical circuitries including thalamus and striatum (Chambers, Garavan, \& Bellgrove, 2009; Simmonds, Pekar, \& Mostofsky, 2008; Swick, Ashley, \& Turken, 2011). Response inhibition related brain activity was shown to be largely rightlateralized (Aron, Robbins, \& Poldrack, 2004).

Aggression - defined as behaviour that aims to intentionally harm others on psychological or physical levels (Anderson \& Bushman, 2002) - has empirically been associated with impaired executive functioning and inhibition (Hoaken, Shaughnessy, \& Pihl, 2003). Looking at how response inhibition related neural activity was mirrored in trait impulsiveness and self-reported aggression led to important insights. Horn and colleagues (2003) showed that impulsive individuals relied on stronger right orbitofrontal activity to maintain inhibitory capacities in a go/no-go task. Furthermore, response inhibition related activity in the right dorsolateral prefrontal cortex was found to be negatively correlated with motor impulsiveness measured by the Barratt Impulsiveness Scale (Asahi, Okamoto, Okada, Yamawaki, \& Yokota, 2004). Recently, Pawliczek and colleagues (2013) showed that high trait aggressiveness is associated with inhibition deficits and lower inhibition related brain activity.

As the term aggression comprises rather complex aspects of behaviour, it is usually categorized into sub-types, e.g. depending on different motivational states underlying it; while proactive aggression refers to using aggression in an instrumental, goaloriented way, reactive aggression refers to aggressive actions in response to preceding provocation (Poulin \& Boivin, 2000; Raine et al., 2006). The described literature does not differentiate between these types of aggression, although the neural correlates as well as the deficits in executive functioning associated with re- versus proactive aggression might differ. Furthermore, the described neuroscientific evidence relied on mainly self-reported measures of impulsivity and aggression and omitted behavioural aggression measures. However, it is a long- and well-known problem that behavioural measures of social constructs do not necessarily overlap with measures on a selfreported level (Scheier, Buss, \& Buss, 1978). Especially in aggression research, effects of social desirability and lack of self-insight are obstacles that measurement tools have to overcome (Vigil-Colet, Ruiz-Pamies, Anguiano-Carrasco, \& Lorenzo-Seva, 2012). Although well-controlled behavioural aggression paradigms such as the Taylor Aggression Paradigm (Krämer, Jansma, Tempelmann, \& Münte, 2007; Taylor, 1967) exist they have not often been used in neuroscience. The current challenge of research in social neuroscience is to look into behavioural measures of impulsivity and aggression, investigate the underlying neural correlates, and link these to established neural networks and theories on cognitive control. The work presented in this thesis followed this approach.

Research on the relationship between measures of impulsivity and aggression and its neural correlates does not only advance scientific knowledge about aggression and 
its underlying mechanisms but ultimately lets science contribute to solve actual societal problems. A better understanding of aggression can help to better deal with violence in our societies and to develop interventions on behavioural and brain level that are able to decrease aggression. Neither as scientists nor as citizens we should 'omit' the crucial questions: Can aggressive behaviour be changed and, if so, how can violent individuals be re-integrated in our community? From this perspective, the so often omitted $21^{\text {st }}$ chapter from Burges' novel 'A Clockwork Orange' is actually the most interesting one for the $21^{\text {st }}$ century.

\section{OUTLINE OF THE THESIS}

The thesis at hand is divided into three parts: Chapter 1 and 2 are focused on the investigation of response inhibition networks; in chapter 3 and 4 the overlap of neural correlates between motor impulsivity and aggression is discussed; chapter 6 and 7 zoom into the neural components underlying different forms of aggression. Every part consists of $\mathrm{fMRI}$ work (revealing the neural correlates of the investigated concepts and their localization) and work applying Non-Invasive Brain Stimulation techniques (manipulating these neural correlates and observing subsequent changes in behaviour). In the following, the content of the single chapters is outlined:

In a first step, we aimed to reveal the neural correlates of one form of executive control related to aggression, namely motor response inhibition. We hypothesized that different aspects of response inhibition would translate to different neural activation patterns, but also activate a global common inhibition network within the brain. The fMRI study "A network approach to response inhibition: Dissociating functional connectivity of neural components involved in action restraint and action cancellation" (chapter 2) was designed to test this hypothesis by revealing the neural correlates of action restraint and action cancellation. Subsequently, we targeted the common inhibition network as well as the specific neural components revealed in the first study with cTBS ("The role of right prefrontal and medial cortex in response inhibition: Interfering with action restraint and action cancellation using transcranial magnetic brain stimulation", chapter 3) to find out, whether a) we could establish a mechanistic or causal link between the identified brain regions and the investigated aspects of response inhibition and $b$ ) if the capacity to inhibit pre-planned or automatic responses could be modified by means of Non-Invasive Brain Stimulation.

In a second step, we investigated whether the neural correlates we identified to be involved in response inhibition overlap with neural networks involved in aggression. As outlined above, research has shown that failed response inhibition (or motor impulsivity) is related to aggression on behavioural level. We hypothesized that both concepts should also be related on brain level and that their neural correlates should (at least in part) overlap. Chapter 4 ("Out of control: Evidence for anterior insula involve- 
ment in motor impulsivity and reactive aggression") focuses on the investigation of this overlap between brain regions involved in motor impulsivity and behavioural aggression employing fMRI during a response inhibition and a controlled behavioural aggression paradigm. Subsequently, we manipulated the excitability of one overlapping region - namely the inferior frontal cortex - by means of bilateral tDCS ("No effects of bilateral tDCS over inferior frontal gyrus on response inhibition and aggression", chapter 5) investigate whether motor response inhibition and / or aggressive behaviour could be altered by this intervention. It was expected that enhancing the right inferior frontal gyrus (while simultaneously inhibiting the left) should increase the ability to inhibit motor responses and at the same time decrease aggressive behaviour, whereas enhancing the left inferior frontal gyrus (while simultaneously inhibiting the right) should have the exact opposite effect.

In a final step, we focused on the neural correlates of aggressive behaviour. In chapter 6, entitled "Left posterior insular activation during retaliation", parametric modulations of brain activity related to aggressive behaviour were analyzed in order to further investigate the role of the insular cortex in aggression. This region was repeatedly reported to be involved in aggression and we tested our hypothesis that based on previous literature regarding insular function, specifically the posterior part should be involved in retaliation (aggression in reaction to provocation) and that this activation should be left-lateralized. In the concluding study ("Reducing proactive aggression through Non-Invasive Brain Stimulation", chapter 7) we aimed to investigate, if aggressive behaviour can be altered by enhancing the right dorsolateral prefrontal cortex with tDCS. Thereby, it was expected that an enhancement of this region should cause an increase in avoidance motivation and, thus, decrease aggression.

The results of all empirical studies will be summarized and discussed in the final chapter 8 ("General discussion"). Implications for future research, resulting challenges in social neuroscience, and potential clinical applications of the results outlined in this thesis will be illustrated. 
Ameli, N. O. (1980). Hemispherectomy for the treatment of epilepsy and behavior disturbance. The Canadian Journal of Neurological Sciences. Le Journal Canadien Des Sciences Neurologiques, 7, 33-38.

Anderson, C. A., \& Bushman, B. J. (2002). Human aggression. Annual Review of Psychology, 53, 27-51.

Anderson, S. W., Bechara, A., Damasio, H., Tranel, D., \& Damasio, A. R. (1999). Impairment of social and moral behavior related to early damage in human prefrontal cortex. Nature neuroscience, 2(11), 10321037.

Antal, A., \& Paulus, W. (2013). Transcranial alternating current stimulation (tACS). Frontiers in Human Neuroscience, 7(6), 317.

Aron, A. R., Robbins, T. W., \& Poldrack, R. A. (2004). Inhibition and the right inferior frontal cortex. Trends in Cognitive Sciences, 8(4), 170-7.

Aronson, E. (1972). The social animal. New York: Worth Publishers.

Asahi, S., Okamoto, Y., Okada, G., Yamawaki, S., \& Yokota, N. (2004). Negative correlation between right prefrontal activity during response inhibition and impulsiveness: a fMRI study. European Archives of Psychiatry and Clinical Neuroscience, 254(4), 245-51.

Bikson, M., Datta, A., \& Elwassif, M. (2009). Establishing safety limits for transcranial direct current stimulation. Clinical Neurophysiology : Official Journal of the International Federation of Clinical Neurophysiology, 120(6), 1033-4.

Bird, A. (2007). Perceptions of epigenetics. Nature, 447(7143), 396-8.

British library charts, 2013. http://www.theguardian.com/ books/2014/feb/14/most-borrowed-librarybooks-analysis. Retrieved on January 16th, 2014.

Burgess, A. (1986). A Clockwork Orange. New York: Norton.

Cacioppo, J. T., Berntson, G. G., Sheridan, J. F., \& McClintock, M. K. (2000). Multilevel integrative analyses of human behavior: social neuroscience and the complementing nature of social and biological approaches. Psychological Bulletin, 126(6), 829-43.

Chambers, C. D., Garavan, H., \& Bellgrove, M. A. (2009). Insights into the neural basis of response inhibition from cognitive and clinical neuroscience. Neuroscience and Biobehavioral Reviews, 33(5), 631-46.

Damasio, H., Grabowski, T., Frank, R., Galaburda, A. M., \& Damasio, A. R. (1994). The return of Phineas Gage: clues about the brain from the skull of a famous patient. Science, 264, 1102-1105.

Dannhauer, M., Brooks, D., Tucker, D., \& MacLeod, R. (2012). A pipeline for the simulation of transcranial direct current stimulation for realistic human head models using SCIRun/BioMesh3D. Conference Proceedings: Annual International Conference of the IEEE Engineering in Medicine and Biology Society, 5486-9.

DaSilva, A. F., Volz, M. S., Bikson, M., \& Fregni, F. (2011). Electrode positioning and montage in transcranial direct current stimulation. Journal of Visualized Experiments, 51.

Datta, A., Baker, J. M., Bikson, M., \& Fridriksson, J. (2011). Individualized model predicts brain current flow during transcranial direct-current stimulation treatment in responsive stroke patient. Brain Stimulation, 4(3), 169-74.

Eagle, D. M., Bari, A., \& Robbins, T. W. (2008). The neuropsychopharmacology of action inhibition: crossspecies translation of the stop-signal and go/no-go tasks. Psychopharmacology, 199(3), 439-56.

Eigsti, I.-M., Zayas, V., Mischel, W., Shoda, Y., Ayduk, O., Dadlani, M. B., ... Casey, B. J. (2006). Predicting cognitive control from preschool to late adolescence and young adulthood. Psychological Science, 17(6), 478-84.

Eysenck, H.J. \& Eysenck, S.B.G. (1969). Personality Structure and Measurement. San Diego: Knapp.

Faizan, A. (2012). A Clockwork Orange, Chapter 21: The omittance of change. Retrieved from http://www.academia.edu/3817137/ A_Clockwork_Orange_Chapter_21_The_omittance_of_change, 27.12.2014.

Fregni, F., Freedman, S., \& Pascual-Leone, A. (2007). Recent advances in the treatment of chronic pain with Non-Invasive Brain Stimulation techniques. The Lancet, Neurology, 6(2), 188-91.

Frost, M. A., \& Goebel, R. (2012). Measuring structural-functional correspondence: spatial variability of specialised brain regions after macro-anatomical alignment. Neurolmage, 59(2), 1369-81. 


\section{CHAPTER 1}

Goebel, R., Roebroeck, A., Kim, D.-S., \& Formisano, E. (2003). Investigating directed cortical interactions in time-resolved $\mathrm{fMRI}$ data using vector autoregressive modeling and Granger causality mapping. Magnetic Resonance Imaging, 21(10), 1251-61.

Gorenstein, E. E., \& Newman, J. P. (1980). Disinhibitory psychopathology: A new perspective and a model for research. Psychological Review, 87(3), 301-315.

Gouveia, F.V., Oliveira, C.C., Santos, L.T.C., Seno M.D.J., Fonoff, E.T., Alho, E., Rigonatti, S.P., Otoch, J.P., Teixeira, M.J., \& Martinez, C.R. (2015). The role of amygdala in human aggression disorders. Poster presented at the annual meeting of the Society for Neuroscience, 2015, Washington DC.

Grafman, J., Schwab, K., Warden, D., Pridgen, A., Brown, H. R., \& Salazar, A. M. (1996). Frontal lobe injuries, violence, and aggression: a report of the Vietnam Head Injury Study. Neurology, 46, 1231-1238.

Hallett, M. (2000). Transcranial magnetic stimulation and the human brain. Nature, 406(6792), 147-50.

Hoaken, P. N. S., Shaughnessy, V. K., \& Pihl, R. O. (2003). Executive cognitive functioning and aggression: Is it an issue of impulsivity? Aggressive Behavior, 29(1), 15-30.

Horn, N. R., Dolan, M., Elliott, R., Deakin, J. F. W., \& Woodruff, P. W. R. (2003). Response inhibition and impulsivity: an fMRI study. Neuropsychologia, 41(14), 1959-66.

Horvath, J. C., Forte, J. D., \& Carter, O. (2014). Evidence that transcranial direct current stimulation (tDCS) Generates little-to-no reliable neurophysiologic effect beyond MEP amplitude modulation in healthy Human subjects: A systematic review. Neuropsychologia, 66, 213-236.

Huang, Y.-Z., Edwards, M. J., Rounis, E., Bhatia, K. P., \& Rothwell, J. C. (2005). Theta burst stimulation of the human motor cortex. Neuron, 45(2), 201-6.

Hummel, F. C., \& Cohen, L. G. (2006). Non-Invasive Brain Stimulation: a new strategy to improve neurorehabilitation after stroke? The Lancet. Neurology, 5(8), 708-12.

Jung, Y.-J., Kim, J.-H., \& Im, C.-H. (2013). COMETS: A MATLAB toolbox for simulating local electric fields generated by transcranial direct current stimulation (tDCS). Biomedical Engineering Letters, 3(1), 39-46.

Koechlin, E., Ody, C., \& Kouneiher, F. (2003). The architecture of cognitive control in the human prefrontal cortex. Science, 302(5648), 1181-5.

Logan, G. D., Schachar, R. J., \& Tannock, R. (1997). Impulsivity and Inhibitory Control. Psychological Science, $8(1), 60-64$.

Miller, E. K. (2000). The prefrontal cortex and cognitive control. Nature Reviews Neuroscience, 1(1), 59-65.

Mischel, W., Shoda, Y., \& Peake, P. K. (1988). The nature of adolescent competencies predicted by preschool delay of gratification. Journal of Personality and Social Psychology, 54(4), 687-96.

Mischel, W., Shoda, Y., \& Rodriguez, M. I. (1989). Delay of gratification in children. Science, 244(4907), 9338.

Morris, A. A. (1956). Temporal lobectomy with removal of uncus, hippocampus, and amygdala; results for psychomotor epilepsy three to nine years after operation. A. M. A. Archives of Neurology and Psychiatry, 76, 479-496.

Neuling, T., Wagner, S., Wolters, C. H., Zaehle, T., \& Herrmann, C. S. (2012). Finite-Element Model Predicts Current Density Distribution for Clinical Applications of tDCS and tACS. Frontiers in Psychiatry, 3, 83.

Nitsche, M. A, Cohen, L. G., Wassermann, E. M., Priori, A., Lang, N., Antal, A., ... Pascual-Leone, A. (2008). Transcranial direct current stimulation: State of the art 2008. Brain Stimulation, 1(3), 206-23.

Nitsche, M. A., Doemkes, S., Karaköse, T., Antal, A., Liebetanz, D., Lang, N., ... Paulus, W. (2007). Shaping the effects of transcranial direct current stimulation of the human motor cortex. Journal of Neurophysiology, 97(4), 3109-17.

Oostendorp, T. F., Hengeveld, Y. A., Wolters, C. H., Stinstra, J., van Elswijk, G., \& Stegeman, D. F. (2008). Modeling transcranial DC stimulation. Conference Proceedings: Annual International Conference of the IEEE Engineering in Medicine and Biology Society, 4226-9.

Oostenveld, R., \& Praamstra, P. (2001). The five percent electrode system for high-resolution EEG and ERP measurements. Clinical Neurophysiology, 112(4), 713-9.

Pascual-Leone, A., Bartres-Faz, D., \& Keenan, J. P. (1999). Transcranial magnetic stimulation: studying the brain-behaviour relationship by induction of "virtual lesions". Philosophical Transactions of the Royal Society of London. Series B, Biological Sciences, 354(1387), 1229-38. 
Paulus, W. (2011). Transcranial electrical stimulation (tES - tDCS; tRNS, tACS) methods. Neuropsychological Rehabilitation, 21(5), 602-17.

Pawliczek, C. M., Derntl, B., Kellermann, T., Kohn, N., Gur, R. C., \& Habel, U. (2013). Inhibitory control and trait aggression: Neural and behavioral insights using the emotional stop signal task. Neurolmage, 79, 264-274.

Poreisz, C., Boros, K., Antal, A., \& Paulus, W. (2007). Safety aspects of transcranial direct current stimulation concerning healthy subjects and patients. Brain Research Bulletin, 72(4-6), 208-14.

Quay, H.C. (1998). The behavioral reward and inhibition system in childhood behavior disorder. In Bloomingdale, L.M. (Ed), Attention deficit disorder, Vol. 3: New research in attention, treatment, and psychopharmacology, 176-186. New York: Spektrum.

Raichle, M. E. (1994). Visualizing the mind. Scientific American, 270(4), 58-64.

Rampersad, S., Stegeman, D., \& Oostendorp, T. (2011). On handling the layered structure of the skull in transcranial direct current stimulation models. Conference Proceedings : Annual International Conference of the IEEE Engineering in Medicine and Biology Society, 1989-92.

Revelle, W., Humphreys, M. S., Simon, L., \& Gilliland, K. (1980). The interactive effect of personality, time of day, and caffeine: a test of the arousal model. Journal of Experimental Psychology. General, 109(1), 131.

Reynolds, S. M., \& Berridge, K. C. (2008). Emotional environments retune the valence of appetitive versus fearful functions in nucleus accumbens. Nature Neuroscience, 11(4), 423-5.

Roebroeck, A., Formisano, E., \& Goebel, R. (2005). Mapping directed influence over the brain using Granger causality and fMRI. Neurolmage, 25(1), 230-42.

Rossi, S., Hallett, M., Rossini, P. M., \& Pascual-Leone, A. (2009). Safety, ethical considerations, and application guidelines for the use of transcranial magnetic stimulation in clinical practice and research. Clinical Neurophysiology, 120(12), 2008-39.

Sack, A. T. (2006). Transcranial magnetic stimulation, causal structure-function mapping and networks of functional relevance. Current Opinion in Neurobiology, 16(5), 593-9.

Sack, A. T., Cohen Kadosh, R., Schuhmann, T., Moerel, M., Walsh, V., \& Goebel, R. (2009). Optimizing functional accuracy of TMS in cognitive studies: a comparison of methods. Journal of Cognitive Neuroscience, 21(2), 207-21.

Scheier, M. F., Buss, A. H., \& Buss, M. (1978). Self-Report of Aggressiveness, and Aggression. Journal of Research in Personality, 12, 133-140.

Simmonds, D. J., Pekar, J. J., \& Mostofsky, S. H. (2008). Meta-analysis of Go/No-go tasks demonstrating that fMRI activation associated with response inhibition is task-dependent. Neuropsychologia, 46(1), 22432.

Sparing, R., Hesse, M. D., \& Fink, G. R. (2010). Neuronavigation for transcranial magnetic stimulation (TMS): Where we are and where we are going. Cortex, 46(1), 118-20.

Sternberg, R. (1999). The nature of cognition. Cambridge (MA): MIT Press.

Swick, D., Ashley, V., \& Turken, U. (2011). Are the neural correlates of stopping and not going identical? Quantitative meta-analysis of two response inhibition tasks. Neurolmage, 56(3), 1655-65.

Terney, D., Chaieb, L., Moliadze, V., Antal, A., \& Paulus, W. (2008). Increasing human brain excitability by transcranial high-frequency random noise stimulation. The Journal of Neuroscience, 28(52), 14147-55.

Vigil-Colet, A., Ruiz-Pamies, M., Anguiano-Carrasco, C., \& Lorenzo-Seva, U. (2012). The impact of social desirability on psychometric measures of aggression. Psicothema, 24(2), 310-5.

Wagner, T., Fregni, F., Fecteau, S., Grodzinsky, A., Zahn, M., \& Pascual-Leone, A. (2007). Transcranial direct current stimulation: a computer-based human model study. Neurolmage, 35(3), 1113-24.

Wassermann, E. M. (1998). Risk and safety of repetitive transcranial magnetic stimulation: report and suggested guidelines from the International Workshop on the Safety of Repetitive Transcranial Magnetic Stimulation, June 5-7, 1996. Electroencephalography and Clinical Neurophysiology, 108(1), 1-16.

Williams, J. A., Imamura, M., \& Fregni, F. (2009). Updates on the use of Non-Invasive Brain Stimulation in physical and rehabilitation medicine. Journal of Rehabilitation Medicine, 41(5), 305-11. 



\title{
CHAPTER 2
}

A network approach to response inhibition

\author{
Dissociating functional connectivity of \\ neural components involved in action \\ restraint and action cancellation
}

"Every characteristic absence of spirituality, every piece of common vulgarity, is due to an inability to resist a stimulus - you have to react, you follow every impulse."

— Friedrich Nietzsche, Twilight of the Idols, 1889 
Based on: Dambacher, F., Sack, A. T., Lobbestael, J., Arntz, A., Brugman, S., \& Schuhmann, T. (2014). A network approach to response inhibition: dissociating functional connectivity of neural components involved in action restraint and action cancellation. The European Journal of Neuroscience, 39(5), 821-31.

We thank Katherine L. Wheat, Armin Heinecke, and Thomas C. Emmerling for their helpful support. 


\section{ABSTRACT}

The ability to inhibit action tendencies is vital for adaptive human behaviour. Various paradigms are supposed to assess action inhibition and are often used interchangeably. However, these paradigms are based on different conceptualizations (action restraint versus action cancellation) and the question arises, to what extent different conceptualizations of inhibitory processing are mirrored in a distinct neural activation pattern. We used functional Magnetic Resonance Imaging to investigate the neural correlates of action restraint vs. action cancellation. Analyses of local activity changes as well as network connectivity measures revealed a strong overlap of activation within a common action inhibition network including inferior frontal, pre-supplementary motor and thalamic brain areas as well as anterior cingulate cortex. Furthermore, our findings point to additional neural networks that are distinct for action restraint (i.e., right superior frontal gyrus, left middle frontal gyrus, and bilateral anterior cingulate cortex) and action cancellation (i.e., right middle frontal gyrus, posterior cingulate cortex, and parietal regions). Our connectivity analyses show that different inhibitory modalities largely rely on a task-independent global inhibition network within the brain. Furthermore, they suggest that the conceptually distinct inhibitory aspects of action restraint versus action cancellation also activate additional specific brain regions in a task-dependent manner. This has implications for the choice of tasks in an empirical setting, but is also relevant for various clinical contexts in which inhibition deficits are considered a diagnostic feature. 
Social interaction heavily relies on an actors' capacity to modify reactions and to inhibit pre-planned or automatic responses. Therefore, response (or action) inhibition, defined as the cognitive ability to withhold such reactions (Logan et al., 1997), is considered one of the key concepts in understanding the flexible and adaptive nature of human behaviour.

Various paradigms have been developed to assess response inhibition (Wöstmann et al., 2012), whereby the go/no-go task (GNGT) and the stop-signal task (SST) are the most prominent ones. Both paradigms share the task instruction to not respond to a certain configuration of stimuli and might, thus, revert to similar cognitive resources (Schachar et al., 2007). However, both paradigms are understood to employ different aspects of inhibitory processing (Aron et al., 2004; McNab et al., 2008; Rubia et al., 2001; Swick et al., 2011; Zheng, Oka, Bokura, \& Yamaguchi, 2008). Eagle and colleagues (2008) stated that in a GNGT a not yet initiated action has to be restrained. In contrast, in a SST already initiated responses have to be cancelled. Therefore, it is of interest to what extent such conceptual differences are mirrored by differences in task-related neural activity.

Brain imaging studies have consistently reported inhibition-related activity within mostly right-lateralized (Aron et al., 2004) ventral and inferior prefrontal cortex, presupplementary motor areas, and subcortical circuitries (Chambers et al., 2009). Only few studies directly compared neural activation in go/no-go and stop signal paradigms, focusing mainly on overlapping activation and identifying bi-hemispheric inferior, right middle frontal, and left anterior insula regions (McNab et al., 2008; Zheng et al., 2008) as the common locus of inhibitory processing. Rubia and colleagues (2001) reported left-lateralized prefrontal and parietal activation for action restraint and more righthemispheric activation during action cancellation. Sebastian and colleagues (2013) disentangled common and specific neural sub-processes of different inhibitory aspects. They found empirical evidence for a common inhibition network including inferior frontal cortex, pre-supplementary motor area, and parietal regions, but still emphasized that different aspects of action inhibition, i.e. interference inhibition and action cancellation, are discriminable on neural level. Also Swick and colleagues (2011) proposed in their comprehensive meta-analysis, that both tasks share a fundamental core network involving bilateral inferior frontal and pre-supplementary motor regions. Furthermore, they assumed an additional fronto-parietal network to be involved action restraint and a cingulo-opercular network in action cancellation.

However, the identification of common and distinct inhibitory neural networks and their respective brain connectivity measures were not directly addressed in any of the mentioned studies. A connectivity approach is indispensable in order to enable claims reaching beyond the mere description of co-activation of brain regions towards an understanding of their actual interaction. Therefore, we designed a functional imaging study in order to look at connectivity as well as local activity changes during response inhibition. Action restraint was expected to activate a fronto-parietal network while 
action cancellation was thought to activate a cingulo-opercular network. In addition to these task-dependent neural network patterns, we furthermore expected to also find a common right-lateralized inhibition network including inferior frontal and presupplementary motor areas.

\section{MATERIALS AND METHODS}

Participants and tasks. Nineteen healthy Dutch males volunteered for this study. One participant was excluded from further analysis because of technical problems during functional Magnetic Resonance Imaging (fMRI) data acquisition and one participant did not complete the experiment. Therefore, data of 17 (mean age $=28.4$; SD $=9.9$ ) participants were included in the data analysis. All participants gave their written informed consent for participation and were paid for taking part. The study was approved by the Ethical Commission of the Faculty of Psychology and Neuroscience at Maastricht University and conformed to the Code of Ethics of the World Medical Association (Declaration of Helsinki).

Participants had to complete two response inhibition tasks during the fMRI scan. Both task designs have been held as similar as possible in order to enable a valid comparison of their specific distinct inhibitory mechanisms. Furthermore, feasibility and comfortable task execution inside the fMRI scanner was considered to have participants perform with full concentration.

In order to elicit action restraint, a simple go/no-go motor response task was used (Fig.1A). Participants were instructed to respond as fast and accurately as possible to a go stimulus via button press, while they should not respond to a rare no-go stimulus. Go as well as no-go stimuli were presented for 200 ms followed by a fixation cross for a randomized interval of 1300,2800 , or 4300 ms.

In order to elicit action cancellation, a modified version of the stop-signal task (Logan et al., 1997) was used (Fig.1B). Participants were instructed to respond as fast and accurately as possible to a go stimulus via button press, while they should not respond to this same stimulus in the rare cases when it was followed by a visual stop-signal. In the go trials the go stimulus was presented for 200 ms followed by a fixation cross for a randomized interval of 1300,2800 , or $4300 \mathrm{~ms}$. In the stop trials the go stimulus was presented for $200 \mathrm{~ms}$ followed with a stimulus fixed onset asynchrony of $250 \mathrm{~ms}$ by a visual stop-signal presented for $300 \mathrm{~ms}$ and subsequently a fixation cross for a randomized interval of 950, 2450, or $3950 \mathrm{~ms}$. The fixed stop-signal delay was chosen to ensure optimal comparability between both paradigms and to keep the difficulty of the task at a level on which enough inhibition trials could be completed successfully. It has to be emphasized that this task design did not allow calculating stop signal reaction times within the framework of the independent race model (Verbruggen \& Logan, 2009). 


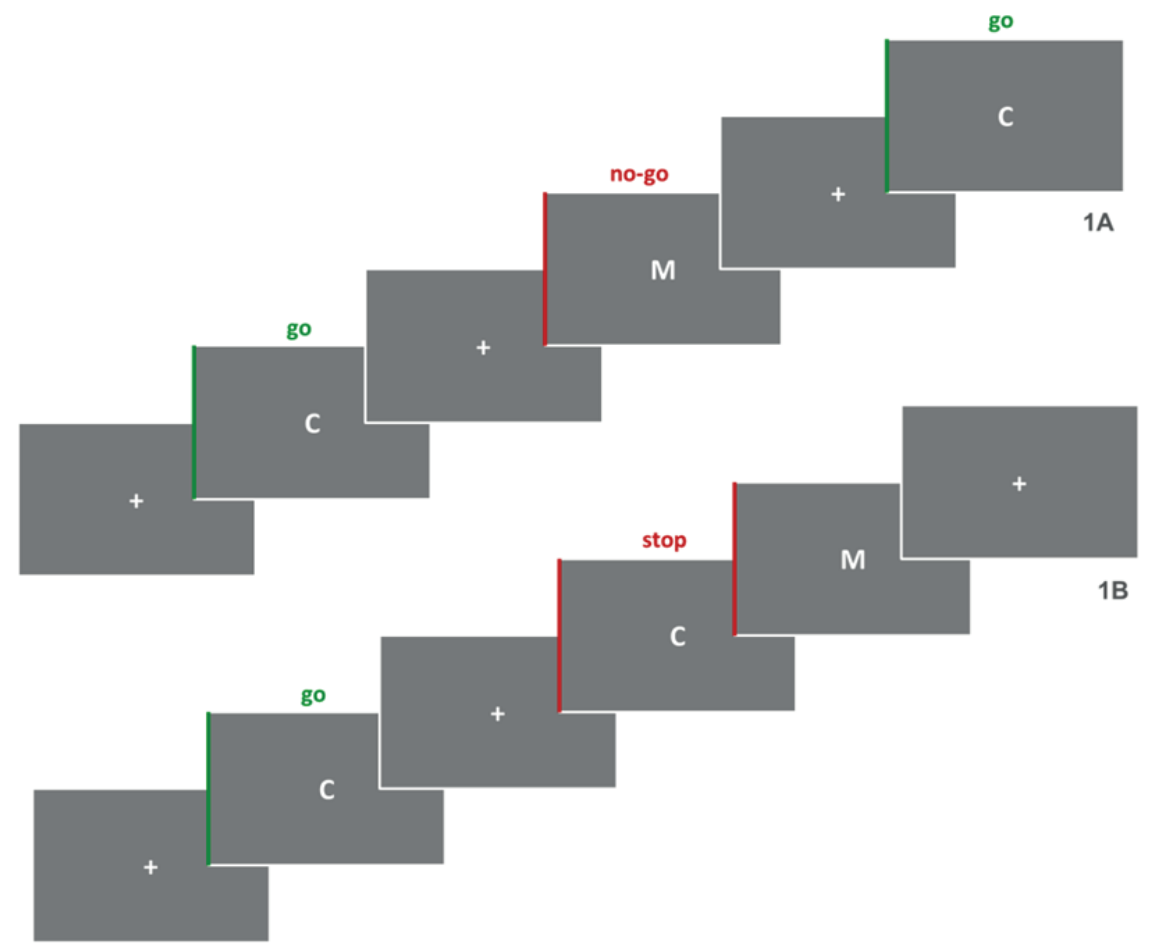

Fig.1. Task design. 1A) Go/no-go task (GNGT). Go trials colour-coded in green, no-go/stop trials colour-coded in red. 1B) Stop-signal task (SST). Go trials colour-coded in green, no-go/stop trials colour-coded in red.

No-go / stop events occurred in $25 \%$ of the 160 pseudo-randomized trials. The letters C and $\mathrm{M}$ were used as stimuli. Assigning a letter to the go and the no-go / stop condition was randomized between participants. After every 16 trials there was a short resting period of 19500 ms integrated into the task, where only a fixation cross was shown. Stimuli and fixation crosses were presented in white (RGB 255/255/255; Arial pt 24) on a grey background (RGB 125/125/125). Participants had to perform two runs consecutive of 160 trials for each task in randomized order, leading to a total of 320 trials (80 no-go / stop trials) per task and a scanning time of about 1.5 hours (approximately 10 minutes for each experimental run, plus acquisition of anatomical data, plus preparation). Task order was counterbalanced. After being instructed and before entering the fMRI scanner all participants had completed a training version of each task (including 64 trials per task). Stimuli were presented using Presentation software (Neurobehavioural Systems, Inc., Albany, USA).

Technical details and fMRI acquisition. Stimulus material was displayed onto a frosted screen, positioned at rear of the scanner bore, using an LCD projector (PLCXT11-16, Sanyo North American Corporation, SanDiego, USA). Responses were col- 
lected employing a standard MR compatible button box (LUMltouch keypads, Photon Control, Burnaby, Canada).

Using a 3 T Siemens Allegra MR Scanner structural (high resolution T1-weighted

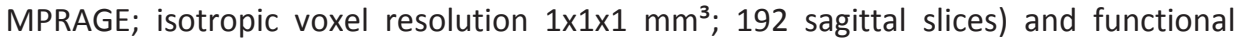
whole-brain (Gradient-Echo-EPI-sequence; TR=1500ms; TE $=28 \mathrm{~ms}$; FOV $=224 \mathrm{~mm}$; flip angle $=71^{\circ}$; matrix $=64 \times 64$; slice thickness $=3.5 \mathrm{~mm}$; distance factor $=10 \%$; 458 volumes per run) images were acquired. 27 oblique transversal slices of $3.5 \times 3.5 \times 3.5 \mathrm{~mm}$ voxels tilted $30^{\circ}$ relatively to the anterior-posterior commissure (AC-PC) plane were obtained in order to avoid signal dropout in frontal areas (Deichmann, Gottfried, Hutton, \& Turner, 2003).

FMRI analysis. Data analyses were performed using Brain Voyager QX 2.8 (Brain Innovation BV, Maastricht, Netherlands). The first five volumes of each functional run were discarded to allow for equilibrium effects. Preprocessing included 3D-motioncorrection (as implemented in Brain Voyager QX with trilinear / sinc interpolation and intra-session alignment to the 1st functional volume recorded after the individual anatomical scan), cubic spline slice scan time correction, and the application of a temporal high pass filter (GLM with Fourier basis set of 3 cycles sine/cosine per run including linear trend removal). Images were co-registered to the individual anatomical scans and normalization to Talairach stereotaxic space (Talairach \& Tournoux, 1988) Volume time courses were spatially smoothed using a $6 \mathrm{~mm}$ full width half maximum Gaussian kernel.

In order to establish activation patterns of the GNGT and the SST as well as their dissociative brain activity, event-related random effects group analyses were performed. A General Linear Model (GLM) was defined in order to analyze specific taskrelated activation patterns for no-go trials in the GNGT and specific activation patterns for stop trials in the SST. The GLM included four predictors (successful go and successful no-go trials in the GNGT, successful go and successful stop trials in the SST). Furthermore, commission errors on go trials (misses) and on no-go and stop trials (false alarms) were modeled in order to account for error variance. For each trial BOLDresponses during a time window of $1500 \mathrm{~ms}$ (one complete trial excluding the jitter) were modeled. Motion parameters were included as confound predictors in the regression analysis and the single regressors in the resulting matrix were tested in order to avoid multicollinearity. Statistical maps were created using a threshold of $p<.01$ corrected for multiple comparisons by means of cluster threshold level estimation analysis of 1000 Monte Carlo simulation iterations (Forman et al., 1995) resulting in a cluster threshold of 28 voxels. A conjunction analysis for successful no-go and stop trials (no-go ${ }^{\wedge}$ stop) was conducted to statistically evaluate the overlap (and only the overlap) of voxels significantly activated during both tasks and therefore involved in both tasks. Furthermore, a direct contrast analysis (stop > no-go) was performed to directly compare the two tasks and to examine the dissociative brain activity patterns between the no-go trials in the GNGT and the stop trials in the SST. 
Connectivity analysis and seed regions. In order to dissociate the overlapping and differential neural networks involved in both tasks, functional connectivity analyses were performed using Instantaneous Correlation Mapping (Goebel et al., 2003; Roebroeck et al., 2005). Thereby, we meant to investigate whether regions activated during action restraint and cancellation (in the GLM analyses) did indeed form a taskrelated functional network of orchestrated activity across distributed regions. This type of analysis requires the definition of seed regions. The task-related linear pairwise correlations were computed during the no-go and stop condition for each voxel between the average time course of the voxels in the seed region and the voxel time course.

The choice of seed regions was based on previous literature (Swick et al., 2011) and the concrete results of our GLM analysis. Thereby, we focused on three potential networks: a supposedly common inhibition network, a specific inhibition network for action restraint, and a specific inhibition network for action cancellation. Therefore, connectivity analyses were based on three (one for each network) different seed regions. In order to fulfill the criteria to be selected as seed regions, the regions had to be located within the hypothetical networks involved in action restraint and cancellation as suggested by Swick and colleagues (2011), and, furthermore, display significant neural activation in our GLM analysis. We chose the following seed regions:

- a common seed region in right anterior insula (as crucial node in a global inhibition network)

- an action restraint specific seed region in right frontal cortex (as crucial node in an action restraint fronto-parietal network)

- and an action cancellation specific seed region in posterior cingulate cortex (as crucial node in an action cancellation cingulo-opercular network).

The ROls within these seed regions were defined depending on the specific group random effects GLM activation, focusing on the 250-300 most significantly activated voxels in the three regions. The ROI on which connectivity analysis regarding a potential common inhibition system was based, was defined on the GLM map of the conjunction between successful action restraint and action cancellation (no-go ${ }^{\wedge}$ stop). The ROI on which connectivity analysis regarding a potential action restraint specific inhibition system was based, was defined on the GLM map of successful action restraint versus successful action cancellation (no-go > stop). The ROI on which connectivity analysis regarding a potential action cancellation specific inhibition system was based, was defined on the GLM map of successful action cancellation versus successful action restraint (stop > no-go).

Group level instantaneous correlation maps were computed by averaging all single subject maps and testing the average $T>0$. Cluster threshold level estimation analysis were performed with $\mathrm{p}<.0001$ and 1000 Monte Carlo simulation iterations in order to correct for multiple comparisons (Forman et al., 1995) resulting in a cluster threshold of five voxels. 


\section{RESULTS}

Behavioural data. Participants reacted significantly faster in the successful go trials for the GNGT compared to the SST (GNGT: MEAN=412.5ms / SD=46.9ms; SST: MEAN=505.6ms / SD=65.1ms; $\mathrm{t}=9.240 \mathrm{p}<.001$ ). False alarms (commission errors for inhibition trials) were more frequent in stop trials than in no-go trials (GNGT: MEAN=2.9 (3.6\% of all no-go trials) / SD=2.7; SST: MEAN=8.5 ( $10.6 \%$ of all stop trials) / $\mathrm{SD}=7.3 ; \mathrm{t}=3.394 \mathrm{p}<.05)$. This probably reflects a higher cognitive load due to more complex instructions in the SST. In both task relatively few misses (commission errors on go trials) occurred (GNGT: MEAN=1.4 (0.6\% of all go trials) / SD=2.2; SST: MEAN=1.1 ( $0.5 \%$ of all go trials) / $\mathrm{SD}=2.5 ; \mathrm{t}=-.206 \mathrm{p}>.05)$. As predicted by the independent race model (Verbruggen \& Logan, 2009), in the SST participants reacted significantly faster on false alarm trials than on successful go trials (false alarm trials: MEAN=412.5ms / $\mathrm{SD}=46.9 \mathrm{~ms}$; successful go trials: $\mathrm{MEAN}=505.6 \mathrm{~ms} / \mathrm{SD}=65.1 \mathrm{~ms} ; \mathrm{t}=6.042 \mathrm{p}=.000$ ).

GLM analysis. The GLM analysis of $\mathrm{fMRI}$ blood oxygenation level dependent (BOLD) responses during no-go and stop trials in which inhibition was successful compared to baseline (baseline=fixation; action restraint colour-coded in red, action cancellation colour-coded in blue), their conjunction (colour-coded in green), and their direct contrast (colour-coded in purple) are shown in figure 2 (for Talairach coordinates (TAL) of reported regions see table 1 ).

An increase of neural activity during both action restraint and action cancellation (no-go ${ }^{\wedge}$ stop / conjunction) occurs most prominently in right anterior insula (rAl) extending into right inferior frontal gyrus (rIFG). Additionally, pronounced changes of neural activation in pre-supplementary and supplementary motor area ((pre-)SMA) were observed. Activation in the left primary motor cortex increased during both nogo and stop trials, mirroring the strong urge to initiate a motor response with the right index finger that was though ultimately not completed. Furthermore, activation in parietal and inferior occipital regions increased significantly during both no-go and stop trials.

In addition, during successful action restraint in the GNGT (no-go versus baseline) the increase of activation in right superior frontal gyrus (SFG) showed to be marginally significant (random effects analysis $\mathrm{N}=17$, cluster threshold of 7 voxels; however, this cluster did not survive the cluster threshold level correction). Furthermore, superior temporal activation increased significantly.

No significant superior frontal activation was found for action cancellation in the SST (stop versus baseline). During stop trials activation in AI (extending into IFG) bilaterally showed a significant increase, whereby activation was more pronounced in the right inferior frontal regions. Furthermore, activation in the fusiform gyrus increased significantly in successful inhibition trials during SST execution. No such activation was found for the GNGT. 

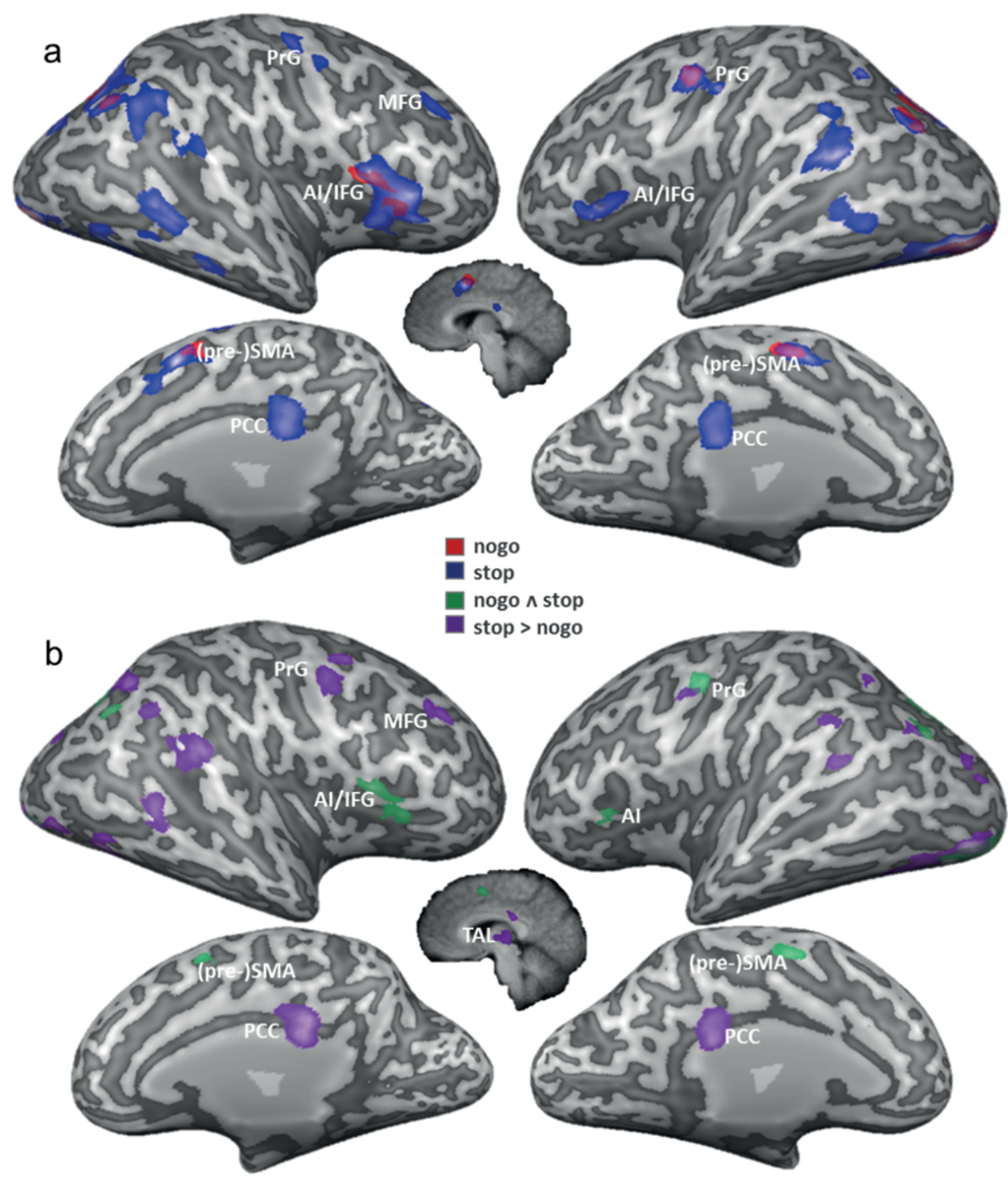

Fig.2. GLM maps. a) Increased cortical activation associated with successful inhibition during no-go trials vs. baseline (colour-coded in red, cluster threshold) and stop trials vs. baseline (colour-coded in blue). b) Conjunction analysis (no-go $\wedge$ stop) colour-coded in green and contrast analysis (stop trials $>$ no-go trials) colorcoded in purple. Statistical maps $p \leq .01$ cluster level threshold (CLT) corrected (cluster threshold of 28 voxels) Random Effects group Analysis (RFX) $n=17$. Abbreviations: PrG precentral gyrus, MFG middle frontal gyrus, Al anterior insula, IFG inferior frontal gyrus, (pre-)SMA (pre-)supplementary motor area, PCC posterior cingulate cortex, TAL thalamus. Neural activation overlaid over a single representative subject's brain. 
Tab.1. Talairach coordinates for regions activated in different analyses. Abbreviations: R right, L left.

\begin{tabular}{|c|c|c|c|c|}
\hline \multirow[b]{2}{*}{ Region } & & \multicolumn{3}{|c|}{ Talairach coordinates } \\
\hline & & $x$ & $y$ & $z$ \\
\hline \multicolumn{5}{|l|}{ No-go ^^ stop / conjunction } \\
\hline \multicolumn{5}{|l|}{ Frontal cortex } \\
\hline Anterior insula I & $\mathrm{R}$ & 32 & 24 & 10 \\
\hline Anterior insula II & $\mathrm{R}$ & 40 & 12 & 8 \\
\hline SMA/pre-SMA & $\mathrm{R}$ & 6 & 4 & 52 \\
\hline SMA/pre-SMA & $\mathrm{L}$ & -6 & -5 & 52 \\
\hline Pre-central gyrus & $\mathrm{L}$ & -46 & -7 & 44 \\
\hline \multicolumn{5}{|l|}{ Parietal cortex } \\
\hline Angular gyrus & $\mathrm{R}$ & 33 & -58 & 34 \\
\hline Precuneus & $\mathrm{R}$ & 32 & -68 & 38 \\
\hline Angular gyrus & $\mathrm{L}$ & -31 & -62 & 33 \\
\hline Precuneus & $\mathrm{L}$ & -31 & -68 & 38 \\
\hline \multicolumn{5}{|l|}{ Occipital cortex } \\
\hline Inferior occipital gyrus & $\mathrm{R}$ & 30 & -87 & -11 \\
\hline Inferior occipital gyrus & $\mathrm{L}$ & -33 & -87 & -11 \\
\hline \multicolumn{5}{|l|}{ No-go versus baseline } \\
\hline \multicolumn{5}{|l|}{ Frontal cortex } \\
\hline Superior frontal gyrus & $\mathrm{R}$ & 25 & 49 & 29 \\
\hline Anterior insula I & $\mathrm{R}$ & 32 & 24 & 10 \\
\hline Anterior insula II & $\mathrm{R}$ & 40 & 12 & 8 \\
\hline SMA/pre-SMA & $\mathrm{R}$ & 6 & 3 & 45 \\
\hline SMA/pre-SMA & $\mathrm{L}$ & -7 & -7 & 54 \\
\hline Pre-central gyrus & $\mathrm{L}$ & -46 & -7 & 44 \\
\hline \multicolumn{5}{|l|}{ Temporal cortex } \\
\hline Superior temporal gyrus & $\mathrm{R}$ & 49 & -26 & -5 \\
\hline \multicolumn{5}{|l|}{ Parietal cortex } \\
\hline Angular gyrus & $\mathrm{R}$ & 33 & -55 & 32 \\
\hline Precuneus & $\mathrm{R}$ & 34 & -66 & 42 \\
\hline Angular gyrus & $\mathrm{L}$ & -29 & -56 & 36 \\
\hline Precuneus & $\mathrm{L}$ & -29 & -66 & 36 \\
\hline \multicolumn{5}{|l|}{ Occipital cortex } \\
\hline Inferior occipital gyrus & $\mathrm{R}$ & 29 & -90 & -9 \\
\hline Inferior occipital gyrus & $\mathrm{L}$ & -30 & -88 & -11 \\
\hline \multicolumn{5}{|l|}{ Stop versus baseline } \\
\hline \multicolumn{5}{|l|}{ Frontal cortex } \\
\hline Middle frontal gyrus & $\mathrm{R}$ & 37 & 32 & 33 \\
\hline Anterior insula & $\mathrm{R}$ & 37 & 19 & 6 \\
\hline
\end{tabular}




\begin{tabular}{|c|c|c|c|c|}
\hline \multirow[b]{2}{*}{ Region } & & \multicolumn{3}{|c|}{ Talairach coordinates } \\
\hline & & $x$ & $y$ & $z$ \\
\hline Anterior insula & $\mathrm{L}$ & -35 & 22 & 5 \\
\hline SMA/pre-SMA & $\mathrm{R}$ & 6 & 8 & 51 \\
\hline SMA/pre-SMA & $\mathrm{L}$ & -5 & -1 & 50 \\
\hline Pre-central gyrus & $\mathrm{R}$ & 37 & -1 & 52 \\
\hline Pre-central gyrus & $\mathrm{L}$ & -42 & -7 & 37 \\
\hline \multicolumn{5}{|l|}{ Cingulate cortex } \\
\hline Posterior cingulate cortex & $\mathrm{R}$ & 4 & -26 & 26 \\
\hline Posterior cingulate cortex & L & -4 & -26 & 28 \\
\hline \multicolumn{5}{|l|}{ Temporal cortex } \\
\hline Superior temporal gyrus & $\mathrm{R}$ & 61 & -38 & 21 \\
\hline Superior temporal gyrus & $\mathrm{L}$ & -50 & -43 & 21 \\
\hline Fusiform gyrus & $\mathrm{R}$ & 45 & -61 & -8 \\
\hline Fusiform gyrus & $\mathrm{L}$ & -41 & -65 & -8 \\
\hline Middle temporal gyrus & $\mathrm{R}$ & 51 & -26 & -5 \\
\hline \multicolumn{5}{|l|}{ Parietal cortex } \\
\hline Precuneus & $\mathrm{R}$ & 33 & -68 & 35 \\
\hline Precuneus & $\mathrm{L}$ & -32 & -66 & 39 \\
\hline Inferior parietal lobe & $\mathrm{R}$ & 34 & -53 & 36 \\
\hline Inferior parietal lobe & $\mathrm{L}$ & -33 & -54 & 39 \\
\hline \multicolumn{5}{|l|}{ Occipital cortex } \\
\hline Inferior occipital gyrus & $\mathrm{R}$ & 33 & -84 & -10 \\
\hline Inferior occipital gyrus & $\mathrm{L}$ & -37 & -84 & -10 \\
\hline \multicolumn{5}{|l|}{ Stop > no-go / direct contrast } \\
\hline \multicolumn{5}{|l|}{ Frontal cortex } \\
\hline Middle frontal gyrus & $\mathrm{R}$ & 37 & 32 & 33 \\
\hline Pre-central gyrus & $\mathrm{R}$ & 44 & -2 & 45 \\
\hline \multicolumn{5}{|l|}{ Cingulate cortex } \\
\hline Posterior cingulate cortex & $\mathrm{R}$ & 4 & -26 & 26 \\
\hline Posterior cingulate cortex & $\mathrm{L}$ & -4 & -26 & 28 \\
\hline \multicolumn{5}{|l|}{ Temporal cortex } \\
\hline Superior temporal gyrus I & $\mathrm{R}$ & 61 & -38 & 21 \\
\hline Superior temporal gyrus II & $\mathrm{R}$ & 53 & -40 & 13 \\
\hline Superior temporal gyrus & $\mathrm{L}$ & -50 & -43 & 21 \\
\hline Middle temporal gyrus & $\mathrm{R}$ & 42 & -56 & -2 \\
\hline \multicolumn{5}{|l|}{ Parietal cortex } \\
\hline Precuneus & $\mathrm{R}$ & 27 & -72 & 29 \\
\hline Superior parietal lobe & $\mathrm{L}$ & -35 & -63 & 44 \\
\hline Inferior parietal lobe & $\mathrm{R}$ & 34 & -49 & 41 \\
\hline
\end{tabular}




\begin{tabular}{|c|c|c|c|c|}
\hline \multirow[b]{2}{*}{ Region } & & \multicolumn{3}{|c|}{ Talairach coordinates } \\
\hline & & $x$ & $y$ & $z$ \\
\hline Inferior parietal lobe & $\mathrm{L}$ & -33 & -54 & 39 \\
\hline \multicolumn{5}{|l|}{ Occipital cortex } \\
\hline Inferior occipital gyrus & $\mathrm{R}$ & 45 & -75 & 0 \\
\hline Inferior occipital gyrus & $\mathrm{L}$ & -37 & -84 & -10 \\
\hline \multicolumn{5}{|l|}{ Sub-cortical regions } \\
\hline Thalamus & $\mathrm{R}$ & 5 & -19 & 4 \\
\hline \multicolumn{5}{|c|}{ Common inhibition network GNGT } \\
\hline \multicolumn{5}{|l|}{ Frontal cortex } \\
\hline Anterior insula & $\mathrm{R}$ & 33 & 23 & 8 \\
\hline Anterior insula & $\mathrm{L}$ & -36 & 18 & 4 \\
\hline SMA/pre-SMA & $\mathrm{R}$ & 3 & -8 & 51 \\
\hline SMA/pre-SMA & $\mathrm{L}$ & -4 & -7 & 50 \\
\hline \multicolumn{5}{|l|}{ Cingulate cortex } \\
\hline Anterior cingulate cortex & $\mathrm{L}$ & -4 & 10 & 36 \\
\hline \multicolumn{5}{|l|}{ Occipital cortex } \\
\hline Lingual gyrus & $\mathrm{R}$ & 9 & -70 & 0 \\
\hline Lingual gyrus & $\mathrm{L}$ & -6 & -74 & -4 \\
\hline \multicolumn{5}{|l|}{ Sub-cortical regions } \\
\hline Thalamus & $\mathrm{R}$ & 8 & -17 & 4 \\
\hline \multicolumn{5}{|c|}{ Common inhibition network SST } \\
\hline \multicolumn{5}{|l|}{ Frontal cortex } \\
\hline Anterior insula & $\mathrm{R}$ & 33 & 23 & 8 \\
\hline Anterior insula & $\mathrm{L}$ & -37 & 20 & 1 \\
\hline SMA/pre-SMA & $\mathrm{R}$ & 2 & 2 & 49 \\
\hline SMA/pre-SMA & $\mathrm{L}$ & -4 & -2 & 49 \\
\hline \multicolumn{5}{|l|}{ Cingulate cortex } \\
\hline Anterior cingulate cortex & $\mathrm{L}$ & -1 & 16 & 36 \\
\hline \multicolumn{5}{|l|}{ Occipital cortex } \\
\hline Lingual gyrus & $\mathrm{R}$ & 6 & -68 & 4 \\
\hline Lingual gyrus & $\mathrm{L}$ & -9 & -78 & 4 \\
\hline \multicolumn{5}{|l|}{ Sub-cortical regions } \\
\hline Caudate & $\mathrm{L}$ & -6 & 5 & 7 \\
\hline Putamen/globus pallidus & $\mathrm{R}$ & 28 & -7 & 7 \\
\hline Thalamus & $\mathrm{L}$ & -8 & -17 & 7 \\
\hline \multicolumn{5}{|c|}{ Common inhibition network GNGT \& SST } \\
\hline \multicolumn{5}{|l|}{ Frontal cortex } \\
\hline Anterior insula & $\mathrm{R}$ & 33 & 23 & 8 \\
\hline Anterior insula & $\mathrm{L}$ & -38 & 20 & 1 \\
\hline
\end{tabular}




\begin{tabular}{|c|c|c|c|c|}
\hline \multirow[b]{2}{*}{ Region } & & \multicolumn{3}{|c|}{ Talairach coordinates } \\
\hline & & $x$ & $y$ & $z$ \\
\hline SMA/pre-SMA & $\mathrm{R}$ & 4 & 2 & 46 \\
\hline SMA/pre-SMA & L & -6 & -2 & 46 \\
\hline Pre-central gyrus & $\mathrm{R}$ & 36 & -11 & 57 \\
\hline \multicolumn{5}{|l|}{ Cingulate cortex } \\
\hline Anterior cingulate cortex & $\mathrm{R}$ & 6 & 13 & 34 \\
\hline Anterior cingulate cortex & $\mathrm{L}$ & -6 & 13 & 37 \\
\hline \multicolumn{5}{|l|}{ Temporal cortex } \\
\hline Superior temporal gyrus & $\mathrm{L}$ & -56 & -27 & 12 \\
\hline \multicolumn{5}{|l|}{ Occipital cortex } \\
\hline Lingual gyrus & $\mathrm{R}$ & 8 & -68 & 5 \\
\hline Lingual gyrus & L & -8 & -80 & 5 \\
\hline \multicolumn{5}{|l|}{ Sub-cortical regions } \\
\hline Caudate & L & -13 & -2 & 13 \\
\hline Putamen/globus pallidus & $\mathrm{R}$ & 29 & -7 & 5 \\
\hline Thalamus & $\mathrm{R}$ & 5 & -18 & 6 \\
\hline Thalamus & $\mathrm{L}$ & -8 & -18 & 6 \\
\hline \multicolumn{5}{|c|}{ Specific inhibition network GNGT } \\
\hline \multicolumn{5}{|l|}{ Frontal cortex } \\
\hline Superior frontal gyrus & $\mathrm{R}$ & 24 & 49 & 30 \\
\hline Middle frontal gyrus & L & -26 & 37 & 36 \\
\hline \multicolumn{5}{|l|}{ Cingulate cortex } \\
\hline Anterior cingulate cortex & $\mathrm{R}$ & 4 & 23 & 33 \\
\hline Anterior cingulate cortex & $\mathrm{L}$ & -5 & 25 & 33 \\
\hline \multicolumn{5}{|l|}{ Specific inhibition network SST } \\
\hline \multicolumn{5}{|l|}{ Frontal cortex } \\
\hline Middle frontal gyrus & $\mathrm{R}$ & 40 & 44 & 20 \\
\hline \multicolumn{5}{|l|}{ Cingulate cortex } \\
\hline Posterior cingulate cortex & $\mathrm{R}$ & 5 & -26 & 27 \\
\hline Posterior cingulate cortex & L & -6 & -26 & 27 \\
\hline \multicolumn{5}{|l|}{ Parietal cortex } \\
\hline Inferior parietal lobule & $\mathrm{R}$ & 39 & -56 & 42 \\
\hline Precuneus & $\mathrm{R}$ & 10 & -51 & 46 \\
\hline Precuneus & L & -5 & -50 & 46 \\
\hline \multicolumn{5}{|l|}{ Occipital cortex } \\
\hline Middle occipital gyrus & $\mathrm{R}$ & 29 & -79 & 21 \\
\hline
\end{tabular}


For trials in which inhibition was successful the direct statistical contrast between both aspects of action inhibition (stop > no-go / direct contrast) revealed no significant increase of BOLD-signal in specific brain areas for action restraint (GNGT). For action cancellation (SST) the analysis of the direct contrast revealed SST-specific activation in right middle frontal gyrus ( $\mathrm{MMFG}$ ), bilateral posterior cingulate cortex (PCC), and right lateralized thalamus. Furthermore, activation in bilateral parietal and temporal areas as well as inferior occipital gyrus was more pronounced for action cancellation.

Based on the described analyses and the hypothetical network suggested by Swick and colleagues (2011), three ROls were defined as seed regions for functional connectivity analyses (see section "Connectivity analysis, choice and definition of seed regions"): a common ROI in right Al (TAL: $x 33, y 23, z 8)$ in order to find evidence for a common inhibition network, an action restraint specific ROI in right SFG (TAL: x24, y49, $z 30)$ in order to find evidence for an action restraint specific fronto-parietal network, and an action cancellation specific ROI in PCC (TAL: x0, y-26, z26) in order to find evidence for an action cancellation specific cingulo-opercular network.

Functional connectivity analysis. Additionally to the standard GLM fMRI analysis, we conducted functional connectivity analyses in order to enable the specification of overlapping and differential functional networks activated by different aspects of action inhibition (Tab.1, Fig.3). Instantaneous correlation analyses showed that for both aspects of inhibitory processing, a network including bilateral inferior frontal regions, (pre-)SMA, anterior cingulate cortex (ACC), thalamus, and occipital areas was activated. This common inhibition network was found for the aggregated data of successful no-go and stop trials (Fig.3, colour-coded in green) and also independently for both paradigms (Fig.3, action restraint colour-coded in red, action cancellation colour-coded in blue). Although visual inspection of the instantaneous correlation maps suggests that subcortical areas might be more strongly involved in the common inhibition network for action cancellation, the direct contrast of the found network for action restraint and action cancellation (connectivity within common network during stop trials > connectivity within common network during no-go trials) did not reveal any statistically significant differences in neural activation. 


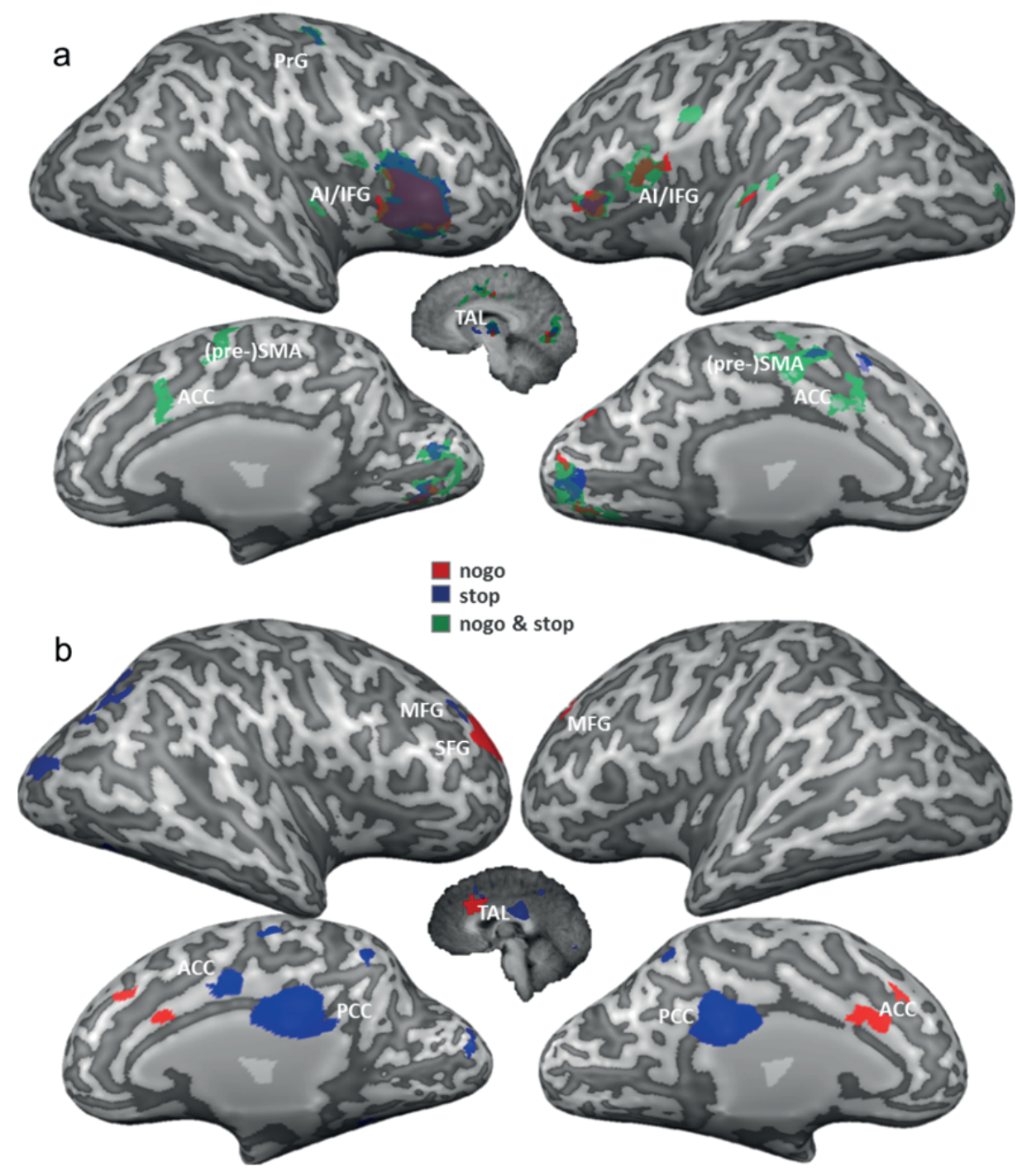

Fig.3. Functional connectivity within inhibitory networks activated during successful inhibition. a) Common inhibition network for conjunction of no-go and stop trials colour-coded in green, common network for GNGT colour-coded in red (seed region right AI); common network for SST colour-coded in blue (seed region right AI). b) Specific network for GNGT colour-coded in red (seed region right SFG); specific network for SST colour-coded in blue (seed region PCC). Statistical maps $p \leq .0001$ CLT corrected (cluster threshold of 5 voxels) RFX N=17. Abbreviations: Al anterior insula, IFG inferior frontal gyrus, (pre-)SMA (pre-)supplementary motor area, ACC anterior cingulate cortex, TAL thalamus, SFG superior frontal gyrus, MFG middle frontal gyrus, PCC posterior cingulate gyrus. Neural activation overlaid over a single representative subject's brain.

In addition to the common inhibition network, functional connectivity measures showed that during action restraint a network including right superior frontal regions, left middle frontal regions, and anterior cingulate cortex was activated (Fig.3, colour- 
coded in red). No such functional network was found during action cancellation. In contrast, for action cancellation posterior cingulate cortex, right middle frontal gyrus, parietal and occipital regions were found to be functionally related (Fig.3, colourcoded in blue). No such network was found to be activated during action restraint.

\section{DISCUSSION}

Employing a within-subject within-session event-related fMRI design, analyzing functional network connectivity as well as local activity changes, we investigated to what extent the neural correlates of two different aspects of response inhibition, action restraint and action cancellation, rely on a common and/or task dependent network. We expected to find both a common inhibition system within the brain as well as different activation patterns and neural networks involved in action restraint and action cancellation. Specifically, we expected action restraint to activate a task-specific fronto-parietal network and action cancellation to activate a task-specific cingulo-opercular network.

\section{A common inhibition network and its lateralization}

A common inhibition network. In accordance with our hypotheses, we found right and left anterior insula regions, which extend into inferior frontal gyrus, presupplementary, and supplementary motor areas, to be activated during successful inhibition in both action restraint (assessed by the GNGT) and action cancellation (assessed by the SST). Our connectivity analyses, moreover, showed task-related functional connectivity between bilateral inferior frontal regions, (pre-) SMA, and thalamic regions. Therefore, our data support the notion that both paradigms share a common pattern of neural activation. We interpret these findings as direct evidence for a common, or core task set, inhibition network across different modalities of response inhibition, as suggested previously (Swick et al., 2011). These brain regions have previously been investigated and associated with inhibitory processing in neuroimaging studies (Chambers et al., 2009; Swick et al., 2011), as well as human lesion (Aron, Fletcher, Bullmore, Sahakian, \& Robbins, 2003), EEG (Enriquez-Geppert, Konrad, Pantev, \& Huster, 2010), and brain stimulation (Chambers et al., 2006, 2007; Majid, Cai, George, Verbruggen, \& Aron, 2012) research.

During successful inhibition, we find an interaction between inferior frontal regions, (pre-)SMA, and thalamus. This connects to work by Aron (Aron, 2011) who presented a hypothetical network account suggesting that such an IFG-SMA-THALAMUS network is involved in global reactive stopping. For the SST, Duann and colleagues (2009) investigated directed connectivity by means of Granger Causality Mapping. They found reciprocal connectivity between inferior frontal cortex and (pre-)SMA. 
Furthermore, they showed that (pre-)SMA mediates response inhibition through its connectivity with primary motor cortex via the basal ganglia circuitry, while inferior frontal cortex interconnects to this system indirectly through its connectivity with (preISMA. Recently, Zandbelt and colleagues (2013) revealed interactions between inferior frontal cortex, pre-supplementary motor area, striatum and motor cortex using an approach combining repetitive transcranial magnetic brain stimulation and fMRI.

During successful inhibition, inferior frontal regions seem to not only interact with (pre-)SMA, but also with thalamus. Previous literature emphasized thalamic involvement in an inhibition network also with respect to the anatomical setup of connecting fiber tracts (Aron, Behrens, Smith, Frank, \& Poldrack, 2007). Such involvement was mainly shown to work via suppression mechanisms: Thalamic activation should be released during execution of motor action, whereas it should be suppressed during its inhibition (Aron, 2011; Duann et al., 2009). The current findings emphasize that thalamic regions play a crucial role in the processing of motor responses and their inhibition. However, it has to be considered that mere functional connectivity measures cannot enable any interpretation regarding the directivity of such an involvement. More elaborate studies employing directed connectivity measures are needed to clarify the precise interplay of involved regions within a global inhibition network across different inhibitory modalities.

Notably, the described common inhibition network seems to be crucial not only for the SST but for both tasks independently and also for their conjunction. Future research may, therefore, need to consider that different response inhibition paradigms show significant overlap in their neural networks. Furthermore, it would be of interest to investigate how action restraint and cancellation are conceptually equivalent to reactive inhibition or incorporate additionally also proactive components (Zandbelt, Bloemendaal, Neggers, Kahn, \& Vink, 2012; Zandbelt, van Buuren, Kahn, \& Vink, 2011; Zandbelt, Bloemendaal, Neggers, Kahn, \& Vink, 2013; Zandbelt \& Vink, 2010) and whether the global inhibition network we find transfers to these other conceptualizations of action inhibition.

The current study found pronounced activation of inferior frontal regions during successful inhibition for both action restraint and action cancellation; however, although this activation extends into IFG, our findings indicate that the statistical "hotspot" for successful inhibition clearly lies in anterior insula rather than IFG. This finding implies that IFG and anterior insula may form a functional entity during inhibitory processing, and that anterior insula may be the most crucial brain region for different forms of response inhibition. This is in contrast to the consistent emphasis on the role of rIFG in the response inhibition literature (Aron et al., 2004), which has been described as being a core region for response inhibition that is involved across different response modalities (Chikazoe, Konishi, Asari, Jimura, \& Miyashita, 2007). However, in line with the current findings, Swick et al. (2011) found the most prominent overlap of inferior frontal activation to be located in anterior insula in their meta-analysis 
of 42 studies of GNGT and SST. They suggest two possible reasons for the disagreement over IFG or anterior insula as the focus of activation during response inhibition. First, the close proximity of IFG and anterior insula to each other could result in mislabelling of fMRI activation in either IFG or anterior insula. Secondly, extensive spatial smoothing of functional imaging data could blur distinct regions in such way that activation in IFG would be smoothed into anterior insula and vice versa. Swick and colleagues suggest that it is especially likely that activation foci in group level statistical maps would appear in anterior insula. However, despite the strength of this argument, in our data we see the strongest activation focus for both tasks in anterior insula, even for single subject and unsmoothed group data. This emphasis on the role of the anterior insula alongside IFG activation in response inhibition research follows the example of Sharp et al. (2010), who labeled their focus of activation as "IFG / insula", and acknowledges the importance of anterior insula in inhibition processing and its relation to IFG. It would be of interest to examine whether these two regions could be functionally discriminated in the context of inhibition research.

Lateralization of the common inhibition network. We find stronger right hemispheric activation in the bilateral areas of interest and also a greater number of right hemisphere unilateral regions of activation for both tasks. Therefore, our findings suggest that the brain regions involved in response inhibition tasks are asymmetrically distributed towards the right hemisphere. This is in line with most response inhibition literature, independent of the paradigm used (Aron et al., 2004; Chambers et al., 2009; Swick et al., 2011). The evidence presented here is in contrast to the notion of Rubia et al. (2001) that a GNGT would show greater involvement of the left hemisphere, while a SST would involve more right hemispheric regions.

Despite the clear right lateralization of activation, our findings also show that brain activation is not exclusively located in the right hemisphere, indicating a role for the left hemisphere in inhibitory processing. Several other studies have shown explicit involvement of left IFG / left anterior insula in inhibition tasks (Boehler, Appelbaum, Krebs, Hopf, \& Woldorff, 2010; Swick, Ashley, \& Turken, 2008), suggesting that lefthemispheric regions play a role in response inhibition, though to a lesser extent. Therefore, future investigations are needed to clarify the specific division of labor between the two hemispheres for action inhibition.

\section{Distinct neural networks underlying action restraint and action cancellation}

The neural correlates of action restraint. Contrary to our prediction, the direct contrast between stop trials in the SST and no-go trials in the GNGT does not show any significant differential activation for action restraint (as assessed by the GNGT). We do not find specific GNGT related increases of neural activation in rMFG and right inferior parietal lobule / precuneus, as would be expected based on the meta-analysis by Swick et al. (2011). The lack of specific neural activation related to action restraint compared 
to action cancellation in our data is in line with the findings of Sebastian and colleagues (2013). They attribute this zero-finding to the fact that they presented action-restrainttrials interleaved with inference-inhibition- and action-cancellation-trials. However, we find the same results using two time-wise entirely unrelated tasks. Therefore, the question arises whether or not action restraint or withholding might be a sub form of action cancellation activating no or very few specific neuronal components additional to a global inhibition system.

On a more descriptive level, we find a prefrontal cluster of activation slightly superior to rMFG around Brodmann area (BA) 10 in the GLM analysis of successful no-go trials versus baseline that we do not find for action cancellation. The functional connectivity we found between right superior frontal regions, left middle frontal regions, and ACC emphasizes the role of these frontal regions for cognitive processing during action restraint. However, we failed to find convincing empirical evidence in favor of a GNGT-specific fronto-parietal network as suggested by Swick et al. (2011). We find some frontal areas being marginally important for GNGT execution, but no indication of their task-specific connectivity to parietal regions.

In our data, only superior frontal activation was specifically marginally significant for action restraint. However, in response inhibition literature there is still no consensus on which specific middle and/or superior frontal cortical structures are crucial for inhibitory processing. Simmonds et al. (2008) report a superior frontal region (BA10) similar to the one we find together with BA9 in their activation likelihood estimation over 11 studies investigating the GNGT. Swick et al. (2011) report as a result of their activation likelihood estimation over 21 studies investing the GNGT a middle frontal area (BA9) exclusively as the most crucial specific prefrontal area activated during the GNGT. The role of middle and superior frontal areas for inhibition is still not clearly defined and this might motivate a closer look into the precise distribution of specific neural activity in prefrontal areas especially during action restraint.

The neural correlates of action cancellation. For action cancellation (as assessed by the SST), we find a well-pronounced task-specific neural activation pattern. Hence, besides the common network of inhibition described above, action cancellation specifically activates PCC and thalamic regions in the direct contrast analysis between SST and GNGT. These regions have been associated with functions such as task set control, salience monitoring, and performance monitoring (Swick et al., 2011). Furthermore, our data shows increased activity in right middle frontal and bilateral parietal areas specifically for action cancellation. This activation pattern could suggest the involvement of a right lateralized fronto-parietal neural component similar to the dorsal attentional system (Corbetta, Patel, \& Shulman, 2008) or the executive control system (Seeley et al., 2007), both of which include top-down adaptive cognitive control as one of their central functions. Higher false alarms rates and longer reaction times measured during the SST compared to the GNGT (see behavioural results) suggest that the SST might call for a significantly higher cognitive load or might be simply more difficult. 
This interpretation could also account for the vaster and more pronounced taskrelated neural activation we find for the SST compared to the GNGT.

In our study a specific task design was employed to optimally compare the two paradigms. To do so, a fixed stop signal delay was used in the SST. This resulted in a significantly longer reaction times in SST- compared to GNGT-performance and one could assume that participants just waited for the stop signal and thereby transformed the SST simply into a slower GNGT. However, the significantly higher error rate in the SST as well as the fact that we found differential neural activity and networks underlying both paradigms might speak against this interpretation. Nonetheless, it would be of value to develop comparable designs for GNGT and SST that still allow for an implementation of variable stop signal delays in order to gain an even more defined assessment of the underlying differential mechanisms.

Our functional connectivity analysis reveals that, during successful inhibition of stop trials, activity in PCC is not correlated with activity in thalamic regions as predicted, but rather with rMFG, parietal, and occipital regions. Instead of being task-specific for action cancellation, our data thus suggest that thalamic regions are an integral component of a common inhibition network relevant during both action cancelation and action restraint. Therefore, the notion of a cingulo-opercular network activated in the stop-signal paradigm is supported by our data on a descriptive level, but not with respect to functional connectivity.

\section{Limitations}

When interpreting our findings, it is important to emphasize that the question of how to label processes underlying the stopping of certain actions is not yet resolved. The label "inhibition" may often not be an adequate description of the function of a brain region found to be active during response inhibition paradigms.

In the current study task-related neural activity and functional connectivity was assessed looking at activity changes during no-go and stop events and their contrasts compared to baseline. Therefore, we cannot interpret our findings as inhibitionspecific, but rather as task-specific. Claims can be made concerning the common and distinct networks recruited for the two tasks, but specificity to inhibitory processes cannot be concluded based on solely these results. It is debated about which contrast to look at for inhibition-specific activity (Swick et al., 2011) and additional options to the comparison against baseline would be comparing successful inhibition to false alarms or go events. Our design did not provide a sufficient number of false alarm trials to allow such statistical comparisons. Directly contrasting no-go and stop events to go events, on the other hand, did not reveal any significant results. This might be due to power issues. Essentially, we had to focus on the comparison to baseline which has to be seen as a rather sub-optimal choice of contrasts in response inhibition research (Swick et al., 2011). Our hypotheses focused on a task comparison and, thus, on 
differential task-related activity during inhibitory processing rather than the isolation of one specific form of inhibition. Furthermore, the type of connectivity analysis employed investigates neural activity during a certain time window rather than in contrast to other time courses. Therefore, the choice to base our analyses on the comparison against baseline is justified. However, it has to be emphasized that activation found might not be specific to action restraint or action cancellation, but could reflect other processes (e.g. response preparation). Further investigations have to clarify in how far the results bear up to a more specific choice of contrasts.

Furthermore, there are several considerations to take into account, when thinking about assigning certain functions to the involved regions. While (pre-)SMA and thalamic regions seem to be specifically involved in the mechanisms underlying the withdrawal of motor action (Swick et al., 2011), the exact role of inferior frontal brain structures is not clear. Variations in task design of go/no-go and stop-signal paradigms as well as the addition of control trials that can tap into processes such as action selection or the choice of alternative action plans may specifically be capable of shedding light on this important question. For example, Verbruggen and colleagues (2010) showed that the disruption of neural tissue in ventral rIFG by thetaburst transcranial magnetic brain stimulation did affected both stop-signal trials in which subjects had to withhold their response and dual-signal trials in which subjects had to execute an additional response. This finding suggests that when the cognitive system finds itself confronted with alternative competing plans, inferior frontal regions might serve as a higher-order control of updating and selecting the respective action plans, rather than underlying specifically their simple inhibition. Schall and Godlove (2012) point out that - when taking into account studies not focusing on inhibition only but also other cognitive processes - the function of inferior frontal cortex might rather be labeled as attention capture modulating with stimulus unexpectedness. These described functions of inferior frontal activity are similar to those discussed with regard to anterior insula: while classically understood as a tool of interoceptive awareness (Craig, 2009), anterior insula functions include the selection of appropriate responses, cognitive control, maintenance of task set, and focal attention (Vinod Menon \& Uddin, 2010; Nelson et al., 2010). Other authors have emphasized the role of right inferior frontal regions in attentional capture (Sharp et al., 2010) and attentional control (Hampshire, Chamberlain, Monti, Duncan, \& Owen, 2010). All of these aspects are crucial for the successful inhibitory processing of any kind.

Due to this evidence, we cannot conclusively label the function of the brain regions we find to be associated with action restrain and action cancellation as "inhibition". This is partly also to be attributed to the limitations of using fMRI as rather coarse method to study brain activation related to inhibition. Ultimately, the spatial resolution of imaging methods has to be combined with a time-wise more precise approach. For instance, in the context of SST research, Schall and Godlove (2012) emphasize the importance of looking at the modulation of activity in certain brain regions with re- 
spect to time components inherent to the task (e.g., stop-signal reaction time in the SST). Further research is needed to clarify the detailed allocation of tasks between involved brain structures in inhibitory processing. However, activation in inferior frontal brain areas, in particular, is certainly an integral component of every process associated with inhibition. Therefore, this activation seems to be fundamental to response inhibition.

\section{CONCLUSION AND IMPLICATIONS}

On a behavioural level, action restraint and action cancellation share the task instruction not to respond to a certain configuration of stimuli. Our findings show that they rely on a common network of neural activation including bilateral inferior frontal regions, (pre-)SMA, and thalamic regions. However, our findings also point to additional neural components that are distinct to action restraint (i.e., rSFG and ACC) or action cancellation (i.e., rMFG and PCC). We therefore conclude that response inhibition related neural activity across different inhibitory aspects does refer to a common inhibition system within the brain, but is in part also task dependent.

It might be that the strict dichotomous view of action restraint versus action cancellation as two independent dimensions of action inhibition needs revision. Instead of being conceptualized dichotomously, the two concepts might rather combine independent inhibitory processing with a fundamental shared cognitive inhibition system. Our findings of common, as well as specific, inhibitory neural components would support such a claim.

The findings of the present study suggest that the decision to use specific inhibition tasks in future neuroscientific research should be based on theoretical considerations that take into account the conceptual as well as the neural aspects of the hypotheses to be investigated. Furthermore, it is clear that in clinical settings the choice to include response inhibition paradigms as elements in diagnostic batteries should be made carefully. Therefore, with respect to the diagnostics of specific clinical disorders, it should be carefully reviewed which of the distinct inhibition aspects are relevant. In this way, research like this can contribute to disorder- (or even criteria-) specific tailored assessment and (perhaps in the very far future) alteration or treatment. 


\section{CHAPTER 2}

Aron, A. R. (2011). From reactive to proactive and selective control: developing a richer model for stopping inappropriate responses. Biological Psychiatry, 69(12), e55-68.

Aron, A. R., Behrens, T. E., Smith, S., Frank, M. J., \& Poldrack, R. A. (2007). Triangulating a cognitive control network using diffusion-weighted magnetic resonance imaging (MRI) and functional MRI. The Journal of Neuroscience, 27(14), 3743-52.

Aron, A. R., Fletcher, P. C., Bullmore, E. T., Sahakian, B. J., \& Robbins, T. W. (2003). Stop-signal inhibition disrupted by damage to right inferior frontal gyrus in humans. Nature Neuroscience, 6(2), 115-6.

Aron, A. R., Robbins, T. W., \& Poldrack, R. A. (2004). Inhibition and the right inferior frontal cortex. Trends in Cognitive Sciences, 8(4), 170-7.

Boehler, C. N., Appelbaum, L. G., Krebs, R. M., Hopf, J. M., \& Woldorff, M. G. (2010). Pinning down response inhibition in the brain: Conjunction analyses of the Stop-signal task. Neurolmage, 52(4), 1621-32.

Chambers, C. D., Bellgrove, M. a, Stokes, M. G., Henderson, T. R., Garavan, H., Robertson, I. H., ... Mattingley, J. B. (2006). Executive "brake failure" following deactivation of human frontal lobe. Journal of Cognitive Neuroscience, 18(3), 444-55.

Chambers, C. D., Bellgrove, M. A., Gould, I. C., English, T., Garavan, H., McNaught, E., ... Mattingley, J. B. (2007). Dissociable mechanisms of cognitive control in prefrontal and premotor cortex. Journal of Neurophysiology, 98(6), 3638-47.

Chambers, C. D., Garavan, H., \& Bellgrove, M. A. (2009). Insights into the neural basis of response inhibition from cognitive and clinical neuroscience. Neuroscience and Biobehavioral Reviews, 33(5), 631-46.

Chikazoe, J., Konishi, S., Asari, T., Jimura, K., \& Miyashita, Y. (2007). Activation of right inferior frontal gyrus during response inhibition across response modalities. Journal of Cognitive Neuroscience, 19(1), 69-80.

Corbetta, M., Patel, G., \& Shulman, G. L. (2008). The reorienting system of the human brain: from environment to theory of mind. Neuron, 58(3), 306-24.

Craig, A. D. B. (2009). How do you feel - now? The anterior insula and human awareness. Nature Reviews. Neuroscience, 10(1), 59-70.

Deichmann, R., Gottfried, J. A., Hutton, C., \& Turner, R. (2003). Optimized EPI for fMRI studies of the orbitofrontal cortex. Neurolmage, 19(2), 430-41.

Duann, J.-R., Ide, J. S., Luo, X., \& Li, C. R. (2009). Functional connectivity delineates distinct roles of the inferior frontal cortex and presupplementary motor area in stop signal inhibition. The Journal of Neuroscience, 29(32), 10171-9.

Eagle, D. M., Bari, A., \& Robbins, T. W. (2008). The neuropsychopharmacology of action inhibition: crossspecies translation of the stop-signal and go/no-go tasks. Psychopharmacology, 199(3), 439-56.

Enriquez-Geppert, S., Konrad, C., Pantev, C., \& Huster, R. J. (2010). Conflict and inhibition differentially affect the N200/P300 complex in a combined go/nogo and stop-signal task. Neurolmage, 51(2), 877-87.

Forman, S. D., Cohen, J. D., Fitzgerald, M., Eddy, W. F., Mintun, M. A., \& Noll, D. C. (1995). Improved assessment of significant activation in functional magnetic resonance imaging (fMRI): use of a cluster-size threshold. Magnetic Resonance in Medicine, 33(5), 636-47.

Hampshire, A., Chamberlain, S. R., Monti, M. M., Duncan, J., \& Owen, A. M. (2010). The role of the right inferior frontal gyrus: inhibition and attentional control. Neurolmage, 50(3), 1313-9.

Logan, G. (1997). Impulsivity and inhibitory control. Psychological Science, 8(1), 60-64.

Majid, D. S. A., Cai, W., George, J. S., Verbruggen, F., \& Aron, A. R. (2012). Transcranial magnetic stimulation reveals dissociable mechanisms for global versus selective corticomotor suppression underlying the stopping of action. Cerebral Cortex, 22(2), 363-71.

McNab, F., Leroux, G., Strand, F., Thorell, L., Bergman, S., \& Klingberg, T. (2008). Common and unique components of inhibition and working memory: an fMRI, within-subjects investigation. Neuropsychologia, 46(11), 2668-82.

Menon, V., \& Uddin, L. Q. (2010). Saliency, switching, attention and control: a network model of insula function. Brain Structure \& Function, 214(5-6), 655-67.

Nelson, S. M., Dosenbach, N. U. F., Cohen, A. L., Wheeler, M. E., Schlaggar, B. L., \& Petersen, S. E. (2010). Role of the anterior insula in task-level control and focal attention. Brain Structure \& Function, 214(56), 669-80. 
Rubia, K., Russell, T., Overmeyer, S., Brammer, M. J., Bullmore, E. T., Sharma, T., ... Taylor, E. (2001). Mapping motor inhibition: conjunctive brain activations across different versions of go/no-go and stop tasks. Neurolmage, 13(2), 250-61.

Schachar, R., Logan, G. D., Robaey, P., Chen, S., Ickowicz, A., \& Barr, C. (2007). Restraint and cancellation: multiple inhibition deficits in attention deficit hyperactivity disorder. Journal of Abnormal Child Psychology, 35(2), 229-38.

Schall, J. D., \& Godlove, D. C. (2012). Current advances and pressing problems in studies of stopping. Current Opinion in Neurobiology, 22(6), 1012-21.

Sebastian, A., Pohl, M. F., Klöppel, S., Feige, B., Lange, T., Stahl, C., ... Tüscher, O. (2013). Disentangling common and specific neural subprocesses of response inhibition. Neurolmage, 64, 601-15.

Seeley, W. W., Menon, V., Schatzberg, A. F., Keller, J., Glover, G. H., Kenna, H., ... Greicius, M. D. (2007). Dissociable intrinsic connectivity networks for salience processing and executive control. The Journal of Neuroscience, 27(9), 2349-56.

Sharp, D. J., Bonnelle, V., De Boissezon, X., Beckmann, C. F., James, S. G., Patel, M. C., \& Mehta, M. A. (2010). Distinct frontal systems for response inhibition, attentional capture, and error processing. National Academy of Sciences of the United States of America, 107(13), 6106-11.

Talairach, J., \& Tournoux, P. (1988). Co-planar stereotaxic atlas of the human brain: an approach to medical cerebral imaging. 

CHAPTER 3 The role of right prefrontal and medial cortex in response inhibition

\section{Interfering with action restraint and action cancellation using transcranial magnetic brain stimulation}

"Every impulse of feeling should be guided by reason; and, in my opinion, exertion should always be in proportion to what is required."

- Jane Austen, Pride and Prejudice, 1813 
Based on: Dambacher, F., Sack, A. T., Lobbestael, J., Arntz, A., Brugman, S., \& Schuhmann, T. (2014). The role of right prefrontal and medial cortex in response inhibition: interfering with action restraint and action cancellation using transcranial magnetic brain stimulation. Journal of Cognitive Neuroscience, 26(8), 1775-84.

We thank Thomas C. Emmerling, Jeannette Boschma, and Jan Schepers for their helpful support. 


\section{ABSTRACT}

The ability of inhibiting impulsive urges is paramount for human behaviour. Such successful response inhibition has consistently been associated with activity in prefrontal cortex. The current study aims to unravel the differential involvement of different areas within right prefrontal cortex for successful action restraint versus action cancellation. These two conceptually different aspects of action inhibition were measured with a go/no-go task (action restraint) and a stop-signal task (action cancellation). Localization of relevant prefrontal activation was based on functional Magnetic Resonance Imaging (fMRI) data. Significant task-related activation during successful action restraint was localized for each participant individually in right anterior insula (rAl), right superior frontal gyrus (rSFG), and pre-supplementary motor area (pre-SMA). Activation during successful action cancellation was localized in right anterior insula (rAl), right middle frontal gyrus (rMFG), and pre-supplementary motor area (pre-SMA). Subsequently, fMRI-guided continuous thetaburst stimulation (cTBS) was applied to these regions. Results showed that the disruption of neural activity in rAl reduced both the ability to restrain (go/no-go) and cancel (stop-signal) responses. In contrast, cTBSinduced disruption of the rSFG specifically impaired the ability to restrain from responding (go/no-go), while leaving the ability for action cancellation largely intact. Stimulation applied to rMFG and pre-SMA did not affect inhibitory processing in neither of the two tasks. These findings provide a more comprehensive perspective on the role of prefrontal cortex in inhibition and cognitive control. The results emphasize the role of inferior frontal regions for global inhibition, whereas superior frontal regions seem to be specifically relevant for successful action restraint. 
Self-control or the ability to select, adapt, and withhold behavioural responses is crucial to human functioning. However, everyday life examples in non-clinical as well as clinical contexts demonstrate how easily the ability to restrain or cancel automatic or preplanned reactions can break down. Neuroscience contributed to this discussion by starting to unravel which neurocorrelates are involved in the failure of inhibitory processing, whereby functional magnetic resonance imaging (fMRI) has repeatedly emphasized the role of prefrontal cortex in controlled behaviour and in self-regulation failure (Heatherton \& Wagner, 2011; Earl K Miller, 2000). One facet of self-regulation is response (or action) inhibition. Response inhibition, defined as the cognitive ability to withhold any planned or automatic reaction (Logan et al., 1997), is considered one of the key concepts in understanding the flexible and adaptive nature of human behaviour. Neuroimaging studies investigating the involvement of prefrontal neural components in response inhibition mainly used simple motor response inhibition paradigms, focusing on either of two aspects of inhibitory processing: action restraint as measured in go/no-go tasks (GNGTs) and action cancellation as measured in stop signal tasks (SSTs). These imaging studies consistently reported task-related activity within inferior frontal gyrus (IFG) or anterior insula (AI), pre-supplementary motor area (pre-SMA), and subcortical circuitries involving thalamic regions and the striatum (Chambers et al., 2009; Swick et al., 2011). Within the prefrontal cortex, mainly dorsolateral prefrontal cortex, middle frontal gyrus (MFG), and superior frontal gyrus (SFG) have been emphasized (Simmonds et al., 2008; Swick et al., 2011). In most studies an asymmetric distribution of neural activity towards the right hemisphere was found to play a role in inhibition (Aron et al., 2004).

Similarly, the neurocorrelates of response inhibition have also received attention in brain stimulation research. Noninvasive brain stimulation (NIBS) techniques such as transcranial magnetic brain stimulation (TMS) and transcranial direct current stimulation (tDCS) can provide further insights into the functional architecture of the response inhibition system in the brain, offering a complimentary methodological perspective to functional imaging approaches (Sack \& Linden, 2003). For example the inhibitory processing in a SST was shown to be impaired by event related TMS over pre-SMA (Chen, Muggleton, Tzeng, Hung, \& Juan, 2009) and frontal eye field (Muggleton, Chen, Tzeng, Hung, \& Juan, 2010). Repetitive TMS over right IFG compared to a sham TMS condition was shown to reduce inhibitory control in a SST, while no effects of stimulation on right MFG, right angular gyrus, right and left dorsal premotor cortex, and left IFG were observed (Chambers et al., 2006, 2007). In line with these findings, Verbruggen and colleagues (2010) showed that the disruption of neural tissue in specifically the ventral part of the right IFG by means of cTBS (continuous theta burst stimulation) impaired response inhibition in a stop signal paradigm. In a tDCS study, Hsu and colleagues (2011) revealed that cathodal stimulation (inhibiting neural activity) over pre-SMA impaired, while anodal stimulation (enhancing neural activity) elevated the ability to successfully inhibit responses in a SST. Jacobson and colleagues (2011) showed that 
while cathodal tDCS did not have an effect on response inhibition, anodal tDCS over the rIFG improved inhibitory processing in a SST. Furthermore, effects of cathodal tDCS over right dorsolateral prefrontal cortex diminishing successful inhibition in a GNGT have been demonstrated (Beeli, Casutt, Baumgartner, \& Jäncke, 2008).

While converging evidence of these functional brain stimulation studies indicate a causal role of rIFG in successful response inhibition, most of the above mentioned studies focused exclusively on the SST as a measure of action cancellation. Whether or not the same or other neural structures within prefrontal cortex are relevant for action restraint was not addressed in any of these previous studies. Moreover, while most studies did compare right IFG with other regions within right and left hemisphere, a systematic single subject based comparison of the differential functional relevance of several distinct sub-regions within right prefrontal cortex for successful action cancellation and/or action restraint is still missing.

The current study was designed to investigate the neural components involved in global response inhibition, as well as action restraint and action cancellation in particular. Virtual lesions were induced in several functional sub-regions within right prefrontal cortex and pre-SMA. CTBS, a repetitive patterned TMS protocol with which long lasting after effects can be achieved with relatively short periods of stimulation, was used. Thereby a 40 seconds train including 600 pulses of uninterrupted TBS ( 3 pulses of $50 \mathrm{~Hz}$ repeated every $200 \mathrm{~ms}$ ) is delivered (Huang et al., 2005). The specific target regions were localized for each individual subject based on functional imaging data. The differential effect of brain stimulation on three different prefrontal areas (rAI, rSFG, rMFG) and pre-SMA was systematically investigated. Individual fMRI-guided neuronavigation ensured that the individual "hotspots" of activations in every single participant were reliable targeted across several sessions. Behavioural effects of inducing neural disruptions within the various target regions were examined independently for action restraint (measured with a GNGT) and action cancellation (measured with a SST). 


\section{MATERIALS AND METHODS}

Participants. All participants ( $\mathrm{N}=11$; mean age in years $=27, \mathrm{SD}=7.27$ ) were native Dutch speakers, had normal or corrected-to-normal vision, and had no history of neurological or psychiatric disorders. They received medical approval from an independent physician for participation and gave their written informed consent after being introduced to the procedure. The study was approved by the local Medical Ethical Committee.

Experimental design. Participants took part in one training session and five experimental sessions. During the training session they were introduced to the TMS laboratories and the tasks they had to perform. Furthermore, their individual active motor threshold was determined. In each of the experimental sessions participants received cTBS (Huang et al., 2005) on one of four target sites or sham stimulation in a semicounterbalanced order. RSFG, rMFG, rAI, and pre-SMA were identified as target sites based on individual fMRI data (details presented below). Sham TMS was delivered on a target site located in the middle of the four empirical target sites in order to keep nonneural TMS effects constant (Duecker \& Sack, 2013). Therefore, Talairach coordinates of the four sites were averaged and transferred back to native space in order define a sham TMS target site. This procedure ensured sham TMS to be an optimally matched control condition for each specific empirical target site, especially with respect to the mimicking of the auditory sensation accompanying cTBS.

Paradigms. In order to access response inhibition two paradigms were employed: a go/no-go task (GNGT) and a stop signal task (SST). The tasks designs were comparable in order to enable the direct comparison of the outcome measures.

In order to elicit action restraint, a simple go/no-go motor response task was employed (Fig.1A). Participants were instructed to respond as fast and accurately as possible to a frequent go stimulus via button press with the right index finger, while they should not respond to a rare no-go stimulus. Go as well as no-go stimuli were presented for $100 \mathrm{msec}$. Inter-trial intervals were randomly varied among five levels $(650,750$, 850,950 , or $1050 \mathrm{msec}$ ) in order to avoid expectancy effects.

In order to elicit action cancellation, a modified stop signal task (Logan et al., 1997) was employed (Fig.1B). Participants were instructed to respond as fast and accurately as possible to a go stimulus via button press with the right index finger, while they should not respond to the very same stimulus in the rare cases when it was followed by a visual stop signal. No dual choice reaction time task was implemented in order to keep the design of the two tasks as similar as possible. This means that the go stimulus was presented in all trials and the stop stimulus was presented following the go stimulus only during stop trials. In the go trials the go stimulus was presented for $100 \mathrm{msec}$. In the stop trials the go stimulus was presented for $100 \mathrm{msec}$ followed with a stimulus onset asynchrony of $150,200,250,300$, or 350 msec by a visual stop signal presented 
for $80 \mathrm{msec}$. Inter trial intervals were jittered (1050 and $1250 \mathrm{msec}$ ) in order to avoid expectancy effects.

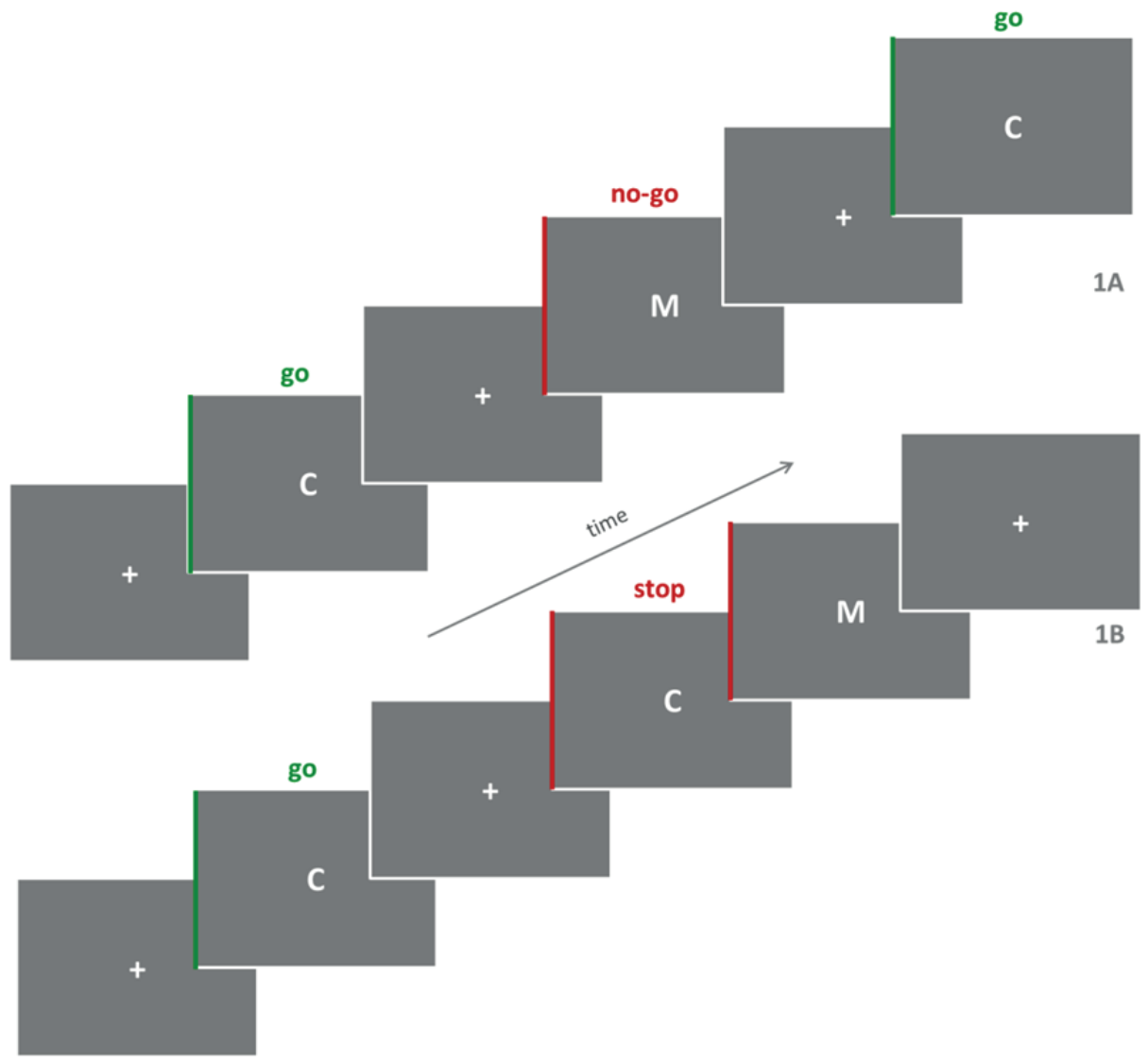

Fig.1. Task design. Go trials colour-coded in green, no-go / stop trials colour-coded in red. 1A) Go/no-go task (GNGT): Participants were instructed to respond as fast and accurately as possible to a frequent go stimulus (in this case a C) via button press, while they should not respond to a rare no-go stimulus (in this case an M). 1B) Stop signal task (SST): Participants were instructed to respond as fast and accurately as possible to a go stimulus (in this case a C) via button press, while they should not respond to this same stimulus in the rare cases when it was followed by a visual stop signal (in this case an $\mathrm{M}$ ).

The letters $\mathrm{C}$ and $\mathrm{M}$ were used as stimuli, since they do not have any linguistic association with the concept of "stopping". The assigned letter to the go respectively no-go / stop condition was randomized between participants. Stimuli and fixation crosses were presented in white (RGB 255/255/255; Arial pt 24) on a grey background (RGB $125 / 125 / 125)$. For each task, participants had to complete 5 blocks of 64 trials including $25 \%$ inhibition trials. Go and no-go / stop trials were pseudo-randomized (one of four trials was an inhibition trial) in order to prevent randomizations including trains of more than two consecutive inhibition trials. This design led to a total of 320 trials ( 80 
inhibition trials) per task. After each block participants received feedback on their mean reaction times for go trials, their number of misses in go trials, and their percentage of false alarms in inhibition trials. During the training session participants were familiarized with the tasks and our procedure and had to complete a training version of each task. Stimuli were presented using Presentation software (Neurobehavioural Systems, Inc., Albany, USA). The order of the two tasks within one session was counterbalanced within and semi-counterbalanced between participants.

Localization of TMS target regions. Previous to their participation in the current experiment all participants underwent structural (high resolution T1-weighted MPRAGE; isotropic voxel resolution $1 \times 1 \times 1 \mathrm{~mm}^{3} ; 192$ sagittal slices) and functional whole-brain (Gradient-Echo-EPI-sequence; TR=1500msec; TE $=28 \mathrm{msec}$; $F O V=224 \mathrm{~mm}$; flip angle $=71^{\circ}$; matrix $=64 \times 64$; slice thickness $=3.5 \mathrm{~mm}$; distance factor $=10 \%$; 458 volumes per run) imaging in a 3 Tesla Siemens Allegra Scanner. All fMRI analyses and 3D surface reconstructions were performed with Brain Voyager QX (Brain Innovation BV, Maastricht, Netherlands). For 17 participants random effects group analyses ( $p<.001$, cluster level threshold corrected) of successful inhibition in two fMRI adapted versions of the exact same tasks as described above were conducted. Functional imaging revealed three right lateralized frontal brain regions as being mainly activated during action restraint and/or action cancellation (rSFG for the GNGT, rMFG for the SST; rAI/IFG and pre-SMA for both tasks; Fig.2). Eleven participants from the sample were medically eligible to undergo TMS and agreed to come back for further sessions. For them the described regions were localized on single subject maps choosing the statistically most significant voxels of individual activation within the regions. The established target regions were transformed into surface clusters and projected onto the individual 3D surface reconstruction of each participant's right hemisphere (Fig.3; for specific individual Talairach coordinates see Tab.1). The average distance from the skull surface to the target site was $50 \mathrm{~mm}$ for $\mathrm{rAl}, 56 \mathrm{~mm}$ for pre-SMA, $33 \mathrm{~mm}$ for $\mathrm{rMFG}$, and $34 \mathrm{~mm}$ for rSFG.

TMS stimulation. Biphasic TMS pulses were applied using the Medtronic MagPro X100 stimulator (Medtronic Functional Diagnostics A/S, Skovlunde, Denmark, maximum stimulator output approximately 1.9 Tesla and $150 \mathrm{~A} / \mu \mathrm{s})$ and a figure-of-eight coil (MCB70; with the inner and outer radii of the two coil loops $=1.2 \mathrm{~cm}$ and $5.4 \mathrm{~cm}$, respectively). The coil was manually held tangentially to the skull, oriented perpendicularly to the gyrus on which the target site was located. The heads of the participants were co-registered to their structural brain imaging data using the online visualization function of Brain Voyager TMS Neuronavigator (Brain Innovation BV, Maastricht, The Netherlands). This allowed targeting brain areas precisely based on functional imaging data projected onto the 3D surface reconstructions of the participants' right hemispheres and monitoring the coil position while stimulating. CTBS was applied at $100 \%$ individual active motor threshold (ranging from 25\% to $36 \%$ of maximum stimulator output) for 40 seconds. Active motor threshold was determined by the observation of 
movement method as proposed by Varnava and colleagues (Varnava, Stokes, \& Chambers, 2011). For sham stimulation a placebo figure-of eight TMS coil (MC-P-B70 Placebo) was used. In the sham condition the neuronavigation procedure and all other stimulation parameters were equal to the real TMS conditions.

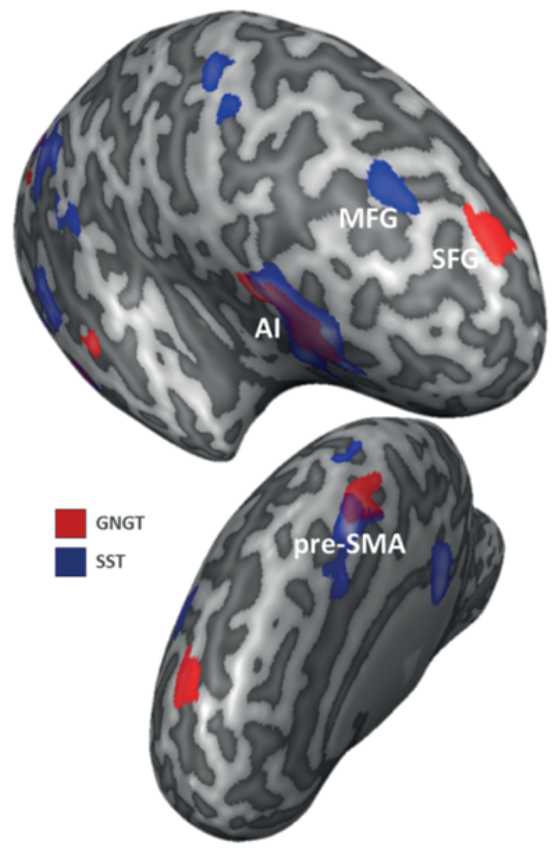

Fig.2. Increased cortical activation associated with successful inhibition during no-go (colour-coded in red) and stop (colour-coded in blue) trials. Lateral and medial view of an inflated right hemisphere. Statistical maps $\mathrm{p} \leq .001$ RFX $\mathrm{n}=17$. Abbreviations: GNGT go/nogo task, SST stop signal task, SFG superior frontal gyrus, MFG middle frontal gyrus, Al anterior insula, pre-SMA pre-supplementary motor area.

Tab.1. Talairach coordinates of individual brain stimulation target sites for every participant. Abbreviations: rAl right anterior insula, pre-SMA pre-supplementary motor area, rMFG right middle frontal gyrus, rSFG right superior frontal gyrus.

\begin{tabular}{|c|c|c|c|c|}
\hline & $r A l$ & pre-SMA & $r M F G$ & $r S F G$ \\
\hline Participant & $x y z$ & $x y z$ & $x y z$ & $x y z$ \\
\hline Group ( $n=17)$ & 33249 & 3549 & 373234 & 214943 \\
\hline 1 & 39154 & 5650 & 273635 & 205836 \\
\hline 2 & 40161 & $2-558$ & 443336 & 184450 \\
\hline 3 & 321510 & 6045 & 413234 & 175237 \\
\hline 4 & 37209 & $5-250$ & 313332 & 204635 \\
\hline 5 & 28228 & 5554 & 362633 & 165144 \\
\hline 6 & 372011 & 1559 & 423534 & 225343 \\
\hline 7 & 34263 & 4252 & 312334 & 124342 \\
\hline 8 & $3813-5$ & 8449 & 353922 & 215437 \\
\hline 9 & 391412 & 5549 & 343034 & 214043 \\
\hline 10 & 331910 & $4-454$ & 313038 & 234838 \\
\hline
\end{tabular}




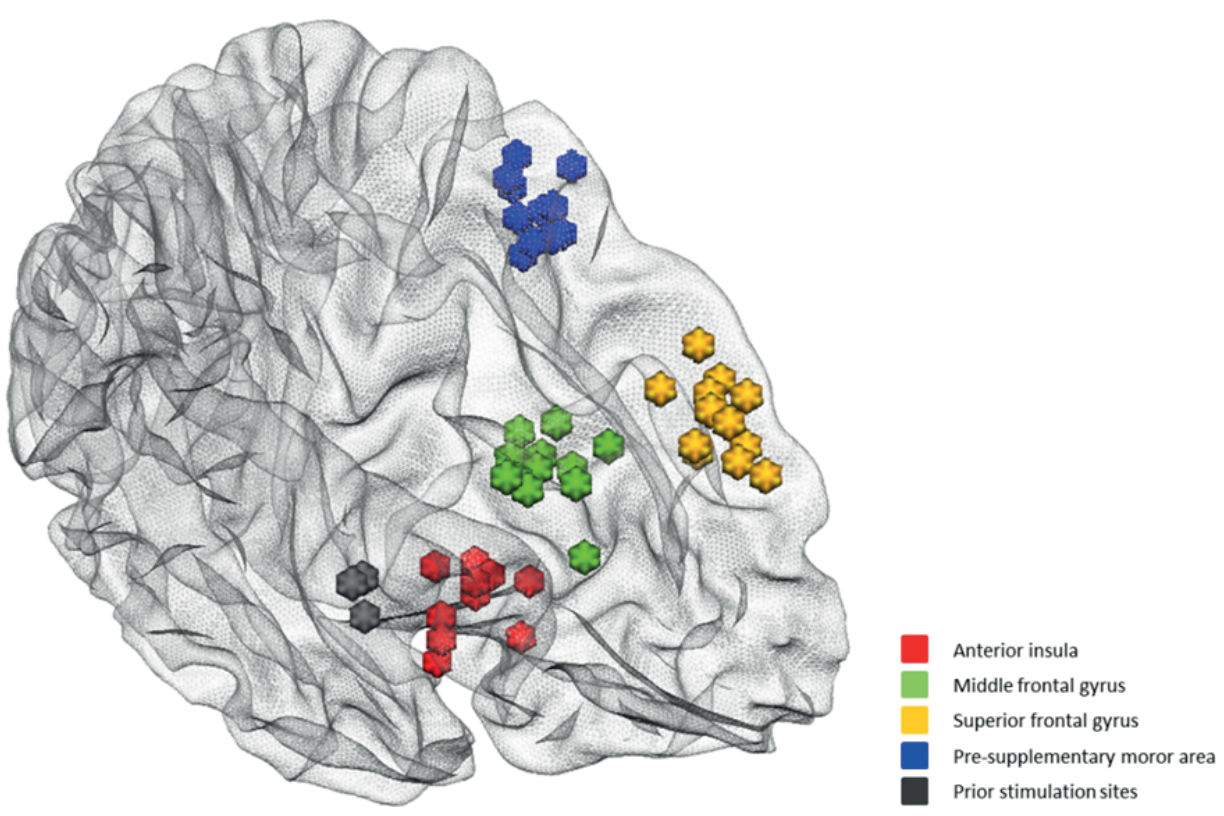

Fig.3. Individual fMRI-based brain stimulation target sites for eighth participant. Stimulation sites for which an effect of TMS on action cancellation could be demonstrated in prior studies (Chambers et al., 2006, 2007; Verbruggen et al., 2010) are shown colour-coded in grey.

Statistical analysis of behavioural data. One participant did not complete one experimental session, because of uncomfortable twitches in the face muscles. Therefore, this subject was excluded from the analyses. Ten participants completed all six sessions. Two participants had to be excluded due to behavioural false alarm pattern incompatible with the given task instruction (one participant showed false alarm rates on the GNGT entirely opposite to all other participants with an outlier analysis identifying his differential values as being $>1$ SD from the mean, while a second participant showed a false alarm rate on inhibition trials in the SST of $100 \%$ at baseline).Therefore, data of 8 participants was included in our analysis. Our sample size was evaluated by a power analysis based on the average effect sizes revealed by Chambers and colleagues (2006), Chambers and colleagues (2007), and Verbruggen and colleagues (2009) and the ratio to which a functionally guided localization of TMS target regions increases statistical power compared to a conventional approach (Sack et al., 2009). The power analysis was performed using G-power software 3.1.5 (Faul, Erdfelder, Lang, \& Buchner, 2007; assumptions: $\alpha 0.05,1-\beta 0.95)$.

For every participant and both tasks, mean reaction time, misses in go trials, and false alarms in inhibition trials were calculated. For the SST additionally the mean stop signal reaction time (SSRT) was estimated according to the horse-race model (Band, van der Molen, \& Logan, 2003). SSRT was defined per participant as the difference 
between stop signal delay at $50 \%$ correct inhibition and the median reaction time. All further statistical analyses were performed using SPSS19 (IBM Statistics, USA). In order to examine statistical differences in performance with respect to the five TMS conditions, a general linear mixed model analysis including the 4 stimulation sites as dummy coded variables with the sham condition as the reference variable was performed. Thereby, four a priori comparisons (each stimulation site compared to sham condition) could be tested. Mixed models provide an approach to repeated measures data in the framework of regression analyses. Order effects with respect to the individual sessions for false alarm rates were tested in a one-factorial analysis of variance with repeated measures.

\section{RESULTS}

The presented analyses are based on data of 8 participants. In the neutral (sham stimulation) condition participants reacted faster in the successful go trials for the GNGT compared to the SST (GNGT: $301.06 \pm 14.65 \mathrm{~ms}$; SST: $455.25 \pm 44.96 \mathrm{~ms}$ ). False alarms (commission errors on inhibition trials) were more frequent in stop trials than in no-go trials (GNGT: $16.00 \pm 3.42$; SST: $33.63 \pm 5.89$ ). In both tasks relatively few misses (omission errors on go trials) occurred (GNGT: $5.13 \pm 3.15$; SST: $2.38 \pm 0.82$ ). In the SST, an average SSRT of $166.52 \mathrm{~ms}$ was observed (SD = 102.77). However, no perfect positive linear relationship between false alarm rates and length of SSD (more false alarms the longer the SSD) as foreseen based on the simulations by Band and colleagues (2003) was found in our data. Furthermore, an erroneous estimation of SSRT might have been caused by the fact that we did not employ a dual choice RT paradigm as is often done in SST research. Therefore, the interpretability of SSRT in our study is questionable. Focusing on the significantly longer reaction times in SST- compared to GNGT-performance, one could assume that participants just waited for the stop signal and thereby transformed the SST simply into a slower GNGT. This is known to distort SSRT estimation (Verbruggen, Chambers, \& Logan, 2013). However, the significantly higher error rate in the SST as well as the fact that we find differential TMS results for both paradigms speaks against this interpretation. 


\section{false alarm rates for inhibition trials}

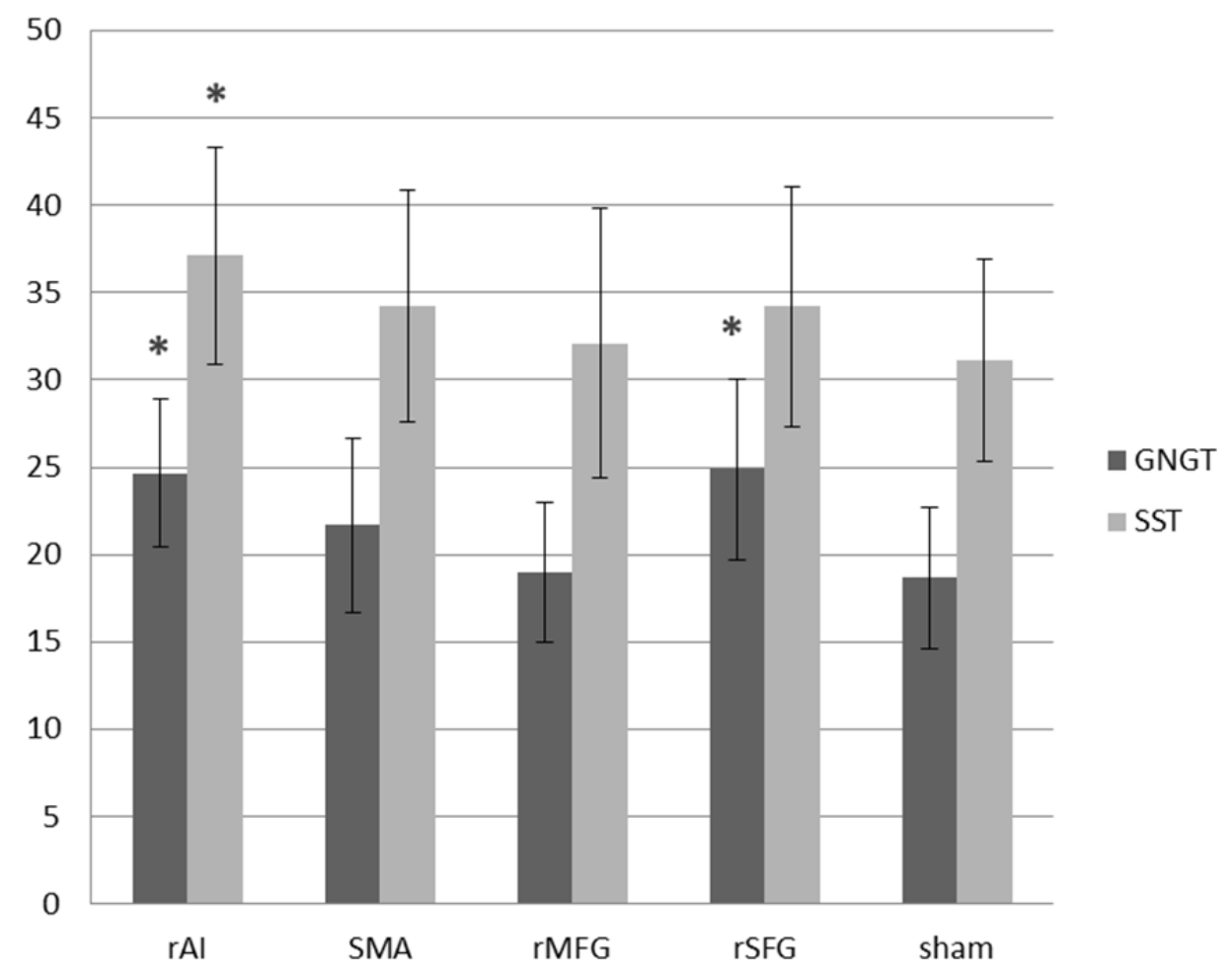

Fig.4. False alarm rates in inhibition trials for action restraint and action cancellation. Significant elevation of false alarm rates compared to the sham condition are labeled $\left({ }^{*} p>.05\right)$. Abbreviations: rAl right anterior insula, SMA pre-supplementary motor area, rMFG right middle frontal gyrus, rSFG right superior frontal gyrus, sham sham brain stimulation, GNGT go/no-go task, SST stop signal task.

Effects of stimulation on the go/no-go task. For action restraint the mixed model analysis revealed that compared to sham stimulation false alarm rates in inhibition trials were elevated following rAl stimulation (mean difference compared to sham condition=6.25, $=6.0, d f=32, p=.021$, Cohen's $d=0.59$ ) and rSFG stimulation (mean difference compared to sham condition=6.38, $F=6.24, d f=32, p=.019$, Cohen's $d=0.51$ ). No significant effects on false alarm rates were found for pre-SMA and rMFG stimulation (mean difference pre-SMA compared to sham condition=2.25, $F=.77, \mathrm{df}=32$, $p=.385$; mean difference $r M F G$ compared to sham condition=1.38, $F=.29, d f=32$, $\mathrm{p}=.594)$. An analysis of variance revealed no order effects with respect to the individual sessions for false alarm rates induced by for instance learning or habituation $(F(4,28)=.45, p=.769)$. Stimulation did not significantly affect response rates / misses ( $r A l: F=.04, d f=32, p=.843$; pre-SMA: $F=.42, d f=32, p=.521 ; r M F G: F=.06, d f=32$, $p=.805$; $r S F G: F=.09, d f=32, p=.767$ ) and reaction times ( $r A l: F=1.44, d f=32, p=.240 ; p r e-$ 
SMA: $F=.05, d f=32, p=.821$; $r M F G: F=.20, d f=32, p=.656$; $r S F G: F=2.09, d f=32, p=.158$ ) in go trials. Significant results are depicted in figure 4.

Effects of stimulation on the stop signal task. For action cancellation the mixed model analysis revealed that compared to sham stimulation false alarm rates in inhibition trials were elevated following rAl stimulation (mean difference compared to sham condition=6.13, $F=4.30, d f=31, p=.048$, Cohen's $d=0.32$ ). No significant effects on false alarm rates were found for pre-SMA, rMFG, and rSFG stimulation (mean difference pre-SMA compared to sham condition=3.75, $\mathrm{F}=1.0, \mathrm{df}=31, \mathrm{p}=.305$; mean difference rMFG compared to sham condition $=2.63, F=.31, d f=31, p=.582$; mean difference $r S F G$ compared to sham condition $=3.50, \mathrm{~F}=.67, \mathrm{df}=31, \mathrm{p}=.357$ ). An analysis of variance revealed no order effects with respect to the individual sessions for false alarm rates induced by for instance learning or habituation $(F(4,28)=1.29, p=.297)$. Stimulation did not significantly affect response rates / misses in go trials ( $\mathrm{rAl}: \mathrm{F}=.00, \mathrm{df}=31, \mathrm{p}=.995$; pre-SMA: $F=.07, d f=31, p=.786$; $r M F G: F=.30, d f=31, p=.584 ; r S F G: F=.48, d f=31$, $p=.493$ ) and stop signal reaction times (SSRT; $r A l: F=.01, d f=31, p=.908$; pre-SMA: $F=.16, d f=31, p=.690 ; r M F G: F=.00, d f=31, p=.939 ; r S F G: F=1.23, d f=31, p=.277$ ). After pre-SMA stimulation participants reacted significantly faster on go trials (mean difference compared to sham condition $=11.63 \mathrm{~ms}, \mathrm{~F}=4.51, \mathrm{df}=31, \mathrm{p}=.043$ ). Otherwise, reaction times ( $r A l: F=3.39, d f=31, p=.076$; $r M F G: F=.09, d f=31, p=.758$; $r S F G: F=2.43, d f=31$, $\mathrm{p}=.130$ ) in go trials have not been affected by stimulation. Significant results are depicted in figure 4 .

\section{DISCUSSION}

This study systematically investigated the role of right prefrontal cortex in controlled behaviour, more specifically in response inhibition. The disruption of neural activity in right inferior frontal regions as well as pre-SMA by means of cTBS was expected to corrupt the ability to generally inhibit responses. Furthermore, the disruption of rSFG activity was expected to impair action restraint (as measured with a GNGT) but not cancellation (as measured with a SST), while the disruption of rMFG was thought to impair action cancellation but not restraint. Results showed that inferior frontal regions are crucial for different aspects of inhibitory processing (action restraint and action cancellation), while superior frontal regions might be more relevant for action restraint as measured in go/no-go paradigms specifically. Stimulation applied to middle frontal regions and pre-SMA did not have any effect on inhibitory processing.

Right inferior frontal cortex. According to our expectations the disruption of neural activity in right inferior frontal cortex strongly affected inhibitory processing in general. CTBS to rAl impaired the ability to restrain responses (measured with a GNGT) as well as the ability to cancel reactions to a certain configuration of stimuli (measured with a SST). This is in line with a vast body of imaging literature emphasizing the crucial role of 
right inferior frontal cortex in inhibition (Aron et al., 2004). Right inferior frontal regions have been found to be activated during successful inhibition across different response inhibition modalities. Thereby, paradigms such as the stop signal (Boehler et al., 2010; Chambers et al., 2009; Duann et al., 2009; Swick et al., 2011), the go/no-go (Simmonds et al., 2008; Swick et al., 2011), and the anti-saccade task (Chikazoe et al., 2007) have been employed. Furthermore, all brain stimulation studies on response inhibition that included right inferior frontal regions as target sites demonstrated an effect of brain stimulation on inhibition even across different stimulation modalities (rTMS: Chambers et al., 2006, 2007; cTBS: Verbruggen et al., 2010; tDCS: Jacobson et al., 2011). Thus, our results further support the notion of right inferior frontal cortex being crucial for successful action inhibition and emphasize that this holds true even across different aspects of inhibitory processing employing a within-subject approach. However, there are two issues to consider when interpreting our findings:

So far, within the inferior frontal cortex specifically the inferior frontal gyrus has been subject of brain stimulation research. Our study is the first to not target rIFG, but rAl. The decision to target rAl was made with respect to our fMRI findings on which the localization of individual target regions was based. Although with sufficiently liberal significance level extending into rIFG, the most significant "hotspot" of inferior frontal activation during successful inhibition - for both action restraint and action cancellation - was found to be localized within the anterior insula. This was the case for analyses of action restraint and action cancellation independently on group and also single subject level in our data set. In their meta-analysis of 42 studies of GNGTs and SSTs, Swick and colleagues (2011) also found the most prominent overlap of inferior frontal activation to be in anterior insula. Sharp and colleagues (2010) labeled their focus of activation as "IFG / insula" and thereby acknowledged the role of anterior insula alongside IFG activation in response inhibition research. With respect to cTBS stimulation, however, one has to be aware of the fact that deeper brain structures cannot be targeted without also stimulating the more superficial regions lying on top. Therefore, we have to emphasize that by targeting their individual "hotspot "of activation during successful inhibition within anterior insula, for some participants the most anterior part of the inferior frontal gyrus (around BA13; see Fig.3) was also exposed to stimulation.

Furthermore, labeling the function of inferior frontal regions in response inhibition merely as "inhibition" has to be evaluated with caution. Hampshire and colleagues (2010) concluded from their imaging study that inferior frontal cortex might play a more attentional than inhibitory role in response inhibition. They argue that IFG is recruited as soon as important cues have to be processed, regardless of whether a motor response has to be generated or inhibited. However, our findings clearly show that performance on trials in which the generation of a motor response is requested (go trials) is not affected by the disruption of neural activity in inferior frontal regions, while the successful inhibition of a motor response (in no-go and stop trials) is. Ver- 
bruggen and colleagues (2010) further specified the effect on response inhibition. They showed that the disruption of neural tissue in specifically the ventral part of the right IFG by means of cTBS did affect both, stop signal trials (in which subjects had to withhold their response) and dual signal trials (in which subjects had to execute an additional response). The more dorsal part of the rIFG seemed more crucial for detecting changes in the visual environment in the first place. With respect to anterior insula function, involvement in saliency processing, switching of action plans, attention, and cognitive control (Vinod Menon \& Uddin, 2010) have been emphasized. Functionally, anterior insula can be described as the general control instance the cognitive system accesses whenever actions need to be changed; thereby, actions can range from simple motor responses as in the presented study to complex forms of social interaction (White, Brislin, Meffert, Sinclair, \& Blair, 2013).

Our findings clearly emphasize the crucial role of right inferior frontal cortex, and specifically right anterior insula in action restraint and action cancellation and, thus, these specific forms of controlled behaviour. It would be of interest to examine whether IFG and Al could be functionally discriminated in the context of inhibition research. This could cast further light on which specific functions - with respect to attention, updating and changing of action plans, attention, and stopping of actions inferior frontal cortex incorporates.

Right middle and superior frontal cortex. Based on our fMRI localizer (see Fig.2), we expected that cTBS applied to rSFG would impair action restraint but not cancellation, while cTBS applied to rMFG would impair action cancellation but not restraint. Our findings support the former hypothesis, while they do not support the latter.

Our findings do not suggest a crucial involvement of middle frontal cortex in response inhibition. In contrast, Zheng and colleagues (2008) found a correlation between intensity of neural activation in rMFG and performance in both a GNGT and a SST and, thus, identified the middle frontal cortex as the "key locus" of inhibitory control. Neither the fMRI localizer nor the brain stimulation data in our study support this notion. Although significantly activated during action cancellation (but not action restraint), cTBS targeting rMFG did not affect the ability to inhibit in either of the tasks. In response inhibition literature middle frontal cortex is seen ambiguously: Some imaging studies find its involvement in the GNGT and the SST (Zheng et al., 2008), some don't (Rubia et al., 2001), and some find only marginal involvement (McNab et al., 2008). The only brain stimulation study including a middle frontal area as a target site (Chambers et al., 2006) did not find any effect of rTMS applied to rMFG on inhibitory processing in a SST. Our findings extend these results as we replicate the absence of an effect of GTMS applied to MFG on the SST and in addition also find no effect on the GNGT. Although the absence of evidence is no evidence of absence (de Graaf \& Sack, 2011), the described converging evidence suggests that the role of rMFG in response inhibition is of a less substantial but rather supportive nature. Future studies could, therefore, a priori hypothesize such a lack of evidence regarding the causal role of 
rMFG in response inhibition and then apply Bayesian inferential analyses to directly test zero-findings and substantiate the assumption of an expected absence of evidence as evidence of absence (Verbruggen, Adams, et al., 2013).

In contrast, our results might suggest the notion of a crucial involvement of superior frontal activation, specifically in action restraint. Imaging studies reported the involvement of rSFG in GNGT related inhibition (Simmonds et al., 2008). However, also middle frontal brain regions have been found to be activated during successful inhibition in the GNGT (Swick et al., 2011). To our knowledge, so far neither MFG nor SFG have been investigated by means of brain stimulation in the context of action restraint (as measured in a GNGT). Our findings indicate that disruption of neural activity within rSFG might impair the ability to successfully inhibit responses in a GNGT, while rMFG stimulation does not. This suggests that the superior more than the middle frontal cortex is crucially involved in action restraint. Interestingly, TMS over rSFG did not affect the SST, indicating a potentially task-specific differential involvement of SFG in action restraint and not in action cancellation.

This is also in accordance with the imaging data acquired in the same sample. When directly contrasting fMRI activation during successful GNGT and SST inhibition, we found rSFG to be significantly involved in the GNGT, while rMFG was not. Therefore, based on our empirical fMRI data, we expected performance in the GNGT to worsen after rSFG but not rMFG stimulation. In response inhibition research there still seems to be no consensus on which specific middle and/or superior frontal cortical structures are crucial for inhibitory processing: Simmonds and colleagues (2008) report a superior frontal region (BA10) - similar to the one we find - and BA9 in their activation likelihood estimation over 11 studies investigating the GNGT. Swick and colleagues (2011) report as a result of their activation likelihood estimation over 21 studies investing the GNGT a middle frontal area (BA9) as the most crucial specific prefrontal area activated during the GNGT. Therefore, the role of middle and superior frontal areas for inhibition is still not clearly defined in fMRI literature and this motivated our closer look into the precise distribution of specific neural activity in prefrontal areas.

In summary, our results seem to imply that superior frontal cortex is involved in action restraint as measured by a GNGT but not in action cancellation as measured by a SST, while middle frontal cortex does not seem to be crucial in inhibitory processing. Of course, these findings are bound to the specific stimulations sites we chose within superior and middle frontal cortex (see Tab.1) based on individual functional imaging data. Furthermore, bearing in mind the rather small sample investigated in this study and the fact that effects have not been tested directly across tasks, our results regarding rSFG involvement in specific aspects of response inhibition should be interpreted with caution. In our analysis only a priori planned comparisons (differences in false alarm rates following stimulation of certain target sites compared to the sham condition) were tested. This does not enable strong claims regarding how TMS effects each site differently, but rather interpretations on how TMS affects each site compared to a 
condition in which no TMs is applied. Further studies are needed to establish and test theories on superior frontal involvement in response inhibition in order to enable stronger claims.

Pre-supplementary motor area. Contrary to our expectations we did not find any effect on inhibitory processing induced by disruption of neural activity within pre-SMA. In the SST participants even reacted slightly faster on go trials after pre-SMA stimulation compared to sham stimulation which should also induce more commission errors in inhibition trials. However, pre-SMA stimulation did not elevate false alarms rates in both tasks at all. This is in opposition to a vast body of imaging literature that emphasizes consistently the involvement of pre-SMA in motor response inhibition across different inhibitory domains including the GNGT and the SST (Swick et al., 2011). Furthermore, brain stimulation studies also reported effects of event related single pulse TMS (Chen et al., 2009) and tDCS (Hsu et al., 2011) applied to pre-SMA on inhibitory processing. However, it seems that these effects are difficult to replicate with repetitive TMS (Verbruggen et al., 2010). We explain our failure to reveal any behavioural effects on inhibitory processing for pre-SMA stimulation with the fact that the individual target sites for pre-SMA stimulation were located significantly deeper (mean coil cortex distance across all participants for pre-SMA 56mm) than for the other regions (mean coil cortex distance across all participants for rAl $50 \mathrm{~mm}$, for rMFG $33 \mathrm{~mm}$, for rSFG $34 \mathrm{~mm}$ ). Applying stimulation according to distance adjusted motor threshold (Stokes et al., 2007) could circumvent this problem. However, for the individual functional "hotspots" within pre-SMA we encountered, this would have exceeded common safety regulations by far (Rossi et al., 2009). In their recent work Obeso and colleagues (Obeso et al., 2013; Obeso, Robles, Marrón, \& Redolar-Ripoll, 2013) interfered successfully with pre-SMA activation using online as well as offline repetitive TMS and induced inhibitory failure. Furthermore, the authors find further evidence for the essential functional alliance of pre-SMA and inferior frontal areas in response inhibition as well as for the specific involvement of pre-SMA in the stopping rather than the switching of actions. Therefore, we conclude that cTBS as used in the current study is likely not an appropriate method to investigate pre-SMA function. Due to methodological choices and our rather small sample size, our zero result regarding pre-SMA can only be interpreted with caution.

\section{CONCLUSION AND IMPLICATIONS}

This study is the first to systematically investigate the differential involvement of several distinct functional sub-regions within right prefrontal cortex in different aspects of controlled behaviour. Our results emphasize the crucial role of inferior frontal cortex, specifically the right anterior insula, in action restraint (as measured with a GNGT) and action cancellation (as measured with a SST). Furthermore, superior frontal cortex 


\section{CHAPTER 3}

seems to be crucially involved in action restraint specifically, but not action cancellation. The role of middle frontal cortex in action inhibition seems to be of less substantial nature.

These findings enable a broader understanding of self-controlled behaviour assigned to humans and the neural mechanism underlying inhibitory failure. In how far results acquired in the context of simple motor response inhibition can be transferred to the rather complex inhibitory processing required in everyday social interactions remains an open question for further investigations. 
Aron, A. R., Robbins, T. W., \& Poldrack, R. A. (2004). Inhibition and the right inferior frontal cortex. Trends in Cognitive Sciences, 8(4), 170-7.

Band, G. P. H., van der Molen, M. W., \& Logan, G. D. (2003). Horse-race model simulations of the stop-signal procedure. Acta Psychologica, 112(2), 105-42.

Beeli, G., Casutt, G., Baumgartner, T., \& Jäncke, L. (2008). Modulating presence and impulsiveness by external stimulation of the brain. Behavioral and Brain Functions, 4, 33.

Boehler, C. N., Appelbaum, L. G., Krebs, R. M., Hopf, J. M., \& Woldorff, M. G. (2010). Pinning down response inhibition in the brain: Conjunction analyses of the Stop-signal task. Neurolmage, 52(4), 1621-32.

Chambers, C. D., Bellgrove, M. a, Stokes, M. G., Henderson, T. R., Garavan, H., Robertson, I. H., ... Mattingley, J. B. (2006). Executive "brake failure" following deactivation of human frontal lobe. Journal of Cognitive Neuroscience, 18(3), 444-55.

Chambers, C. D., Bellgrove, M. A., Gould, I. C., English, T., Garavan, H., McNaught, E., ... Mattingley, J. B. (2007). Dissociable mechanisms of cognitive control in prefrontal and premotor cortex. Journal of Neurophysiology, 98(6), 3638-47. d

Chambers, C. D., Garavan, H., \& Bellgrove, M. a. (2009). Insights into the neural basis of response inhibition from cognitive and clinical neuroscience. Neuroscience and Biobehavioral Reviews, 33(5), 631-46.

Chen, C.-Y., Muggleton, N. G., Tzeng, O. J. L., Hung, D. L., \& Juan, C.-H. (2009). Control of prepotent responses by the superior medial frontal cortex. Neurolmage, 44(2), 537-45.

Chikazoe, J., Konishi, S., Asari, T., Jimura, K., \& Miyashita, Y. (2007). Activation of right inferior frontal gyrus during response inhibition across response modalities. Journal of Cognitive Neuroscience, 19(1), 69-80.

De Graaf, T. A., \& Sack, A. T. (2011). Null results in TMS: from absence of evidence to evidence of absence. Neuroscience and Biobehavioral Reviews, 35(3), 871-7.

Duann, J.-R., Ide, J. S., Luo, X., \& Li, C. R. (2009). Functional connectivity delineates distinct roles of the inferior frontal cortex and presupplementary motor area in stop signal inhibition. The Journal of Neuroscience, 29(32), 10171-9.

Duecker, F., \& Sack, A. T. (2013). Pre-stimulus sham TMS facilitates target detection. PloS One, 8(3), e57765.

Faul, F., Erdfelder, E., Lang, A.-G., \& Buchner, A. (2007). G*Power 3: a flexible statistical power analysis program for the social, behavioral, and biomedical sciences. Behavior Research Methods, 39(2), 17591.

Hampshire, A., Chamberlain, S. R., Monti, M. M., Duncan, J., \& Owen, A. M. (2010). The role of the right inferior frontal gyrus: inhibition and attentional control. Neurolmage, 50(3), 1313-9.

Heatherton, T. F., \& Wagner, D. D. (2011). Cognitive neuroscience of self-regulation failure. Trends in Cognitive Sciences, 15(3), 132-9.

Hsu, T.-Y., Tseng, L.-Y., Yu, J.-X., Kuo, W.-J., Hung, D. L., Tzeng, O. J. L., ... Juan, C.-H. (2011). Modulating inhibitory control with direct current stimulation of the superior medial frontal cortex. Neurolmage, 56(4), 2249-57.

Huang, Y.-Z., Edwards, M. J., Rounis, E., Bhatia, K. P., \& Rothwell, J. C. (2005). Theta burst stimulation of the human motor cortex. Neuron, 45(2), 201-6.

Jacobson, L., Javitt, D. C., \& Lavidor, M. (2011). Activation of inhibition: diminishing impulsive behavior by direct current stimulation over the inferior frontal gyrus. Journal of Cognitive Neuroscience, 23(11), 3380-7.

Logan, G. D., Schachar, R. J., \& Tannock, R. (1997). Impulsivity and Inhibitory Control. Psychological Science, 8(1), 60-64.

McNab, F., Leroux, G., Strand, F., Thorell, L., Bergman, S., \& Klingberg, T. (2008). Common and unique components of inhibition and working memory: an fMRI, within-subjects investigation. Neuropsychologia, 46(11), 2668-82.

Menon, V., \& Uddin, L. Q. (2010). Saliency, switching, attention and control: a network model of insula function. Brain Structure \& Function, 214(5-6), 655-67.

Miller, E. K. (2000). The prefrontal cortex and cognitive control. Nature Reviews. Neuroscience, 1(1), $59-65$.

Muggleton, N. G., Chen, C.-Y., Tzeng, O. J. L., Hung, D. L., \& Juan, C.-H. (2010). Inhibitory control and the frontal eye fields. Journal of Cognitive Neuroscience, 22(12), 2804-12. 


\section{CHAPTER 3}

Obeso, I., Cho, S. S., Antonelli, F., Houle, S., Jahanshahi, M., Ko, J. H., \& Strafella, A. P. (2013). Stimulation of the pre-SMA influences cerebral blood flow in frontal areas involved with inhibitory control of action. Brain Stimulation, 6(5), 769-76.

Obeso, I., Robles, N., Marrón, E. M., \& Redolar-Ripoll, D. (2013). Dissociating the Role of the pre-SMA in Response Inhibition and Switching: A Combined Online and Offline TMS Approach. Frontiers in Human Neuroscience, 7, 150.

Rossi, S., Hallett, M., Rossini, P. M., \& Pascual-Leone, A. (2009). Safety, ethical considerations, and application guidelines for the use of transcranial magnetic stimulation in clinical practice and research. Clinical Neurophysiology, 120(12), 2008-39.

Rubia, K., Russell, T., Overmeyer, S., Brammer, M. J., Bullmore, E. T., Sharma, T., ... Taylor, E. (2001). Mapping motor inhibition: conjunctive brain activations across different versions of go/no-go and stop tasks. Neurolmage, 13(2), 250-61.

Sack, A. T., Cohen Kadosh, R., Schuhmann, T., Moerel, M., Walsh, V., \& Goebel, R. (2009). Optimizing functional accuracy of TMS in cognitive studies: a comparison of methods. Journal of Cognitive Neuroscience, 21(2), 207-21.

Sack, A. T., \& Linden, D. E. J. (2003). Combining transcranial magnetic stimulation and functional imaging in cognitive brain research: possibilities and limitations. Brain Research. Brain Research Reviews, 43(1), 41-56.

Sharp, D. J., Bonnelle, V., De Boissezon, X., Beckmann, C. F., James, S. G., Patel, M. C., \& Mehta, M. A. (2010). Distinct frontal systems for response inhibition, attentional capture, and error processing. National Academy of Sciences of the United States of America, 107(13), 6106-11.

Simmonds, D. J., Pekar, J. J., \& Mostofsky, S. H. (2008). Meta-analysis of Go/No-go tasks demonstrating that fMRI activation associated with response inhibition is task-dependent. Neuropsychologia, 46(1), 22432.

Stokes, M. G., Chambers, C. D., Gould, I. C., English, T., McNaught, E., McDonald, O., \& Mattingley, J. B. (2007). Distance-adjusted motor threshold for transcranial magnetic stimulation. Clinical Neurophysiology, 118(7), 1617-25.

Swick, D., Ashley, V., \& Turken, U. (2011). Are the neural correlates of stopping and not going identical? Quantitative meta-analysis of two response inhibition tasks. Neurolmage, 56(3), 1655-65.

Varnava, A., Stokes, M. G., \& Chambers, C. D. (2011). Reliability of the "observation of movement" method for determining motor threshold using transcranial magnetic stimulation. Journal of Neuroscience Methods, 201(2), 327-32.

Verbruggen, F., Adams, R. C., van 't Wout, F., Stevens, T., McLaren, I. P. L., \& Chambers, C. D. (2013). Are the effects of response inhibition on gambling long-lasting? PloS One, 8(7), e70155.

Verbruggen, F., Aron, A. R., Stevens, M. A., \& Chambers, C. D. (2010). Theta burst stimulation dissociates attention and action updating in human inferior frontal cortex. Academy of Sciences of the United States of America, 107(31), 13966-71.

Verbruggen, F., Chambers, C. D., \& Logan, G. D. (2013). Fictitious inhibitory differences: how skewness and slowing distort the estimation of stopping latencies. Psychological Science, 24(3), 352-62.

Verbruggen, F., \& Logan, G. D. (2009). Models of response inhibition in the stop-signal and stop-change paradigms. Neuroscience and Biobehavioral Reviews, 33(5), 647-61.

White, S. F., Brislin, S. J., Meffert, H., Sinclair, S., \& Blair, R. J. R. (2013). Callous-unemotional traits modulate the neural response associated with punishing another individual during social exchange: a preliminary investigation. Journal of Personality Disorders, 27(1), 99-112.

Zheng, D., Oka, T., Bokura, H., \& Yamaguchi, S. (2008). The key locus of common response inhibition network for no-go and stop signals. Journal of Cognitive Neuroscience, 20(8), 1434-42. 




\section{CHAPTER 4 Out of control}

\section{Evidence for anterior insula involvement in motor impulsivity and reactive aggression}

"Crime is terribly revealing. Try and vary your methods as you will, your tastes, your habits, your attitude of mind, and your soul is revealed by your actions."

- Agatha Christie, The ABC Murders, 1936 
Based on: Dambacher, F., Sack, A. T., Lobbestael, J., Arntz, A., Brugman, S., \& Schuhmann, T. (2014). Out of control Evidence for anterior insula involvement in motor impulsivity and reactive aggression. Social Cognitive and Affective Neuroscience, epub ahead of print.

We thank Ulrike Krämer, Frederike Beyer, Armin Heinecke, Martijn Stroom, Martijn van Teffelen, Clara Bernier, Oana Gurau, and Thomas C. Emmerling for their help. 


\section{ABSTRACT}

Inhibiting impulsive reactions while still defending one's vital resources is paramount to functional self-control and successful development in a social environment. However, this ability of successfully inhibiting, and thus controlling one's impulsivity, often fails, leading to consequences ranging from motor impulsivity to aggressive reactions following provocation. Although inhibitory failure represents the underlying mechanism, the neurocognition of social aggression and motor response inhibition have traditionally been investigated in separation. Here, we aimed to directly investigate and compare the neural mechanisms underlying the failure of inhibition across those different modalities of self-control. We employed functional imaging to reveal the overlap in neural correlates between failed motor response inhibition (measured by a go/no-go task) and reactive aggression (measured by the Taylor aggression paradigm) in healthy males. The core overlap of neural correlates was located in anterior insula, suggesting common anterior insula involvement in motor impulsivity as well as reactive aggression. This evidence regarding an overarching role of anterior insula across different modalities of self-control enables an integrative perspective on insula function and a better integration of cognitive, social, and emotional factors into a comprehensive model of impulsivity. Furthermore, it can eventually lead to a better understanding of clinical syndromes involving inhibitory deficits. 
In order to ensure survival and sufficient supply of necessary resources in a competitive world, impulsive reactions reverting to rather archaic mechanisms such as retaliation might sometimes be the right choice. At the same time, the ability to use one's cognitive capacities in order to inhibit overly impulsive actions seems equally important for the progressing of individuals within human societies. The right balance between impulsivity and inhibition is paramount to functional self-control and successful development in a social environment. However, this ability of successfully inhibiting, and thus controlling one's impulsivity, can also fail, leading to a variety of behavioural consequences ranging from poor motor response inhibition performance (motor impulsivity) to overly aggressive reactions to provocation in social interaction. Although for different behavioural outcomes the underlying mechanism might be a general failure of inhibition, the neurocognition of social aggression and motor response inhibition have traditionally been investigated in separation. The current study aims to directly investigate the overlap in neural components involved in motor impulsivity and reactive aggression.

Response inhibition - defined as the cognitive ability to deliberately withhold any planned or automatic reaction (Logan et al., 1997) -, as well as impulsive behaviour in the context of self-control, retaliation, and aggression, have attracted interest in cognitive and social neuroscience. Neuroimaging studies investigating the involvement of neural components in response inhibition mainly used simple motor response inhibition paradigms such as stop signal and go/no-go tasks (GNGTs). Such imaging studies consistently reported task-related activity within inferior frontal gyrus, anterior insula, pre-supplementary motor area, and subcortical circuitries involving thalamic regions and the striatum (Chambers et al., 2009; Simmonds et al., 2008; Swick et al., 2011). In most studies on inhibitory processing an asymmetric distribution of neuronal activity towards the right hemisphere was found (Aron et al., 2004).

The display of reactive aggression - defined as an aggressive reaction to provocation - has empirically been associated with impaired executive functioning (Hoaken et al., 2003). Looking at how response inhibition related neural activity might be mirrored in trait impulsiveness and self-reported reactive aggression might lead to important insights. Horn and colleagues (2003) showed that impulsive individuals (measured with Eysenck's Impulsivity Scale) reverted stronger right orbitofrontal activity in order to maintain inhibitory capacities in a GNGT. Furthermore, GNGT-inhibition related activity in the right dorsolateral prefrontal cortex was found to be negatively correlated with motor impulsiveness measured by the Barratt Impulsiveness Scale (Asahi et al., 2004). Recently, Pawliczek and colleagues (2013) showed that high trait aggressiveness (measured with a questionnaire) is attended not only by inhibition deficits in an emotional stop signal task on a behavioural level, but also lower inhibition related brain activity in pre-supplementary motor area and primary motor cortex. Despite their valuable achievement of explicitly demonstrating a link between aggression and different inhibition related brain regions that has mostly been built on implicit assumptions, 
the mentioned studies hold one constraint. The measures they used in order to determine impulsivity and aggression are self-report questionnaires. It has been demonstrated that such self-report measures correlate rather weakly with behavioural measures of similar concepts (Giancola \& Parrott, 2008). This is not surprising as both ways of measuring aggression refer to slightly different constructs. Therefore, it is rather interesting to apply neuroscientific methodology to both self-report measures and also actual behavioural measures. The latter has rarely been done. It is necessary to investigate common neural mechanism of motor impulsivity and aggression by looking at how behavioural inhibition measures such as the GNGT relate to other behavioural rather than self-report-based impulsivity or aggression measures.

When it comes to behavioural measurements of reactive aggression, the Taylor Aggression Paradigm (TAP; Taylor, 1967) proved itself the most feasible option in an imaging environment (Krämer et al., 2007; Lotze, Veit, Anders, \& Birbaumer, 2007). This task demonstrated high construct, internal, discriminant as well as external validity (C. a. Anderson, Lindsay, \& Bushman, 1999; Bernstein, Richardson, \& Hammock, 1987; Giancola \& Zeichner, 1995). During the task participants are provoked by one or more virtual opponent and their aggressive behaviour in reaction to provocation is measured by recording the severity level of the feedback or "punishment" they administer to their opponents. Punishment can be operationalized as administration of a highly aversive stimulus (e.g. an aversive noise or pneumatic pressure stimulus) to the opponent.

So far, surprisingly few imaging studies have investigated the neural correlates of reactive aggression in the TAP or a similar social interaction task in healthy adults (Krämer et al., 2007; Krämer, Kopyciok, Richter, Rodriguez-Fornells, \& Münte, 2011; Lotze et al., 2007), adolescents (White et al., 2013), and psychopaths (Veit et al., 2010). Most prominently anterior insula, anterior cingulate cortex, ventral and dorsal mediofrontal cortex, hypothalamic areas, and striatum were found to be involved in standard reactions to provocative situations and retaliation. Considering especially insula and the cingulate cortex activation, neural networks involved in response inhibition and reaction to provocation should substantially overlap. However, such an overlap has never been directly investigated with adequate methodology and, thus, claims on the specific and precise involvement of brain areas and their functional structure potentially contributing to several modalities of self-control remain to be tested.

Until today, no systematic inquiry evaluated the two concepts within the same psychological modality comparing a behavioural response inhibition paradigm such as the GNGT with a behavioural paradigm measuring aggression such as the TAP. The present study combines the advantages of employing both of these tasks with the high spatial resolution of functional Magnetic Resonance Imaging (fMRI) in a within-subject, within-session design. This enabled exploratory investigations in order to identify and compare network neural components involved in successful and unsuccessful response inhibition as well as aggressive reactions within provocative social interactions. 


\section{MATERIALS AND METHODS}

Participants. Eighteen male participants volunteered, gave their written informed consent and were paid for participating in this study. An extensive screening on neurological and psychological syndromes ensured that all participants were healthy. Only males were included to avoid gender related confounding effects. Two participants had to be excluded from the analyses, as they did not perform the tasks according to the instructions of the experimenter. One participant was excluded, as he did not show any reaction to provocation (he did not show any variance in his behaviour and only chose equally low reactions). Data of fifteen participants were included in further analyses $($ mean age $=22.33 ; \mathrm{SD}=2.35$ ). Most participants were university students and were recruited via advertisements on university related websites and flyers.

Procedure. Prior to the experiment, participants were told that they would take part in an experiment investigating the impact of human feedback on reaction time performance, together with two other participants. They were instructed that one task was performed (GTNT) independently and one task (TAP) would be a reaction time game in which all three participants would play together. Before entering the scanner, the participant and the two opponents (collaborators of the experimenters) were introduced, had a chance for casual conversation, and followed the experimenters' instruction together. The experimenter's collaborators were trained beforehand and acted according to a script in order to keep interactions constant with each participant. Throughout the experiment, the real participant would hear the experimenter talk with the "fake" opponents via intercom during the breaks. Participants performed a GNGT and the TAP. Task-order was counterbalanced. Immediately after completion of the experiment, participants underwent an oral manipulation check in order to make sure that they were fully deceived by the experimental setup. After all the measurements had been finalized, they were provided with a written debriefing in order to explain the deception and disclose the experiments' real purpose and motivation. The study was approved by the local Ethical Committee of the Faculty of Psychology and Neuroscience at Maastricht University and conformed to the Code of Ethics of the World Medical Association (Declaration of Helsinki).

Go/no-go task (GNGT). In order to elicit action restraint, a simple go/no-go motor response task was used (Fig.1A). Participants were instructed to respond as fast and accurately as possible to a go stimulus via button press with the right index finger, while they should not respond to a rare no-go stimulus. Go as well as no-go stimuli were presented for $200 \mathrm{msec}$ followed by a fixation cross for a randomized interval of 1300,2800 , or 4300 msec. No-go events occurred in $25 \%$ of all trials. The trials were pseudo-randomized in order to avoid unreasonably long concatenations of only inhibition events.

Stimuli and fixation crosses were presented in white (RGB 255/255/255; Arial pt 24) on a grey background (RGB 125/125/125). Participants performed two runs of 200 
trials each for the GNGT, leading to a total of 400 trials (100 no-go trials). Stimuli were presented using Presentation software (Neurobehavioural Systems, Inc., Albany, USA). Behavioural statistical analyses were performed using SPSS19 (IBM Statistics, USA).

A square and a circle were used as stimuli. Which stimulus was assigned to the go respectively the no-go condition was randomized between participants. After every 40 trials there was a resting period of 19500 msec integrated into the task, where only a fixation cross was shown.

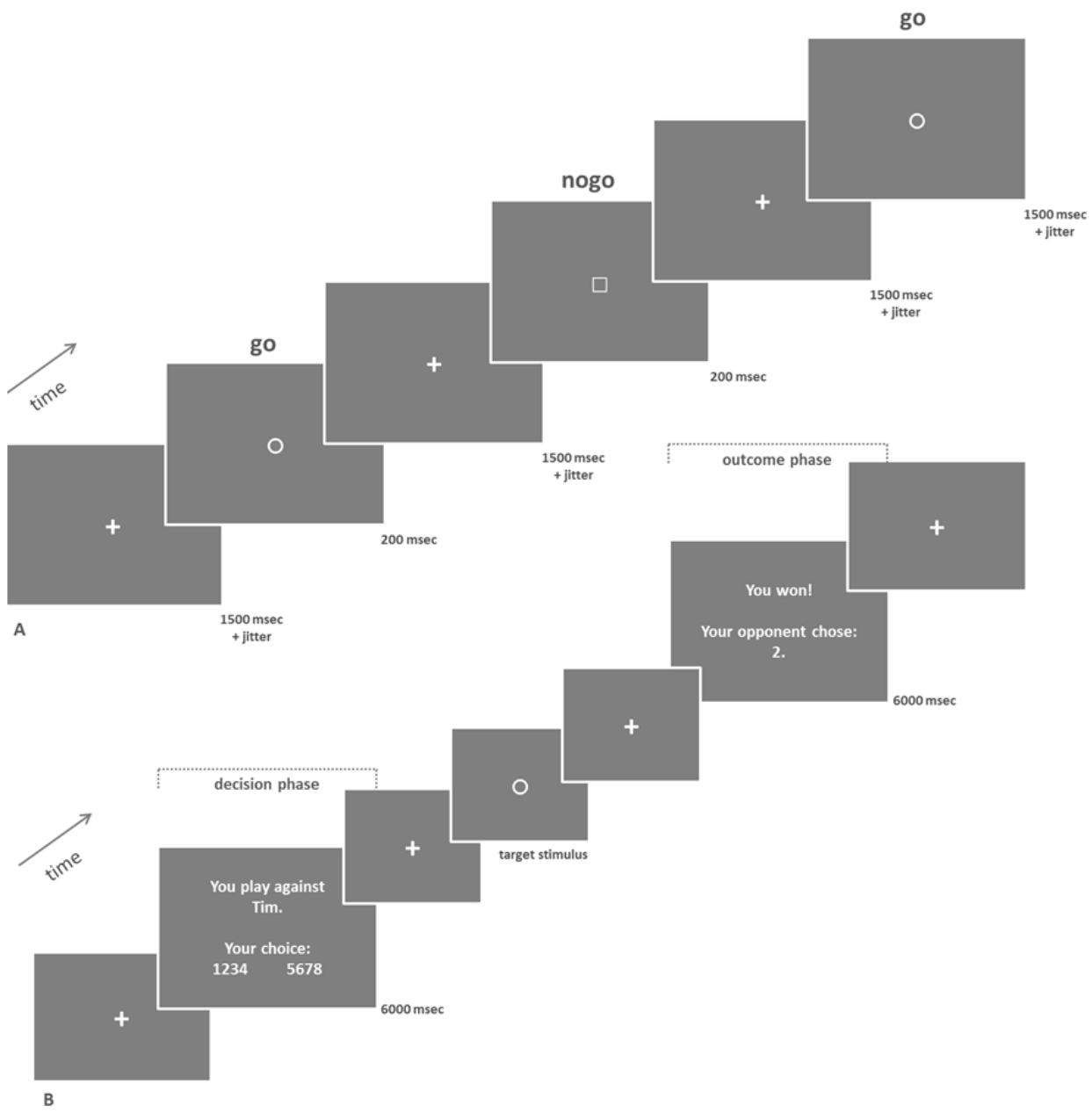

Fig.1. Go/no-go task (GNGT, A) and Taylor aggression paradigm (TAP, B). During the decision phase, participants were presented with a screen that informed them against whom they were playing in this trial (in this case "Tim") and asked to choose the feedback noise level that should be administered to this opponent in case the opponent would lose ("1234 5678"). During the outcome phase, participants were informed on whether or not they won and what feedback noise level the particular opponent chose for this trial. 
Taylor Aggression Paradigm (TAP). In order to elicit reactive aggression, an adaptation of the Taylor aggression paradigm (Krämer et al., 2007; Taylor, 1967) was used (Fig.1B). Participants were told that they would play a competitive reaction time game against two opponents. Whoever would be slower in reacting to a target stimulus by button press with the right index finger, would lose the trial and be administered an aversive feedback noise chosen by the winning opponent. The intensity of the feedback noises could be set by the participant for each trial on a scale from 1 (lowest noise) to 8 (loudest noise). For analyses, the feedback given by the participants was grouped into low (level 1-3), middle (4 and 5), and high (level 6-8) punishment. All task parameters were controlled by the experimenters as follows: Participants randomly played against each of the putative opponents in $50 \%$ of the trials. One of the opponents always selected relatively low feedback noises (from 1 to 4; non-provoking opponent) and the other relatively loud feedback noises (from 4 to 8; provoking opponent). Participants randomly won (and lost) in $50 \%$ of trials for each opponent with the constraint that if they needed longer than 500 msec to respond, they always lost. This ensured the credibility of competing against a human opponent.

Each trial of $27000 \mathrm{msec}$ consisted of a decision phase (6000 msec), the actual reaction time game (jittered between 4500 and $7500 \mathrm{msec}$ ), an outcome phase (6000 $\mathrm{msec}$ ), and a jittered resting period. During the decision phase, participants were presented with a screen that informed them against whom they were playing in this particular trial ("Rob" or "Tim") and asked to choose the feedback noise level that should be administered to this opponent in case he would lose. As the actual reactive aggressive behaviour is measured during the decision phase of the TAP, the analyses focused exclusively on this phase. During the outcome phase, participants were informed about whether or not they won or lost in this particular trial and what feedback noise level the particular opponent chose for this trial. In case of losing, the feedback noise was administered right after the outcome information was presented.

Stimuli and fixation crosses were presented in white (RGB 255/255/255; Arial pt 24) on a grey background (RGB 125/125/125). Participants performed 3 runs of the TAP including 28 trials ( 14 trials per opponent) each, leading to a total of 84 trials ( 42 trials per opponent). Stimuli were presented using Presentation software (Neurobehavioural Systems, Inc., Albany, USA). Behavioural statistical analyses were performed using SPSS19 (IBM Statistics, USA).

Feedback noises were adjusted to a subjective threshold of endurability while running a functional sequence before the experiment started. This way it was possible to control for scanner noise. No noises above 100 decibel were administered in order to ensure that the hearing of the participants could be in no way compromised.

Technical details, fMRI acquisition, and analysis. Stimulus material was displayed onto a frosted screen, positioned at rear of the scanner bore, using an LCD projector (Panasonic, No PT-EZ57OEL). Responses were collected employing a standard MR 
compatible button box (Current Designs, 8-button response device, HHSC-2x4-C, Philadelphia, USA).

Using a 3 Tesla Siemens Prisma MR scanner structural (high resolution T1-weighted MPRAGE; isotropic voxel resolution $1 \times 1 \times 1 \mathrm{~mm}^{3} ; 192$ sagittal slices) and functional whole-brain (Gradient-Echo-EPI-sequence; TR=1500msec; TE $=26 \mathrm{msec}$; $F O V=224 \mathrm{~mm}$; flip angle $=73^{\circ}$; matrix $=64 \times 64$; distance factor $=20 \%$; 478 volumes per run for the GNGT, 512 volumes per run for the TAP) images were acquired. 28 oblique transversal slices of $3.5 \times 3.5 \times 3.5 \mathrm{~mm}$ voxel tilted $30^{\circ}$ relatively to the anterior-posterior commissure plane were obtained in order to avoid signal dropout in frontal areas (Deichmann et al., 2003).

Data analyses were performed using Brain Voyager QX (Brain Innovation BV, Maastricht, The Netherlands). Prep-scans at the beginning of each functional run ensured equilibrium effects for signal saturation. Preprocessing included 3D-motion-correction (as implemented in Brain Voyager QX with trilinear / sinc interpolation and intrasession alignment to the 1st functional volume recorded after the individual anatomical scan), cubic spline slice scan time correction, and the application of a temporal high pass filter (general linear model (GLM) with Fourier basis set of 3 cycles sine/cosine per run including linear trend removal). Images were co-registered to the individual anatomical scans and normalization to Talairach stereotaxic space (Talairach \& Tournoux, 1988). Volume time courses were spatially smoothed using a $6 \mathrm{~mm}$ full width half maximum Gaussian kernel.

In order to establish activation patterns for the two tasks, random effects group analyses were performed. A GLM was defined to analyze specific activation patterns for no-go trials and false alarms in the GNGT and specific activation patterns with respect to the behaviour displayed during the decision phase in the TAP. In the TAP, the first three trials per opponent were excluded from the analyses to ensure that analyses were based on the trials during which the participant could already estimate the behavioural pattern of the opponents. The following conditions were included as predictors a) for the GNGT: successful go trials, successful no-go trials, false alarms (commission errors on the no-go trials) and b) for the TAP: number of trials that participant choses high punishment for the provoking opponent, participant choses low punishment for the non-provoking opponent. Most participants rarely or never chose a low or middle punishment for the provoking opponent. Furthermore, a middle or high punishment for the non-provoking opponent was almost never chosen. Therefore, these conditions could not be taken into account on the level of group analyses. The following specific contrasts were examined: For the GNGT neural correlates of successful inhibition were investigated by contrasting successful no-go trials with successful go trials (no-go trials > go trials) and neural correlates of unsuccessful inhibition were investigated by contrasting unsuccessful no-go trials with successful go trials (false alarms > go trials). For the TAP neural mechanisms underlying aggressive reaction to the provoking opponent were examined by contrasting trials in which the participant 


\section{CHAPTER 4}

gave a high punishment to the provoking opponent with trials in which the participant gave a low punishment to the non-provoking opponent (provocation > no provocation). Furthermore, the overlap of neural activation during unsuccessful inhibition and aggressive reaction to the provoking opponent was revealed through a conjunction analysis (false alarms $>$ go trials ${ }^{\wedge}$ provocation $>$ no provocation).

To reduce error variance, one noise regressor consisting of the first eigenvariate time series from cerebrospinal fluid regions and motion artefacts were included into the analyses as covariates. Statistical maps were created using a threshold of $p<.001$ corrected for multiple comparisons by means of cluster threshold level estimation (1000 Monte Carlo simulation iterations; Forman et al., 1995).

\section{RESULTS}

\section{Behavioural data}

GNGT. On average, in the GNGT participants showed reaction times on go trials of $404.42 \mathrm{msec}$ ( $\mathrm{SD}=35.29$ ), and committed $1.58 \%$ misses (omission errors on go trials, $\mathrm{SD}=2.19$ ) as well as $24.4 \%$ false alarms (commission errors on no-go trials, $\mathrm{SD}=$ 12.36).

TAP. The average feedback (i.e. punishment by mean of auditory feedback noise) selected by the participants for the opponents was of medium intensity (MEAN = 3.54; $S D=.04)$. However, for the provoking opponent significantly higher feedback was chosen than for the non-provoking opponent (provoking opponent: $\mathrm{MEAN}=4.52, \mathrm{SD}=$ .64; non-provoking opponent: $\mathrm{MEAN}=2.56, \mathrm{SD}=1.17 ; \mathrm{t}=4.59, \mathrm{p}=<.001$ ). Within the 42 trials playing against the provoking opponent, participants selected averagely 22 times a high feedback $(6,7$, or 8 ; SD $=7.73)$, 9 times a middle feedback (4 or 5; SD = $5.80)$, and 11 times a low feedback $(1,2$, or 3 ; SD $=6.62)$. In contrast, for the nonprovoking opponent a reversed behavioural pattern was observed: participants now selected on average 6 times a high feedback $(6,7$, or 8 ; SD $=6.98), 6$ times a middle feedback ( 4 or 5 ; SD = 5.84), and 30 times a low feedback $(1,2$, or 3 ; SD $=11.48$ ). The mean reaction time to the target stimulus was $204.53 \mathrm{msec}(\mathrm{SD}=31.83)$. During the oral manipulation check no participant doubted the cover story for the experiment and all fifteen participants reported that they perceived one opponent as more provocative than the other. Twelve participants reported explicitly that they adapted their reaction to that perception. Similarly, an analysis of variance with repeated measures revealed a significant interaction effect of high, middle, and low feedback level chosen by the participants and the type of opponent $(F=19.5, p<.001)$. Thereby, high punishment was chosen more frequently for the provoking than the non-provoking opponent, low punishment was chosen more often for the non-provoking than for the provoking opponent. 
FMRI data. Talairach coordinates of the brain regions showing increased activation associated with the investigated contrasts are reported in table 1. Statistical maps of random effects group analyses are depicted in figure 2 (for successful inhibition) and 3 (for unsuccessful inhibition, aggressive reaction to provocation, and the corresponding activation overlap).

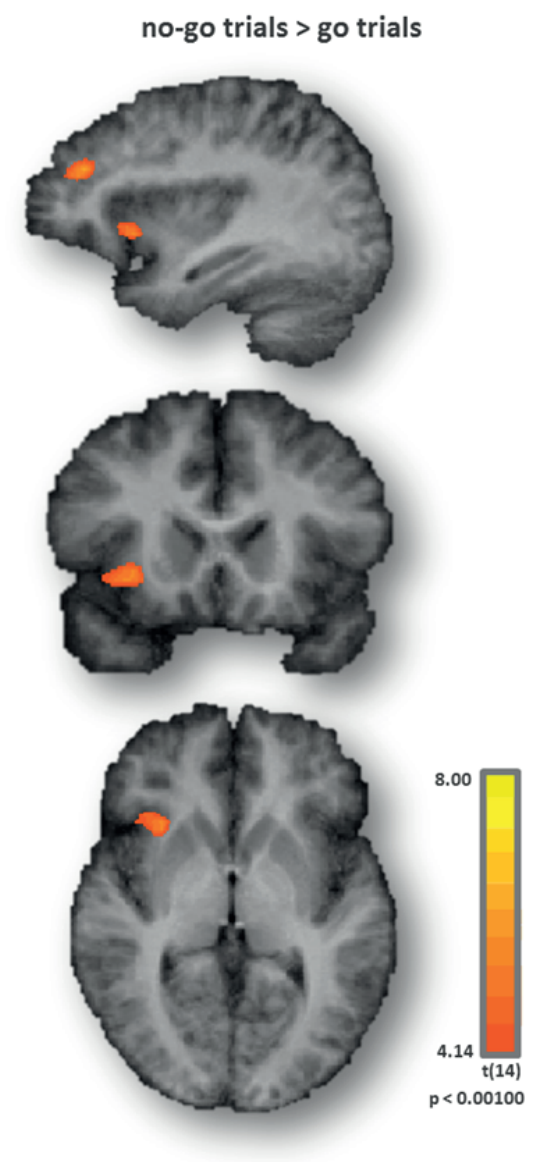

Fig.2. Successful inhibition. Increased cortical activation associated with successful inhibition during nogo trials vs. go trials (no-go trials > go trials; plane $\mathrm{x}(33) \mathrm{y}(18) \mathrm{z}(3))$. Statistical map $p \leq .001$; cluster level threshold (CLT) corrected; Random Effects group Analysis (RFX); $N=15$. Maps projected onto averaged anatomical scan of all 15 participants; radiological convention.

Table 1. Talairach coordinates of activated brain regions.

\begin{tabular}{|c|c|c|c|c|c|c|}
\hline \multirow[b]{2}{*}{ Region } & & \multicolumn{3}{|c|}{ Talairach coordinates } & \multirow{2}{*}{$\frac{\text { Size }}{\text { voxel }}$} & \multirow[b]{2}{*}{$t$} \\
\hline & & $x$ & $y$ & $z$ & & \\
\hline \multicolumn{7}{|l|}{ No-go trials > go trials } \\
\hline \multicolumn{7}{|l|}{ Frontal cortex } \\
\hline Anterior insula cortex & $\mathrm{R}$ & 31 & 17 & 1 & 596 & 6.12 \\
\hline Superior frontal gyrus & $\mathrm{R}$ & 35 & 38 & 26 & 688 & 7.34 \\
\hline \multicolumn{7}{|l|}{ Temporal/occipital cortex } \\
\hline Fusiform gyrus & L & -42 & -52 & -12 & 645 & 5.47 \\
\hline \multicolumn{7}{|l|}{ False alarms $>$ go trials } \\
\hline Frontal cortex & & & & & & \\
\hline
\end{tabular}




\begin{tabular}{|c|c|c|c|c|c|c|}
\hline \multirow[b]{2}{*}{ Region } & & \multicolumn{3}{|c|}{ Talairach coordinates } & \multirow{2}{*}{$\frac{\text { Size }}{\text { voxel }}$} & \multirow[b]{2}{*}{$t$} \\
\hline & & $x$ & $y$ & $z$ & & \\
\hline Insula cortex & $\mathrm{R}$ & 38 & 9 & 6 & 14153 & 9.11 \\
\hline Insula cortex & $\mathrm{L}$ & -43 & 5 & 6 & 30260 & 11.27 \\
\hline Middle frontal gyrus & $\mathrm{R}$ & 23 & 38 & 36 & 2343 & 6.44 \\
\hline Middle frontal gyrus & $\mathrm{L}$ & -25 & 38 & 36 & 5068 & 6.74 \\
\hline Superior frontal gyrus & $\mathrm{R}$ & 8 & -7 & 65 & 2471 & 6.33 \\
\hline Superior frontal gyrus & $\mathrm{L}$ & -14 & -12 & 65 & 4018 & 6.61 \\
\hline \multicolumn{7}{|l|}{ Cingulate cortex } \\
\hline Anterior cingulate cortex & & 0 & 11 & 37 & 22392 & 8.58 \\
\hline \multicolumn{7}{|l|}{ Parietal cortex } \\
\hline Supramarginal gyrus & $\mathrm{R}$ & 51 & -46 & 32 & 1411 & 5.67 \\
\hline Supramarginal gyrus & $\mathrm{L}$ & -54 & -46 & 32 & 8080 & 7.12 \\
\hline \multicolumn{7}{|l|}{ Occipital cortex } \\
\hline Lingual gyrus & $\mathrm{R}$ & 11 & -65 & 3 & 27854 & 6.88 \\
\hline Lingual gyrus & $\mathrm{L}$ & -18 & -64 & 0 & $(R \& L)$ & $(\mathrm{R} \& \mathrm{~L})$ \\
\hline \multicolumn{7}{|l|}{ Subcortical regions } \\
\hline Thalamus & $\mathrm{R}$ & 10 & -12 & 8 & 1016 & 5.84 \\
\hline Thalamus & $\mathrm{L}$ & -15 & -14 & 8 & 7972 & 5.66 \\
\hline \multicolumn{7}{|l|}{ Provocation>no provocation } \\
\hline \multicolumn{7}{|l|}{ Frontal cortex } \\
\hline Anterior insula cortex & $\mathrm{R}$ & 27 & 17 & 6 & 718 & 6.11 \\
\hline Anterior insula cortex & $\mathrm{L}$ & -33 & 18 & 16 & 1104 & 5.43 \\
\hline Insula cortex & $\mathrm{L}$ & -41 & -7 & 18 & 769 & 6.46 \\
\hline \multicolumn{7}{|l|}{ Parietal cortex } \\
\hline Inferior parietal lobe & $\mathrm{L}$ & -41 & -35 & 50 & 19896 & 8.58 \\
\hline \multicolumn{7}{|l|}{ Subcortical regions } \\
\hline Putamen / globus pallidus & $\mathrm{R}$ & 13 & 3 & 5 & 1307 & 6.58 \\
\hline Putamen / globus pallidus & $\mathrm{L}$ & -15 & 0 & 5 & 1098 & 6.06 \\
\hline Thalamus & $\mathrm{L}$ & -19 & -19 & 8 & 1104 & 5.37 \\
\hline Caudate & $\mathrm{L}$ & -17 & -8 & 24 & 601 & 5.82 \\
\hline \multicolumn{7}{|l|}{ Cerebellum } \\
\hline Anterior lobe & $\mathrm{R}$ & 13 & -48 & -18 & 800 & 5.24 \\
\hline Posterior lobe & $\mathrm{R}$ & 15 & -74 & -16 & 2277 & 7.15 \\
\hline \multicolumn{7}{|c|}{ False alarms > go conjunction with } \\
\hline \multicolumn{7}{|l|}{ Provocation > no provocation } \\
\hline \multicolumn{7}{|l|}{ Frontal cortex } \\
\hline Anterior insula cortex & $\mathrm{R}$ & 29 & 24 & 9 & 358 & 5.59 \\
\hline Anterior insula cortex & $\mathrm{L}$ & -35 & 16 & 12 & 1075 & 5.43 \\
\hline Insula cortex & $\mathrm{L}$ & -45 & -4 & 14 & 416 & 5.29 \\
\hline \multicolumn{7}{|l|}{ Subcortical regions } \\
\hline Thalamus & $\mathrm{L}$ & -17 & -18 & 6 & 395 & 5.06 \\
\hline Putamen / globus pallidus & $\mathrm{L}$ & -15 & -2 & 7 & 124 & 4.98 \\
\hline
\end{tabular}

For successful inhibition (no-go trials > go trials), the examination of blood oxygenation level dependent signal change in 15 participants, revealed a significant increase of activation in right anterior insula cortex (AIC), right superior frontal gyrus, and left fusiform gyrus (Fig.2, Tab.1). 
During unsuccessful inhibition (false alarms > go trials) associated with commission errors in the inhibition trials increased activation in bilateral insula cortex, bilateral middle and superior frontal gyri, anterior cingulate cortex (ACC), and thalamus was found. Furthermore, the supramarginal gyri and occipital regions showed significantly increased activation for unsuccessful inhibition in the GNGT (Fig.3, Tab.1). When contrasting trials in which the participant gave a high punishment to the provoking opponent with trials in which the participant gave a low punishment to the non-provoking opponent (provocation > no provocation), increased activation in bilateral insula cortex, inferior parietal lobe, and cerebellum was observed. Furthermore, several subcortical regions (i.e., the right and left putamen/globus pallidus, left-lateralized thalamic regions and caudate) showed significant activation change associated to this contrast (Fig.3, Tab.1). No significant activation change associated with giving a low punishment to the non-provoking opponent (no provocation > provocation) was observed.

When examining the significant overlap of neural activation associated with unsuccessful inhibition and aggressive reaction to provocation (false alarms $>$ go trials $\wedge$ provocation > no provocation), bilateral AIC, extended parts of the insula cortex in the left hemisphere, as well as left-lateralized subcortical structures (i.e., thalamus and putamen/globus pallidus) showed to be activated for both aspects of dis-inhibition likewise (Fig.3, Tab.1). Furthermore, activation in right AIC during unsuccessful inhibition positively correlated with activation in right AIC during the condition in which a high punishment was assigned to the provoking opponent (right AIC: $r=.642, p=.01$; correlations were obtained by calculating bivariate Pearson correlations between betaweights for both conditions across participants extracted from a region of interest defined by activation in right AIC). No correlations were found for the other regions showing maximal overlap of activation in the conjunction analysis (left AIC: $r=.386, p=$ .156; subcortical structures: $r=.408, p=.131$; correlations were obtained by calculating bivariate Pearson correlations between beta-weights for both conditions across participants extracted from a region of interest defined by activation in left AIC or a common region of interest including all relevant subcortical structures). 


\section{A) false alarms $>$ go trials}
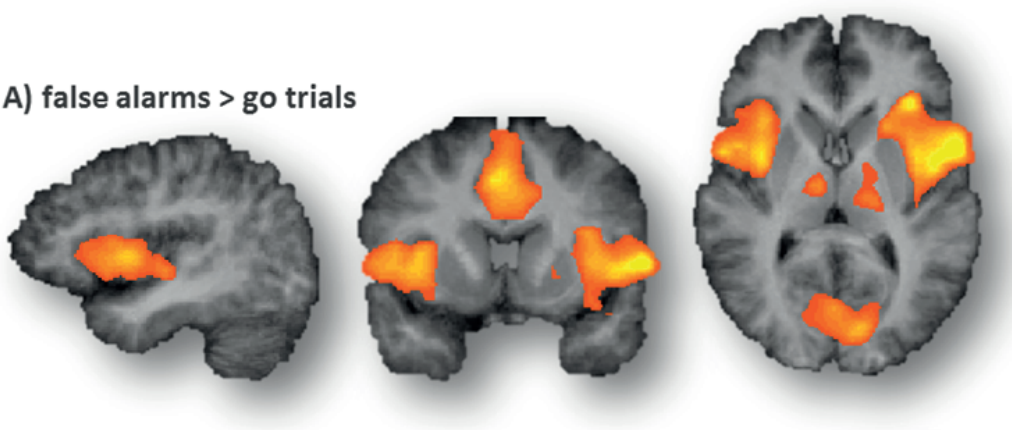

\section{B) provocation > no provocation}
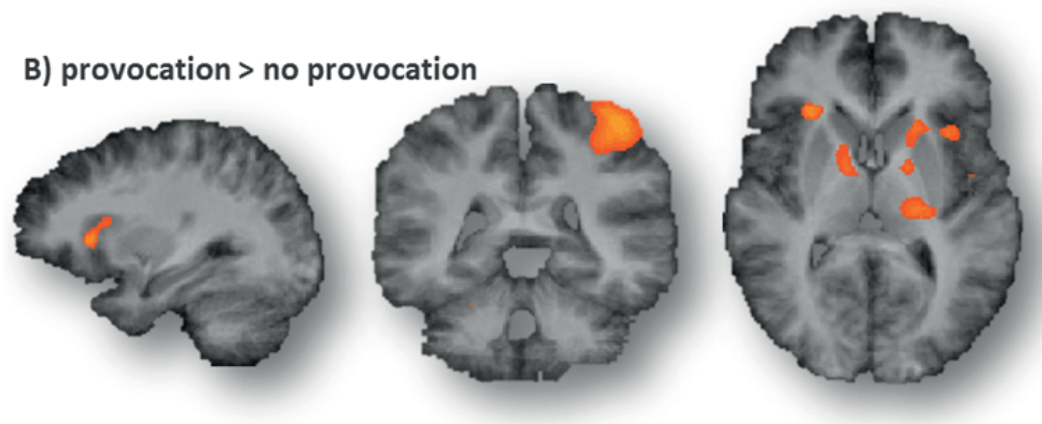

\section{C) false alarms $>$ go trials $\Lambda$ provocation > no provocation}
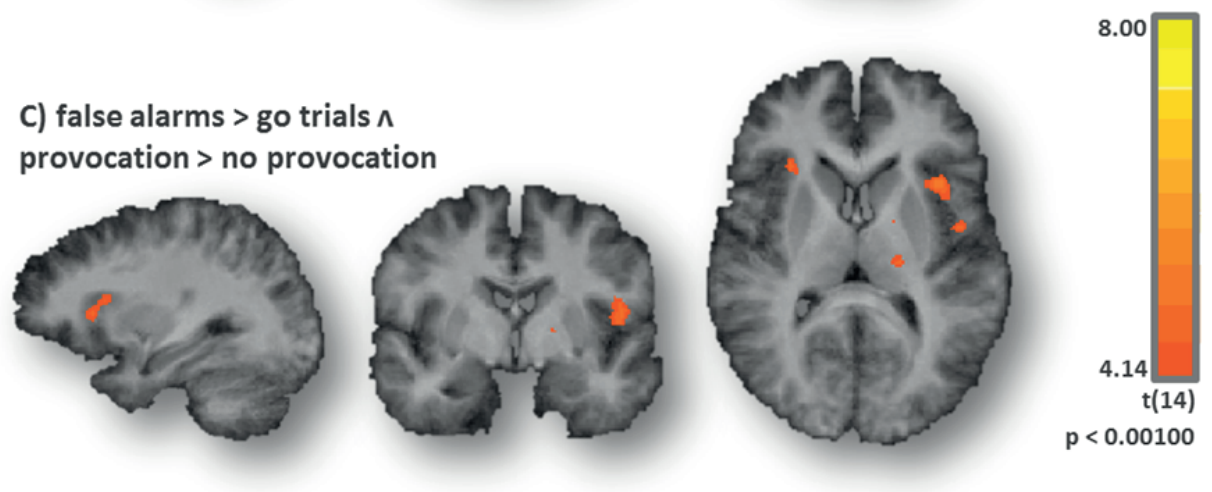

Fig. 3. GLM maps. A) Increased cortical activation associated with unsuccessful inhibition during no-go trials vs. go trials (false alarms > go trials; plane $x(37) y(9) z(8)$ ). B) Increased cortical activation associated with aggressive reaction to provoking opponent vs. non-aggressive reaction to non-provoking opponent (provocation > no provocation; plane $x(28) y(-37) z(8))$. C) Increased cortical activation overlapping for aggressive reaction to provocation and unsuccessful inhibition during no-go trials (false alarms $>$ go trials ${ }^{\wedge}$ provocation > no provocation; plane $\mathrm{x}(28) \mathrm{y}(-4) \mathrm{z}(10))$.Statistical maps $p \leq .001$; cluster level threshold (CLT) corrected; Random Effects group Analysis (RFX); $N=15$. Maps projected onto averaged anatomical scan of all 15 participants; radiological convention. 


\section{DISCUSSION}

We employed two behavioural paradigms (GNGT, TAP) to investigate the neural components involved in successful and unsuccessful response inhibition as well as aggressive reactions in provocative social interactions. We also examined in how far neural activation associated with unsuccessful inhibition and aggressive reaction to provocation overlaps.

Response inhibition. We found right AIC and right superior frontal gyrus to be activated during successful response inhibition as measured by a simple go/no-go motor response task. These findings are in accordance with previous literature on motor action inhibition (Simmonds et al., 2008; Swick et al., 2011). We found a rightlateralization of inhibition related activation, which has been reported to be associated to various inhibition paradigms before (Aron et al., 2004; Chambers et al., 2009; Swick et al., 2011). Evidence is growing that besides right inferior frontal gyrus (Aron et al., 2004; Chikazoe et al., 2007), AIC plays a crucial role in response inhibition across different aspects of inhibition (Sharp et al., 2010; Swick et al., 2011). In their activation likelihood estimation over 11 studies investigating the GNGT, Simmonds and colleagues (2008) report AIC to be involved specifically in complex go/no-go paradigms. However, in our paradigm, which would be defined as a simple GNGT according to Simmonds' criteria, we find the same activation. Repeatedly, superior or middle fontal regions similar to the one we find in our analyses have been reported to be crucially involved in GNGT performance (Simmonds et al., 2008; Swick et al., 2011).

During unsuccessful inhibition we found a significant activation pattern across many cortical and subcortical areas. It should be emphasized that right AIC and right superior frontal gyrus - the two regions that were specifically involved in successful inhibition - are part of the activation pattern of unsuccessful inhibition. Menon and colleagues (2001) found a pattern similar to ours and acknowledged the substantial overlap of error related brain activation with activation found during successful inhibition in a GNGT. They also found a more distributed network for unsuccessful than for successful inhibition and emphasized that this is in accordance with ideas resulting from previous EEG studies. The involvement of AIC in successful as well as unsuccessful inhibition might also reflect dynamic behavioural control across different modalities of cognition rather than response inhibition specifically.

Furthermore, the current finding might be explained by a post-error overshoot of the neural network crucial for the execution of a certain task. This would correspond to the subjective feeling of error awareness or mental overcompensation - expressed by elevated alertness, alarm, and intense visualization of what should have been done - following an inaccurate reaction (Klein et al., 2007). Furthermore, especially regions such as ACC and AIC that have repeatedly been associated with the successful execution of cognitive tasks, have also been reported to be crucially involved in momentary lapses of attention (Weissman, Roberts, Visscher, \& Woldorff, 2006) and maladaptive 
changes of event related brain networks (Eichele et al., 2008). Along these lines it should be emphasized that, when errors occur, the participant has to deal with a subjective feeling of failing or blundering which is not necessarily present in an aggressive reaction to provocation. This might be an explanation of our finding that the ACC is specifically involved in motor impulsivity but not the TAP.

Generally, it is debatable in how far specificity of functional involvement with respect to certain brain regions can be assumed in this context. The mentioned brain areas might rather be involved in general cognitive control and monitoring of actions than in the specific cognitive aspect a task is supposed to tap into. The activations could not only be related to the inability to withhold a response, but also to error monitoring and reactions to committing an error and subsequently implementing a cognitive control process. Overall activations might be due to more than just greater impulsivity.

Additionally, it is crucial to emphasize that we cannot rule out emotional processes that came along with error processing. False alarms, thus not succeeding in the specific task, could be accompanied by a sense of failure and subsequently negative emotions such as irritation and anger. Therefore, related neural activity might also reflect such emotional processes.

Aggressive reaction to provocation. In order to elicit aggressive reactions in a provocative social interaction we employed the Taylor aggression paradigm. Participants consistently choose significantly higher feedback, which means more uncomfortable noise stimuli, for the provoking compared to the non-provoking opponent and afterwards reported to deliberately have done so. Not just qualitatively, but also quantitatively we could confirm that the behavioural pattern varied with respect to the characteristics of the opponent. This implies that our experimental manipulation was successful and that we managed to simulate interactions as similar to natural behaviour as possible in a laboratory situation. However, at the same time this very pronounced and natural behavioural pattern restricted our analyses to the two most obvious reactions: aggressive reactions to provocation and no aggressive behaviour following no provocation. Therefore, in our data the intensity of provocation and the level of the chosen punishment cannot be disentangled. Ultimately though, this is the case for any retaliating behaviour observable in a natural environment and, thus, provocation and the resulting retaliation is understood as a unity in the presented analyses. When directly contrasting these two conditions, no significant change of neural activation specifically associated with a not aggressive reaction to the non-provoking opponent was observed.

During periods in which participants reacted aggressively towards the provoking opponent increased neural activation was found in bilateral insula cortex, left inferior parietal lobe, a variety of mostly left lateralized subcortical structures (i.e., bilateral putamen/globus pallidus, as well as thalamus and caudate in the left hemisphere), and cerebellum. Bearing in mind that specific contrasts were chosen slightly differently, 
these results overlap substantially with the findings of Krämer and colleagues (2007). Activation of AIC in the TAP or similar fairness games has been associated with the processing of negative emotions such as anger and disgust (Krämer et al., 2007; Sanfey, Rilling, Aronson, Nystrom, \& Cohen, 2003; White et al., 2013). This follows a tradition of associating AIC with experience of mostly but not exclusively negative salient emotions (Craig, 2009; Damasio et al., 2000; Jabbi, Swart, \& Keysers, 2007). Furthermore, increased activation of subcortical structures such as the putamen/globus pallidus and the caudate as part of the striatum has been observed. This has previously been reported for punishment of unfair offers in a social interaction game investigating altruistic punishment (de Quervain et al., 2004). Krämer and colleagues (2007) argue that by punishing the provoking opponent participants might aim to modify the opponent's behaviour. In case the provoking opponent could be educated, his punishment towards the participant might decrease after consequent retaliation, which in turn would be rewarding for the participant. Besides AIC and subcortical activation, the inferior parietal lobe was significantly involved in retaliation. This could reflect putting oneself in the opponent's position. Especially the temporoparietal junction was repeatedly associated with theory of mind and social cognition (Corbetta et al., 2008). To understand the other's position and motivation is a prerequisite for the successful and dynamic adaption of behaviour within social interactions. In the context of the TAP as we implemented it in a healthy population, this is especially crucial when interacting with the provoking opponent. In order to adapt one's default reaction (choosing relatively low feedback as done so for the non-provoking opponent) and retaliate, one has to quickly identify the opponent, extract his putative motivation from his previous behaviour, and chose for the reasonable response. The relatively strong involvement of left inferior parietal lobe found in our data might reflect such a process amongst others. This explanation might be supported by the fact that inferior parietal lobe is the only activation hotspot within the provocation related neural network that was not at the same time involved in unsuccessful inhibition (see conjunction analysis). As a simple motor response task does not require social cognition of any kind, this finding might imply that the specific social component present in the TAP and absent in the GNGT is mirrored in inferior parietal activation.

Not just with respect to parietal involvement do our data show greater left- than right-hemispheric neural involvement during aggressive reactions to provocation. It might be interesting to consider this result in the light of theoretical frameworks based on inter-hemispheric balance and motivational direction. Approach motivation and anger on state as well as trait level are consistently reported to be accompanied by a shift in inter-hemispheric balance in favor of the left hemisphere (Carver \& HarmonJones, 2009; Eddie Harmon-Jones \& Sigelman, 2001; Eddie Harmon-Jones, 2004; Hortensius, Schutter, \& Harmon-Jones, 2012; van Honk \& Schutter, 2006). This is well mirrored in our results. On the other hand, in line with this perspective, successful 
inhibition, a cognitive process conceptually based on avoidance motivation, shows exclusively right-hemispheric neural correlates and, thus, the exact opposite pattern.

Anterior insula involvement. When investigating conjoint activity associated to disinhibition on the level of motor action and social interaction, bilateral insula cortex, and left-lateralized subcortical structures (i.e., thalamus and putamen/globus pallidus) revealed the strongest functional overlap.

The strong and constant involvement of AIC in motor impulsivity as well as reactive aggression is striking. Our data do not only show an activation overlap within this region, but also that the more a participant recruits right AIC during unsuccessful inhibition, the more it is recruited during aggressive behaviour. AIC has been target of numerous investigations across almost countless domains, including interoception, awareness of body movement, self-recognition, vocalization and music, emotional awareness, uncertainty and anticipation, visual and auditory awareness, time perception, attention, perceptual decision making, cognitive control, and performance monitoring (following the review of Craig, 2009). In the context of the present study the AIC has been repeatedly associated with successful inhibition (Sharp et al., 2010; Swick et al., 2011). Especially voluntary inhibition, so to speak the "free won't" as opposed to the free will (Brass \& Haggard, 2007), has been the function attributed to AIC activation. As mentioned previously, AIC involvement in the context of social interaction paradigms related to retaliation and the punishment of unfairness has mostly been interpreted as reflecting the processing of negative emotions (de Quervain et al., 2004; Krämer et al., 2007; Sanfey et al., 2003; White et al., 2013). These interpretations are highly reasonable, although seemingly conflicting with results that reveal AIC to be equally involved in unsuccessful inhibition (V Menon et al., 2001) and the processing of positive emotions (Hennenlotter et al., 2005; Jabbi et al., 2007).

Generally, taking a broader perspective on insula function might resolve these potential conflicts. All contexts for which significant anterior insula activation has been reported (Craig, 2009) require the ability to focus on the immediate presence and the involvement of rather unusual, potentially threatening, or change demanding stimuli. This involves an instantaneous need to monitor whether the current or planned behaviour is still adequate and if necessary to quickly adapt responses. Thus, saliency monitoring, response switching, attention, and control are key aspects in this process (Menon \& Uddin, 2010). In their review on anterior insula activation in perceptual paradigms, Sterzer and Kleinschmidt (2010) emphasize that anterior insula is recruited as soon as any perceptual input poses a challenge to the given modus operandi. Craig (2009) goes a step further by suggesting AIC to be the crucial node in a human awareness network whose main responsibility is the subjective regulation of psychological and physiological reactions to cognitively challenging situations. Following this reasoning, he proposes AIC even as potential candidate for the neural correlate of consciousness. Our data clearly supports the interpretation that AIC might be crucial for dynamic behavioural control across different modalities such as cognition and social interaction. 
Our findings provide evidence on neural level for the speculation Hoaken and colleagues (2003) derive from their behavioural data: More than inhibition as such, it might be rather general problems with the processing of social information that mediate the link between executive functioning and aggression. Both motor response inhibition as well as naturalistic aggressive reaction to provocation, constitute cognitive situations requiring high mental involvement and flexible adaption to rare respectively unusual and, thus, salient stimuli. Therefore, we interpret our findings as evidence for the involvement of anterior insula cortex not in specifically inhibitory, but general cognitive self-control across different modalities.

This does not imply that AIC not equally incorporates functions such as voluntary motor inhibition or the processing of negative emotions. It has to be taken into account that not only an aggressive reaction to provocation, but also dysfunctional motor inhibition, and thus failure in a specific task, could be accompanied by negative emotions. AIC has frequently been considered a crucial node of interoception in the cognitive system (Craig, 2009) and as such could merely reflect the processing of anger.

Reactive aggression does not only implicitly comprise a highly impulsive component. Aggression - despite its immediate, possibly functional consequences for the executor - is mostly considered unwanted in society and is highly stigmatized. For this reason, it often imposes negative long-term consequences on the aggressor. Therefore, it could be argued that in order to avoid these negative consequences, aggressive reactions to provocation should generally be inhibited. In the same line, a motor response should be inhibited in a response inhibition paradigm. In this respect, reactive aggression is closely related to motor impulsivity and a better grasping of this overlap can eventually lead to a better understanding of clinical syndromes involving aggression and other inhibitory deficits.

\section{Limitations}

The current findings and conclusions have to be considered bearing in mind the limitations of our study. We only examined 15 healthy, young males. Thus our findings cannot be generalized to bigger populations, to a gender-unspecific context, or any clinical population.

This study was designed to research the specific question in how far neural correlates of motor impulsivity and reactive aggression overlap. Nevertheless, it has to be emphasized that the interpretations of our results are of a rather exploratory nature and comprise several alternative explanations. This has to be kept in mind, when evaluating the common neural ground of impulsivity and aggression.

Furthermore, it has to be emphasized that despite their experimental advantages the chosen behavioural paradigms measure rather specific expressions of the underlying concepts. The GNGT is one among various paradigms employed to measure response inhibition. Eagle and colleagues (2008) differentiated several aspects of action 
inhibition, attributing action restraint to the GNGT as opposed to, for instance, action cancellation measured in stop signal paradigms. This should be kept in mind when interpreting our findings and only further research including different inhibition paradigms can allow for generalizations.

Despite providing us with a well-established tool to investigate provocative social interactions and retaliation in an imaging environment, it has to be considered that the TAP proves to be an unusually complex paradigm for an fMRI setting. For the presented analyses we focused merely on the decision phase of the task in which actual behaviour can be observed. Although our behavioural results show that the experimental manipulation was successful and a rather natural pattern of social interaction could be simulated, the limited variety of observed behaviour poses a challenge regarding the choice of maximally specific GLM contrasts. In order to ensure sufficient power of statistical analyses and maximal convergence towards real life social behaviour, we restricted our analyses to the contrast of aggressive behaviour in a provocative social interaction and not aggressive behaviour in a social interaction involving no provocation. Due to this circumstance, we cannot fully dissociate pure provocation and pure reactive aggression as for instance in the study by Krämer and colleagues (2007) in which punishment could be selected from only four levels instead of eighth in as in our study. It might be more likely that the entire scale is at least once administered for each opponent if there is less options to choose from. All conclusions we draw are based on perceiving retaliation and the provocative situation causing it as a unity. Therefore, the interpretations resulting from the presented findings definitely lack in specificity. It might, though, be questionable, whether neural components exclusively involved in pure inhibition or pure reactive aggression exist and whether identifying them will ultimately enable us to better understand such complex real life concepts as self-control and retaliation. For future studies, it would be rather interesting to look at participants administering relatively greater punishments than their peers and at the same time display a weaker ability to inhibit motor responses. While enabling a more precise description of the specific overlap between the two domains, this would require a significantly larger sample than our study features.

\section{CONCLUSION AND IMPLICATIONS}

In this study we investigated the neural correlates involved in successful and unsuccessful response inhibition as well as retaliation towards a provoking social counterpart. Furthermore, we focused on the overlap of neural components involved in disinhibition in the context of motor action and provocation. Our findings provide evidence of anterior insula involvement in general self-control across different domains including motor action and social interaction. This might enable a broader perspective on insula function in terms of the awareness model proposed by Craig (2009). 
Anderson, C. A., Lindsay, J. J., \& Bushman, B. J. (1999). Research in the Psychological Laboratory: Truth or Triviality? Current Directions in Psychological Science, 8(1), 3-9.

Aron, A. R., Robbins, T. W., \& Poldrack, R. A. (2004). Inhibition and the right inferior frontal cortex. Trends in Cognitive Sciences, 8(4), 170-7.

Asahi, S., Okamoto, Y., Okada, G., Yamawaki, S., \& Yokota, N. (2004). Negative correlation between right prefrontal activity during response inhibition and impulsiveness: a fMRI study. European Archives of Psychiatry and Clinical Neuroscience, 254(4), 245-51.

Bernstein, S., Richardson, D., \& Hammock, G. (1987). Convergent and discriminant validity of the Taylor and Buss measures of physical aggression. Aggressive Behavior, 13(1), 15-24.

Brass, M., \& Haggard, P. (2007). To do or not to do: the neural signature of self-control. The Journal of Neuroscience, 27(34), 9141-5.

Carver, C. S., \& Harmon-Jones, E. (2009). Anger is an approach-related affect: evidence and implications. Psychological Bulletin, 135(2), 183-204.

Chambers, C. D., Garavan, H., \& Bellgrove, M. a. (2009). Insights into the neural basis of response inhibition from cognitive and clinical neuroscience. Neuroscience and Biobehavioral Reviews, 33(5), 631-46.

Chikazoe, J., Konishi, S., Asari, T., Jimura, K., \& Miyashita, Y. (2007). Activation of right inferior frontal gyrus during response inhibition across response modalities. Journal of Cognitive Neuroscience, 19(1), 69-80.

Corbetta, M., Patel, G., \& Shulman, G. L. (2008). The reorienting system of the human brain: from environment to theory of mind. Neuron, 58(3), 306-24.

Craig, A. D. B. (2009). How do you feel - now? The anterior insula and human awareness. Nature Reviews Neuroscience, 10(1), 59-70.

Damasio, A. R., Grabowski, T. J., Bechara, A., Damasio, H., Ponto, L. L., Parvizi, J., \& Hichwa, R. D. (2000). Subcortical and cortical brain activity during the feeling of self-generated emotions. Nature Neuroscience, 3(10), 1049-56.

De Quervain, D. J.-F., Fischbacher, U., Treyer, V., Schellhammer, M., Schnyder, U., Buck, A., \& Fehr, E. (2004). The neural basis of altruistic punishment. Science, 305(5688), 1254-8.

Deichmann, R., Gottfried, J. A., Hutton, C., \& Turner, R. (2003). Optimized EPI for fMRI studies of the orbitofrontal cortex. Neurolmage, 19(2), 430-41.

Eagle, D. M., Bari, A., \& Robbins, T. W. (2008). The neuropsychopharmacology of action inhibition: crossspecies translation of the stop-signal and go/no-go tasks. Psychopharmacology, 199(3), 439-56.

Eichele, T., Debener, S., Calhoun, V. D., Specht, K., Engel, A. K., Hugdahl, K., ... Ullsperger, M. (2008). Prediction of human errors by maladaptive changes in event-related brain networks. National Academy of Sciences of the United States of America, 105(16), 6173-8.

Forman, S. D., Cohen, J. D., Fitzgerald, M., Eddy, W. F., Mintun, M. A., \& Noll, D. C. (1995). Improved assessment of significant activation in functional magnetic resonance imaging (fMRI): use of a cluster-size threshold. Magnetic Resonance in Medicine, 33(5), 636-47.

Giancola, P. R., \& Parrott, D. J. (2008). Further evidence for the validity of the Taylor Aggression Paradigm. Aggressive Behavior, 34(2), 214-29.

Giancola, P. R., \& Zeichner, A. (1995). Construct validity of a competitive reaction-time aggression paradigm. Aggressive Behavior, 21(3), 199-204.

Harmon-Jones, E. (2004). Contributions from research on anger and cognitive dissonance to understanding the motivational functions of asymmetrical frontal brain activity. Biological Psychology, 67(1-2), 51-76.

Harmon-Jones, E., \& Sigelman, J. (2001). State anger and prefrontal brain activity: evidence that insultrelated relative left-prefrontal activation is associated with experienced anger and aggression. Journal of Personality and Social Psychology, 80(5), 797-803.

Hennenlotter, A., Schroeder, U., Erhard, P., Castrop, F., Haslinger, B., Stoecker, D., ... Ceballos-Baumann, A. O. (2005). A common neural basis for receptive and expressive communication of pleasant facial affect. Neurolmage, 26(2), 581-91.

Hoaken, P. N. S., Shaughnessy, V. K., \& Pihl, R. O. (2003). Executive cognitive functioning and aggression: Is it an issue of impulsivity? Aggressive Behavior, 29(1), 15-30. 


\section{CHAPTER 4}

Horn, N. R., Dolan, M., Elliott, R., Deakin, J. F. W., \& Woodruff, P. W. R. (2003). Response inhibition and impulsivity: an fMRI study. Neuropsychologia, 41(14), 1959-66.

Hortensius, R., Schutter, D. J. L. G., \& Harmon-Jones, E. (2012). When anger leads to aggression: induction of relative left frontal cortical activity with transcranial direct current stimulation increases the angeraggression relationship. Social Cognitive and Affective Neuroscience, 7(3), 342-7.

Jabbi, M., Swart, M., \& Keysers, C. (2007). Empathy for positive and negative emotions in the gustatory cortex. Neurolmage, 34(4), 1744-53.

Klein, T. a, Endrass, T., Kathmann, N., Neumann, J., von Cramon, D. Y., \& Ullsperger, M. (2007). Neural correlates of error awareness. Neurolmage, 34(4), 1774-81.

Krämer, U. M., Jansma, H., Tempelmann, C., \& Münte, T. F. (2007). Tit-for-tat: the neural basis of reactive aggression. Neurolmage, 38(1), 203-11.

Krämer, U. M., Kopyciok, R. P. J., Richter, S., Rodriguez-Fornells, A., \& Münte, T. F. (2011). The role of executive functions in the control of aggressive behavior. Frontiers in Psychology, 2(7), 152.

Logan, G. D., Schachar, R. J., \& Tannock, R. (1997). Impulsivity and Inhibitory Control. Psychological Science, 8(1), 60-64.

Lotze, M., Veit, R., Anders, S., \& Birbaumer, N. (2007). Evidence for a different role of the ventral and dorsal medial prefrontal cortex for social reactive aggression: An interactive fMRI study. Neurolmage, 34(1), 470-8.

Menon, V., Adleman, N. E., White, C. D., Glover, G. H., \& Reiss, a L. (2001). Error-related brain activation during a Go/NoGo response inhibition task. Human Brain Mapping, 12(3), 131-43.

Menon, V., \& Uddin, L. Q. (2010). Saliency, switching, attention and control: a network model of insula function. Brain Structure \& Function, 214(5-6), 655-67.

Pawliczek, C. M., Derntl, B., Kellermann, T., Kohn, N., Gur, R. C., \& Habel, U. (2013). Inhibitory control and trait aggression: Neural and behavioral insights using the emotional stop signal task. Neurolmage, 79, 264-274.

Sanfey, A. G., Rilling, J. K., Aronson, J. A, Nystrom, L. E., \& Cohen, J. D. (2003). The neural basis of economic decision-making in the Ultimatum Game. Science, 300(5626), 1755-8.

Sharp, D. J., Bonnelle, V., De Boissezon, X., Beckmann, C. F., James, S. G., Patel, M. C., \& Mehta, M. a. (2010). Distinct frontal systems for response inhibition, attentional capture, and error processing. National Academy of Sciences of the United States of America, 107(13), 6106-11.

Simmonds, D. J., Pekar, J. J., \& Mostofsky, S. H. (2008). Meta-analysis of Go/No-go tasks demonstrating that fMRI activation associated with response inhibition is task-dependent. Neuropsychologia, 46(1), 22432.

Sterzer, P., \& Kleinschmidt, A. (2010). Anterior insula activations in perceptual paradigms: often observed but barely understood. Brain Structure \& Function, 214(5-6), 611-22.

Swick, D., Ashley, V., \& Turken, U. (2011). Are the neural correlates of stopping and not going identical? Quantitative meta-analysis of two response inhibition tasks. Neurolmage, 56(3), 1655-65.

Talairach, J., \& Tournoux, P. (1988). Co-planar stereotaxic atlas of the human brain: an approach to medical cerebral imaging.

Taylor, S. P. (1967). Aggressive behavior and physiological arousal as a function of provocation and the tendency to inhibit aggression. Journal of Personality, 35(2), 297-310.

Van Honk, J., \& Schutter, D. J. L. G. (2006). From affective valence to motivational direction: the frontal asymmetry of emotion revised. Psychological Science, 17(11), 963-5.

Veit, R., Lotze, M., Sewing, S., Missenhardt, H., Gaber, T., \& Birbaumer, N. (2010). Aberrant social and cerebral responding in a competitive reaction time paradigm in criminal psychopaths. Neurolmage, 49(4), 3365-72.

Weissman, D. H., Roberts, K. C., Visscher, K. M., \& Woldorff, M. G. (2006). The neural bases of momentary lapses in attention. Nature Neuroscience, 9(7), 971-8.

White, S. F., Brislin, S. J., Meffert, H., Sinclair, S., \& Blair, R. J. R. (2013). Callous-unemotional traits modulate the neural response associated with punishing another individual during social exchange: a preliminary investigation. Journal of Personality Disorders, 27(1), 99-112. 




\section{CHAPTER 5 No effects of bilateral tDCS over inferior frontal gyrus on response inhibition and aggression}

"Science, my boy, is made up of mistakes, but they are mistakes which are useful to make, because they lead little by little to the truth."

- Jules Verne, Journey to the Center of the Earth, 1864 
Based on: Dambacher*, F., Schuhmann*, T., Lobbestael, J., Arntz, A., Brugman, S., \& Sack, A. T. (2015). No effects of bilateral tDCS over inferior frontal gyrus on response inhibition and aggression. submitted

* both authors contributed equally

We thank Anke Lemmens for her helpful support. 


\section{ABSTRACT}

Brain imaging studies have shown that in both, response inhibition and aggression, the prefrontal cortex is consistently activated. Response inhibition describes the capacity to adequately inhibit or restraint automatic or pre-planned responses. Deficits in this capacity have been linked to aggressive behaviour. Pro- and reactive aggression are sub-types of aggression, describing the display of aggressive behaviour either instrumentally or in response to provocation. Response inhibition is mostly associated with predominantly right prefrontal activity the neural components underlying aggression seem to be left-lateralized. These differences in hemispheric dominance are conceptualized in cortical asymmetry theories on motivational direction, which assign avoidance motivation (relevant to inhibit responses) to the right and approach motivation (relevant for aggressive actions) to the left prefrontal cortex. In line with this notion, previous studies employing Non-Invasive Brain Stimulation techniques demonstrated that unilateral enhancement of the right prefrontal cortex can increase inhibitory capacity and reduce aggression. So far no brain stimulation study aimed to directly address the inherent inverse relationship between both concepts by assessing response inhibition and aggression within one experiment and by applying two opposing bilateral brain stimulation protocols in order to respectively induce left or right fronto-cortical dominance. In the current study, sixty-nine healthy participants underwent bilateral transcranial Direct Current Stimulation (tDCS) with anode over left and cathode over right inferior frontal cortex or vice versa (inducing either left- or right-hemispheric dominance). Sham stimulation was administered to the control group. During brain stimulation, response inhibition as well as pro- and reactive aggression were assessed with a go/no-go task (GNGT) and the Taylor Aggression Paradigm (TAP). On a behavioural level, in the group receiving sham stimulation, we revealed an inverse relationship between response inhibition and aggressive behaviour; the worse the ability to restrain a response in the GNGT, the more aggression was displayed during the TAP. No effects of bilateral prefrontal tDCS on either response inhibition or aggression were observed. This is at odds with previous unilateral brain stimulation studies, and therefore fails to provide evidence in support of the prefrontal cortical asymmetry model in the domain of response inhibition and aggression. The absence of tDCS effects might furthermore indicate that the methodological approach of shifting cortical asymmetry by means of bilateral tDCS protocols has failed, and/or that targeting inferior frontal cortex instead of the dorsolateral prefrontal cortex is not advisable. These and other possible explanations for our results and indications for brain stimulation research are shortly discussed. 


\section{CHAPTER 5}

Response inhibition is defined as the cognitive ability to withhold automatic or preplanned reactions (Logan et al., 1997) and comprises various sub-components,such as ation cancellation and action restraint. While action cancellation refers to the withdrawal of an already initiated response, action restraint refers to the withdrawal of an action prior to its initiation (Eagle et al., 2008). The latter is mirrored in a variety of real life behaviours especially in the social domain, where a reaction might be imminent, but has to be taken back at a last possible notice before it is executed. Action restraint is classically measured by go/no-go paradigms in which participants have to respond to a frequent go stimulus, while they have to restrain their response to an infrequent nogo stimulus.

Aggressive acts are one concrete form of social interactions, in which response inhibition is of importance. Aggression is understood as behaviour that aims to intentionally harm another being verbally, physically, or psychologically (Anderson \& Bushman, 2002). As the term aggression comprises rather complex aspects of behaviour, it is usually categorized into sub-types; while proactive aggression refers to using aggression in an instrumental, goal-oriented way, reactive aggression refers to aggressive actions in response to preceding provocation (Poulin \& Boivin, 2000; Raine et al., 2006). A tool to measure aggression, going beyond mere self-report and towards a controlled behavioural assessment, is the Taylor Aggression Paradigm (TAP; Taylor, 1967). The TAP is set up as a reaction time game in which two or more opponents interact and are enabled to administer aversive feedback of variable intensity to each other.

Response inhibition and aggression are related on various levels. On the behavioural level, the display of aggression has empirically been associated with impaired executive functioning including response inhibition (Hoaken, Shaughnessy, \& Pihl, 2003). On the neural level, neuroscience has investigated how response inhibition relates to self-reported impulsivity and aggression. For instance, Horn and colleagues (2003) showed that impulsive compared to non-impulsive individuals recruited more activity in the right orbitofrontal cortex to maintain inhibitory capacities in a go/no-go task (GNGT). Furthermore, GNGT-inhibition related activity in the right dorsolateral prefrontal cortex was shown to negatively correlate with impulsiveness on the Barratt Impulsiveness Scale (Asahi, Okamoto, Okada, Yamawaki, \& Yokota, 2004). Pawliczek and colleagues (2013) demonstrated that highly aggressive individuals show inhibition deficits in an emotional stop signal task on behavioural level and on neural level lower inhibition related brain activity in pre-supplementary motor area and primary motor cortex. More recently, we could demonstrate a substantial overlap of neural networks involved in failed response inhibition and behavioural aggression within anterior insula and various sub-cortical brain regions (Dambacher, Sack, Lobbestael, Arntz, Brugman, \& Schuhmann, 2014b).

Despite the described relationship between response inhibition and aggression, neuroscientific research has mostly examined the concepts in isolation. In brain imag- 
ing literature, response inhibition is mainly associated with activity in the right prefrontal cortex (Aron, Robbins, \& Poldrack, 2004; Dambacher, Sack, Lobbestael, Arntz, Brugman, \& Schuhmann, 2014a), while anger and aggression are mainly associated with activity in the left prefrontal cortex (Carver \& Harmon-Jones, 2009; Dambacher, Sack, Lobbestael, Arntz, Brugman, \& Schuhmann, 2014b; van Honk \& Schutter, 2006).

In a similar vein, studies employing Non-Invasive Brain Stimulation (NIBS) such as transcranial Direct Current Stimulation (tDCS) and Transcranial Magnetic Stimulation (TMS) suggest that response inhibition can be altered by enhancing or inhibiting activity in the right prefrontal cortex (thus, shifting the cortical balance towards or away from the right prefrontal cortex). For instance, Jacobson and colleagues (2011) showed that enhancing activity in right inferior frontal gyrus by means of unilateral anodal tDCS improved inhibition in a stop signal task. In contrast, the disruption of right inferior frontal cortex by means of TMS (Chambers et al., 2006, 2007; Dambacher, Sack, Lobbestael, Arntz, Brugmann, \& Schuhmann, 2014; Verbruggen, Aron, Stevens, \& Chambers, 2010) impaired successful inhibition in various response inhibition paradigms. Similar results were found when inhibiting right dorsolateral prefrontal cortex by means of cathodal tDCS (Beeli, Casutt, Baumgartner, \& Jäncke, 2008). When studying the concept of aggression, NIBS findings suggest that aggression and its cognitive predecessors can be increased by shifting the front-cortical balance towards the left hemisphere and decreased when shifting it to the right: D'Alfonso and colleagues (2000) demonstrated that by disrupting the right prefrontal cortex by means of repetitive TMS they could induce an attentional bias towards angry faces, while the disruption of left prefrontal cortex had an opposite effect. When enhancing the left prefrontal cortex with tDCS, aggressive behaviour in provocative situations was increased (Hortensius, Schutter, \& Harmon-Jones, 2012). Opposite effects (decrease of proactive aggression in males) were observed, when unilaterally enhancing the right dorsolateral prefrontal cortex (Dambacher et al., 2015).

In summary, studies employing NIBS showed that shifting the fronto-cortical dominance towards the right hemisphere (by enhancing the right and/or disrupting the left prefrontal cortex) increases inhibitory capacity and decreases aggression, while shifting it to the left (by enhancing the left and/or disrupting the right prefrontal cortex) had the exact opposite effect. This evidence is in line with the theoretical framework on fronto-cortical asymmetry and motivational states proposed by Harmon-Jones and colleagues (Harmon-Jones \& Allen, 1998; Harmon-Jones \& Sigelman, 2001; HarmonJones, 2004). It states that avoidance motivation is associated with right-, while approach motivation is with left-hemispheric fronto-cortical brain activity. Although the fact that action restraint and aggression seem inversely related on behavioural and neural level fits with this framework, no brain stimulation study until today aimed to directly address this relationship by assessing both concepts within one experiment and by applying two opposing bilateral brain stimulation protocols in order to respectively induce left and right fronto-cortical dominance. 


\section{CHAPTER 5}

In the current study, we applied bilateral tDCS (inducing either right or left frontocortical dominance) or sham stimulation, while participants were performing a standard GNGT and the TAP. Based on previous neuroimaging work (Dambacher, Sack, Lobbestael, Arntz, Brugman, and Schuhmann, 2014b), stimulation was targeted at the inferior frontal cortex. It was assumed that response inhibition and aggression were inversely related on behavioural and neural level. We hypothesized that the induction of right-hemispheric fronto-cortical dominance by means of anode over right / cathode over left bilateral tDCS would enhance inhibitory capacity and simultaneously reduce aggressive behaviour. Moreover, we expected that the induction of left-hemispheric fronto-cortical dominance by means of anode over left / cathode over right bilateral tDCS would impair inhibitory capacity and simultaneously increase aggressive behaviour.

\section{METHODS AND MATERIALS}

Participants. Sixty-nine healthy volunteers participated in this study. Data of one participant was incomplete due to technical problems and had to be excluded from the analysis. Another four participants were not included in the analysis, as they doubted the interaction with a real human opponent during the TAP (see below). Therefore, sixty-four participants (mean age in years $=21.89$; SD $=3.26$ ) were included in the analysis. Participants were randomly assigned to one of three experimental groups (between-subject design): One group received anodal stimulation over right and simultaneously cathodal stimulation over left inferior frontal cortex (induction of righthemispheric dominance; male $n=11$ female $n=11$ ). One group received anodal stimulation over left and simultaneously cathodal stimulation over right inferior frontal cortex (induction of left-hemispheric dominance; male $n=14$ female $n=8$ ). A third group received sham stimulation (male $n=14$ female $n=6$ ).

Participants had no history of neurological or psychiatric disorders, were suited to undergo Non-Invasive Brain Stimulation, and gave their written informed consent before taking part. They were paid for participation.

Paradigms and tools. GNGT. In order to measure response inhibition or action restraint, a standard go/no-go motor response task was employed (Dambacher, Sack, Lobbestael, Arntz, Brugmann, \& Schuhmann, 2014c). Participants were instructed to respond as fast and accurately as possible to a frequent go stimulus via button press, while they had to restrain their response to a rare no-go stimulus. Go as well as no-go stimuli were presented for $100 \mathrm{msec}$. Inter trial intervals were randomly varied (650, $750,850,950$, or $1050 \mathrm{msec}$ ) eliminating expectancy effects. The letters C and M were used as stimuli, as they lack any linguistic association with the concept of "stopping" in the languages spoken by the participants. Stimuli and fixation crosses were presented in white (RGB 255/255/255; Arial pt 24) on a grey background (RGB 125/125/125). For 
both the baseline and the actual experimental measurement, participants had to complete 5 blocks of 64 trials including 25\% inhibition trials. Go and no-go trials were pseudo-randomized (one of four trials was an inhibition trial). This design led to a total of 320 trials (80 inhibition trials). After each block participants received feedback on their mean reaction times for go trials, their number of omission errors in go trials, and their percentage of commission errors in inhibition trials. Stimuli were presented using Presentation software (Neurobehavioural Systems, Inc., Albany, USA).

TAP. In order to measure actual aggressive behaviour, the Taylor Aggression Paradigm (TAP; Taylor, 1967) implemented in custom-made software was employed. It was framed as a competitive reaction time game in which participants had to respond to a target stimulus as fast as possible by button press with the right index finger. Two participants were simultaneously invited to the laboratory. Each participant was told to play against another participant of the same gender sitting in the next room. The amount of win and lose trials were preprogrammed in the same order for every participant. Participants were made to believe that the winner of a trial could administer an aversive noise to the opponent and that this noise could influence the performance of the opponent on the next trial. In the beginning of each trial, the participant was asked to choose the duration and volume of this noise blast by moving a slider on a scale from 0 to 10 (duration: 0 to 5 seconds; volume: 0 to $100 \mathrm{~dB}$ ). At the end of each trial, participants were informed about whether they had won or lost the trial. At the same time they could see which feedback the opponent had chosen for the trial. In the case that the participant had lost, she / he was presented with this feedback through headphones. By summing and averaging the behaviour (given intensity \& duration) across all trials, a total aggression score was calculated. The behaviour (given intensity \& duration) across the first seven unprovoked trials (in which the opponent never administered a noise) was summed up and averaged to receive a proactive aggression score. By summing and averaging the behaviour (given intensity \& duration) from the eighth trial onwards (provoked trials), a reactive aggression score was calculated. The TAP demonstrated high construct, internal, discriminant as well as external validity (Anderson, Lindsay, \& Bushman, 1999; Bernstein, Richardson, \& Hammock, 1987; Giancola \& Parrott, 2008; Giancola \& Zeichner, 1995).

Questionnaires. The Reactive-Proactive-Aggression Questionnaire (RPQ) was used to measure self-reported trait aggression (Raine et al., 2006). Participants rated their opponents regarding sympathy, competence, friendliness, and reaction time speed on a 7-point likert scale at the beginning and the end of the experiment. In order to check, whether the actual behaviour (administration of feedback noise) and not merely the perception of the feedback received by the opponent was modulated by tDCS, participants rated on a 7-point likert scale how annoying 4 exemplary feedbacks would have been for them, if they would have received them during the game ('volume 0 / duration 0 ', 'volume 4 / duration 2', 'volume 10 / duration 10', 'volume 2 / duration 6'). During this rating the brain stimulation was still active. 
Non-Invasive Brain Stimulation. Participants were divided into three groups and randomly assigned to one of three tDCS conditions: induction of right-hemispheric fronto-cortical dominance, induction of left-hemispheric fronto-cortical dominance, or sham stimulation.

To induce right-hemispheric fronto-cortical dominance, the anode was positioned over right inferior frontal cortex (F8), while the cathode was positioned over left inferior frontal cortex (F7). To induce left-hemispheric fronto-cortical dominance, the anode was positioned over left inferior frontal cortex (F7), while the cathode was positioned over right inferior frontal cortex (F8). These stimulation sites were chosen in accordance with existing imaging work allocating the main overlap of neural networks involved in response inhibition and aggression in inferior frontal regions (Dambacher, Sack, Lobbestael, Arntz, Brugman, \& Schuhmann, 2014a, 2014b). A DC-stimulator plus and $5 \times 7 \mathrm{~cm}$ standard electrodes (neuroConn, Ilmenau, Germany) were employed. Electrodes were fixated using conductive EEG gel (Ten20 conductive Neurodiagnostic electrode Paste, WEAVER and company, Aurora CO, USA). We applied 1.5mA direct current for 21.75 minutes, including ramping up and down phases of 20 s each.

When administering sham tDCS the electrodes were equally positioned, but the stimulation was switched of immediately after the ramping phase. Therefore, participants also had a light skin sensation in the sham condition and could not differentiate whether they had been assigned to the real or the sham tDCS condition. When rating how certain they were about which type of stimulation they received (from 1 "100\% sham" to 7 "100\% real"; with 4 "I don't know"), no differences between the real and sham stimulation groups were identified (right-hemispheric fronto-cortical dominance: $M E A N=4.73$; left-hemispheric fronto-cortical dominance: $M E A N=4.67$; sham stimulation: MEAN=4.75; ANOVA: $F(2,61)=.017 \mathrm{p}=.984)$.

Experimental procedure. Participants were told that they took part in a study investigating the effects of human feedback compared to computerized feedback in reaction time performance. In each experimental session two participants of the same gender that did not know each other took part simultaneously. Participants were seated in two different laboratory rooms next to each other. Following the montage of the tDCS setup, participants received instructions and completed a baseline measurement of the GNGT prior to the initiation of the stimulation. During brain stimulation (the experimental manipulation) participants performed the GNGT and the TAP in counterbalanced order. Subsequently, participants had to fill in the questionnaires. Immediately after completion of the experiment, participants were administered an exit interview ensuring that they were fully deceived by the experimental setup. After all participants were assessed, participants were provided with a written debriefing. The study was approved by the local Ethical Committee of the Faculty of Psychology and Neuroscience at Maastricht University.

Statistical analysis. For response inhibition, the effects of brain stimulation were examined with a $3 \times 2$ multivariate analysis of variance (MANOVA; conditions $x$ gender; 
with mean reaction time on go trials, misses, and false alarms as dependent variables; all corrected for baseline via differential scores). For aggression, the effects of brain stimulation were examined with a $3 \times 2$ multivariate analysis of variance (MANOVA; conditions $x$ gender; with total aggression, proactive aggression, and reactive aggression as dependent variables); trait aggression (RPQ) was included as a covariate. Specific differences were examined with paired-sample t-tests. Relationships were investigated via Pearson product-moment correlation coefficients. Post hoc analyses were conducted with G-power software (Faul et al., 2007) to ensure sufficient statistical power.

\section{RESULTS}

A relation between response inhibition and aggression could be observed. Within the sham condition, inhibitory capacity in the GNGT correlated with the behaviour across all types of aggression in the TAP; the more false alarms participants committed, the more aggression they displayed (TOTAL AGGRESSION $r=.550 p=.012$ / PROACTIVE AGGRESSION $r=.452 p=.046$ / REACTIVE AGGRESSION $r=.474 p=.035$ ). No such relation could be observed within the groups receiving real stimulation. It has to be noted that participants in the group that received sham stimulation became faster and committed more commission errors (false alarms) in the experimental measurement compared to the baseline measurement (reaction time: MEANpre=292.21 MEANpost=271.83 $\mathrm{t}(19)=5.686 \mathrm{p}<.001$; false alarms: MEANpre=22.20 MEANpost $=30.00 \mathrm{t}(19)=-3.765$ $\mathrm{p}=.001$ ). Therefore, in all three groups further analyses for the GNGT were computed on differential scores.

Effects of brain stimulation. Means and standard deviations are depicted in table 1. For response inhibition, a 3×2 MANOVA (conditions $x$ gender; with mean reaction time on go trials, misses, and false alarms as dependent variables; all corrected for baseline) revealed no significant main effects (MEAN REACTION TIME condition: $F(2,61)=2.293 p=.100$; gender: $F(1,62)=2.100 p=.153$ / MISSES condition: $F(2,61)=1.118$ $p=.334$; gender: $F(1,62)=1.949 p=.168$ / FALSE ALARMS condition: $F(2,61)=2.193$ $p=.121$; gender: $F(1,62)=.341 p=.562)$. Furthermore, no interaction effects could be observed (MEAN REACTION TIME condition*gender: $\mathrm{F}=.064 \mathrm{p}=.938$ / MISSES condition*gender: $\mathrm{F}=2.550 \mathrm{p}=.087$ / FALSE ALARMS condition*gender: $\mathrm{F}=.032 \mathrm{p}=.969)$ ). Posthoc power analyses revealed an achieved power of .95 for both main effects and the interaction (assuming alpha $=.05,1$-beta $=.95$, based on Pillai's $V$ per effect; calculated with G-Power). 
Tab. 1. Means and standard deviations. Reaction times, misses, and false alarms are represented as differential values (minus baseline performance). Agg: aggression.

\begin{tabular}{|c|c|c|c|c|c|c|c|c|c|c|c|c|}
\hline & \multicolumn{4}{|c|}{$\begin{array}{l}\text { induction of right-hemispheric } \\
\text { dominance }\end{array}$} & \multicolumn{4}{|c|}{$\begin{array}{l}\text { induction of left-hemispheric } \\
\text { dominance }\end{array}$} & \multicolumn{4}{|c|}{ sham stimulation } \\
\hline & \multirow{2}{*}{\multicolumn{2}{|c|}{$\begin{array}{l}\text { male } \\
\mathrm{n}=11\end{array}$}} & \multirow{2}{*}{\multicolumn{2}{|c|}{$\begin{array}{l}\text { female } \\
\mathrm{n}=11\end{array}$}} & \multirow{2}{*}{\multicolumn{2}{|c|}{$\begin{array}{l}\text { male } \\
\mathrm{n}=14\end{array}$}} & \multirow{2}{*}{\multicolumn{2}{|c|}{$\begin{array}{l}\text { female } \\
\mathrm{n}=8\end{array}$}} & \multirow{2}{*}{\multicolumn{2}{|c|}{$\begin{array}{l}\text { male } \\
\mathrm{n}=14\end{array}$}} & \multirow{2}{*}{\multicolumn{2}{|c|}{$\begin{array}{l}\text { female } \\
\mathrm{n}=6\end{array}$}} \\
\hline & & & & & & & & & & & & \\
\hline & M & SD & $M$ & SD & $M$ & SD & M & SD & $\mathrm{M}$ & SD & $M$ & SD \\
\hline reaction time & -22.47 & 12.50 & -30.84 & 21.88 & -9.49 & 23.61 & -18.20 & 19.70 & -19.00 & 16.57 & -23.61 & 15.64 \\
\hline misses & -.27 & 12.50 & -10.55 & 7.78 & -4.14 & 8.49 & -1.38 & 10.36 & 0.50 & 9.20 & -2.33 & 6.34 \\
\hline false alarms & 4.55 & 5.26 & 6.36 & 8.04 & 2.29 & 6.53 & 2.88 & 8.98 & 7.43 & 10.65 & 8.67 & 5.50 \\
\hline total agg & 5.10 & 1.31 & 3.85 & 1.05 & 4.26 & 1.73 & 2.98 & 1.52 & 4.45 & 1.18 & 4.73 & .90 \\
\hline proactive agg & 4.19 & 1.76 & 2.39 & 1.32 & 3.52 & 1.62 & 1.82 & .95 & 3.37 & 1.66 & 3.35 & .75 \\
\hline reactive agg & 5.33 & 1.26 & 4.22 & 1.05 & 4.45 & 1.89 & 3.27 & 1.86 & 4.73 & 1.33 & 5.07 & 1.06 \\
\hline
\end{tabular}

For aggression, a 3×2 MANOVA (conditions $x$ gender; with total aggression, proactive aggression and reactive aggression as dependent variable and trait aggression as covariate) revealed a significant gender difference in proactive aggression with males displaying more proactive aggression than females (PROACTIVE AGGRESSION gender: $F(1,62)=7.142 p=.010)$. No further significant main effects were revealed (TOTAL AGGRESSION condition: $F(2,61)=1.906 p=.159$; gender: $F(1,62)=3.459 p=.068$ / PROACTIVE AGGRESSION condition: $F(2,61)=1.060 p=.354$ / REACTIVE AGGRESSION condition: $F(2,61)=1.759 p=.182$; gender: $F(1,62)=2.07 p=.155)$. Furthermore, no interaction effects could be observed (TOTAL AGGRESSION condition*gender: $F=.960 p=.389$ / PROACTIVE AGGRESSION condition*gender: $F=1.575 p=.216$ / REACTIVE AGGRESSION condition*gender: $\mathrm{F}=.645 \mathrm{p}=.529)$. Post-hoc power analyses revealed an achieved power of .95 for both main effects and the interaction (assuming alpha=.05, 1-beta=.95, based on Pillai's V per effect; calculated with G-Power).

Control variables. Participants considered their opponent more competent, but less friendly after (as compared to before) they had played the TAP (How competent is your opponent pre/post: MEANpre=4.98 MEANpost=5.50 / $\mathrm{t}(63)=-3.014 \mathrm{p}=.004$; How friendly is your opponent pre/post: MEANpre=5.67 MEANpost=4.83 / $\mathrm{t}(63)=5.294 \mathrm{p}=.000$; all items rated on a 7point Likert-Scale). No significant differences in how much participants liked their opponent and how fast they thought the opponent is were observed (How much do you like your opponent pre/post: $\mathrm{t}(63)=.904 \mathrm{p}=.369$; How fast in his reactions will (was) your opponent be pre/post: $\mathrm{t}(63)=1.263 \mathrm{p}=.211$ ). The higher in volume and duration the feedback was, the more annoying it was perceived; this observation did not differ with respect to the brain stimulation conditions ("How annoying would this feedback have been for you?" rated between 1 "not at all" and 7 "very much"; right-hemispheric fronto-cortical dominance: feedback volume 0 \& duration 0 MEAN=1.50 / feedback volume 4 \& duration 1 MEAN=2.59 / feedback volume 10 \& 
duration 5 MEAN=6.59 / feedback volume 2 \& duration 3 MEAN=2.64; left-hemispheric fronto-cortical dominance: feedback volume 0 \& duration 0 MEAN=1.64 / feedback volume 4 \& duration $1 \mathrm{MEAN}=2.68$ / feedback volume 10 \& duration $5 \mathrm{MEAN}=6.36$ / feedback volume 2 \& duration $3 \mathrm{MEAN}=2.68$; sham stimulation: feedback volume 0 \& duration 0 MEAN=1.30 / feedback volume 4 \& duration 1 MEAN=2.70 / feedback volume 10 \& duration 5 MEAN=6.30 / feedback volume 2 \& duration 3 MEAN=2.65).

\section{DISCUSSION}

This study investigated to what degree shifting fronto-cortical balance by means of bilateral tDCS affects response inhibition and aggression. It was assumed that response inhibition and aggression are inversely related on behavioural and neural level. We expected that the induction of right-hemispheric fronto-cortical dominance (placing the anode over the right and the cathode over the left inferior frontal cortex in a bilateral tDCS setup) would lead to an enhanced ability to inhibit motor responses and at the same time reduce aggressive behaviour. Furthermore, the induction of lefthemispheric fronto-cortical dominance (placing the anode over the left and the cathode over the right inferior frontal cortex) was expected to reduce the ability to inhibit motor responses and at the same time increase aggressive behaviour. Our results failed to reveal any behavioural effects of either tDCS condition on response inhibition and/or aggression, and therefore do not provide any empirical support for these specific hypotheses.

Interestingly, independent of brain stimulation, we could observe the expected inverse relationship of the ability to inhibit pre-planned motor responses and all types of behavioural aggression (proactive, reactive, and total aggression). The more false alarms (commission errors) were committed by a given participant in the GNGT, the more aggression was displayed in the TAP. In other words: the worse people were in restraining responses when asked to do so, the more aggressively they behaved towards their opponent after provocation. This is in line with previous work associating response inhibition deficits with impulsivity (Asahi et al., 2004; Horn et al., 2003; Pawliczek et al., 2013). The current study, however, is the first to demonstrate a similar relationship with respect to impulsive aggression employing an actual behavioural instead of a merely self-reported measure.

The interaction during the reaction time game caused participants to evaluate their opponents as less friendly but more competent compared to the beginning of the experiment. This emphasizes that the situation might have been perceived as rather competitive and provocative. Furthermore, the louder and longer the feedback noises, the more annoying participants rated them. Both results indicate that the implementation of provocation in the TAP was successful. 


\section{CHAPTER 5}

We observed a tendency towards a gender effect with males being more aggressive than females. This effect was only significant for proactive aggression. A strong gender effect in the context of the TAP has been shown before (Dambacher et al., 2015). This is in line with a vast body of evidence supporting the notion that males tend to display more overt aggression than females especially in the domain of physical aggression as assessed by the TAP (Archer, 2004; Bjorkqvist, 1994; Eagly \& Steffen, 1986; Lagerspetz, Bjorkqvist, \& Peltonen, 1988).

Our findings seem plausible from a behavioural perspective and provide direct empirical support for the concept of an inverse relationship between response inhibition and aggression. However, although this study has sufficient statistical power (as revealed by post-hoc power analyses based on effect sizes by Dambacher et al., 2015), the here introduced bilateral tDCS protocols failed to reveal any effects on either response inhibition or proactive and reactive aggression. This means, that on a neural level, our tDCS findings fail to find evidence in favor of the hypothesized prefrontal cortical asymmetry in the domain of response inhibition and aggression. However, it is not possible to draw any clear conclusions as the 'absence of evidence' in brain stimulation research cannot simply be interpreted as 'evidence of absence' (DeGraaf \& Sack, 2011). The current results, which were obtained based on a clear hypothesis and sound methodology, can also give indications on the (in)efficacy of the brain stimulation parameters and stimulation sites chosen.

Our absence of tDCS effects are at odds with previous studies applying stimulation unilaterally and thereby modifying response inhibition or aggression: Anodal stimulation applied unilaterally to the right inferior frontal cortex enhanced the ability to inhibit responses in a stop signal paradigm (Jacobson et al., 2011). Applying cathodal stimulation unilaterally to the right dorsolateral prefrontal cortex was shown to impair response inhibition in a GNGT (Beeli et al., 2008). Finally, applying anodal stimulation unilaterally to the right dorsolateral prefrontal cortex was shown to reduce proactive aggression in males (Dambacher et al., 2015).

Our study, however, differed in several methodological aspects from these previous experiments. First, based on a concrete hypothesis derived from the expected inverse relationship between response inhibition and aggression and their opposing prefrontal lateralization, we opted for a bilateral stimulation protocol positioning both electrodes symmetrically over both hemispheres. This is opposed to the usage of a unilateral (or non-symmetrical) protocol, for which the return electrode would be positioned, for instance, over the orbit or mastoid of the hemisphere contralateral to the target site. We assumed that such a setup of enhancing activity in one hemisphere, while at the same time inhibiting activity in the region symmetric to the target site in the contralateral hemisphere, would directly manipulate the prefrontal cortical asymmetry underlying response inhibition and aggression. However, as of yet, no empirical evidence exists that supports the general validity of such bilateral tDCS montages, neither on behavioural nor neurophysiological level (Horvath et al., 2014; Horvath, 
Forte, \& Carter, 2015). It thus remains speculative whether bilateral tDCS protocols as described in the current study in fact induce the intended shifts in cortical balance between hemispheres.

Second, in accordance with existing imaging work localizing the main overlap of neural networks involved in response inhibition and aggression in inferior frontal cortex (Dambacher, Sack, Lobbestael, Arntz, Brugman, and Schuhmann, 2014a, 2014b), we chose this area as our tDCS target region. However, some previous studies demonstrating tDCS effects on response inhibition or aggression positioned the electrodes superior to our target sites within dorsolateral prefrontal cortex (Beeli et al., 2008; Hortensius et al., 2012; Dambacher et al., 2015).

Third, with $1.5 \mathrm{~mA}$ we stimulated with a rather low intensity compared to tDCS studies which observed a brain stimulation related effect (Hortensius et al., 2012; Dambacher et al., 2015).

As no study has specifically applied a bilateral tDCS protocol over inferior frontal cortex, the comparability of our study to previous findings is limited and our absence of tDCS effects could, thus, be due to any of those methodological differences. Hence, while the verdict on the validity of the prefrontal cortical asymmetry concept in the domain of response inhibition and aggression is still out, our current study clearly indicates that caution is warranted when conceptualizing the manipulation of hemispheric asymmetry by means of bilateral tDCS montages. 


\section{CHAPTER 5}

Anderson, C. A., \& Bushman, B. J. (2002). Human aggression. Annual Review of Psychology, 53, 27-51.

Anderson, C. A., Lindsay, J. J., \& Bushman, B. J. (1999). Research in the Psychological Laboratory: Truth or Triviality? Current Directions in Psychological Science, 8(1), 3-9.

Archer, J. (2004). Sex Differences in Aggression in Real-World Settings: A Meta-Analytic Review. Review of General Psychology, 8(4), 291-322.

Aron, A. R., Robbins, T. W., \& Poldrack, R. A. (2004). Inhibition and the right inferior frontal cortex. Trends in Cognitive Sciences, 8(4), 170-7.

Asahi, S., Okamoto, Y., Okada, G., Yamawaki, S., \& Yokota, N. (2004). Negative correlation between right prefrontal activity during response inhibition and impulsiveness: a fMRI study. European Archives of Psychiatry and Clinical Neuroscience, 254(4), 245-51.

Beeli, G., Casutt, G., Baumgartner, T., \& Jäncke, L. (2008). Modulating presence and impulsiveness by external stimulation of the brain. Behavioral and Brain Functions, 4, 33.

Bernstein, S., Richardson, D., \& Hammock, G. (1987). Convergent and discriminant validity of the Taylor and Buss measures of physical aggression. Aggressive Behavior, 13(1), 15-24.

Bjorkqvist, K. (1994). Sex differences in physical, verbal, and indirect aggression: A review of recent research. Sex Roles, 30(3-4), 177-188.

Carver, C. S., \& Harmon-Jones, E. (2009). Anger is an approach-related affect: evidence and implications. Psychological Bulletin, 135(2), 183-204.

Chambers, C. D., Bellgrove, M. A., Stokes, M. G., Henderson, T. R., Garavan, H., Robertson, I. H., ... Mattingley, J. B. (2006). Executive "brake failure" following deactivation of human frontal lobe. Journal of Cognitive Neuroscience, 18(3), 444-55.

Chambers, C. D., Bellgrove, M. A., Gould, I. C., English, T., Garavan, H., McNaught, E., ... Mattingley, J. B. (2007). Dissociable mechanisms of cognitive control in prefrontal and premotor cortex. Journal of Neurophysiology, 98(6), 3638-47.

d'Alfonso, A. A., van Honk, J., Hermans, E., Postma, A., \& de Haan, E. H. (2000). Laterality effects in selective attention to threat after repetitive transcranial magnetic stimulation at the prefrontal cortex in female subjects. Neuroscience Letters, 280(3), 195-8.

Dambacher, F., Sack, A. T., Lobbestael, J., Arntz, A., Brugman, S., \& Schuhmann, T. (2014a). A network approach to response inhibition: dissociating functional connectivity of neural components involved in action restraint and action cancellation. The European Journal of Neuroscience, 39(5), 821-31.

Dambacher, F., Sack, A. T., Lobbestael, J., Arntz, A., Brugman, S., \& Schuhmann, T. (2014b). Out of control Evidence for anterior insula involvement in motor impulsivity and reactive aggression. Social Cognitive and Affective Neuroscience, in print, doi: 10.1093/scan/nsu077.

Dambacher, F., Sack, A. T., Lobbestael, J., Arntz, A., Brugmann, S., \& Schuhmann, T. (2014c). The role of right prefrontal and medial cortex in response inhibition: interfering with action restraint and action cancellation using transcranial magnetic brain stimulation. Journal of Cognitive Neuroscience, 26(8), 1775-84.

Dambacher, F., Schuhmann, T., Lobbestael, J., Arntz, A., Brugman, S., \& Sack, A. T. (2015). Reducing proactive aggression through Non-Invasive Brain Stimulation. Social cognitive and affective Neuroscience, in print.

De Graaf, T. A., \& Sack, A. T. (2011). Null results in TMS: from absence of evidence to evidence of absence. Neuroscience and Biobehavioral Reviews, 35(3), 871-7.

Eagle, D. M., Bari, A., \& Robbins, T. W. (2008). The neuropsychopharmacology of action inhibition: crossspecies translation of the stop-signal and go/no-go tasks. Psychopharmacology, 199(3), 439-56.

Eagly, A. H., \& Steffen, V. J. (1986). Gender and aggressive behavior: a meta-analytic review of the social psychological literature. Psychological Bulletin, 100(3), 309-30.

Faul, F., Erdfelder, E., Lang, A.-G., \& Buchner, A. (2007). G*Power 3: a flexible statistical power analysis program for the social, behavioral, and biomedical sciences. Behavior Research Methods, 39(2), 17591.

Giancola, P. R., \& Parrott, D. J. (2008). Further evidence for the validity of the Taylor Aggression Paradigm. Aggressive Behavior, 34(2), 214-29. 
Giancola, P. R., \& Zeichner, A. (1995). Construct validity of a competitive reaction-time aggression paradigm. Aggressive Behavior, 21(3), 199-204.

Harmon-Jones, E. (2004). Contributions from research on anger and cognitive dissonance to understanding the motivational functions of asymmetrical frontal brain activity. Biological Psychology, 67(1-2), 51-76.

Harmon-Jones, E., \& Allen, J. J. (1998). Anger and frontal brain activity: EEG asymmetry consistent with approach motivation despite negative affective valence. Journal of Personality and Social Psychology, 74(5), 1310-6.

Harmon-Jones, E., \& Sigelman, J. (2001). State anger and prefrontal brain activity: evidence that insultrelated relative left-prefrontal activation is associated with experienced anger and aggression. Journal of Personality and Social Psychology, 80(5), 797-803.

Hoaken, P. N. S., Shaughnessy, V. K., \& Pihl, R. O. (2003). Executive cognitive functioning and aggression: Is it an issue of impulsivity? Aggressive Behavior, 29(1), 15-30.

Horn, N. R., Dolan, M., Elliott, R., Deakin, J. F. W., \& Woodruff, P. W. R. (2003). Response inhibition and impulsivity: an fMRI study. Neuropsychologia, 41(14), 1959-66.

Hortensius, R., Schutter, D. J. L. G., \& Harmon-Jones, E. (2012). When anger leads to aggression: induction of relative left frontal cortical activity with transcranial direct current stimulation increases the angeraggression relationship. Social Cognitive and Affective Neuroscience, 7(3), 342-7.

Horvath, J. C., Forte, J. D., \& Carter, O. (2014). Evidence that transcranial direct current stimulation (tDCS) Generates little-to-no reliable neurophysiologic effect beyond MEP amplitude modulation in healthy Human subjects: A systematic review. Neuropsychologia, 66, 213-236.

Horvath, J. C., Forte, J. D., \& Carter, O. (2015). Quantitative Review Finds No Evidence of Cognitive Effects in Healthy Populations from Single-Session Transcranial Direct Current Stimulation (tDCS). Brain Stimulation, in print, doi:10.1016/j.brs.2015.01.400.

Jacobson, L., Javitt, D. C., \& Lavidor, M. (2011). Activation of inhibition: diminishing impulsive behavior by direct current stimulation over the inferior frontal gyrus. Journal of Cognitive Neuroscience, 23(11), 3380-7.

Lagerspetz, K. M. J., Bjorkqvist, K., \& Peltonen, T. (1988). Is Indirect Aggression Typical of Females ? Gender Differences in Aggressiveness in 1- to 12-year-old children. Aggressive Behavior, 14, 403-414.

Logan, G. D., Schachar, R. J., \& Tannock, R. (1997). Impulsivity and Inhibitory Control. Psychological Science, $8(1), 60-64$.

Pawliczek, C. M., Derntl, B., Kellermann, T., Kohn, N., Gur, R. C., \& Habel, U. (2013). Inhibitory control and trait aggression: Neural and behavioral insights using the emotional stop signal task. Neurolmage, 79C, 264-274.

Poulin, F., \& Boivin, M. (2000). Reactive and proactive aggression: Evidence of a two-factor model. Psychological Assessment, 12(2), 115-122.

Raine, A., Dodge, K., Loeber, R., Gatzke-Kopp, L., Lynam, D., Reynolds, C., ... Liu, J. (2006). The ReactiveProactive Aggression Questionnaire: Differential Correlates of Reactive and Proactive Aggression in Adolescent Boys. Aggressive Behavior, 32(2), 159-171.

Taylor, S. P. (1967). Aggressive behavior and physiological arousal as a function of provocation and the tendency to inhibit aggression. Journal of Personality, 35(2), 297-310.

Van Honk, J., \& Schutter, D. J. L. G. (2006). From affective valence to motivational direction: the frontal asymmetry of emotion revised. Psychological Science, 17(11), 963-5.

Verbruggen, F., Aron, A. R., Stevens, M. A., \& Chambers, C. D. (2010). Theta burst stimulation dissociates attention and action updating in human inferior frontal cortex. Academy of Sciences of the United States of America, 107(31), 13966-71. 



\section{CHAPTER 6}

\section{The role of the insular cortex in retaliation}

"The sad truth is that most evil is done by people who never make up their minds to be good or evil."

- Hannah Arendt, The Life of the Mind, 1978 
Based on: Dambacher, F., Schuhmann, T., Lobbestael, J., Arntz, A., Brugman, S., \& Sack, A. T. (2015). The role of the insular cortex in retaliation. submitted

We thank Ulrike Krämer, Frederike Beyer, Armin Heinecke, Martijn Stroom, Martijn van Teffelen, Clara Bernier, Oana Gurau, and Thomas C. Emmerling for their help. 


\section{ABSTRACT}

The insular cortex has consistently been associated with various aspects of emotion processing and social behaviour, including anger processing and overt aggression. Aggression research distinguishes proactive or instrumental aggression from retaliation, i.e. aggression in response to provocation. Here, we investigated the specific role of the insular cortex during retaliation, employing a controlled behavioural aggression paradigm implementing different levels of provocation. Fifteen healthy male volunteers underwent whole brain functional magnetic resonance imaging (fMRI) to identify brain regions involved in interaction with either a provoking or a non-provoking opponent. FMRI group analyses were complemented by examining the parametric modulations of brain activity related to the individual level of displayed aggression. These analyses identified a hemispheric lateralization as well as an anatomical segregation of insular cortex with specifically the left posterior part being involved in retaliation. The left-lateralization of insular activity during retaliation is in accordance with evidence from electro-physiological studies, suggesting left-lateralized fronto-cortical dominance during anger processing and aggressive acts. The posterior localization of insular activity, on the other hand, suggests a spatial segregation within insular cortex with particularly the posterior part being involved in the processing of emotions that trigger intense bodily sensations and immediate action tendencies. 


\section{CHAPTER 6}

Aggression is defined as the intentional infliction of harm to another being (Anderson \& Bushman, 2002). Different forms of aggression are distinguished; proactive aggression refers to using aggression in an instrumental, goal-oriented way, whereas reactive aggression refers to retaliation, i.e. aggressive actions triggered by preceding provocation (Poulin \& Boivin, 2000; Raine et al., 2006).

Aggression and retaliation are complex social behaviours and their scientific assessments rely on social interaction paradigms that do not only measure the perception of - or attention to - specific social emotional cues, but also their behavioural consequences in an experimental setup. Ideally, such paradigms allow quantifying different levels of aggressive behaviour within provocative and non-provocative interactions in a controlled way. One of the most widely used and validated behavioural aggression paradigms fulfilling these requirements is the Taylor Aggression Paradigm (TAP), which also proofed to be feasible in an neuroimaging environment (Beyer, Münte, Erdmann, \& Krämer, 2014; Beyer, Münte, Göttlich, \& Krämer, 2014; Brunnlieb, Münte, Krämer, Tempelmann, \& Heldmann, 2013; Dambacher et al., 2014; Krämer, Jansma, Tempelmann, \& Münte, 2007; Lotze, Veit, Anders, \& Birbaumer, 2007). The TAP is set up as a reaction time game between two or more opponents in which the winner can administer an aversive feedback stimulus of variable intensity to the opponent; therewith it measures aggressive behaviour within direct social interactions in a controlled way. Several neuroimaging studies have aimed to identify the neural activations induced by the TAP, reporting predominantly prefrontal regions, parietal cortex, basal ganglia, thalamus (Krämer et al., 2007), and dorsal medial prefrontal cortex (Lotze et al., 2007) involvement during the interaction between opponents. Prefrontal regions, striatum, and other parts of the reward network (Krämer et al., 2007) were activated when winning (versus loosing) against the opponent. Furthermore, the insular cortex was especially associated with aggressive behavior during this social interaction game (Dambacher et al., 2014; Krämer et al., 2007). However, insular cortex involvement has also been reported in many other contexts including the processing of positive emotions, action inhibition, mindfulness, and interoception (Craig, 2009), questioning any functional specificity of its involvement.

The seemingly rather general involvement of insular cortex in a variety of emotional and cognitive paradigms led to the development of models which could potentially assign different functions to different parts of the insular cortex. For instance, a segregation along a posterior-to-anterior gradient representing the progressive integration of bodily feelings has been proposed (Craig, 2009): Whereas acute emotions or interoceptive components might be represented in the posterior parts of the insular cortex, the anterior parts seem to code for more abstract and highly integrated constructs. This suggests that - opposite to the anterior insular cortex - the posterior insular cortex is involved in the processing of emotions triggering intense bodily sensation and immediate action tendencies. 
Besides its segregation, the lateralization of insular activation is yet to be fully understood. Previous work suggested that the left hemisphere - as opposed to the right - generally might be more involved in approach related motivational states such as anger processing and aggression (Harmon-Jones, 2004; van Honk \& Schutter, 2006). It remains to be answered, however, whether this assumption holds true not only regarding overall fronto-cortical asymmetry, but also with respect to specific brain regions such as the insular cortex.

Following this line of argumentation, we particularly expect the posterior parts of the insular cortex to be involved in retaliation during which intense bodily sensations, action-oriented emotional content, and immediate behavioural responses are mobilized. Furthermore, this activation is expected to be left-lateralized as retaliation is closely related to anger processing and approach motivation. The here presented study tests this hypothesis by assessing the parametric modulations of brain activity underlying retaliation in a controlled aggression paradigm during whole brain fMRI.

\section{MATERIALS AND METHODS}

Please note that the here reported analyses are based on the same data set used in a previously reported study which compared the neural correlates of reactive aggression with regions involved in motor impulsivity (measured with a go-/nogo task; Dambacher et al., 2014).

Participants. Eighteen male university students volunteered, gave their written informed consent, and were paid for participating. A screening ensured that none of the participants had a previous history of neurological or psychiatric disorders. Data of two participants were excluded from the analyses as they did not follow the instructions of the experimenter. Another participant was excluded, since he did not show any reaction to provocation and always chose low reactions. Data of fifteen participants were included in further analyses (mean age $=22.33$; SD $=2.35$ ). The study was approved by the local Ethical Committee of the Faculty of Psychology and Neuroscience at Maastricht University.

Taylor Aggression Paradigm (TAP). Introduced in its first version by Taylor in 1967 (Taylor, 1967), the TAP has become a common tool in behavioural aggression research and has also proven itself the most adaptable option for brain imaging studies (Beyer, Münte, Göttlich, \& Krämer, 2014; Dambacher et al., 2014; Krämer, Jansma, Tempelmann, \& Münte, 2007; Lotze, Veit, Anders, \& Birbaumer, 2007). The task is set up as a competitive reaction time game between two or more opponents. The players give each other feedback after each reaction time trial. During the task, aggressive behaviour is measured by recording the severity level of the feedback or "punishment" participants assign to their virtual opponents. The level of provocation can be manipulated such that an opponent can choose a more or less aversive feedback for the other 
player. Whenever a player loses a reaction time trial, he is presented with the feedback chosen by the opponent. The Taylor Aggression Paradigm has shown to be high in construct, internal, discriminant as well as external validity (Anderson, Lindsay, \& Bushman, 1999; Bernstein, Richardson, \& Hammock, 1987; Giancola \& Zeichner, 1995).

During recruitment, participants were led to believe that the experiment investigated the impact of human feedback on reaction time performance. They were informed about playing a reaction time game (TAP) against two other participants. Before entering the scanner, the participant and the two opponents (collaborators of the experimenters) were introduced. The experimenter's collaborators were trained beforehand and acted according to a script in order to ensure equal treatment of all participants. Throughout the entire scan, the players communicated verbally via intercom. Immediately after completion of the experiment, an exit interview was administered to ensure that participants were fully deceived by the experimental setup. Upon completion of the study, a written debriefing was provided.

In the implementation of the TAP employed in this study (see also Dambacher et al., 2014, Fig.1), the participant played reaction time trials against one of two alleged opponents randomly. These opponents were collaborators of the experimenter and merely acted in their role as participants. Participants were told that whoever reacted faster to a target stimulus, won the trial. In cases that participants were slower than the alleged opponent, they were presented with an aversive feedback noise. At the beginning of each trial the volume of this noise was chosen on an 8-point scale. Feedback noises were adjusted to the individual threshold of endurability while running a functional sequence for each participant. No noises above 100 decibel were administered to ensure that the hearing was not compromised. Participants randomly played against each of the putative opponents in $50 \%$ of the trials. One opponent always selected soft feedback noises (from 1 to 4; non-provoking opponent), while the other selected loud feedback noises (from 4 to 8; provoking opponent). Participants randomly won (and lost) in 50\% of trials per opponent. Trials in which reaction times exceeded $500 \mathrm{msec}$ always were losing trials. This ensured a realistic sensation of competing with a human opponent. 


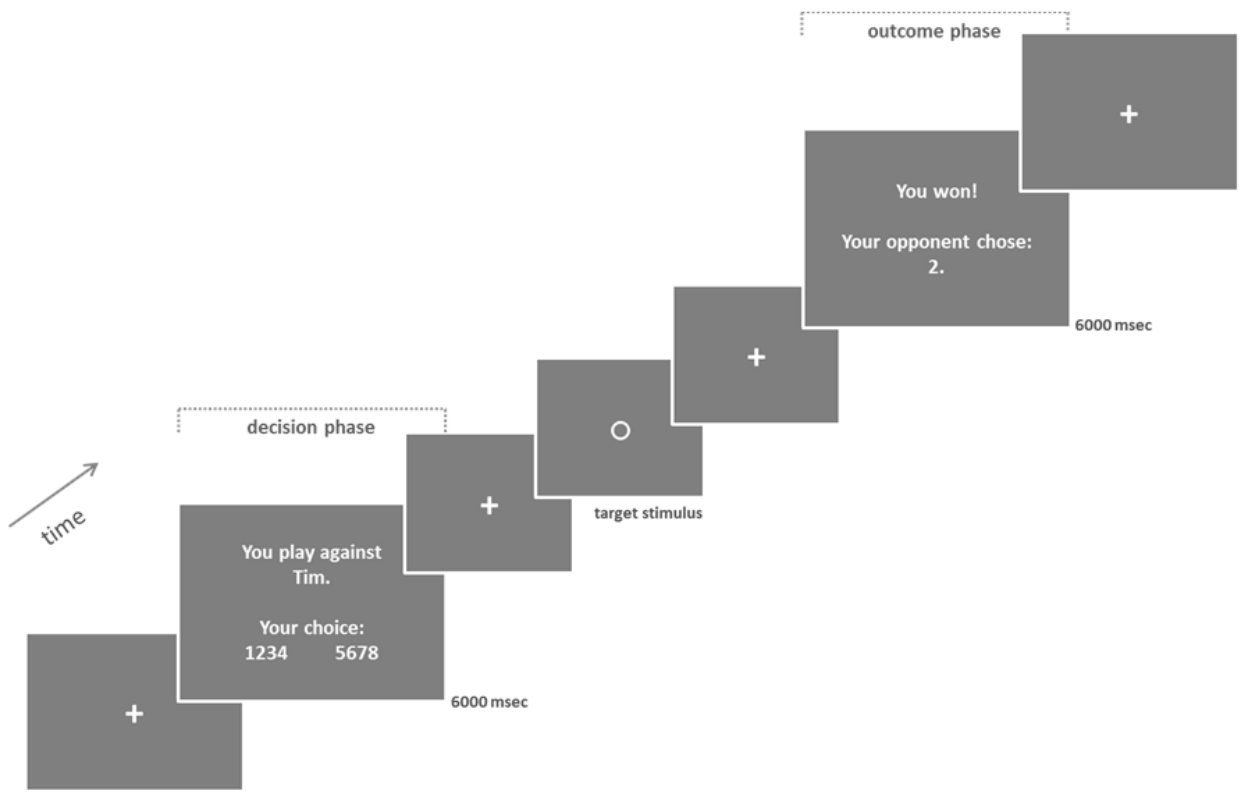

Fig. 1. Taylor Aggression Paradigm (TAP). Adapted from Dambacher et al., 2014. During the decision phase, participants were presented with a screen that informed them against whom they were playing in this trial (in this case "Tim") and asked to choose the feedback noise level that should be administered to this opponent in case the opponent lost ("12345678"). During the outcome phase, participants were informed on whether or not they won and what feedback noise level the particular opponent had chosen for this trial.

Each trial of 27000 msec consisted of a decision phase (6000 msec), the actual reaction time game (jittered between 4500 and $7500 \mathrm{msec}$ ), and an outcome phase (6000 $\mathrm{msec}$ ). A jittered resting period followed. During the decision phase, participants were presented with a screen that informed them against whom they were playing in this particular trial ("Rob" or "Tim") and asked to choose the volume of the feedback they wanted to administer to this opponent in case he would lose. The actual reactive aggressive behaviour was measured during the decision phase of the TAP. During the outcome phase, participants were informed about whether or not they lost in this particular trial and which feedback noise levels the particular opponent chose for this trial. Whenever they lost, they were presented with this noise at the end of the outcome phase.

Stimuli were presented in white (RGB 255/255/255; Arial pt 24) on a grey background (RGB 125/125/125). Participants performed 3 runs of the TAP including 28 trials (14 trials per opponent) each, leading to a total of 84 trials (42 trials per opponent). Stimuli were presented using Presentation software (Neurobehavioural Systems, Inc., Albany, USA). Behavioural statistical analyses were performed using SPSS19 (IBM Statistics, USA). 


\section{CHAPTER 6}

Technical details, fMRI acquisition and analysis. (see also Dambacher et al., 2014) Stimulus material was presented using an LCD projector (Panasonic, No PT-EZ57OEL) mounted onto a frosted screen, positioned at rear of the scanner bore. Responses were registered with a standard MR compatible button box (Current Designs, 8-button response device, HHSC-2x4-C, Philadelphia, USA).

Images were acquired with a 3 Tesla Siemens Prisma scanner. Structural (high resolution T1-weighted MPRAGE; isotropic voxel resolution 1x1x1 $\mathrm{mm}^{3} ; 192$ sagittal slices) and functional whole-brain (Gradient-Echo-EPI-sequence; TR=1500msec; TE $=26 \mathrm{msec}$; FOV $=224 \mathrm{~mm}$; flip angle $=73^{\circ}$; matrix $=64 \times 64$; distance factor $=20 \%$; 478 volumes per run for the GNGT, 512 volumes per run for the TAP) scans were recorded. Twenty-eight oblique transversal slices of $3.5 \times 3.5 \times 3.5 \mathrm{~mm}$ voxels were obtained. Slices were tilted $30^{\circ}$ relatively to the anterior-posterior commissure plane to avoid signal dropout in frontal areas (Deichmann, Gottfried, Hutton, \& Turner, 2003).

FMRI data were analyzed with Brain Voyager QX (Brain Innovation BV, Maastricht, The Netherlands). Preprocessing included 3D-motion-correction (trilinear / sinc interpolation and intra-session alignment to the first functional volume recorded after the individual structural scan), cubic spline slice scan time correction, and the application of a temporal high pass filter (general linear model (GLM) with Fourier basis set of 3 cycles sine/cosine per run plus linear trend removal). Images were co-registered to the individual anatomical data sets and normalized to Talairach stereotaxic space (Talairach \& Tournoux, 1988). Volume time courses were spatially smoothed (6mm full width half maximum Gaussian kernel).

The first three trials per opponent were excluded to restrict the analyses to the trials in which participants were familiar with the distinct behavioural pattern of the two opponents (i.e. provoking versus non-provoking).

Random effects group analyses. A GLM was defined to analyze the behaviour displayed during the decision and the outcome phase in the TAP. For these analyses, the feedback given by the participants was grouped into low (level 1-3), middle (4 and 5), and high (level 6-8) punishment.

The following conditions were included as predictors for the decision phase (for phases of TAP see figure 1): participant chooses high punishment for the provoking opponent, participant chooses low punishment for the non-provoking opponent. Some participants rarely or never chose a low or middle punishment for the provoking opponent. Furthermore, not every participant chose a middle or high punishment for the non-provoking opponent. These conditions could therefore not be taken into account on the level of group analyses.

The following conditions were included as predictors for the outcome phase (for phases of TAP see figure 1): all win trials, all loose trials, win trials against provoking opponent, loose trials against provoking opponent, win trials against non-provoking opponent, loose trials against non-provoking opponent. 
To reduce error variance, one noise regressor consisting of the first eigenvariate time series from cerebrospinal fluid regions and motion artefacts were included into the analyses as covariates. Statistical maps were created using a threshold of $p<.001$ corrected for multiple comparisons by means of cluster threshold level estimation (1000 Monte Carlo simulation iterations; Forman et al., 1995).

Analyses of parametric modulations. For these analyses, the feedback given by the participants was treated as a continuous linear variable (from 1 to 8). For the decision phase, a main and a parametric predictor for interaction with the provoking opponent and the non-provoking opponent were defined. For the decision phase, a main and a parametric predictor for winning and losing against the provoking opponent and the non-provoking opponent were defined. Parametric predictors were weighted on a single trial bases according to the behaviour the participant displayed (the punishment chosen) in the respective trial.

In order to examine which brain regions were modulated by the chosen punishment, the conjunction of the main and the parametric effect for each specific condition (decision and outcome phase) was inspected.

Statistical maps were created using a threshold of $p<.01$ corrected for multiple comparisons by means of cluster threshold level estimation (1000 Monte Carlo simulation iterations; Forman et al., 1995).

\section{RESULTS}

Behavioural data. (previously reported in Dambacher et al., 2014). The average feedback (i.e. punishment by mean of aversive noise) selected by the participants for the opponents was of medium intensity (MEAN $=3.54$; SD $=.04$ ). A significantly higher feedback was chosen for the provoking compared to the non-provoking opponent (provoking opponent: MEAN $=4.52, \mathrm{SD}=.64$; non-provoking opponent: $\mathrm{MEAN}=2.56$, $S D=1.17 ; t=4.59, p=<.001)$. During the exit interview at the end of the experiment no participant reported doubting the proposed purpose of the study and all fifteen participants reported that they perceived one opponent as more provocative than the other. Twelve participants explicitly reported that they adapted their reaction to that perception. Similarly, an analysis of variance with repeated measures revealed a significant interaction effect of high, middle, and low feedback level chosen by the participants and the type of opponent $(F=19.5, p<.001)$. Thereby, high punishment was chosen more frequently for the provoking than the non-provoking opponent, low punishment was chosen more often for the non-provoking than for the provoking opponent. 


\section{CHAPTER 6}

Tab. 1. Talairach coordinates. Center of gravity, number of significant voxels per cluster, and maximum statistical t-value; clusters are labeled according to Talairach Client, Lancaster et al. $(1997,2000)$.

\begin{tabular}{|c|c|c|c|c|c|}
\hline \multirow[b]{2}{*}{ Region } & \multicolumn{3}{|c|}{ Talairach coordinates } & \multicolumn{2}{|l|}{ Size } \\
\hline & $x$ & $y$ & $z$ & voxel & $t$ \\
\hline
\end{tabular}

\section{RFX GLM}

Aggressive reaction to provoking opponent > non aggressive reaction to non-provoking opponent

$\begin{array}{lcccccc}\text { Anterior insular cortex } & \mathrm{R} & 27 & 21 & 10 & 1126 & 6.43 \\ \text { Anterior insular cortex connected } & \mathrm{L} & -17 & -3 & 9 & 13798 & 7.41 \\ \text { Insular cortex connected } & \mathrm{L} & -17 & -3 & 9 & 13798 & 7.41 \\ \text { Putamen / globus pallidus connected } & \mathrm{R} & -17 & -3 & 9 & 13798 & 7.41 \\ \text { Putamen / globus pallidus connected } & \mathrm{L} & -17 & -3 & 9 & 13798 & 7.41 \\ \text { Thalamus connected } & \mathrm{L} & -17 & -3 & 9 & 13798 & 7.41 \\ \text { Caudate connected } & \mathrm{L} & -17 & -3 & 9 & 13798 & 7.41 \\ \text { Parietal lobe, postcentral gyrus } & \mathrm{L} & -38 & -34 & 53 & 30935 & 9.96 \\ \text { Frontal Lobe, paracentral lobe } & \mathrm{L} & -4 & -11 & 47 & 2900 & 6.05 \\ \text { Cerebellum } & \mathrm{R} & 12 & -65 & 18 & 7471 & 7.87\end{array}$

Non aggressive reaction to non-provoking opponent > aggressive reaction to provoking opponent

Parietal lobe, postcentral gyrus

Superior temporal gyrus

Won > lost

Superior frontal gyrus

Middle frontal gyrus

Middle frontal gyrus

Middle frontal gyrus

Inferior parietal lobe, postcentral gyrus

Inferior parietal lobe, postcentral gyrus

Parietal lobe, precuneus

Striatum

Striatum

\section{Lost > won}

Superior temporal gyrus

Superior temporal gyrus

Limbic lobe, parahippocampal gyrus

Limbic lobe, parahippocampal gyrus

R $\quad 36$

R 53

$-31$

$\begin{array}{lll}4 & -7 & 348\end{array}$

6.52

6.04

$\begin{array}{lrrrcr}\mathrm{R} & 21 & 56 & 18 & 1056 & 5.87 \\ \mathrm{~L} & -44 & 54 & 7 & 449 & 5.26 \\ \mathrm{R} & 29 & 8 & 51 & 16489 & 11.67 \\ \mathrm{~L} & -33 & 6 & 51 & 17993 & 8.20 \\ \mathrm{R} & 38 & -56 & 37 & 17931 & 8.07 \\ \mathrm{~L} & -39 & -55 & 37 & 18740 & 8.49 \\ \mathrm{~L} & 0 & -63 & 34 & 721 & 5.26 \\ \mathrm{R} & 11 & 8 & 3 & 761 & 5.77 \\ \mathrm{~L} & -16 & 10 & 3 & 1865 & 8.07\end{array}$

$\begin{array}{rrrrlr}\mathrm{R} & 48 & -15 & 8 & 35116 & 12.37 \\ \mathrm{~L} & -49 & -20 & 8 & 18628 & 9.87 \\ \mathrm{R} & 18 & -50 & -2 & 3383 & 7.57 \\ \mathrm{~L} & -20 & -55 & -1 & 1552 & 6.04\end{array}$

Won against the provoking opponent $>$ won against the non-provoking opponent No significant modulation detected

Won against the non-provoking opponent > won against the provoking opponent No significant modulation detected

Lost against the provoking opponent > lost against the non-provoking opponent No significant modulation detected 


\begin{tabular}{|c|c|c|c|c|c|c|}
\hline \multirow{2}{*}{ Region } & & \multicolumn{3}{|c|}{ Talairach coordinates } & \multicolumn{2}{|l|}{ Size } \\
\hline & & $x$ & $y$ & $z$ & voxel & $t$ \\
\hline \multicolumn{7}{|c|}{ Lost against the non-provoking opponent > lost against the provoking opponent } \\
\hline Parietal lobe, around postcentral gyrus & $\mathrm{R}$ & 35 & -32 & 52 & 2685 & 7.64 \\
\hline Parietal lobe, around postcentral gyrus & $\mathrm{L}$ & -11 & -41 & 68 & 1508 & \\
\hline Middle temporal gyrus & $\mathrm{L}$ & -41 & -64 & 26 & 665 & 6.13 \\
\hline \multicolumn{7}{|l|}{ PARAMETRIC MODULATIONS } \\
\hline \multicolumn{7}{|l|}{ Retaliation independent of opponent } \\
\hline Inferior parietal lobe, pre- and postcentral gyrus & $\mathrm{L}$ & -38 & -36 & 51 & 29148 & 6.16 \\
\hline Cerebellum & $\mathrm{R}$ & 14 & -49 & -20 & 1350 & 3.98 \\
\hline \multicolumn{7}{|l|}{ Retaliation interacting with provoking opponent } \\
\hline Inferior parietal lobe, pre- and postcentral gyrus & $\mathrm{L}$ & -36 & -34 & 56 & 17268 & 6.14 \\
\hline Cerebellum & $\mathrm{R}$ & 11 & -53 & -19 & 1703 & 4.79 \\
\hline Insular cortex & $\mathrm{L}$ & -39 & -8 & 15 & 1562 & 6.13 \\
\hline \multicolumn{7}{|l|}{ Retaliation interacting with non-provoking opponent } \\
\hline Inferior parietal lobe, pre- and postcentral gyrus & $\mathrm{L}$ & -41 & -38 & 47 & 10864 & 4.21 \\
\hline \multicolumn{7}{|l|}{ Won } \\
\hline \multicolumn{7}{|l|}{ No significant modulation detected } \\
\hline \multicolumn{7}{|l|}{ Won against provoking opponent } \\
\hline \multicolumn{7}{|l|}{ No significant modulation detected } \\
\hline \multicolumn{7}{|l|}{ Won against non-provoking opponent } \\
\hline No significant modulation detected & & & & & & \\
\hline
\end{tabular}

Random effects group analyses. Talairach coordinates of the brain regions showing increased activation associated with the investigated contrasts are reported in table 1 (reported are the center of gravity, the number of significant voxels per cluster, and the maximum statistical t-value; cluster are labeled according to Talairach Client, Lancaster et al., 1997, 2000). Statistical maps of random effects group analyses are depicted in figure 2 for the decision and the outcome phase.

When contrasting trials in which participants gave a high punishment to the provoking opponent with trials in which the participant gave a low punishment to the nonprovoking opponent (provocation > no provocation; only contrast previously reported in Dambacher et al., 2014), increased activation in bilateral insular cortex, left parietal lobe, left-lateralized motor regions, the left frontal lobe, and cerebellum was observed. Furthermore, several subcortical regions (i.e., the right and left putamen/globus pallidus, left-lateralized thalamic regions and caudate) showed significant activation change associated to this contrast (Fig.2, Tab.1). The only significant activation change associated with giving a low punishment to the non-provoking opponent (no provocation > provocation) was observed in the right parietal lobe close to the postcentral gyrus and the right superior temporal gyrus. 
During winning (all won trials > all lost trials), strong significant bilateral activation in the right superior frontal gyrus, the middle frontal gyri, the left precuneus, the inferior parietal lobes, and the striatum was observed. During loosing (all lost trials $>$ all won trials), strong significant bilateral activation in the superior temporal gyri and the parahippocampal gyri was observed. No differences in brain activity could be detected when winning against the provoking opponent as opposed to winning against the nonprovoking opponent (won trials against the provoking opponent > won trials against the non-provoking opponent; won trials against the non-provoking opponent $>$ won trials against the provoking opponent). When participants lost to the non-provoking opponent versus to the provoking opponent (lost trials against the non-provoking opponent > lost trials against the provoking opponent), significant bilateral activation in the parietal lobes around the postcentral gyri and the left middle temporal gyrus could be detected. When participants lost to the provoking opponent versus to the non-provoking opponent (lost trials against the provoking opponent > lost trials against the non-provoking opponent), no significant differential brain activity could be detected. 
A) aggressive reaction to provoking opponent $>$ non aggressive reaction to non-provoking opponent
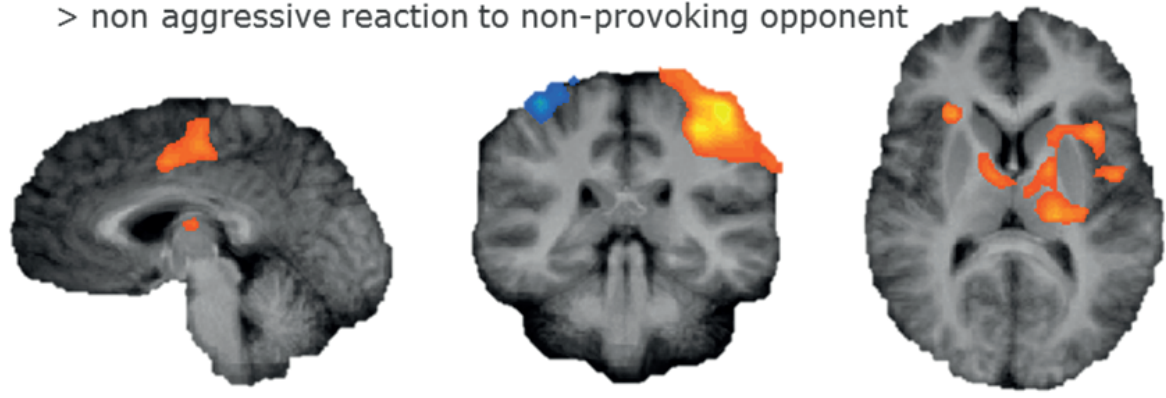

B) all won trials > all lost trials
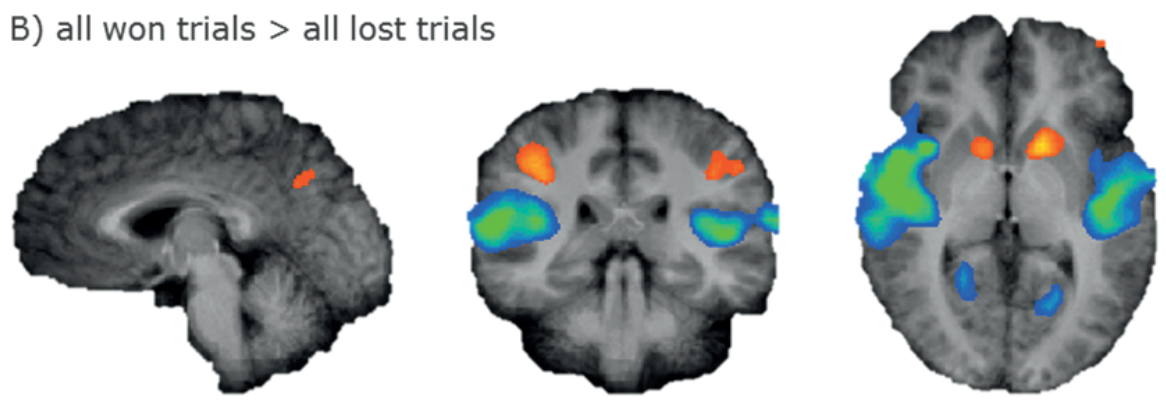

C) lost against non-provoking opponent $>$ lost against provoking opponent
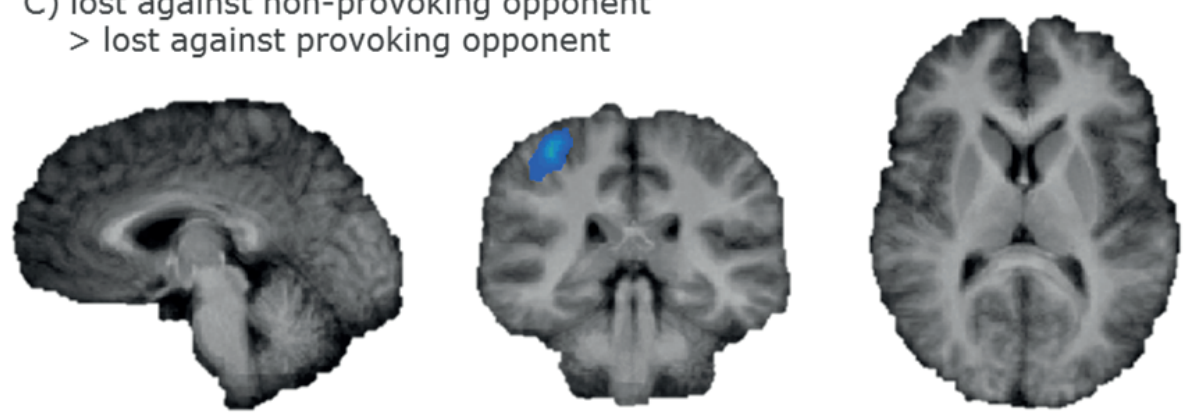

Fig. 2. Random effects group analyses. Neural activation for the specified contrasts containing significant activation during the decision phase $(\mathrm{A})$ and the outcome phase $(\mathrm{B}, \mathrm{C})$ of the Taylor Aggression Paradigm. Statistical Maps: orange > blue, $\mathrm{N}=15, \mathrm{p}<.001$, Cluster Threshold level corrected, radiological convention.

Parametric modulations. Talairach coordinates of the brain regions showing parametric modulations according to the displayed behaviour are reported in table 1 . Statistical maps of parametric modulations are depicted in figure 3. 


\section{CHAPTER 6}

In order to identify brain regions that modulate their activity according to the displayed behaviour (volume of the punishment chosen for the opponent defined on an 8-point scale), we conducted additional analyses in which the predictors were weighted according to this behaviour. During retaliation, activity in the left pre- and post-central gyri, thus motor activation associated to the movement of the right hand was most prominent (main effect of retaliation against provoking opponent, nonprovoking opponent, or both ${ }^{\wedge}$ parametric effect of retaliation against provoking opponent, non-provoking opponent, or both; conjunction). When interacting with the provoking opponent, the left posterior insular cortex was modulated by the intensity of punishment that was chosen for the provoking opponent (main effect of retaliation against provoking opponent ${ }^{\wedge}$ parametric effect of retaliation against provoking opponent; conjunction). This could not be observed for the interaction with the nonprovoking opponent. Finally, activation in the right cerebellum was parametrically modulated by the given punishment when interacting with both the non-provoking and the provoking opponent (main effect of retaliation against provoking opponent or both opponents ${ }^{\wedge}$ parametric effect of retaliation against provoking opponent or both opponents; conjunction). During the outcome phase no significant parametric modulations due to the punishment chosen by the participants could be observed (main effect all win trials, win trials against provoking opponent, or win trials against non-provoking opponent $\wedge$ parametric effect all win trials, win trials against provoking opponent, or win trials against non-provoking opponent; conjunction).

During the outcome phase no significant parametric modulations due to the punishment chosen by the participants could be observed (main effect all win trials, win trials against provoking opponent, or win trials against non-provoking opponent ${ }^{\wedge}$ parametric effect all win trials, win trials against provoking opponent, or win trials against nonprovoking opponent; conjunction). 

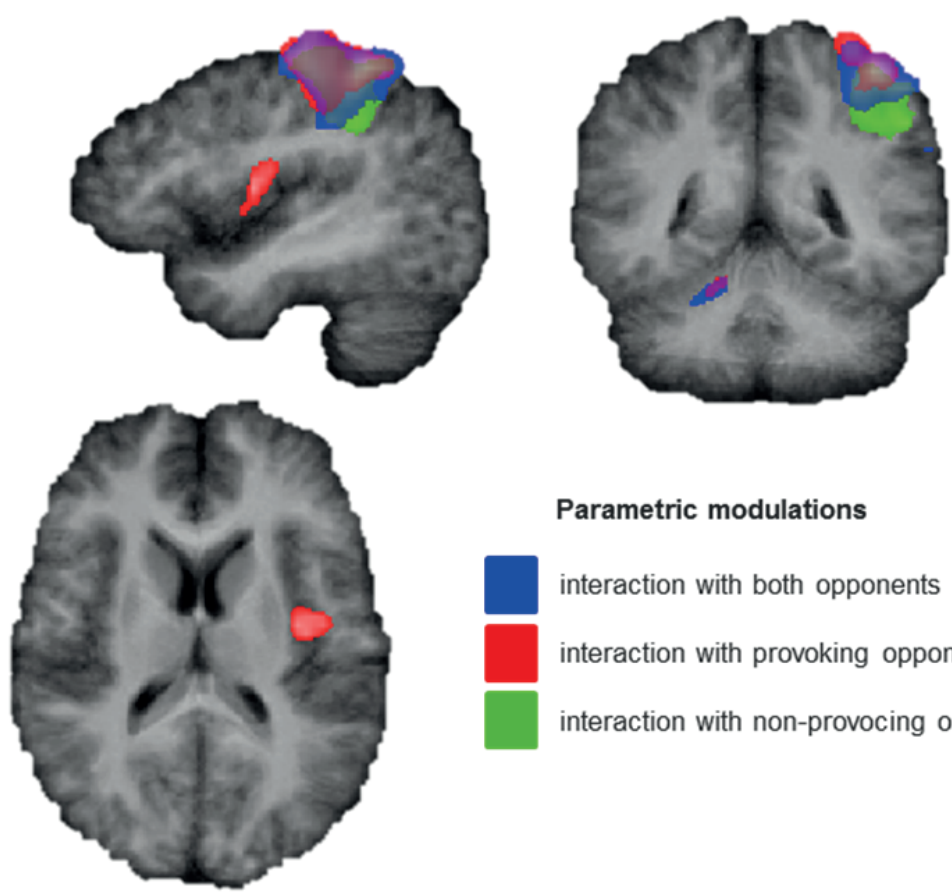

Parametric modulations

interaction with both opponents

interaction with provoking opponent

interaction with non-provocing opponent

Fig. 3. Parametric modulations. Regions modulating their activity parametrically according to the displayed behaviour, when interacting with both (blue), the provoking (red), or the non-provoking opponent (green) during the Taylor Aggression Paradigm. Statistical Maps: N=15, $<<.01$, Cluster Threshold level corrected, radiological convention.

\section{DISCUSSION}

The current study investigated the role of the insular cortex during retaliation and revealed that the left posterior insular cortex is specifically activated when interacting with a provoking - as opposed to a non-provoking - opponent.

The role, segregation, and lateralization of insular activation during retaliation. During an aggressive reaction to provocation bilateral insular cortex and basal ganglia were mainly activated. This is in line with previous work associating insular cortex with aggression and the processing of negative emotions (Krämer et al., 2007; Sanfey, Rilling, Aronson, Nystrom, \& Cohen, 2003). Our study provides direct quantitative support for the notion that the insular cortex is playing a crucial role in aggressive behaviour: We demonstrate that exclusively insular cortex activity is parametrically modulated by the level of aggression displayed when interacting with the provoking opponent; this means that the stronger the retaliation in highly provocative situations the more insular cortex is recruited. 


\section{CHAPTER 6}

We could confirm our specific hypothesis that activation within the insular cortex related to retaliation is left-lateralized and mainly localized in the posterior segment: Although the entire insular cortex was activated during retaliation (group analyses), it was specifically the activation level of the left posterior insular cortex that varied with the amount of aggression displayed. This indicates functional involvement, lateralization, and segregation of the insular cortex specific to retaliation.

Craig (2009) suggested, that the insular cortex is structured along a posterior-toanterior gradient representing the progressive integration of bodily feelings. He argued that acute emotions or interoceptive components might be represented in the posterior parts of the insular cortex, while the anterior parts seem to code for more abstract and highly integrated constructs. Retaliation or reactive aggression trigger intense bodily sensation and immediate action tendencies and, thus, should activate the posterior insular cortex. A similar segregation of insular cortex was demonstrated for a concept rather opposite to aggression, namely love. While passionate love, which is closely related to intense body sensation and action-oriented, involves posterior parts of the insular cortex, companionate love involves more anterior parts (Cacioppo et al., 2013; Cacioppo, Bianchi-Demicheli, Frum, Pfaus, \& Lewis, 2012).

Although these findings are consistent with Craig's (2009) view and shed light on the neural correlates of rather abstract concepts such as aggression and love, the question remains in how far insular involvement is specific to any of these functions. In fact, the insular cortex has been associated with even more functions that are seemingly contradictory to what is reported here such as self-awareness, motor inhibition, processing of positive emotions, processing of negative emotions, and others (for review see Craig, 2009). In the context of cognitive control, the insular cortex has furthermore been described as a region modulating with stimulus saliency or urgency (Menon \& Uddin, 2010; Nelson et al., 2010; Sterzer \& Kleinschmidt, 2010). However, instead of focusing on rather isolated single processes, as often done in functional brain research, social and emotional contexts should be taken into account when explaining the functional roles of brain regions. For instance, Reynolds and Berridge (2008) demonstrated that varying emotional environments retunes the function of neural populations. They showed that neurons in the nucleus accumbens of rats encode alternately for fear or pleasure depending on the environment the animal is exposed to (home-like, versus low stress, versus high stress). This is a revolutionary finding, potentially suggesting that neural components alter their functional involvement according to the social situation in which they are recruited. The posterior insular cortex might not be exclusively involved in aggression, passionate love, or other concepts. Rather, it might be highly relevant in different circumstances of intense emotions which require consequent behavioural responses. Most probably, it thereby adapts its function to whatever requirements have to be met. Further research, involving methodology reaching beyond hemodynamic neuroimaging techniques and taking into account varying emotional environments, is needed. Moreover, it should be noted that 
the insular cortex is highly interconnected with various brain regions and it will be of interest to investigate its specific functional interactions with those regions during different emotional and social contexts.

Further activation during the decision phase. The insular cortex was not the only brain region activated during the decision phase; activation in superior temporal gyrus and primary motor cortex was also detected.

Activation in the right superior temporal gyrus was detected, when reacting mildly to the non-provoking opponent. This brain region has been associated with processes linked to social cognition (Pelphrey, Morris, \& McCarthy, 2004; Pelphrey, Viola, \& McCarthy, 2004; Zilbovicius et al., 2006). Such processes are expected to be active when the participant is confronted with the non-provoking opponent; compared to his mean companion, he is friendly, nice, and from the participants' point of view more understandable and accessible, thus an object for self-identification.

Additionally, primary motor cortex activity was detected during the decision phase. Low punishment levels $(1,2,3,4)$ had to be selected by the left hand (leading to activation in the right primary motor cortex), whereas high levels of punishment $(5,6,7,8)$ had to be selected by the right hand (leading to activation in left primary motor cortex). This mechanism is mirrored in our results: In the group analyses, the left motor cortex is activated during aggressive reactions towards the provoking opponent, while the right motor cortex is activated during non-aggressive reactions to the nonprovoking opponent. Accordingly, activity in the right motor cortex modulated with the intensity of the chosen punishment independent of the provocation condition; the higher the chosen punishment, the more involvement of the left motor cortex was observed.

Winning and losing. Winning was associated with vast neural activity in bilateral superior and middle frontal regions, the inferior parietal lobes, the left precuneus, and bilateral striatum. This is in line with previous results (Krämer et al., 2007). Striatal activation on one hand and the involvement of prefrontal areas on the other hand, strongly suggest the recruitment of the reward circuit in the brain (Haber \& Knutson, 2010). Winning during the TAP is rewarding in two ways: Outperforming the opponent in the given task (reaction time competition) might be rewarding in itself. Furthermore, winning means avoiding a punishment and the same time administering punishment to the opponent, which might also be a pleasurable experience.

In contrast, losing was associated with bilateral activation in the superior temporal gyri and the parahippocampal gyri. The former might simply reflect the anticipated auditory stimulus, which is to come every time a participant loses (and never, when the participant wins). Previously, the parahippocampal gyri were shown to be involved in scene recognition and the detection of paralinguistic speech profiles often related to the social component of the situation (e.g. sarcasm; Rankin et al., 2009). This might reflect the paralinguistic and socially driven interpretation of the communication during the outcome phase; although the information presented during this phase is objec- 
tive and seemingly neutral ("You won/ you lost" and "Your opponent chose x"), it contains social and paralinguistic cues related to the perception of the social opponent and the interpretation of his behaviour.

The only opponent-specific activation regarding the outcome phase was detected when loosing against the non-provoking opponent instead of the provoking opponent. When no highly aversive stimulus had to be expected, the parietal lobes and the left middle temporal gyrus were significantly activated. The participant might feel relief, when losing against the non-provoking opponent instead of the provoking opponent, as the noise feedback which is about to come is much less aversive. However, an association between the detected brain regions and the described processes has not been investigated yet.

Generally, the neural correlates of winning and losing were not linked to the individual levels of displayed aggression; no parametric modulations in any brain regions could be detected during the outcome phase.

\section{CONCLUSION}

This study demonstrates that the left posterior insular cortex is the core brain region involved in retaliation; this was specifically demonstrated for provocative versus nonprovocative social interactions. We employed random effects group analyses and examined parametric modulations of brain activity during a controlled behavioural aggression paradigm. The left-lateralization of insular activity during retaliation is in line with evidence from electro-physiological studies, suggesting left-lateralized frontocortical dominance during anger processing and aggressive acts. Furthermore, our results support the theory that particularly the posterior segment of insular cortex is involved in the processing of emotions triggering intense bodily sensations and immediate action tendencies. 
Anderson, C. A., Lindsay, J. J., \& Bushman, B. J. (1999). Research in the Psychological Laboratory: Truth or Triviality? Current Directions in Psychological Science, 8(1), 3-9.

Bernstein, S., Richardson, D., \& Hammock, G. (1987). Convergent and discriminant validity of the Taylor and Buss measures of physical aggression. Aggressive Behavior, 13(1), 15-24.

Beyer, F., Münte, T. F., Erdmann, C., \& Krämer, U. M. (2014). Emotional reactivity to threat modulates activity in mentalizing network during aggression. Social Cognitive and Affective Neuroscience, 9(10), 155260.

Beyer, F., Münte, T. F., Göttlich, M., \& Krämer, U. M. (2014). Orbitofrontal Cortex Reactivity to Angry Facial Expression in a Social Interaction Correlates with Aggressive Behavior. Cerebral Cortex, in print, doi:10.1093/cercor/bhu101.

Brunnlieb, C., Münte, T. F., Krämer, U., Tempelmann, C., \& Heldmann, M. (2013). Vasopressin modulates neural responses during human reactive aggression. Social Neuroscience, 8(2), 148-64.

Cacioppo, S., Bianchi-Demicheli, F., Frum, C., Pfaus, J. G., \& Lewis, J. W. (2012). The common neural bases between sexual desire and love: a multilevel kernel density fMRI analysis. The Journal of Sexual Medicine, 9(4), 1048-54.

Cacioppo, S., Couto, B., Bolmont, M., Sedeno, L., Frum, C., Lewis, J. W., ... Cacioppo, J. T. (2013). Selective decision-making deficit in love following damage to the anterior insula. Current Trends in Neurology, 7, $1-5$.

Craig, A. D. B. (2009). How do you feel - now? The anterior insula and human awareness. Nature Reviews Neuroscience, 10(1), 59-70.

Dambacher, F., Sack, A. T., Lobbestael, J., Arntz, A., Brugman, S., \& Schuhmann, T. (2014). Out of control Evidence for anterior insula involvement in motor impulsivity and reactive aggression. Social Cognitive and Affective Neuroscience, in print, doi:10.1093/scan/nsu077

Deichmann, R., Gottfried, J. A., Hutton, C., \& Turner, R. (2003). Optimized EPI for fMRI studies of the orbitofrontal cortex. Neurolmage, 19(2), 430-41.

Forman, S. D., Cohen, J. D., Fitzgerald, M., Eddy, W. F., Mintun, M. A., \& Noll, D. C. (1995). Improved assessment of significant activation in functional magnetic resonance imaging (fMRI): use of a cluster-size threshold. Magnetic Resonance in Medicine, 33(5), 636-47.

Giancola, P. R., \& Zeichner, A. (1995). Construct validity of a competitive reaction-time aggression paradigm. Aggressive Behavior, 21(3), 199-204.

Haber, S. N., \& Knutson, B. (2010). The reward circuit: linking primate anatomy and human imaging. Neuropsychopharmacology, 35(1), 4-26.

Harmon-Jones, E. (2004). Contributions from research on anger and cognitive dissonance to understanding the motivational functions of asymmetrical frontal brain activity. Biological Psychology, 67(1-2), 51-76.

Krämer, U. M., Jansma, H., Tempelmann, C., \& Münte, T. F. (2007). Tit-for-tat: the neural basis of reactive aggression. Neurolmage, 38(1), 203-11.

Lancaster, J. L., Rainey, L. H., Summerlin, J. L., Freitas, C. S., Fox, P. T., Evans, A. C., ... Mazziotta, J. C. (1997). Automated labeling of the human brain: a preliminary report on the development and evaluation of a forward-transform method. Human Brain Mapping, 5(4), 238-42.

Lancaster, J. L., Woldorff, M. G., Parsons, L. M., Liotti, M., Freitas, C. S., Rainey, L., ... Fox, P. T. (2000). Automated Talairach atlas labels for functional brain mapping. Human Brain Mapping, 10(3), 120-31.

Lotze, M., Veit, R., Anders, S., \& Birbaumer, N. (2007). Evidence for a different role of the ventral and dorsal medial prefrontal cortex for social reactive aggression: An interactive fMRI study. Neurolmage, 34(1), 470-8.

Menon, V., \& Uddin, L. Q. (2010). Saliency, switching, attention and control: a network model of insula function. Brain Structure \& Function, 214(5-6), 655-67.

Nelson, S. M., Dosenbach, N. U. F., Cohen, A. L., Wheeler, M. E., Schlaggar, B. L., \& Petersen, S. E. (2010). Role of the anterior insula in task-level control and focal attention. Brain Structure \& Function, 214(56), 669-80. 


\section{CHAPTER 6}

Pelphrey, K. A., Morris, J. P., \& McCarthy, G. (2004). Grasping the intentions of others: the perceived intentionality of an action influences activity in the superior temporal sulcus during social perception. Journal of Cognitive Neuroscience, 16(10), 1706-16.

Pelphrey, K. A., Viola, R. J., \& McCarthy, G. (2004). When strangers pass: processing of mutual and averted social gaze in the superior temporal sulcus. Psychological Science, 15(9), 598-603.

Rankin, K. P., Salazar, A., Gorno-Tempini, M. L., Sollberger, M., Wilson, S. M., Pavlic, D., ... Miller, B. L. (2009). Detecting sarcasm from paralinguistic cues: anatomic and cognitive correlates in neurodegenerative disease. Neurolmage, 47(4), 2005-15.

Reynolds, S. M., \& Berridge, K. C. (2008). Emotional environments retune the valence of appetitive versus fearful functions in nucleus accumbens. Nature Neuroscience, 11(4), 423-5.

Sanfey, A. G., Rilling, J. K., Aronson, J. a, Nystrom, L. E., \& Cohen, J. D. (2003). The neural basis of economic decision-making in the Ultimatum Game. Science, 300(5626), 1755-8.

Sterzer, P., \& Kleinschmidt, A. (2010). Anterior insula activations in perceptual paradigms: often observed but barely understood. Brain Structure \& Function, 214(5-6), 611-22.

Talairach, J., \& Tournoux, P. (1988). Co-planar stereotaxic atlas of the human brain: an approach to medical cerebral imaging.

Taylor, S. P. (1967). Aggressive behavior and physiological arousal as a function of provocation and the tendency to inhibit aggression. Journal of Personality, 35(2), 297-310.

Van Honk, J., \& Schutter, D. J. L. G. (2006). From affective valence to motivational direction: the frontal asymmetry of emotion revised. Psychological Science, 17(11), 963-5.

Zilbovicius, M., Meresse, I., Chabane, N., Brunelle, F., Samson, Y., \& Boddaert, N. (2006). Autism, the superior temporal sulcus and social perception. Trends in Neurosciences, 29(7), 359-66. 




\section{CHAPTER 7}

\section{Reducing proactive aggression through Non-Invasive Brain Stimulation}

"If he can only perform good or only perform evil, then he is a clockwork orangemeaning that he has the appearance of an organism lovely with colour and juice but is in fact only a clockwork toy to be wound up by God or the Devil."

- Anthony Burgess, A Clockwork Orange, 1962 
Based on: Dambacher*, F., Schuhmann*, T., Lobbestael, J., Arntz, A., Brugman, S., \&-Sack, A. T. (2015). Reducing proactive aggression through Non-Invasive Brain Stimulation. Social cognitive and affective Neuroscience, in print.

* both authors contributed equally

We thank Jaqueline Baker, Vincent Broens, Lara Gaertner, Lukas Henning, Lennard Klein, Lara Nicolaysen, and Marly Schmitz for the work they invested in this project. 


\section{ABSTRACT}

Aggressive behaviour poses a threat to human collaboration and social safety. It is of utmost importance to identify the functional mechanisms underlying aggression and to develop potential interventions capable of reducing dysfunctional aggressive behaviour already at a brain level. We here experimentally shifted fronto-cortical asymmetry to manipulate the underlying motivational emotional states in both male and female participants while assessing the behavioural effects on proactive and reactive aggression. Thirty-two healthy volunteers received either anodal transcranial direct current stimulation to increase neural activity within right dorsolateral prefrontal cortex, or sham stimulation. Aggressive behaviour was measured with the Taylor Aggression Paradigm (TAP). We revealed a general gender effect, showing that males displayed more behavioural aggression than females. After the induction of right frontohemispheric dominance, proactive aggression was reduced in males. The current study demonstrates that Non-Invasive Brain Stimulation can reduce aggression in males. This is a relevant and promising step to better understand how cortical brain states connect to impulsive actions and to examine the causal role of the prefrontal cortex in aggression. Ultimately such findings could help to examine whether the brain can be a direct target for potential supportive interventions in clinical settings dealing with overly aggressive patients and/or violent offenders. 
Aggression is behaviour that intentionally causes physical or psychological harm to another being (Anderson \& Bushman, 2002). It has a wide range of possible expressions and has been categorized into different subtypes based on distinct motivations: while reactive aggression refers to aggressive behaviour in reaction to provocation, proactive aggression refers to using aggression in an instrumental way (Poulin \& Boivin, 2000; Raine et al., 2006). Aggression poses a threat to human collaboration and social safety. Aggressive reactions can lead to severe criminal acts putting potential victims at risk, but also destroying the lives of offenders and posing enormous costs to society. It is, therefore, crucial to understand the societal, cognitive, and neurobiological mechanisms underlying aggression. This knowledge can lead to the development of interventions that can reduce overly aggressive behaviour. In this study we used NonInvasive Brain Stimulation to attempt to reduce aggression.

Research has repeatedly tackled the question of why some individuals are highly aggressive, whereas others are not. The General Aggression Model (Anderson \& Bushman, 2002) states that aggressive behaviour results from an interplay between personal and situational variables and is mediated by cognitive, affective, and arousalrelated processes within an individual. One of the cognitive mechanisms playing a role in aggressive behaviour is the processing of social cues such as, for instance, social situation, social counterpart, or social content of communication (Crick \& Dodge, 1994). Social cue processing is biased in aggressive individuals (Crick \& Dodge, 1996). In this context, pro- and reactive aggression could consistently be dissociated as two distinct types of aggression. For instance, behavioural research showed that a hostile interpretation style and an attentional bias towards angry faces was related to reactive aggression, whereas a stronger self-aggression association was shown to be related to proactive aggression (Brugman et al., 2014; Lobbestael, Cima, \& Arntz, 2013).

More recently, neuroscientific research has identified potential neural substrates underlying aggression as one form of (anti)social behaviour. Brain researcher studies have investigated behavioural aggression in healthy adults (Krämer, Jansma, Tempelmann, \& Münte, 2007; Krämer, Riba, Richter, \& Münte, 2011; Lotze, Veit, Anders, \& Birbaumer, 2007), adolescents (White, Brislin, Meffert, Sinclair, \& Blair, 2013), and psychopaths (Veit et al., 2010): Neural networks associated with aggression included various regions within prefrontal cortex, the insular cortex, the cingulate cortex, striatal areas, and the amygdala (Krämer et al., 2007, 2011; Lotze et al., 2007; Veit et al., 2010; White et al., 2013). Subsequently, attempts have been made to relate these brain networks to other executive networks. For instance, overlapping areas activated during aggressive behaviour and failed motor inhibition could be located in prefrontal cortex (more specifically anterior insula) and thalamus (Dambacher et al., 2014). Specifically prefrontal cortex has repeatedly been associated with cognitive control (Koechlin, Ody, \& Kouneiher, 2003; Miller, 2000). Dual-path theories emphasize the role of prefrontal cortex as a mediator for subcortical communication (Ledoux \& Phelps, 2003). An example of the prefrontal cortex as a mediator can be found in the 
communication between thalamus and amygdala. They can communicate via a direct pathway and this communication leads to rapid responses following emotional stimuli, but the responses are very unspecific. When signals from one subcortical region to the other are, however, directed through the prefrontal cortex, responses become more elaborate, though slower. Within the prefrontal cortex inter-hemispheric balance determines the affective motivational state: Motivational direction is the basic psychological domain related to hemispheric asymmetry (van Honk \& Schutter, 2006). Whereas avoidance or withdrawal motivation is mainly associated with right frontocortical brain activity, approach motivation is related to activity in the left prefrontal cortex (Davidson, 1992; Harmon-Jones \& Allen, 1998; Harmon-Jones \& Sigelman, 2001; Harmon-Jones, 2004). In the context of aggression, anger-related (thus approachrelated) brain states have also been allocated to the left prefrontal cortex (Carver \& Harmon-Jones, 2009). Based on this concept of fronto-cortical asymmetry, it has been demonstrated that through contractions of the right hand, greater left-compared to right-hemispheric frontal activity (measured by electro encephalogram) was induced, which led to increased aggression after provocation (Peterson, Shackman, \& HarmonJones, 2008).

The experimental induction of either left or right fronto-cortical dominance seems promising to understand how cortical balance theories can translate to behaviour. Non-Invasive Brain Stimulation methods such as Transcranial Magnetic Brain Stimulation (TMS) or transcranial Direct Current Stimulation (tDCS) are mechanistic or causal techniques that are able to further clarify the role of the prefrontal cortex in mediating aggressive behaviour. While TMS can enhance or disturb brain activity in a specific region by means of electromagnetic induction, tDCS induces low electric currents into brain tissue to either de- or increase the excitability of the stimulated areas. Until hitherto surprisingly few studies investigated the role of prefrontal cortex in aggression using Non-Invasive Brain Stimulation techniques: The induction of relative left frontocortical activation by means of tDCS was shown to increase aggressive behaviour in a reaction time game (Hortensius, Schutter, \& Harmon-Jones, 2012). Similar mechanisms were demonstrated in the attentional domain: Increased left-to-right and simultaneously reduced right-to-left transcallosal inhibition (measured via motor evoked potentials induced by TMS) was associated with a stronger attentional bias for angry faces (Hofman \& Schutter, 2009). Furthermore, the disruption of right prefrontal cortex by means of repetitive TMS (and, thus, induction of relative left frontal brain activity) shifted selective attention towards angry faces (d'Alfonso, van Honk, Hermans, Postma, \& de Haan, 2000).

The described results from behavioural aggression research indicate that different aspects of aggressive behaviour - i.e. pro- versus reactive aggression - are dissociable. This suggests that also the neural mechanisms underlying these different forms of aggression might be different. The described neuroscientific findings indicate that shifting fronto-cortical balance can affect cognitive mechanisms underlying aggression 


\section{CHAPTER 7}

(d'Alfonso et al., 2000; Hofman \& Schutter, 2009) and lead to more aggressive behaviour (Hortensius et al., 2012). Particularly, right-hemispheric fronto-cortical dominance was found to be related to avoidance- or withdrawal-related behaviour. Increasing activity in this area should decrease aggressive behaviour by increasing avoidance, as compared to approach, motivation. So far it has not been investigated if the induction of right fronto-cortical dominance can experimentally reduce different aspects of aggression in a controlled behavioural aggression paradigm. Reducing aggression under controlled experimental conditions in healthy volunteers is necessary to causally clarify the role of the right prefrontal cortex in mediating aggressive behaviour. To directly provide this missing piece of evidence, we investigated whether shifting fronto-cortical balance by means of tDCS affects pro- and / or reactive aggression in healthy participants. We expected that the induction of right hemispheric fronto-cortical dominance by applying tDCS over right dorsolateral prefrontal cortex should enhance avoidance motivation and, thereby, cause a significant reduction in aggressive behaviour as compared to sham tDCS. Furthermore we expected that this reduction would differentially affect pro- as compared to reactive aggression.

\section{MATERIALS AND METHODS}

Participants. Fourty-three healthy university students ( $\mathrm{N}=20$ male; mean age in years=22.14; $S D=2.00$ ) took part in this study. All had no history of neurological or psychiatric disorders and gave their written informed consent before participating.

Paradigms and tools. Taylor Aggression Paradigm (TAP). To measure aggressive behaviour, a standard controlled behavioural aggression paradigm was employed (Taylor, 1967): Participants were made to believe that they played a competitive reaction time game against another participant sitting in the room next door. The amount of win and lose trials were preprogrammed and the players were made to believe that the winner of a trial could administer a loud noise to the looser as "feedback". Before each trial, the participants were asked to choose the duration and volume of this noise blast on a 10-point scale (volume: 0 to $100 \mathrm{~dB}$; duration: 0 to 5 seconds). 30 trials were played. The first provocation (first noise feedback not being zero) was given in the 7th trial. Three aggression scores could be calculated: A proactive aggression score was calculated by summating intensity- and duration-scores for the unprovoked (first seven) trials. A reactive aggression score was calculated by summating intensity- and duration-scores for the provoked (last 23) trials. A total aggression score was calculated by summating and averaging intensity- and duration-scores across all trials. The TAP has previously demonstrated high validity (Anderson, Lindsay, \& Bushman, 1999; Bernstein, Richardson, \& Hammock, 1987; Giancola \& Parrott, 2008; Giancola \& Zeichner, 1995). 
Reactive-Proactive-Aggression Questionnaire (RPQ). The Reactive Proactive Aggression Questionnaire (Raine et al., 2006) was used to measure self-reported trait aggression. Twelve items measured proactive aggression (e.g. 'Used physical force to get others to do what you want'), while eleven items measured reactive aggression (e.g. 'Reacted angrily when provoked by others'). By taking all 23 items into account an overall total aggression score could be calculated. High internal reliability has been shown for all scales ( $\alpha=0.81$ for reactive aggression, $\alpha=0.84$ for proactive aggression, $\alpha=0.90$ for total aggression; Raine et al., 2006).

Experimental design. To assure that behaviour in the experiment was unaffected by social desirability, participants were told that they took part in a study investigating the effects of human feedback on reaction time performance. In every experimental session two participants of the same gender took part simultaneously.

The tDCS setup was mounted on the participants' heads and they received instructions about the task. After the brain stimulation was initialized, participants performed the TAP. Immediately after completion of the experiment, participants had to answer some general questions about how they perceived the task in order to make sure that they were fully deceived by the experimental setup. Twenty-four hours after completing the experiment, participants had to fill in the RPQ.

After all the measurements had been finalized the experiments' real purpose and motivation was disclosed to the participants. The study was approved by the local ethical committee and conformed to the Declaration of Helsinki.

Non-Invasive Brain Stimulation. Three participants had to be excluded as they were suspicious about the real purpose of the investigation. Another 8 participants had to be excluded since their medical conditions at the day of the experiment did not allow for the application of brain stimulation. Thirty-two participants could be included in the analysis and were randomly assigned to one of two tDCS conditions: stimulation over right dorsolateral prefrontal cortex $\mathrm{N}=16(\mathrm{~N}=7$ male), sham stimulation $\mathrm{N}=16(\mathrm{~N}=6$ male). As the TAP relies on participant naivety it is not suited be repeated in a withinsubject-design. Even though the TAP is the best possible measure of our dependent variable, we sacrificed a possible within-subject-design.

To induce right hemispheric fronto-cortical dominance - and thus enhance avoidance motivation - the anode was positioned over the right dorsolateral prefrontal cortex (F4), while the cathode was positioned above the left eyebrow (figure 1). A DCstimulator with $5 \times 7 \mathrm{~cm}$ standard electrodes (neuroConn, Ilmenau, Germany) was used. We induced 2.0 milliampere direct current for a duration of 750 seconds (ramping phases 20 seconds each). To apply sham tDCS, the same procedure was followed as explained above, but the stimulation was switched off immediately after the ramping phases. This mimicked the skin sensation accompanying real tDCS application and deceived participants about which condition they were assigned to. Unlike sham TMS, sham tDCS feels identical to real tDCS can thus be regarded as a highly effective sham condition. 


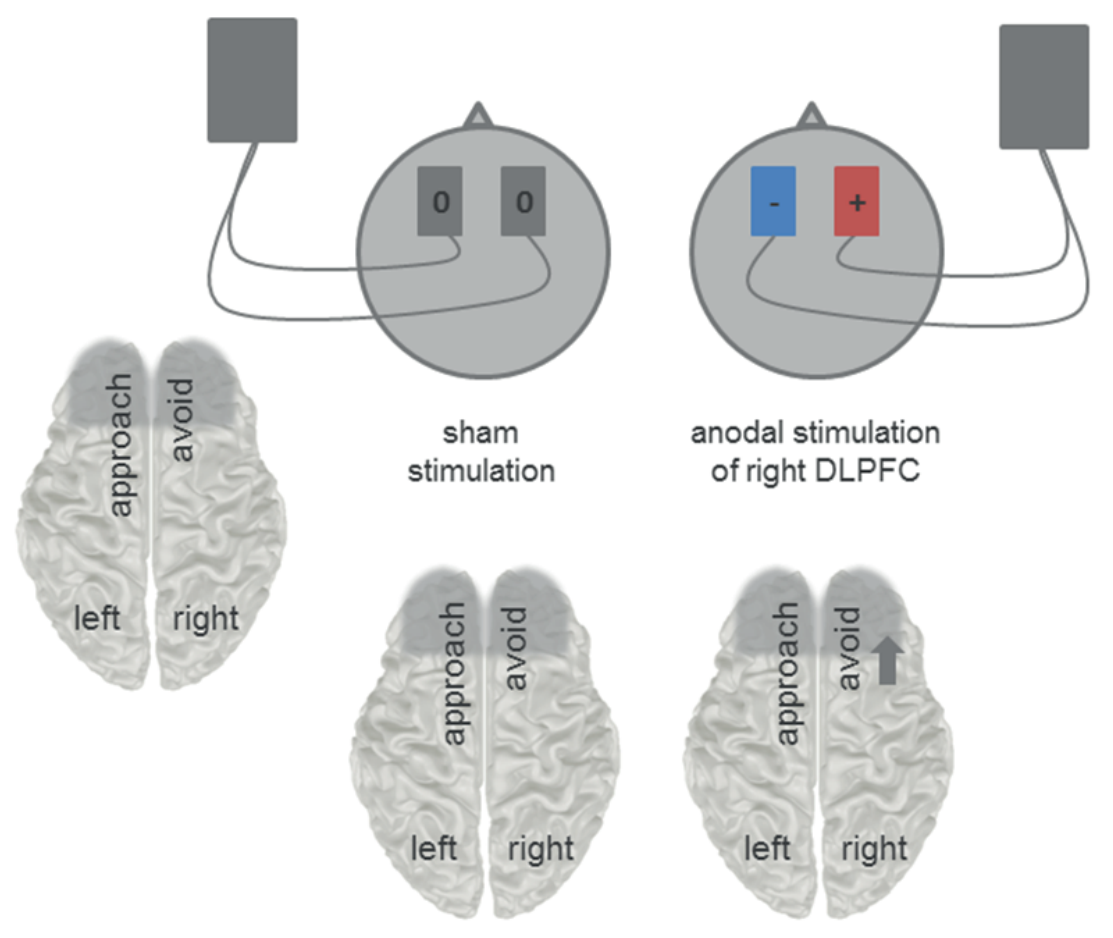

Fig 1. TDCS setup. DLPFC (dorsolateral prefrontal cortex).

Statistical analysis. Inferential statistics were conducted by computing multivariate analyses of variance (MANOVA) with the $2 \times 2$ factors gender (male, female) and stimulation condition (induction of right hemispheric dominance, sham stimulation). Total aggression, reactive aggression, and proactive aggression were included as dependent variables. This was done for both the RPQ and the TAP separately. When a significant interaction effect was found, the sample was split and post-hoc tests were conducted via independent sample t-tests. Bivariate Pearson product-moment correlations coefficients were computed to estimate the relation between TAP and RPQ.

\section{RESULTS}

Mean values and standard deviations are summarized in table 1 . Results are depicted in figure 2 and figure 3.

Gender. A multivariate analysis of variance showed that males behaved more aggressively (total aggression and reactive aggression) than females, regardless of stimulation type (TAP; total aggression $F=5.33 / d f=1,62 / p=.029$, reactive aggression $F=4.31$ / $\mathrm{df}=1,62$ / $\mathrm{p}=.047$; proactive aggression $\mathrm{F}=3.94 / \mathrm{df}=1,62$ / $\mathrm{p}=.057$; figure $2 \mathrm{~A}$ ). Males considered themselves more proactively aggressive (RPQ; total aggression $F=7.49$ / 
$\mathrm{df}=1,62 / \mathrm{p}=.011$, reactive aggression $\mathrm{F}=2.84 / \mathrm{df}=1,62 / \mathrm{p}=.103 ;$ proactive aggression $F=13.42 / d f=1,62 / p=.001$; figure 2B).

Tab1. Mean and standard deviation (SD) per gender and stimulation condition. TAP (Taylor Aggression Paradigm; descriptive statistics based on mean), RPQ (reactive-proactive-aggression questionnaire; descriptive statistics based on sum scores).

\begin{tabular}{|c|c|c|c|c|c|c|c|c|c|c|c|c|}
\hline & \multirow{2}{*}{\multicolumn{2}{|c|}{ tDCS }} & \multirow{2}{*}{\multicolumn{2}{|c|}{ sham }} & \multicolumn{4}{|l|}{ male } & \multicolumn{4}{|c|}{ female } \\
\hline & & & & & \multicolumn{2}{|l|}{ tDCS } & \multicolumn{2}{|l|}{ sham } & \multicolumn{2}{|l|}{ tDCS } & \multicolumn{2}{|c|}{ sham } \\
\hline & $M$ & SD & $M$ & SD & $M$ & SD & $M$ & SD & $M$ & SD & $M$ & SD \\
\hline TAP & 3.84 & 1.16 & 4.00 & 1.33 & 4.16 & .90 & 4.87 & .86 & 3.60 & 1.33 & 3.48 & 1.32 \\
\hline total & 4.07 & 1.25 & 4.13 & 1.48 & 4.52 & .96 & 4.86 & 1.09 & 3.73 & 1.39 & 3.69 & 1.55 \\
\hline reactive & 2.93 & 1.25 & 3.49 & 1.72 & 2.74 & 1.26 & 4.89 & 1.50 & 3.08 & 1.29 & 2.69 & 1.27 \\
\hline \multicolumn{13}{|l|}{ proactive } \\
\hline $\mathrm{RPQ}$ & 8.44 & 3.44 & 9.31 & 6.16 & 10.00 & 5.98 & 13.17 & 3.15 & 7.22 & 2.95 & 7.00 & 5.25 \\
\hline total & 7.00 & 2.34 & 7.00 & 4.03 & 7.71 & 3.67 & 8.67 & 3.67 & 6.44 & 2.56 & 6.00 & 4.08 \\
\hline proactive & 1.44 & 1.67 & 2.31 & 2.75 & 2.29 & 3.15 & 4.50 & 5.98 & .78 & .83 & 1.00 & 1.41 \\
\hline reactive & 3.84 & 1.16 & 4.00 & 1.33 & 4.16 & .90 & 4.87 & .86 & 3.60 & 1.33 & 3.48 & 1.32 \\
\hline
\end{tabular}

Correlations between behavioural and self-report measures. Proactive behavioural aggression (TAP) correlated positively with self-reported proactive aggression (RPQ) in males, but not in females. For the other types of aggression there was no relation between behavioural and self-report measures (over all sample: total aggression $r=.27 / p=.132$, reactive aggression $r=.20 / p=.266$, proactive aggression $r=.50 / p=.003$; male: total aggression $r=-.13 / p=.678$, reactive aggression $r=-.400 / p=.175$, proactive aggression $r=.62 / p=.024$; female: total aggression $r=.27 / p=.268$, reactive aggression $r=.29 / p=.225$, proactive aggression $r=.059 / p=.809$ ). 

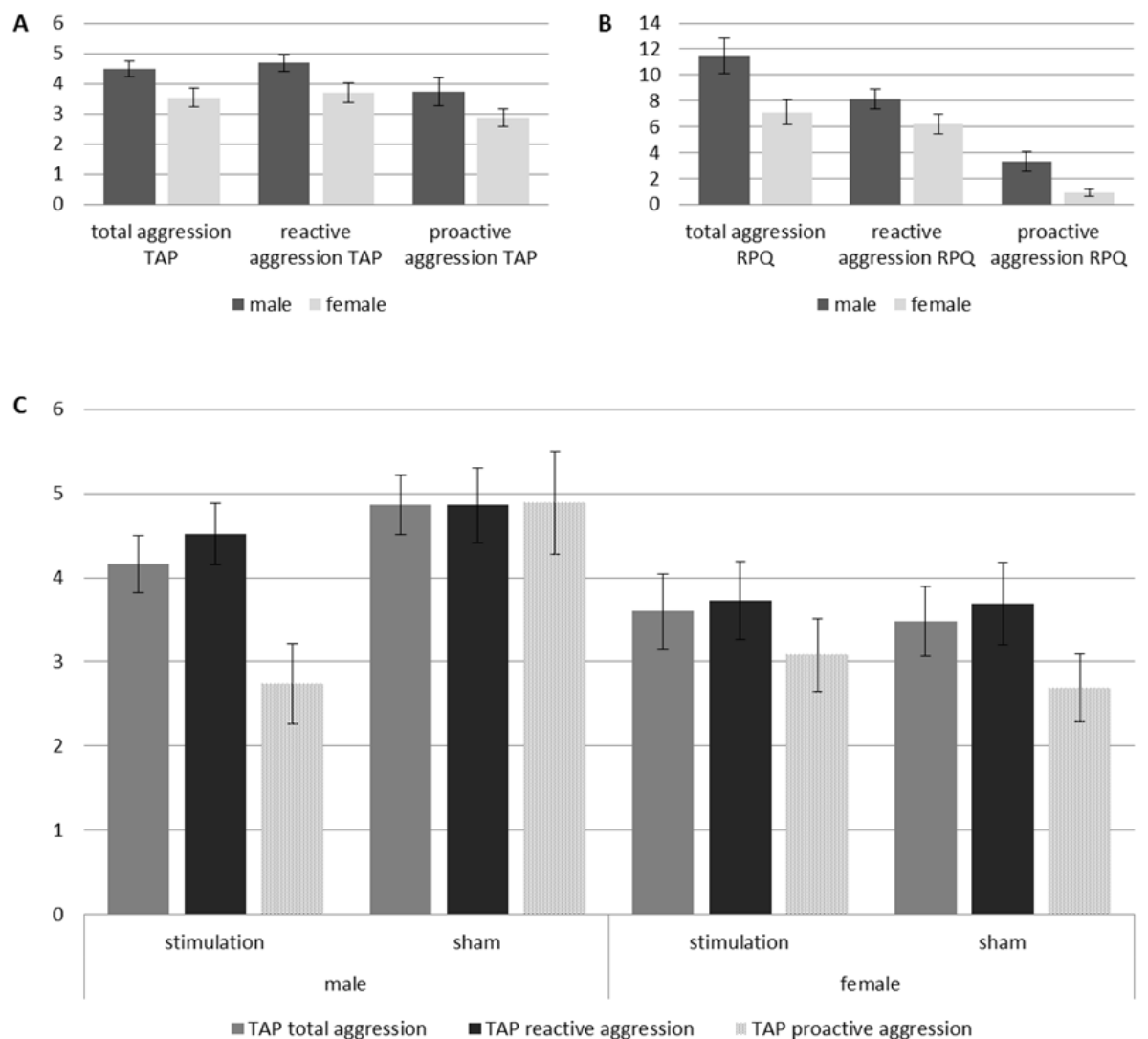

Fig 2. Aggression scores per gender and stimulation condition. TAP (Taylor Aggression Paradigm; descriptive statistics based on mean), RPQ (reactive-proactive-aggression questionnaire; descriptive statistics based on sum scores).

TDCS effects. For total and reactive aggression in the TAP, a multivariate analysis of variance revealed that there was no main effect of stimulation condition (total aggression $F=.485 / d f=1,62 / p=.492$; reactive aggression $F=.103 / d f=1,62 / p=.750$ ). No interaction effects between stimulation condition and gender were found (total aggression $F=.953 / d f=1,62 / p=.337$; reactive aggression $F=.167 / d f=1,62 / p=.686)$. In contrast, for proactive aggression, there was a significant interaction effect between gender and stimulation condition $(\mathrm{F}=7.35 / \mathrm{df}=1,62 / \mathrm{p}=.011)$, with post hoc contrast analyses revealing that this interaction was driven by the induction of right hemispheric dominance significantly reducing proactive aggression in males (MEAN=2.74\&MEAN=4.89/ $d f=11 / p=.018 /$ Cohen's $d=1.55$; figure $2 C$ \& figure 3 ), but not in females (MEAN=3.08\& MEAN=2.66/df $=17 / p=.480 /$ Cohen's $d=0.33$; figure $2 C$ ). Reactive aggression was not altered by brain stimulation in either males $(\mathrm{MEAN}=4.52 \& M E A N=4.86 / \mathrm{df}=11 / \mathrm{p}=.554$ / 
Cohen's $d=0.34$; figure 2C) nor females $(M E A N=3.73 \& M E A N=3.69 / d f=17 / p=.952 /$ Cohen's $d=0.03$; figure $2 \mathrm{C}$ ).

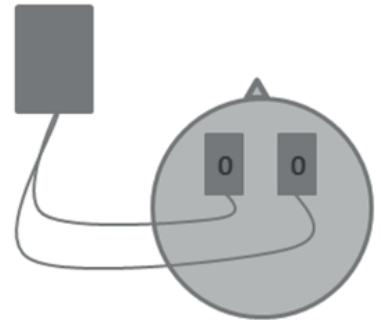

sham stimulation

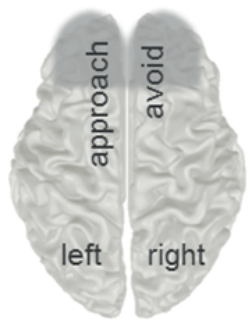

proactive aggression reactive aggression

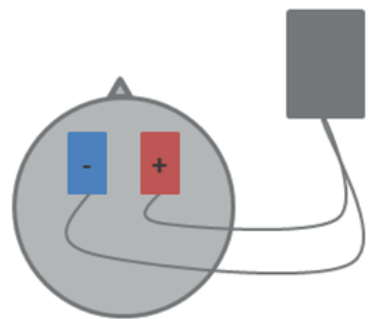

anodal stimulation of right DLPFC

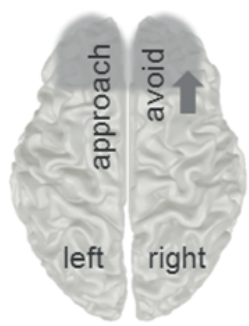

proactive aggression

reactive aggression

Fig 3. Results. DLPFC (dorsolateral prefrontal cortex).

\section{DISCUSSION}

This study revealed that anodal compared to sham tDCS applied to the right dorsolateral prefrontal cortex reduced proactive aggression in men.

Gender differences. Exploring gender differences in our sample, we demonstrated that men reported more aggressive tendencies than women did. They also behaved more aggressively compared to women. . A vast body of literature is in line with this finding. It has repeatedly been suggested that males display more physical aggression than females, who in turn tend to revert to more indirect forms of aggression (Archer, 2004; Bjorkqvist, 1994; Eagly \& Steffen, 1986; Lagerspetz et al., 1988). Several biological factors such as testosterone levels contribute to this phenomenon (Book, Starzyk, \& Quinsey, 2001; Mehta \& Beer, 2010). The Taylor Aggression Paradigm, in which the actual aggressive act is to assign to the opponent a noise feedback evoking a rather 
unpleasant and almost painful auditory experience, can be understood as measure of physical aggression. It is therefore to be expected that - due to its characteristics - the TAP is well suited to generate aggression in males.

Relation between behavioural and self-reported aggression. There was no relationship between total and reactive behavioural and self-reported aggression scores. It is a long- and well-known problem in aggression research that behavioural measures of social constructs do not necessarily overlap with measures on a self-reported level (Scheier et al., 1978). Especially in this domain, effects of social desirability are obstacles that measurement tools have to overcome (Vigil-Colet et al., 2012). Our selfreport data was probably likewise affected as we measured exclusively university students, a sample for which it might be very difficult to admit aggressive tendencies.

We found a positive relation between behavioural and self-reported aggression in the proactive domain for the overall sample and for males. This might hint towards the fact that conceptually, the proactive aspect of the TAP overlaps more precisely with the proactive sub-scale of the RPQ than the reactive aspect of the TAP with the reactive sub-scale of the RPQ. Biases regarding self-reported aggression might be more relevant for reactive than for proactive aggression. In our societies it is emphasized that everyone should react rationally to provocation. Proactive aggression might be less frequent and more exceptional and, thus, less prone to social biases. The data collected in this study could give a hint in this direction. However, more empirical evidence needs to be collected in larger samples to substantiate this claim.

Effects of brain stimulation. In line with our hypothesis, we found that the induction of right hemispheric neural activation dominance reduced aggressive behaviour compared with sham brain stimulation, although the effect was only significant in males.

Dissociation of pro- versus re-active aggression. Proactive aggression refers to the instrumental use of aggression to obtain a reward or a prey (Anderson \& Bushman, 2002). Therefore, the motivation to approach seems central. The experimental manipulation in this study was meant to enhance activity in the right dorsolateral prefrontal cortex. This area is said to be responsible for emotional and cognitive processes generating avoidance motivation (Carver \& Harmon-Jones, 2009; Harmon-Jones, 2004). The assumption that the applied brain stimulation protocol enhanced avoidance and thus lowered approach motivation fits with our finding that it reduced proactive aggression.

The current findings can also be explained in the light of social informationprocessing theories. It has been shown that re- and proactive aggression revert to biases in different stages of social information processing. Thereby, reactive aggression seems to result from deviations in rather early stages, such as an increased attentional bias for angry faces or a hostile interpretation bias (Anderson \& Bushman, 2002; Lobbestael et al., 2013; Brugman et al., in press). For proactive aggression, the later stages seem more impaired and lead to a more positive evaluation of aggressive action options (Walters, 2007). A proactive attitude likely also steers coping processes, mean- 
ing that proactively aggressive individuals have the tendency to approach their goals using aggression. The evaluation of the option to act proactively aggressive is more closely related to approach motivation than to attention and interpretation biases. It seems likely that an alteration of such motivational states (on a neural level) influences proactive rather than reactive aggression.

With this study we demonstrated that it is possible to specifically manipulate proactive aggression. Usually, this form of aggression is more difficult to deal with in clinical contexts; proactive aggression is potentially very dangerous as it is planned behaviour and not emotionally driven. It is often prevalent in patients with psychopathic traits. So far, neuroscience and especially neuroimaging research mostly neglected the differentiation between pro- and reactive aggression. In the light of the current results it seems promising to consider the difference in further neuroscientific research on aggression. This could lead to more elaborate theories on which specific neural mechanisms underlie pro- compared to reactive aggression and how these mechanisms can be manipulated in order to ultimately change behaviour.

Limitations and outlook. This study demonstrates that tDCS can reduce aggressive behaviour. Our findings still have to be considered in light of the limitations that the current experimental setting was accompanied by. Our sample $(\mathrm{N}=32)$ was restricted to university students. The field would profit from investigating larger samples and more heterogeneous populations. The lack of a stimulation effect in females might be caused by a floor effect considering that female students in our restricted sample displayed low aggression levels. Applying tDCS in the context of aggression to larger and, further, more variable female sample might lead to a clearer picture on whether aggressive behaviour can or cannot be reduced in women compared to men. Furthermore, research should also zoom in on larger male samples enabling the inclusion of more control-variables (such as e.g. perception of the opponent, perception of feedback, influence of brain stimulation side effects) and different brain stimulation conditions.

In order to further examine the specificity of the present effects, other stimulation parameters, such as bilateral stimulation setups and frequency-dependent protocols, might be of use in further investigating the effects of cortical asymmetry on aggression. Future experiments should consider including different stimulation sides within the prefrontal cortex (based on imaging literature) in order to investigate, if lateralization effects are bound to the right dorsolateral prefrontal cortex.

We demonstrated effects of brain stimulation on aggression measured by the Taylor paradigm. The question of whether the findings are generalizable and specific to aggression remains to be answered. It is especially interesting to assess to what degree the very same mechanism plays a role in both aggressive and prosocial approach. 


\section{CONCLUSION}

The current study demonstrates that Non-Invasive Brain Stimulation can significantly reduce aggression, and dissociate between pro- and reactive aggression. This is a promising step in order to better understand how cortical brain states connect to aggressive behaviour. It enables the examination of how interventions in clinical settings dealing with aggression can be improved. 
Anderson, C. A., \& Bushman, B. J. (2002). Human aggression, Annual Review of Psychology, 53, 27-51.

Anderson, C. A., Lindsay, J. J., \& Bushman, B. J. (1999). Research in the Psychological Laboratory: Truth or Triviality? Current Directions in Psychological Science, 8(1), 3-9.

Archer, J. (2004). Sex Differences in Aggression in Real-World Settings: A Meta-Analytic Review. Review of General Psychology, 8(4), 291-322.

Bernstein, S., Richardson, D., \& Hammock, G. (1987). Convergent and discriminant validity of the Taylor and Buss measures of physical aggression. Aggressive Behavior, 13(1), 15-24.

Bjorkqvist, K. (1994). Sex differences in physical, verbal, and indirect aggression: A review of recent research. Sex Roles, 30(3-4), 177-188.

Book, A. S., Starzyk, K. B., \& Quinsey, V. L. (2001). The relationship between testosterone and aggression: a meta-analysis. Aggression and Violent Behavior, 6(6), 579-599.

Brugman S., Lobbestael J., Arntz A., Cima M. J., Schuhmann T., Dambacher F., Sack, A. T. (2014). Identifying cognitive predictors of reactive and proactive Aggression. Aggressive Behavior, in print, doi: 10.1002/AB.21573.

Carver, C. S., \& Harmon-Jones, E. (2009). Anger is an approach-related affect: evidence and implications. Psychological Bulletin, 135(2), 183-204.

Crick, N. R., \& Dodge, K. A. (1994). A Review and Reformulation of Social Information-Processing Mechanisms in Children's Social Adjustment. Psychological Bulletin, 115(1), 74-101.

Crick, N. R., \& Dodge, K. A. (1996). Social Information-Processing Mechanisms in Reactive and Proactive Aggression. Child Development, 67(3), 993-1002.

d'Alfonso, A. A., van Honk, J., Hermans, E., Postma, A., \& de Haan, E. H. (2000). Laterality effects in selective attention to threat after repetitive transcranial magnetic stimulation at the prefrontal cortex in female subjects. Neuroscience Letters, 280(3), 195-8.

Dambacher, F., Sack, A. T., Lobbestael, J., Arntz, A., Brugman, S., \& Schuhmann, T. (2014). Out of control: Evidence for anterior insula involvement in motor impulsivity and reactive aggression. Social Cognitive and Affective Neuroscience, in print, doi: 10.1093/scan/nsu077.

Davidson, R. J. (1992). Anterior cerebral asymmetry and the nature of emotion. Brain and Cognition, 20(1), 125-51.

Eagly, A. H., \& Steffen, V. J. (1986). Gender and aggressive behavior: a meta-analytic review of the social psychological literature. Psychological Bulletin, 100(3), 309-30.

Giancola, P. R., \& Parrott, D. J. (2008). Further evidence for the validity of the Taylor Aggression Paradigm. Aggressive Behavior, 34(2), 214-29.

Giancola, P. R., \& Zeichner, A. (1995). Construct validity of a competitive reaction-time aggression paradigm. Aggressive Behavior, 21(3), 199-204.

Harmon-Jones, E. (2004). Contributions from research on anger and cognitive dissonance to understanding the motivational functions of asymmetrical frontal brain activity. Biological Psychology, 67(1-2), 51-76.

Harmon-Jones, E., \& Allen, J. J. (1998). Anger and frontal brain activity: EEG asymmetry consistent with approach motivation despite negative affective valence. Journal of Personality and Social Psychology, 74(5), 1310-6.

Harmon-Jones, E., \& Sigelman, J. (2001). State anger and prefrontal brain activity: evidence that insultrelated relative left-prefrontal activation is associated with experienced anger and aggression. Journal of Personality and Social Psychology, 80(5), 797-803.

Hofman, D., \& Schutter, D. J. L. G. (2009). Inside the wire: aggression and functional interhemispheric connectivity in the human brain. Psychophysiology, 46(5), 1054-8.

Hortensius, R., Schutter, D. J. L. G., \& Harmon-Jones, E. (2012). When anger leads to aggression: induction of relative left frontal cortical activity with transcranial direct current stimulation increases the angeraggression relationship. Social Cognitive and Affective Neuroscience, 7(3), 342-7.

Koechlin, E., Ody, C., \& Kouneiher, F. (2003). The architecture of cognitive control in the human prefrontal cortex. Science, 302(5648), 1181-5.

Krämer, U. M., Jansma, H., Tempelmann, C., \& Münte, T. F. (2007). Tit-for-tat: the neural basis of reactive aggression. Neurolmage, 38(1), 203-11. 


\section{CHAPTER 7}

Krämer, U. M., Riba, J., Richter, S., \& Münte, T. F. (2011). An fMRI study on the role of serotonin in reactive aggression. PloS One, 6(11), e27668.

Lagerspetz, K. M. J., Bjorkqvist, K., \& Peltonen, T. (1988). Is Indirect Aggression Typical of Females ? Gender Differences in Aggressiveness in 1- to 12-year-old children. Aggressive Behavior, 14, 403-414.

Ledoux, J. E., Phelps, E. A. Emotional networks in the brain. In M. Lewis, J. M. Haviland-Jones, Handbook of emotions (2003, 2nd ed, 157-172). New York: Guilford Press.

Lobbestael, J., Cima, M., \& Arntz, A. (2013). The relationship between adult reactive and proactive aggression, hostile interpretation bias, and antisocial personality disorder. Journal of Personality Disorders, 27(1), 53-66.

Lotze, M., Veit, R., Anders, S., \& Birbaumer, N. (2007). Evidence for a different role of the ventral and dorsal medial prefrontal cortex for social reactive aggression: An interactive fMRI study. Neurolmage, 34(1), 470-8.

Mehta, P. H., \& Beer, J. (2010). Neural mechanisms of the testosterone-aggression relation: the role of orbitofrontal cortex. Journal of Cognitive Neuroscience, 22(10), 2357-68.

Miller, E. K. (2000). The prefrontal cortex and cognitive control. Nature Reviews Neuroscience, 1(1), 59-65.

Peterson, C. K., Shackman, A. J., \& Harmon-Jones, E. (2008). The role of asymmetrical frontal cortical activity in aggression. Psychophysiology, 45(1), 86-92.

Poulin, F., \& Boivin, M. (2000). Reactive and proactive aggression: Evidence of a two-factor model. Psychological Assessment, 12(2), 115-122.

Raine, A., Dodge, K., Loeber, R., Gatzke-Kopp, L., Lynam, D., Reynolds, C., ... Liu, J. (2006). The ReactiveProactive Aggression Questionnaire: Differential Correlates of Reactive and Proactive Aggression in Adolescent Boys. Aggressive Behavior, 32(2), 159-171.

Scheier, M. F., Buss, A. H., \& Buss, M. (1978). Self-Report of Aggressiveness, and Aggression. Journal of Research in Personality, 12, 133-140.

Taylor, S. P. (1967). Aggressive behavior and physiological arousal as a function of provocation and the tendency to inhibit aggression. Journal of Personality, 35(2), 297-310.

Van Honk, J., \& Schutter, D. J. L. G. (2006). From affective valence to motivational direction: the frontal asymmetry of emotion revised. Psychological Science, 17(11), 963-5.

Veit, R., Lotze, M., Sewing, S., Missenhardt, H., Gaber, T., \& Birbaumer, N. (2010). Aberrant social and cerebral responding in a competitive reaction time paradigm in criminal psychopaths. Neurolmage, 49(4), 3365-72.

Vigil-Colet, A., Ruiz-Pamies, M., Anguiano-Carrasco, C., \& Lorenzo-Seva, U. (2012). The impact of social desirability on psychometric measures of aggression. Psicothema, 24(2), 310-5.

Walters, G. D. (2007). Measuring proactive and reactive criminal thinking with the PICTS: correlations with outcome expectancies and hostile attribution biases. Journal of Interpersonal Violence, 22(4), 371-85.

White, S. F., Brislin, S. J., Meffert, H., Sinclair, S., \& Blair, R. J. R. (2013). Callous-unemotional traits modulate the neural response associated with punishing another individual during social exchange: a preliminary investigation. Journal of Personality Disorders, 27(1), 99-112. 




\section{CHAPTER 8 General discussion}

"He allowed himself to be swayed by his conviction that human beings are not born once and for all on the day their mothers give birth to them, but that life obliges them over and over again to give birth to themselves."

— Gabriel García Márquez, Love in the Time of Cholera, 1985 

This thesis set out to investigate the neural correlates of impulse control. It was divided into three parts: Chapter 2 and 3 focused on the investigation of response inhibition networks; in chapter 4 and 5 the overlap of neural correlates between motor impulsivity and aggression was discussed and chapter 6 and 7 zoomed into the neural components underlying different forms of aggression. Functional Magnetic Resonance Imaging (fMRI) was employed to identify and localize neural networks involved in response inhibition, impulsivity, and aggression. Non-Invasive Brain Stimulation techniques, namely Transcranial Magnetic Brain Stimulation (TMS) and transcranial Direct Current Stimulation (tDCS), were applied to a) reveal mechanistic or causal relationship between the identified brain regions, response inhibition, and aggression and b) establish ways in which either inhibitory capacity or aggressive behaviour can be altered by manipulating the underlying neural mechanisms. In the following, the results of the empirical work presented in this thesis will be summarized and discussed. Resulting implications for future research in social neuroscience will be exemplified and potential applications of our findings in clinical contexts will be illustrated.

\section{Part I: The neural correlates of response inhibition}

\section{A common response inhibition network}

In a first step, we aimed to reveal the neural correlates of motor response inhibition. We hypothesized that different aspects of response inhibition correspond to different neural activation patterns, but also activate a global common inhibition network within the brain. The fMRI study " $A$ network approach to response inhibition: Dissociating functional connectivity of neural components involved in action restraint and action cancellation" (chapter 2) confirmed this hypothesis. Functional connectivity analysis revealed a common response inhibition network which was activated during response restraint and response cancellation comprising anterior insula cortex, inferior frontal cortex, pre-supplementary motor area, thalamus, and anterior cingulate cortex. Neural components specifically related to action restraint (i.e., right superior frontal gyrus, left middle frontal gyrus, and bilateral anterior cingulate cortex) and action cancellation (i.e., right middle frontal gyrus, posterior cingulate cortex, and parietal regions) were identified.

Inducing inhibitory deficits by means of TMS

For the follow-up study presented in chapter 3 ("The role of right prefrontal and medial cortex in response inhibition: Interfering with action restraint and action cancellation using transcranial magnetic brain stimulation") two nodes of the identified common inhibition network (right inferior frontal cortex / anterior insula, pre-supplementary motor area) and two specific neural components exclusively activated during action restraint (right superior frontal gyrus) versus action cancellation (right middle frontal gyrus) were targeted with functionally guided continuous Thetaburst Stimulation 


\section{CHAPTER 8}

(cTBS). In accordance with our hypothesis, interfering with the common inhibition network (by targeting the right inferior frontal cortex / anterior insula) impaired both aspects of response inhibition. Interfering with the right superior frontal cortex impaired specifically action restraint, but not action cancellation. No effects of cTBS over pre-supplementary motor area or right middle frontal gyrus on either aspect of response inhibition could be observed.

\section{Ecological validity}

Response inhibition - including action restraint and action cancellation - has been researched extensively in the field of cognitive neuroscience (Chambers, Garavan, \& Bellgrove, 2009; Swick, Ashley, \& Turken, 2011). Insights into the neural correlates of motor inhibition increase the understanding of the human motor system and advance knowledge on specific aspects of executive functioning and cognitive control. Therewith, it is possible to empirically assess numerous higher order concepts and pathologies that are related to deficits in cognitive control. However, when translating findings derived from cognitive neuroscience to the realm of social interaction (as done in the context of this thesis), one has to be aware of the fact that problems regarding ecological validity may arise. Everyday life requires forms of impulse control which are different from and / or more complex than simple motor response inhibition. So far, most response inhibition research and the simplifying paradigms it employs do not take that into account. In everyday life situations (avoiding a conflict, reacting calmly to a provocation), usually prior knowledge of which processes are to be inhibited in which way is available. Furthermore, rather conscious as opposed to automatic responses have to be inhibited. Response inhibition research should also focus on developing paradigms and neural models that are closer to realistic complex behaviours.

\section{Part II: The overlap of neural networks involved in motor impulsivity and aggression}

The neural overlap of motor impulsivity and aggression

In the second part of this thesis, the overlap of neural correlates involved in motor impulsivity and aggression was discussed. In chapter 4 ("Out of control: Evidence for anterior insula involvement in motor impulsivity and reactive aggression") we described our fMRI work revealing this overlap. In this study response inhibition and aggression were investigated on brain level, within the same participants, and - most importantly - assessed with behavioural in contrast to self-report measures. Our findings suggest common anterior insula involvement in motor impulsivity as well as reactive aggression and, thus, an overarching role of this region across different modalities of self-control. This finding enables an integrative perspective on insula function and a better integration of cognitive, social, and emotional factors into a comprehensive model of impulsivity. 


\section{Behavioural paradigms in social neuroscience}

Although the study took great effort to implement measures of motor impulsivity and behavioural aggression within an imaging environment (cf. Krämer, Jansma, Tempelmann, \& Münte, 2007), it has to be stressed that future research is needed to refine these measures. Especially for fMRI studies, it is crucial to work out task designs that can disentangle neural components involved in core versus peripheral cognitive processes (e.g. actual aggressive act versus perception, attention etc. required by the situation). It is of importance that paradigms enable the investigation of very specific contrasts. In the present case it is evident that the investigated contrast for the response inhibition task (based on false alarms in a go/no-go paradigm) did not only measure motor impulsivity, but might also have picked up on processes of error monitoring and behavioural adjustment. The Taylor Aggression Paradigm used in the study also provided us with limited samples of behaviour (e.g. participants hardly reacted aggressively to the non-provoking opponents, thus, proactive and reactive aggression could not directly be compared). Further refinement of fMRI paradigms in social neuroscience will enable a sharper capturing of the behavioural components of interest.

\section{An attempt to alter inhibitory capacity and aggressive behaviour by means of tDCS}

After revealing the overlap of motor impulsivity and aggression on neural level, we manipulated the excitability of the inferior frontal cortex - a region that we found to play a role in response inhibition and aggression - by means of bilateral tDCS ("No effects of bilateral tDCS over inferior frontal gyrus on response inhibition and aggression", chapter 5). We investigated, whether motor response inhibition and / or aggressive behaviour could be altered by this intervention. This approach was based on our previous work, but also on the cortical asymmetry theory of motivational direction (Eddie Harmon-Jones, 2004; van Honk \& Schutter, 2006) which states that approach motivation is mainly related to left prefrontal brain activity, while avoidance motivation is mainly related to right prefrontal activity. The concepts investigated in this thesis are associated with these two types of motivation; response inhibition is a behaviour related to avoidance motivation and aggression is seen to be a behaviour related to approach motivation (Carver \& Harmon-Jones, 2009). Based on this theory, we expected that enhancing the right inferior frontal gyrus (while simultaneously inhibiting the left) should increase the ability to inhibit motor responses and at the same time decrease aggressive behaviour, whereas enhancing the left inferior frontal gyrus (while simultaneously inhibiting the right) should have the exact opposite effect. We indeed revealed an inverse relationship between response inhibition and aggressive behaviour; the worse someone's ability to restrain a response, the more aggression he/she displayed. However, no effects of bilateral prefrontal tDCS on either response inhibition or aggression were observed. Therefore, this study could not provide evidence in support of the prefrontal cortical asymmetry model in the domain of response inhibition and aggression. The absence of tDCS effects might indicate that the methodologi- 


\section{CHAPTER 8}

cal approach of shifting cortical asymmetry by means of bilateral tDCS protocols failed, and/or that targeting inferior frontal cortex instead of the dorsolateral prefrontal cortex is not advisable.

\section{The hype-hope curve of tDCS}

TDCS is a relatively new method which originally attracted enormous attention as it promises cheap, fast, and comfortable applicability in all kinds of (clinical) settings. Recently, one senses some notion of disillusion in the community working with Direct Current Stimulation. Reviews even stated that tDCS would have little to no physiological effects (Horvath, Forte, \& Carter, 2014) and no effects on cognitive processes (Horvath, Forte, \& Carter, 2015). The mentioned reviews emphasized that tDCS studies published so far come along with various methodological weaknesses. Therefore, it is currently advisable to see the method as well as the current practice of its implementation with caution.

This phenomenon of quickly changing perception of methodologies in science was described as the 'Hype-Hope Cycle' (gartner.com): Triggered by technological invention, a method or topic reaches the 'peak of inflated expectations' relatively fast, after which the 'trough of disillusionment' follows. However, the curve does not stop there. There are still two more stages to follow; disillusion should trigger the 'slope of enlightenment' ultimately leading to 'the plateau of productivity'. These final stages are still to come in tDCS research. By now, we have to illuminate which physiological effects the method entails, how it is best applied, and for which processes and which participants / patients it is most effective. The 'hype' around tDCS seems to slowly fade away, but it is likely that at a later stage a more differentiated discussion on the method will evolve and more thorough knowledge on the working principles underlying it will be available. With tDCS, Non-Invasive Brain Stimulation research discovered a potentially powerful tool that still needs to be understood better.

\section{Part III: Understanding aggression on brain level}

The role, segregation, and lateralization of insular cortex in retaliation

In a third step, we focused on the neural correlates of aggressive behaviour. In chapter 6 , entitled "Left posterior insular activation during retaliation", parametric modulations of brain activity related to aggressive behaviour were analyzed in order to further investigate the role of the insular cortex in aggression. The insular cortex was repeatedly reported to be involved in aggression. Based on Craig's (2009) suggestion that emotions triggering immediate action tendencies and intense bodily feelings should be related to activity in the posterior insula, it was expected that aggression or retaliation related brain activity should also be located in the posterior part of the insular cortex. Our second hypothesis was that this activation should be left lateralized as the left prefrontal cortex has repeatedly been associated with approach motivation, anger 
processing, and aggression (Carver \& Harmon-Jones, 2009; Harmon-Jones \& Allen, 1998; Peterson, Shackman, \& Harmon-Jones, 2008). Our fMRI data indeed showed that the left posterior insula is modulated by the intensity of aggressive behaviour displayed during retaliation.

\section{New perspectives on insula function}

In the context of this thesis, insular activation has not only been demonstrated for aggression, but also for successful response inhibition (chapter 2 and 3) and motor impulsivity (chapter 4). This leads to the question in how far insular involvement is specific to any of its proposed functions. In fact, the insular cortex has been associated with numerous concepts that are seemingly contradictory such as interoception, mindfulness, self-awareness, motor inhibition, processing of positive emotions, processing of negative emotions (for review see Craig, 2009), aggression (Krämer et al., 2007), love (Cacioppo, Bianchi-Demicheli, Frum, Pfaus, \& Lewis, 2012), and others. In the context of cognitive control, the insular cortex has been described as a region modulating with stimulus saliency and urgency (Menon \& Uddin, 2010; Nelson et al., 2010; Sterzer \& Kleinschmidt, 2010). Maybe the puzzle around insula function could be solved, if functional brain research does no longer mainly focus on rather isolated single processes, but takes social and emotional contexts into account when explaining the functional roles of brain regions. For instance, Reynolds and Berridge (2008) demonstrated that varying emotional environments retune the function of neural populations. They showed that neurons in the nucleus accumbens of rats encode alternately for fear or pleasure depending on the environment the animals were exposed to (home-like, versus low stress, versus high stress). This discovery suggests that neural components might alter their functional involvement according to the social situation in which they are recruited. Other fields, such as immunology or epigenetics, already recognize the role of environmental and social factors in modifying biological processes (Cacioppo et al., 2000; Bird, 2007). The insular cortex might be highly relevant in different circumstances of intense emotions which require consequent behavioural responses. Most probably, it thereby adapts its function to whatever requirements have to be met. Further research, involving methodology reaching beyond hemodynamic neuroimaging techniques and taking into account varying environments, is needed to shed light on how the functional involvement of brain regions is modulated by social and emotional factors.

\section{Reducing aggression by enhancing the prefrontal cortex}

In the concluding study of this thesis ("Reducing proactive aggression through NonInvasive Brain Stimulation", chapter 7) we aimed to investigate whether aggressive behaviour can be altered by enhancing the right dorsolateral prefrontal cortex with tDCS. Thereby, based on the cortical asymmetry theory of motivational direction it was expected that an enhancement of this right prefrontal region should cause an increase 
in avoidance motivation and, thus, decrease aggression. Our work showed that specifically proactive aggression in males could be decreased by applying tDCS to the right prefrontal cortex.

It should be emphasized that we found tDCS to reduce specifically proactive aggression in males but not in females. Females have shown to be display less physical aggression than males (Archer, 2004; Bjorkqvist, 1994; Eagly \& Steffen, 1986; Lagerspetz, Bjorkqvist, \& Peltonen, 1988) and did so in our sample. The lack of effect of the stimulation in females might be caused by a floor effect considering that female students in our restricted sample displayed low aggression levels. We did not expect that brain stimulation would specifically decrease proactive, but not reactive, aggression. Proactive aggression refers to the instrumental use of aggression to obtain a reward or a prey (Anderson \& Bushman, 2002). Therefore, the motivation to approach seems central. The experimental manipulation in our study enhanced the right dorsolateral prefrontal cortex. This area is said to be responsible for emotional and cognitive processes generating avoidance motivation (Carver \& Harmon-Jones, 2009; HarmonJones, 2004). The assumption that the applied brain stimulation protocol enhanced avoidance and, thus, lowered approach motivation, fits with our finding that it reduced proactive aggression.

\section{Empirical evidence for the cortical asymmetry theory of motivational direction}

Although other stimulation parameters were employed and a different region within the right prefrontal cortex was targeted, these positive results are somehow contradicting our previous work (chapter 5). While enhancing the inferior frontal cortex by means of a bilateral tDCS protocol did not have effects on either response inhibition or aggression, enhancing the right dorsolateral prefrontal cortex with a unilateral protocol decreased aggressive behaviour in males. Both studies were based on preceding imaging work (chapter 4 and 6 ) and the cortical asymmetry theory of motivational direction. Our findings presented in chapter 7 are in accordance with the theoretical framework assigning avoidance motivation to the right hemisphere and approach motivation to the left hemisphere. However, the findings presented in chapter 5 fail to provide direct empirical evidence of this theory, bearing in mind all methodological problems which might have led to the null finding (see above). Based on the work in this thesis, it should be considered that the described theory might not always yield direct empirical evidence. Instead of merely looking at right and left prefrontal cortex, future research could investigate which precise parts of the prefrontal cortex can be associated with cortical asymmetry theories with respect to approach and avoidance motivation. Brain stimulation research will have to provide more empirical evidence, taking into account various regions within the prefrontal cortex, and various cognitive and emotional processes related to approach versus avoidance motivation, in order to reveal fine-grained inter-hemispheric differences. The human prefrontal cortex which is mostly assigned the function of cognitive control - seems to be relevant for 
countless aspects of behaviour (Miller \& Cohen, 2001; Miller, 2000). One might wonder if this complexity does not prevent a precise description of the region. After investigating the prefrontal cortex in the context of this thesis, I would carefully suggest that it is indeed involved in a wide range of human behaviours on cognitive and social level and that due to its complexity the decipherment of its specific functions and spatial segregation will keep neuroscientific research busy in the future.

\section{Current challenges in social neuroscience}

In the introduction to this thesis, it was stressed that successful research on complex behaviour has to be based on a multilevel approach (Cacioppo et al., 2000). This also holds true for neuroscience. If we want to advance our knowledge on brain function in a comprehensive way, neuroscience also needs to take into account the social and environmental factors that shape neural networks and modulate functional involvement of specific brain regions. Social neuroscience is the field that can and should address such questions, taking into account insights from other fields such as animal research, basic neuroscience, cognitive neuroscience, and the neuroscience of emotions. To accomplish this, it is crucial to understand and embrace methodological and technical advances and to establish multidisciplinary research networks. The following section will describe some of the current challenges in social neuroscience arising from the findings discussed in this thesis:

Social neuroscience can profit from insights derived from cognitive neuroscience. The starting point of this thesis was the investigation of a well-known concept in research on cognitive control and executive functioning. We first worked on the identification and localization of a global response inhibition network within the brain. This was a step to empirically approach higher order concepts and finally social interactions related to inhibitory processing. Social neuroscience might be subjected to critical voices doubting the empirical accessibility of complex social behaviours. These doubts are in part justified and it is therefore crucial for the field to ground its investigations in insights that are thoroughly established in other branches of neuroscience. For instance, theories established in other areas of neuroscience can be a source of inspiration for the development of research questions in social neuroscience. An example relevant for this thesis was the cortical asymmetry theory on motivational direction. Research in social neuroscience on the other hand can provide further empirical evidence for these already established theories, but also give new differential perspectives on the contexts in which these theories are or are not applicable.

Social neuroscience should use multiple and high standard methodologies. The thesis at hand investigated response inhibition and aggression using a multi-method approach. Functional imaging was used to measure neural correlates while NonInvasive Brain Stimulation was used to manipulate these correlates and observe subsequent changes in behaviour. Combining both methodologies and exploiting their 
specific strengths led to a comprehensive picture of the neural mechanisms underlying impulse control. Besides $\mathrm{fMRI}$ and brain stimulation, also electroencephalography, electromyography, single cell recordings, animal models, and other promising methods should be used in social neuroscience. It is advisable that social neuroscience follows a multi-method approach in order to provide empirical evidence on the concepts at investigation from multiple perspectives.

As outlined above, the development of well-controlled behavioural paradigms that can pick up on very fine-grained aspects of social behaviour is crucial for social neuroscience. Task development is always challenging, but even more so, when social interactions (complex behavioural patterns comprising various sub-components) are to be investigated. The correct balance between internal and external validity has to be established. Paradigms have to be well controlled, but still mirror real life behaviour as close as possible.

Finally, social neuroscience should contribute to other areas of neuroscience by examining the influence of social and environmental factors on brain function. This might open up possibilities to explain variability in the functions assigned to specific brain areas. In this thesis, this approach was discussed in the context of insula involvement in retaliation (chapter 6 and see above). Thereby, neuroscience can follow in the footstep of genetics, immunology, and other fields in investigating how biological processes influence social and environmental outcome, but even more how social and environmental factors shape their underlying biological mechanisms.

Aside from the mentioned points, social neuroscience should confront the problems currently present in experimental research: Results should be reproducible, transparency should be key when reporting findings, experiments should have sufficient statistical power, and data should be made available for the community upon publication. Furthermore, it would benefit the field, if the publication bias towards significant and consistent results would be decreased and exploratory research would not be reported as confirmatory.

\section{Future outlook - clinical applications}

The results presented in this thesis (in particular chapter 7) are a first step towards potential clinical applications. It has to be emphasized that our findings have to be subjected to well-controlled thorough replication, before they can be generalized. Furthermore, the findings presented in the context of this thesis are based on group level analyses, and do not necessarily hold true on a single subject level. Before NonInvasive Brain Stimulation protocols that have an effect on single subject level can be established, more research needs to be done. Impulsivity is a construct not only relevant for cognitive and differential psychology, but also the field of psychopathology. Disorders including attention deficit hyperactivity disorder, obsessive compulsive disorder, eating disorders, personality disorders of cluster B (e.g. borderline, antisocial), 
and various forms of addiction are related to deficits in impulse control. If Non-Invasive Brain Stimulation can alter impulse control by enhancing activity within the prefrontal cortex, it provides us with a powerful tool, which can potentially lead to therapeutic interventions. Both, TMS and tDCS have been applied successfully in clinical contexts such as for instance in the treatment of depression, stroke, tinnitus, migraine and others (Brunoni et al., 2012; George, Taylor, \& Short, 2013; Nitsche et al., 2008; Wassermann \& Lisanby, 2001). However, so far studies on brain stimulation interventions adressing deficits in impulse control are scarce. The work outlined in the context of this thesis encourages such studies. Results derived from healthy samples regarding inhibitory networks in the brain, prefrontal asymmetry, impulse control, and aggression have to be transferred to clinical contexts. Investigating more extreme populations in which impulsivity issues are more prevalent than in healthy populations, will enable an even better understanding of the concepts at hand and, furthermore, smoothen the way for directly brain based interventions that go without the wide spread side effects of psychiatric medication. Impulsivity related disorders pose enormous stress to patients and their environment. They challenge therapeutic settings and our societal health care systems. Developing further interventions in this context, based on NonInvasive Brain Stimulation techniques such as TMS or tDCS, will be a major task for future research.

\section{Conclusion or the omitted chapter}

As outlined in the introduction to this thesis, when the Bristish author Anthony Burgess published his novel 'A Clockwork Orange' in the United States, he was forced to omit the 21st chapter. In the original final chapter the main character (named Alex) reflects upon his violent behaviour, repents his actions, and makes a resolution to adapt his future behaviour to societal norms. This ending, however, did not seem chatchpenny enough to either the oversea's publisher or Stanley Kubrick's cinematic adaption of the material. Only in 1994 literary scholars voted for the rehabilitation of the omitted ending.

It is probably the dark side of aggression, on which note the shortened version of the novel ends, that causes omnipresent fascination for violence. However, to me it seems that the less selling question of whether or not aggressive behaviour can be modified by external or internal means and, thus, if aggressive individuals can be reintegrated into society, is ultimately the more spectacular question to answer. Burgess' 21st chapter might be the most relevant one for the 21st century.

During the last decade, describing the 'criminal brain' was of common interest in forensic circles. Adrian Raine, a well-known forensic psychologist, recently published a book entitled 'The Anatomy of Evil: The biological Roots of Crime' (2014). In his book, he describes in a very accessible way, how neuroscientists set out to identify the deviances in neural mechanisms within the brains of violent offenders. Deficiencies in brain 
function are outlined alongside genetic patterns and other biological deviations. Raine mentions that, when looking at these biological substrates of aggression, a dangerous trap to fall into is to assume that violent offenders are just 'natural born killers'. If aggressive behaviour is merely rooted in biological factors and brain deficiency, this could suggest that offenders can a) not be held responsible for their actions and $b$ ) that if offenders just have 'murderous minds' there is no way to modulate their behaviour in the future. However, from a scientific perspective this trap should be avoided.

Although not conducted in forensic samples, the work presented in this thesis can contribute to this discussion. We showed that aggressive behaviour can be elicited in healthy university students, and that, therefore, most probably all of us have the potential to behave aggressively (as well as the potential to behave prosocially) preprogrammed in our brains. This means that plenty of factors have to come together in order to make a 'criminal brain' actually commit a crime. The mechanism at work here is neither one-dimensional nor causal. Although neuroscientific evidence is (and can be) used in court, it can never lead to the assumption of immunity of the offender as long as no severe alterations in the offender's state of consciousness during the offense can be proven. Some of us might live with what Raine calls a 'murderous mind', but due to environmental factors and advanced impulse control, never act on it. Therefore, also the idea that fMRI could be used as a tool to predict future aggressive behaviour or to plead for guilty / not guilty in court has to be seen with caution. Having a certain biological disposition cannot be equaled to behaving on it.

Furthermore, we provide evidence, as do other studies, that aggressive behaviour can be manipulated directly on biological or brain level. We showed that the enhancement of prefrontal cortex by means of Non-Invasive Brain Stimulation can decrease aggressive behaviour under specific circumstances. Taking into account the enormous plasticity of the brain, it will be of interest to further explore brain based treatment options in a forensic context, which can support established forms of cognitive and behavioural interventions. Taken together, all these available interventions can target aggression on various levels and lead to changes in the behaviour of highly aggressive individuals.

Imagine that Alex from 'A Clockwork Orange' would have undergone Non-Invasive Brain Stimulation instead of the 'Ludovico technique'. Probably he would have suffered less and would have emerged from the story not as a broken man but as a responsible individual, capable of changing his behaviour. 
Aron, A. R. (2011). From reactive to proactive and selective control: developing a richer model for stopping inappropriate responses. Biological Psychiatry, 69(12), e55-68.

Archer, J. (2004). Sex Differences in Aggression in Real-World Settings: A Meta-Analytic Review. Review of General Psychology, 8(4), 291-322.

Bird, A. (2007). Perceptions of epigenetics. Nature, 447(7143), 396-8.

Bjorkqvist, K. (1994). Sex differences in physical, verbal, and indirect aggression: A review of recent research. Sex Roles, 30(3-4), 177-188.

Brunoni, A. R., Nitsche, M. A., Bolognini, N., Bikson, M., Wagner, T., Merabet, L., ... Fregni, F. (2012). Clinical research with transcranial direct current stimulation (tDCS): challenges and future directions. Brain Stimulation, 5(3), 175-95.

Cacioppo, J. T., Berntson, G. G., Sheridan, J. F., \& McClintock, M. K. (2000). Multilevel integrative analyses of human behavior: social neuroscience and the complementing nature of social and biological approaches. Psychological Bulletin, 126(6), 829-43.

Cacioppo, S., Bianchi-Demicheli, F., Frum, C., Pfaus, J. G., \& Lewis, J. W. (2012). The common neural bases between sexual desire and love: a multilevel kernel density fMRI analysis. The Journal of Sexual Medicine, 9(4), 1048-54.

Carver, C. S., \& Harmon-Jones, E. (2009). Anger is an approach-related affect: evidence and implications. Psychological Bulletin, 135(2), 183-204.

Chambers, C. D., Garavan, H., \& Bellgrove, M. A. (2009). Insights into the neural basis of response inhibition from cognitive and clinical neuroscience. Neuroscience and Biobehavioral Reviews, 33(5), 631-46.

Craig, A. D. B. (2009). How do you feel - now? The anterior insula and human awareness. Nature Reviews Neuroscience, 10(1), 59-70.

Gartner, hype-hope curve, retrieved from http://www.gartner.com/technology/ research/methodologies/hype-cycle.jsp, 3.02.2015.

George, M. S., Taylor, J. J., \& Short, E. B. (2013). The expanding evidence base for rTMS treatment of depression. Current Opinion in Psychiatry, 26(1), 13-8.

Eagly, A. H., \& Steffen, V. J. (1986). Gender and aggressive behavior: a meta-analytic review of the social psychological literature. Psychological Bulletin, 100(3), 309-30.

Harmon-Jones, E. (2004). Contributions from research on anger and cognitive dissonance to understanding the motivational functions of asymmetrical frontal brain activity. Biological Psychology, 67(1-2), 51-76.

Harmon-Jones, E., \& Allen, J. J. (1998). Anger and frontal brain activity: EEG asymmetry consistent with approach motivation despite negative affective valence. Journal of Personality and Social Psychology, 74(5), 1310-6.

Horvath, J. C., Forte, J. D., \& Carter, O. (2014). Evidence that transcranial direct current stimulation (tDCS) Generates little-to-no reliable neurophysiologic effect beyond MEP amplitude modulation in healthy Human subjects: A systematic review. Neuropsychologia, 66, 213-236.

Horvath, J. C., Forte, J. D., \& Carter, O. (2015). Quantitative Review Finds No Evidence of Cognitive Effects in Healthy Populations from Single-Session Transcranial Direct Current Stimulation (tDCS). Brain Stimulation, in print, doi:10.1016/j.brs.2015.01.400.

Krämer, U. M., Jansma, H., Tempelmann, C., \& Münte, T. F. (2007). Tit-for-tat: the neural basis of reactive aggression. Neurolmage, 38(1), 203-11.

Lagerspetz, K. M. J., Bjorkqvist, K., \& Peltonen, T. (1988). Is Indirect Aggression Typicai of Females?? Gender Differences in Aggressiveness in, 14, 403-414.

Menon, V., \& Uddin, L. Q. (2010). Saliency, switching, attention and control: a network model of insula function. Brain Structure \& Function, 214(5-6), 655-67.

Miller, E. K. (2000). The prefrontal cortex and cognitive control. Nature Reviews Neuroscience, 1(1), 59-65.

Miller, E. K., \& Cohen, J. D. (2001). An integrative theory of prefrontal cortex function. Annual Review of Neuroscience, 24, 167-202.

Nelson, S. M., Dosenbach, N. U. F., Cohen, A. L., Wheeler, M. E., Schlaggar, B. L., \& Petersen, S. E. (2010). Role of the anterior insula in task-level control and focal attention. Brain Structure \& Function, 214(56), 669-80. 


\section{CHAPTER 8}

Nitsche, M. a, Cohen, L. G., Wassermann, E. M., Priori, A., Lang, N., Antal, A., ... Pascual-Leone, A. (2008). Transcranial direct current stimulation: State of the art 2008. Brain Stimulation, 1(3), 206-23.

Peterson, C. K., Shackman, A. J., \& Harmon-Jones, E. (2008). The role of asymmetrical frontal cortical activity in aggression. Psychophysiology, 45(1), 86-92.

Raine, A. (2014). The anatomy of evil: The biological roots of crime. NY: Vintage.

Reynolds, S. M., \& Berridge, K. C. (2008). Emotional environments retune the valence of appetitive versus fearful functions in nucleus accumbens. Nature Neuroscience, 11(4), 423-5.

Sterzer, P., \& Kleinschmidt, A. (2010). Anterior insula activations in perceptual paradigms: often observed but barely understood. Brain Structure \& Function, 214(5-6), 611-22.

Swick, D., Ashley, V., \& Turken, U. (2011). Are the neural correlates of stopping and not going identical? Quantitative meta-analysis of two response inhibition tasks. Neurolmage, 56(3), 1655-65.

Van Honk, J., \& Schutter, D. J. L. G. (2006). From affective valence to motivational direction: the frontal asymmetry of emotion revised. Psychological Science, 17(11), 963-5.

Wassermann, E. M., \& Lisanby, S. H. (2001). Therapeutic application of repetitive transcranial magnetic stimulation: a review. Clinical Neurophysiology, 112(8), 1367-77. 


Summary 

The empirical work presented in this thesis investigated brain networks involved in inhibition and aggression. Functional imaging and Non-Invasive Brain Stimulation were combined in order to measure and manipulate the neural correlates of impulse control.

The fMRI study "A network approach to response inhibition: Dissociating functional connectivity of neural components involved in action restraint and action cancellation" (chapter 2) revealed a global response inhibition network involved in various forms of response inhibition. Additionally, specific neural components related exclusively to either response restraint or response cancellation were detected. Subsequently, this global network as well as the specific neural components were targeted with cTBS ("The role of right prefrontal and medial cortex in response inhibition: Interfering with action restraint and action cancellation using transcranial magnetic brain stimulation", chapter 3) and global as well as specific inhibitory deficits could be induced.

Chapter 4 ("Out of control: Evidence for anterior insula involvement in motor impulsivity and reactive aggression") focuses on the investigation of neural overlap between motor impulsivity and behavioural aggression employing $\mathrm{fMRI}$ during a response inhibition and a controlled behavioural aggression paradigm. When manipulating the excitability of one overlapping region in inferior frontal cortex by means of bilateral tDCS ("No effects of bilateral tDCS over inferior frontal gyrus on response inhibition and aggression", chapter 5), effects on neither response inhibition, nor aggressive behaviour could be demonstrated.

Finally, the role of the insular cortex in aggression was investigated ("Left posterior insular activation during retaliation", chapter 6). By analyzing parametric modulations of brain activity related to aggressive behaviour it was revealed that the posterior part of the left insular cortex is involved in retaliation; its possible specific role is discussed in detail. In the concluding study ("Reducing proactive aggression through NonInvasive Brain Stimulation", chapter 7) it was demonstrated that specifically proactive aggression can be decreased in males by enhancing the right dorsolateral prefrontal cortex by means of tDCS.

All results are summarized and discussed in chapter 8 ("General discussion"). Implications for future research, resulting challenges in social neuroscience, and potential clinical applications of the results outlined in this thesis are illustrated. 

Valorization 



\section{RELEVANCE}

The empirical work presented in this thesis entitled 'A network approach to response inhibition and aggression: Combining functional imaging and Non-Invasive Brain Stimulation in the study of impulse control' is relevant for a variety of societal questions.

Each year, over 180.000 registered 'violent crimes' (including murder, manslaughter, rape, sexual coercion, robberies, dangerous and serious bodily injury, and slight bodily injury with intent) are registered e.g. by German authorities (Polizeiliche Kriminalstatistik PKS / Bundeskriminalamt BKA, 2013). In the United States, violent crimes amount to over one million per year (Uniform Crime Report UCR / Federal Bureau of Investigation $\mathrm{FBI}, 2013$ ). The prevalence of aggressive behaviour is one of the main challenges our modern civilization has to face. It leads to the experience of unsafety in all of us and poses enormous costs to our social systems. Therefore, it is crucial to provide the public, forensic professionals, and workers dealing with aggressive individuals (in schools, hospitals, and the social sector) with scientific evidence on 1) possible etiological models and 2) potential interventions able to decrease impulsivity and aggression. The empirical work presented in this thesis does both: Employing functional imaging it is providing evidence on the neural correlates of successful as well as failed impulse control and aggression. This leads to a better understanding of the underlying neural mechanisms. Furthermore, by employing Non-Invasive Brain Stimulation it provides evidence on how impulse control can be manipulated and thus opens up possibilities towards directly brain-based interventions.

Besides its general societal relevance, impulsivity and aggression are crucial components of various clinical disorders such as attention deficit hyperactivity and conduct disorder, obsessive compulsive disorders, certain expressions of schizophrenia, borderline and antisocial personality disorder, as well as psychopathy. Our health care systems are responsible for treating children, adolescents, adult patients, and criminal offenders displaying these pathologies. Such treatment poses enormous costs. It is necessary to increase the quality of life of the concerned individuals and their environment as well as to ensure their (re)integration into society. To constantly better understand these pathologies and the mechanisms underlying their inhibitory deficits is the foundation of advanced treatment options. Thereby, deficits in impulse control have to be understood on behavioural and biological level. Furthermore, by not only identifying but also finding ways to manipulate the underlying mechanisms on behavioural and biological level, the corner stone for clinical interventions is set. The empirical work presented in this thesis contributes to a better understanding of neural mechanisms underlying impulsivity and aggression, and furthermore makes a first step towards manipulating these mechanisms in order to ultimately decrease impulsivity and aggression. 


\section{TARGET GROUP}

The results presented in this thesis are of interest to several occupational groups. First and foremost, professions dealing with highly impulsive or aggressive individuals in a clinical, forensic, or developmental context can profit from our work. Therefore, we published in scientific journals relevant to these professions and presented our results on various national and international conferences.

We believe that the results presented in this thesis are also of relevance to the general public and, thus, gave interviews resulting in several newspaper articles.

\section{ACTIVITIES / PRODUCTS}

Ultimately, the results presented in our thesis could lead to the development of direct brain-based interventions in order to improve impulse control and decrease aggression. However, in order to reach this goal many steps have to be taken before. First, our results have to be replicated in healthy and clinical samples. Second, they have to be validated on single subject level.

\section{INNOVATION}

Our results are innovative, because they directly address the question in how far response inhibition and aggression are related on brain level. In our work we use a multimethod approach combining functional imaging in order to identify relevant neural networks and various forms of Non-Invasive Brain Stimulation to modify impulse control by manipulating the underlying neural mechanisms. This approach enables an overarching look on response inhibition and aggression.

\section{SCHEDULE AND IMPLEMENTATION}

Future plans include translating our findings on response inhibition to more naturally valid forms of inhibition. Furthermore, we plan to replicate the findings presented in chapter 7 and to test the (in part) successful brain stimulation protocol in a clinical context including highly impulsive individuals. Depending on the outcome, we will work on further combining a potential brain stimulation intervention with inhibition training and develop new treatment options in order to improve impulse control and decrease aggressive behaviour. 


Acknowledgements 



\section{色不異空}

One single person could never do 'science'. Scientific research is driven by teamwork, by cooperation, by sharing happiness as well as frustration, by giving and seeking advice, by drinking coffee together and by helping each other out. I could not have done any of this on my own and want to sincerely thank all the people who helped me on my way. Some of them I want to mention in person:

Alex, I still wonder why you agreed that I could apply for the PhD position you and Jill were offering in the fall of 2011; aside from a great interest in the topic I had neither a neuroscientific background nor any other skills that seemed relevant at the time. Well, I am happy that you did! You opened up many new possibilities for me. I could never have developed more than I did by working within your group. I want to thank you for your supervision, your fairness, your focus, your scientific wisdom, and for always seriously trying to help me solving each and every problem arising on the way. I learned (and will learn) a lot from you! I could not imagine a better (as we call it in German) 'Doktor-Vater' than you have been to me. Thank you.

I was very lucky, as I did not just have a 'Doktor-Vater' but practically 'DoktorEltern'. Teresa, what I learned from you goes far beyond how to handle a TMS coil. You encouraged me, you motivated me, you always stayed positive despite my (often numerous) doubts. Together with Alex you always gave me the feeling that you believed in me. This was more important to me than you might think. Without you, Teresa, I could not have done it. I appreciate your attitude, you sense of humor, and how you always figure out what really matters. I can consider myself lucky to have had so many great conversations with you on professional and personal matters. Thank you for being present (and I mean it!).

Jill, thank you for being a mentor and adviser throughout my PhD. I admire you for your genuine passion for science, your focus, and more than anything your calmness. You are a role model to me. Once you told me that at one point you understood that talking faster and/or louder does not mean that what is said is more meaningful and that you ever since just follow your own rhythm. I am still working on this (and many other things), but I want to say that you inspire me. I hope that we will work together in the future.

Arnoud, it was an honor to profit from your wisdom and your incredibly sharp mind. You challenged me and always made things better. Thank you for being my copromotor and thank you for your great support!

Suzanne, it is always a pleasure working with you. It is enriching to share time and ideas with you. I wish you and your young family the very very best.

I would not have managed my job and - more importantly - I would not have been so happy during the past years, if it weren't for the research group I was part of. I want to thank every member of the NIBS-group for making it a great group with a very constructive work attitude and at the same time so social. Alex, Teresa, Nina, Christianne, 
Tom, Felix, Rosanne, Tim, Helen, Tahnee, Dennis, Lukas, Geraldine, and everybody else: You are the best! Thanks also to the angel of the TMS-Labs - Jeannette. Jeannette, without your tremendous help my experiments would never have worked; thanks also for always listening. Helen, although we do not know each other for so long, we share a lot; thanks for your company, your encouragement, and for being my paranymph. Nina, we were office mates, colleagues, coaches, and had conversations about so many topics - thanks to you and your horses for helping and for the green in my life. Felix, I want you to know that I deeply respect your work attitude, your knowledge, and your cooperative spirit. Tom, thanks for always sharing your honest opinion on life, science, career, and doing a PhD, whenever I asked for it.

For me, the $C N$ department is a great work environment. This is due to the dedication of all leadership personnel and each and every colleague (including former colleagues). I will not mention everyone by name (because I could never find a just way to do so), but all of you contribute to the climate we have. I want to sincerely thank you for everything you directly or indirectly did for me, for your help, and for all the wonderful conversations, evenings, and trips I had with you! Thanks to each of you.

I also want to thank my collegues from CPS. I always enjoyed working together with you, thinking about PhD issues for the graduate school, and having many nice conversations and dinner dates. Special thanks to Carolien, Jessica, and Kai.

Thanks to all collaboration partners, reviewers, mentors, and everyone who shared knowledge on the way. I thank all members of my reading committee and my corona. Bernadette, Ulrike, Chris, Vincent, Carolien thanks for the time and effort you put into the evaluation of my thesis. I was lucky enough to encounter a very open minded and cooperative climate in science, which does not hold true for every young researcher. This was due to the marvelous attitude of my supervision team and the fellow scientists I met on my way. I very much hope that this is the attitude we can further promote in the context of scientific research.

Thanks to my roommates. Nina, Valerie, Aline, Roberta, and Shruti, all of you were wonderful companions. I enjoyed working in one office with every one of you. Thank you for sharing our interests. Lars, thank you for every conversation and every moment of silence together! I appreciate it very much.

To Christl, Riny, and Eva: There are no words - what would I have done without you?!?!?! Thank you.

Banditos: You brew our coffee, but actually it's more than just coffee - you make us alive. Thanks for every smile, for every chat, for every cup, for feeding me. Thanks for your great work.

Thanks to the team of Brain Innovation, especially to its 'prophet' Armin and to Judith. I also want to thank the Scannexus team for their help with my fMRI work.

I thank all students that were involved in the projects presented in this thesis. Amongst others: The fabulous Martijn \& Martijn, the smart Oana, the talented Clara, the indispensable Anke, and all the awesome 2.5 kids. It is a privilege working with you 
and teaching you. Your energy and joy makes research fun. Without such dedicated students, universities could never work. I wish you all the best for your doubtlessly bright futures.

Rarely mentioned, but most crucial: To all participants. Thank you for taking part in our studies. Without volunteers, cognitive and social neuroscience would not advance at all. I most appreciate the time and effort our participants put into the many many experimental sessions which were conducted in order to complete this thesis. Thank you!

To the Looiersgracht Gang: Thanks to king Richard and the entire royal family; meeting you enriched our life in Maastricht. Thanks to Joyce for being Joyce!!! Tom and Uno - it's good to have you with us. Christoph, I enjoy living next to you. Thank you for the company as neighbor, colleague, and - well - great friend. I admire how you live your life and you inspire me. Nora, you are the sunshine of our courtyard, you are always interested, always questioning; I learn a lot from you and I love spending time with you.

To Rik and Cath. It is difficult to put into words what I want to say to you. If we would have never met you, we would have missed you without knowing it. You made Maastricht feel home. Without you, we would have lived here without knowing anybody or anything, without getting outside and playing, without your great company and most of all - without family. You mean the world to me. Rik, you do not like to hear 'Thank you'. So, no 'thanks' to you, although you would deserve one. You should know that I admire you for how you are and what you do. You are crazy and a true artist, an artist of life. Catharina, you became one of my best friends. I owe you a lot; you know what I mean. Thank you for always being there for me. In all the good and bad moments. For helping. For caring. For sharing time, thoughts, tea, and food. For being a true friend. And for standing behind me on D-day.

I want to thank 'my ladies'. Leonie, Martina, Corinna, Ilona, and Uschi, it is an honor to know you and to have worked, laughed, doubted, and discussed with you. Getting to know you widened my horizon tremendously. I very much hope that our contact survives through all upcoming times, wherever we will go and whatever we will do.

Thank you Angela Guzzardi - without our conversations things would be very different. You were an enormous help and a person to look up to.

Thanks to all teachers and friends from the TZ. You changed many things in my life for the better. It is unique to know you and to learn from and with you. Each day we spend together in Hamburg and Lünzen is a treasure to me.

More than anyone I have to thank my many loyal friends who stayed with me from school times (Elisabeth, Silke, Juliane) to university (Julia, Nancy, Lena, Oscar, Christine) all over the world (Emmanuel, Pablo, Mariangela). There have been many situations in my life that I would not have mastered without your presence and many wonderful moments I would have never enjoyed as much without you. Thank you! 
To family Kigawa: You gave me a second home. You have always been there for me. There are no words that could thank you enough for your support!

I want to thank my family in Augsburg, Eppstein, Berlin, and elsewhere. Being part of a huge family has shaped me more than anything else. Every one of you contributed to what I am today. Although I cannot mention all of you by name, you know how much you mean to me and how you have contributed.

Dear Ulli and Konni, one could not wish for better godfathers. You were the influence in my life, which made me look behind the 'Tellerrand'. You showed me different ways of life and different areas one could be interested in. My favorite books from childhood are the ones I got from you. By now I have developed many many interests and these interests make me happy every day.

Meine liebe Haje, Du warst meine erste richtige Freundin und Gefährtin. Du hast meine Kindheit und Jugend immer mit Deiner Lebenspraxis und Deiner Wärme begleitet. Du, Deine Pfannkuchen, die Gurkenstraße, der Gartenzwerg und Ödipussi bedeuten mir so viel! Ich kann Dir nicht genug dankbar sein.

Liebe Klotzoma, Du bist mir eine wunderbare Großmutter. Von Dir habe ich Stil und Eleganz gelernt. Von Opa mein Interesse an der Wissenschaft. Ihr habt mich sehr geprägt und ich bin Euch unendlich dankbar dafür.

Winfried und Pamela, Ihr habt mich mit (im wahrsten Sinne des Wortes) offenen Armen begrüßt. Dafür danke ich Euch sehr! In den letzten Jahren standet Ihr mir immer mit Rat und Tat zur Seite. Dank Euch fühle ich mich in der Schillerallee zu Hause. Ich freue mich, bald Eure Schwiegertochter werden zu dürfen. Danke auch an Martin mit Familie und die phänomenale Marianne!

Meinen Schwestern: Schwestern zu haben ist einmalig; anders als Freundschaft, Familie oder Partnerschaft. Ich bin glücklich darüber, dass es Euch gibt und ich habe Euch beide sehr lieb. Johanna, ich kann mich noch gut daran erinnern, wie sehr wir uns gefreut haben, als Du auf die Welt gekommen bist. Schön, dass es Dich gibt. Ich danke Dir für unseren Kontakt und ich hoffe, dass ich für Dich (und Du für mich), dass wir füreinander, Zeit unseres Lebens da sein werden. Resi, es fällt mir schwer die richtigen Worte zu finden. Ich bewundere Dich von ganzem Herzen, den Weg den Du zusammen mit Marie gehst, Deine Menschenkenntnis und Deine Liebe zum Leben. Ich kann mich sehr sehr glücklich schätzen, dass Du meine Schwester und Ratgeberin bist. Danke. Vielen Dank.

Auch wenn all die oben genannten maßgeblich dazu beigetragen haben, dass ich zu der geworden bin, die ich heute bin, gibt es doch zwei Menschen, denen eine besondere Rolle zukommt: Liebe Eltern, ich danke Euch dafür, dass Ihr uns aufgezogen habt und uns Kinder immer an erste Stelle gestellt habt. Ihr habt mir ohne Wenn und Aber den größten Schatz in meinem Leben ermöglicht: meine Ausbildung. Für all die Mühen, die Euch das vom Windelwechseln bis zur Finanzierung meines Studiums gekostet haben muss, danke ich Euch von Herzen. Papa, Du warst ein Vater, der da war. Du hast mit mir gesungen, bist mit mir ins Schwimmbad gefahren und hast mit mir gespielt. Ich 
danke Dir fürs Vater-Sein. Inzwischen bist Du vom Vater zum Ratgeber geworden und ich hoffe, dass wir auch in Zukunft alles miteinander besprechen können. Mama, meine komplette Grundausstattung habe ich von Dir mitbekommen. Durch Dich habe ich Konsequenz, Durchhaltevermögen und Disziplin gelernt. Ohne diese Eigenschaften hätte ich meine Promotion niemals so geschafft. Ich danke Dir für all die Zeit, die Du in mich investiert hast. Mama und Papa, Ihr ward als Eltern ein gutes Team. Ihr habt den Weg dafür geebnet, dass ich zu einer sehr glücklichen und zufriedenen Frau geworden bin. Ich wünsche Euch von Herzen alles Gute für die Zukunft und dass Ihr das, was ab von Arbeit und Kindererziehung für Euch kommen wird, in vollen Zügen genießen werdet!

Thomas, viele haben vor mir versucht, an dieser Stelle die richtigen Worte zu finden. Das Glück, dass wir uns begegnet sind, ist nicht in Worte zu fassen. Ich liebe Dich. Ich schätze und bewundere Dich. Durch Deine Liebe habe ich den Mut gefunden, im Leben das zu tun, was mich interessiert. Du hast auf so viele Arten dazu beigetragen, dass ich meine Dissertation umsetzen konnte. Theoretisch, praktisch und emotional. Du bist der beste Partner, den man sich vorstellen kann, und (wie Du schon oft und von vielen gehört hast) einer der besten und aufrichtigsten Menschen, die auf diesem Planeten herumlaufen. Jeder Tag mit Dir ist ein Vergnügen. Die letzten Jahre waren für uns erfüllt von wunderbaren Gesprächen, kreativen Ideen, großen Reisen, aufregenden Neuerungen und spannenden Herausforderungen. Ich hoffe, dass es für uns so weitergeht. In guten wie in schlechten Zeiten. Ich danke Dir - für alles. 

Curriculum vitae 

Franziska Dambacher was born on July 13th 1986 in Augsburg, Germany. She completed her high school education at the Theodor-Heuss-Gymnasium Nördlingen in 2005 and subsequently studied Psychology and Theology (minor) at the University of Trier, Germany. During her studies she was student research assistant at the Center for High Giftedness at the University of Trier under Prof. Dr. Francis Preckl and at the Institut for Psychology of Religion, Bad Kreuznach, under Prof. Dr. Sebastian Murken. In 2008/09 she received a full scholarship of the Japanese Student Service Organization (JASSO) and spent one year studying philosophy at Sophia University, Tokyo, Japan. In September 2011 she started working as a PhD student at the Faculty of Psychology and Neuroscience at Maastricht University, in both the Department of Cognitive Neuroscience and the Department of Clinical Psychological Science. In the context of this joined project she investigated the neural correlates of response inhibition and aggression employing functional imaging and Non-Invasive Brain Stimulation and was supervised by Prof. Dr. Alexander T. Sack, Dr. Teresa Schuhmann, Dr. Jill Lobbestael, and Prof. Dr. Arnoud Arntz. In 2012 she received the Young Investigators Award of the Society of Research on Aggression (ISRA) and in 2014 a scholarship to attend the summer school on Social and Cognitive Neuroscience (SISSA) in Trieste, Italy. In 2015 she completed her three-year degree as systemic counsellor and family coach (DGSF) at the Systemic Institute Euregio (SIE), Eschweiler, Germany. From September 2015, Franziska will be working as a post-doctoral fellow at the Vision, Awareness, and Cognition Group of Prof. Dr. Alexander T. Sack at Maastricht University. 


\section{PUBLICATIONS}

Dambacher, F., Schuhmann, T., Lobbestael, J., Arnzt, A., Brugman, S. \& Sack A. T. (2015). Reducing proactive aggression through Non-Invasive Brain Stimulation. Social Cognitive and Affective Neuroscience, in print.

Brugman, S., Lobbestael, J., Arntz, A., Cima, M., Schuhmann, T., Dambacher, F., \& Sack, A. T. (2014). Identifying cognitive predictors of reactive and proactive aggression. Aggressive Behavior, in print.

Dambacher, F., Sack, A.T., Lobbestael, J., Arnzt, A., Brugman, S. \& Schuhmann, T. (2014). Out of control: Evidence for anterior insula involvement in motor impulsivity and reactive aggression. Social Cognitive and Affective Neuroscience, epub ahead of print.

Dambacher, F., Sack, A. T., Lobbestael, J., Arntz, A., Brugman, S., \& Schuhmann, T. (2014). The Role of Right Prefrontal and Medial Cortex in Response Inhibition: Interfering with Action Restraint and Action Cancellation Using Transcranial Magnetic Brain Stimulation. Journal of Cognitive Neuroscience, 26(8), 1775-84.

Dambacher, F., Sack, A. T., Lobbestael, J., Arntz, A., Brugman, S., \& Schuhmann, T. (2014). A network approach to response inhibition: dissociating functional connectivity of neural components involved in action restraint and action cancellation. The European journal of neuroscience, 39(5), 821-831

Murken, S. \& Dambacher, F. (2014). Entry 'Dissociation'. In K. von Stuckrad \& R. A. Segal (eds.), Vocabulary for the Study of Religion. Leiden (NL) \& Boston: Brill Academic Publishers.

Murken, S. \& Dambacher, F. (2014). Neue religiöse Bewegungen, Sekten oder Midnerheiten? Anmerkungen zur öffentlichen Wahrnehmung kleiner religioeser Gruppierungen. In E. Franke (ed.), Religiöse Minderheiten und gesellschaftlicher Wandel. Wiesbaden: Harrassowitz.

Dambacher, F., Murken, S. \& Lehmann, K. (2011). Quantitative Zugänge zu religiösen Phänomenen: Statistische Analyseverfahren und ihr Potential für die Religionswissenschaften. In S. Kurth \& K. Lehmann (eds.), Lehrbuch Religionen erforschen. Wiesbaden: VS Verlag für Sozialwissenschaften. 
Murken, S. \& Dambacher, F. (2010). Religion, Freizeit und Tourismus. Ich bin dann mal weg: Religionspsychologische Überlegungen zur gegenwärtigen Popularität von Pilgerreisen. Psychologie in Österreich, 30(1), 6-12.

\section{PUBLICATIONS SUBMITTED, AND IN PREPARATION}

Dambacher, F., Schuhmann, T., Lobbestael, J., Arnzt, A., Brugman, S. \& Sack A. T. (2015). The role of the insular cortex in retaliation, submitted.

Dambacher, F., Schuhmann, T., Lobbestael, J., Arnzt, A., Brugman, S. \& Sack A. T. (2015). No effects of bilateral tDCS over inferior frontal gyrus on response inhibition and aggression, submitted.

Dambacher, F., Duecker, F., Schuhmann, T., Adams, J., Sack A. T.(2015). When foresight is easier than hindsight: The neural correlates of the anti-cue paradigm, in preparation.

\section{CONFERENCE PRESENTATIONS}

2014, Annual meeting, Society for Neuroscience, Washington D.C., USA

Poster presentation "Shifting the balance: Interfering with motor inhibition and reactive aggression by inducing fronto-cortical asymmetry using transcranial direct current stimulation (tDCS)"

2014, Annual meeting, Organization for Human Brain Mapping, Hamburg, Germany Poster presentation "Out of control: Evidence for anterior insula involvement in motor impulsivity and reactive aggression"

2013, Annual meeting, Society for Neuroscience, San Diego, USA

Poster presentation "The role of right prefrontal cortex in response inhibition: Interfering with action restraint and action cancellation using transcranial magnetic stimulation (TMS)"

2013, European Winterschool on Antisocial Behavior, Seeon, Germany

Talk "The role of inhibitory control in aggression: A project employing functional Magnetic Imaging (fMRI) and Transcranial Magnetic Stimulation (TMS)"

2012, Annual meeting, International Society for Research on Aggression, Luxembourg Poster presentation "The role of inhibitory control in aggression - an fMRI study" 
